\title{
NOXious oxidative stress : from head toe too and back
}

Citation for published version (APA):

Kleikers, P. W. M. (2014). NOXious oxidative stress : from head toe too and back. [Doctoral Thesis, Maastricht University]. Maastricht University. https://doi.org/10.26481/dis.20141208pk

Document status and date:

Published: 01/01/2014

DOI:

10.26481/dis.20141208pk

Document Version:

Publisher's PDF, also known as Version of record

\section{Please check the document version of this publication:}

- A submitted manuscript is the version of the article upon submission and before peer-review. There can be important differences between the submitted version and the official published version of record.

People interested in the research are advised to contact the author for the final version of the publication, or visit the DOI to the publisher's website.

- The final author version and the galley proof are versions of the publication after peer review.

- The final published version features the final layout of the paper including the volume, issue and page numbers.

Link to publication

\footnotetext{
General rights rights.

- You may freely distribute the URL identifying the publication in the public portal. please follow below link for the End User Agreement:

www.umlib.nl/taverne-license

Take down policy

If you believe that this document breaches copyright please contact us at:

repository@maastrichtuniversity.nl

providing details and we will investigate your claim.
}

Copyright and moral rights for the publications made accessible in the public portal are retained by the authors and/or other copyright owners and it is a condition of accessing publications that users recognise and abide by the legal requirements associated with these

- Users may download and print one copy of any publication from the public portal for the purpose of private study or research.

- You may not further distribute the material or use it for any profit-making activity or commercial gain

If the publication is distributed under the terms of Article $25 \mathrm{fa}$ of the Dutch Copyright Act, indicated by the "Taverne" license above, 
NOXious oxidative stress:

from head to toe and back 
NOXious oxidative stress: from head to toe and back

Thesis, Maastricht University, Maastricht, The Netherlands ISBN: 9789461088086

(c) P.W.M. Kleikers, 2014

Design and layout: P.W.M. Kleikers and W.D.J. Lafeber

Printed by: Gildeprint 


\section{NOXious oxidative stress:}

from head to toe and back

\section{PROEFSCHRIFT}

Ter verkrijging van de graad van doctor aan de Universiteit Maastricht, op gezag van Rector Magnificus, Prof. dr. L.L.G. Soete, volgens het besluit van het College van Decanen, openbaar te verdedigen op maandag 8 december om 14.00 uur

door

Pamela Wilhelmus Maria Kleikers

Geboren op 21 september 1985 te Heerlen 


\section{Promotores}

Prof. dr. H.H.H.W. Schmidt

Prof. dr. H.W.M. Steinbusch

\section{Co-promotor}

Dr. B.J.A. Janssen

\section{Beoordelingscommissie}

Prof. dr. H. Struijker-Boudier (voorzitter)

Prof. dr. A. Daiber (Universität Mainz)

Prof. dr. B. Kramer

Prof. dr. K.H.K. Krause (Geneva University)

Prof. dr. R.J. van Oostenbrugge

Financial support by the Dutch Heart Foundation for the publication of this thesis is gratefully acknowledged. 


\section{Contents}

$\begin{array}{lll}\text { Chapter } 1 & \text { Introduction to oxidative stress in cardiovascular disease } & 7\end{array}$

$\begin{array}{lll}\text { Chapter } 2 & \text { NADPH oxidases as a source of oxidative stress and } 17\end{array}$ molecular target in ischemia/reperfusion injury

Chapter 3 Identification of endothelial cells as a major vascular source of NADPH oxidase 4-derived ROS causing blood brain barrier disruption, inflammation and neuronal damage in ischemic stroke

Chapter 4 A preclinical randomized, controlled trial approach for pharmacological target validation: The case against NOX2 in stroke

Chapter 5 To the toe: organ specificity and different isoforms

Part 1 'Mouse matter(s)'

Part 2 The heart: NOX4 does not play a major role in myocardial infarction or ischemia-reperfusion

Part 3 The hindlimb: neither NOX4, nor NOX1, 2 or 5 contribute to chronic ischemia in the femoral artery ligation model'

Chapter 6 Back to the brain: the role of NOX5 in stroke and hypertension

Chapter 7 General discussion

Chapter 8 Summary

Chapter 9 Samenvatting

$\begin{array}{lll}\text { Chapter } 10 \quad \text { Valorisation addendum } & 217\end{array}$

Appendices Dankwoord

List of publications

About the author 
Chapter 1

Introduction to oxidative stress in cardiovascular disease 
Cardiovascular diseases are increasing in human society and they are the number one cause of mortality worldwide, responsible for 17 million deaths (around $30 \%$ of all) ${ }^{1}$. Ischemic heart disease and stroke are the major contributors and their mortality rates increased dramatically over the past decade (Fig 1 ). In the past decades, all cardiovascular disease have emerged as an epidemic problem and they are projected to keep growing in the coming years ${ }^{2}$.

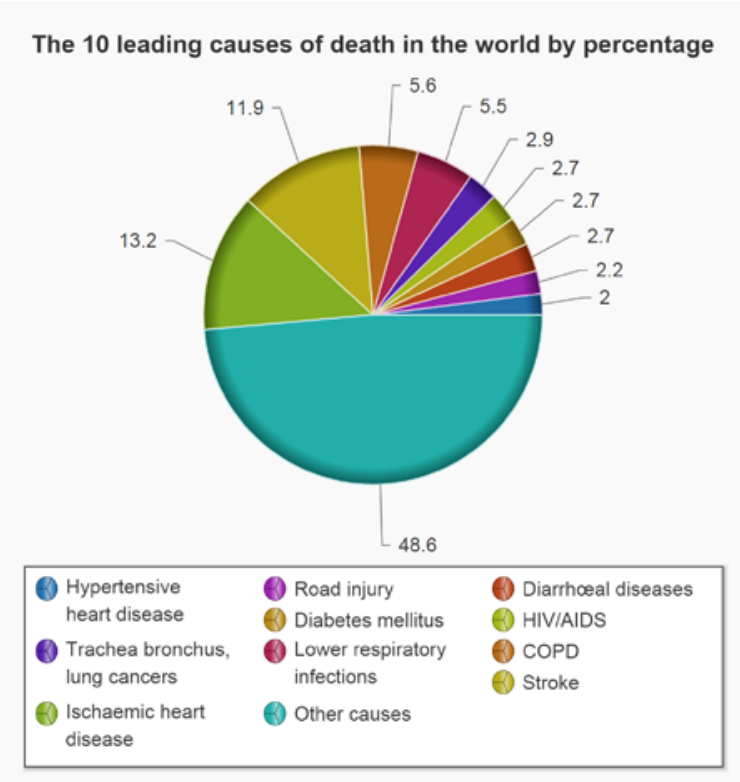

A

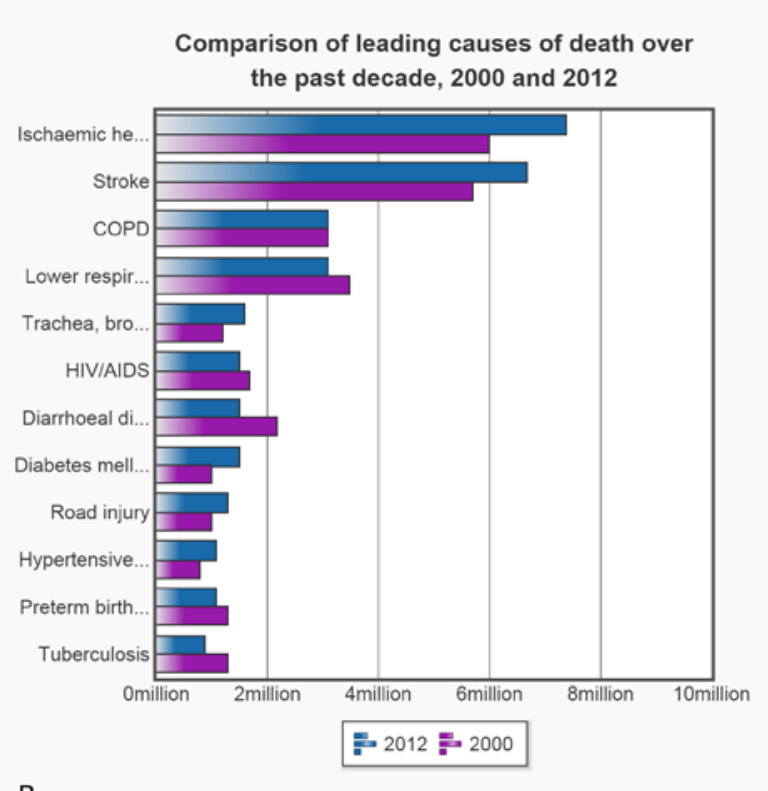

B

Figure 1. Mortality rates worldwide A) Top ten leading causes of death worldwide showing the large contribution of ischemic heart disease and stroke. B) Deaths due to ischemic heart disease and stroke have increased in the past decade [from WHO].

One major pathomechanism that is relevant for all (ischemic) cardiovascular diseases is oxidative stress. Reactive oxygen species (ROS) have been implicated in many different disease models and organ systems: cancer ${ }^{3}$, different inflammatory diseases ${ }^{4,5}$ and cardiovascular conditions ${ }^{6,7}$. In addition, also risk factors for cardiovascular disease, such as hypertension ${ }^{8}$, metabolic syndrome ${ }^{9}$ and diabetes ${ }^{10,11}$ have been found to be associated with increased levels of oxidative stress. Endothelial dysfunction seems to be one of the major consequences of ROS formation that accounts for the damage of oxidative stress ${ }^{12}$. 


\section{Oxidative stress}

In the cardiovascular system, a tightly regulated balance exists between pro- and antioxidant systems. The term 'oxygen paradox' ${ }^{13}$ already implies that oxygen can have both positive and negative effects on the cardiovascular system. Oxygen is not only needed as a direct nutrient but is also important for normal cellular processes. The formation of ROS on the other hand can be harmful to almost all cells and tissues. A disbalance in the oxidative status, either by an increase in pro-oxidants or a decrease in anti-oxidants, or both, leads to an overload of detrimental ROS. This disbalance is called 'oxidative stress' 6 6,14,15.

In a state of oxidative stress, ROS can harm cells directly by oxidizing DNA ${ }^{16}$, opening the mitochondrial permeability transition pore ${ }^{6}$, lipid peroxidation ${ }^{17}$ or activation of matrix metalloproteinases ${ }^{16}$. Indirectly, an increased inflammation or the formation of even more cytotoxic substances (by reacting with other molecules such as NO or free iron) contribute to damage ${ }^{6,17}$.

\section{NADPH oxidases}

Several endogenous sources of ROS exist, such as xanthine oxidase ${ }^{18,19}$, the mitochondrial electron transport chain ${ }^{20,21}$ and uncoupled nitric oxide synthase ${ }^{22}$. All these producers of reactive oxygen species do so as a by-product of their main metabolism or as a result of dysfunction. One ROS-source stands out, as they are the only enzymes with the sole function of producing ROS: the NADPH oxidases ${ }^{23}$. These six -or seven transmembrane enzymes, of which 7 isoforms exist (Fig. 2), have emerged as an important source of reactive oxygen species in the cardiovascular system ${ }^{23-25}$. They are multi-complex proteins which all have two heme groups, an NADPH and an FAD binding site ${ }^{26}$. Enzymatically, they catalyse the one electron transfer from NADPH to molecular oxygen, producing ROS ${ }^{23,27,28}$. Within the cardiovascular system, NOX1, NOX2, NOX4 and NOX5 can be found. As is explained in Table 1 and Fig. 1 of Chapter 1 of this thesis ${ }^{29}$ the isoforms differ in their tissue and subcellular distribution, their activation, the subunits and the sort of ROS produced ${ }^{30}$. NOX4 is unique in that it is constitutively active and produces mainly hydrogen peroxide, NOX5 is exceptional because it is not present in the rodent genome and it can be activated by calcium. 

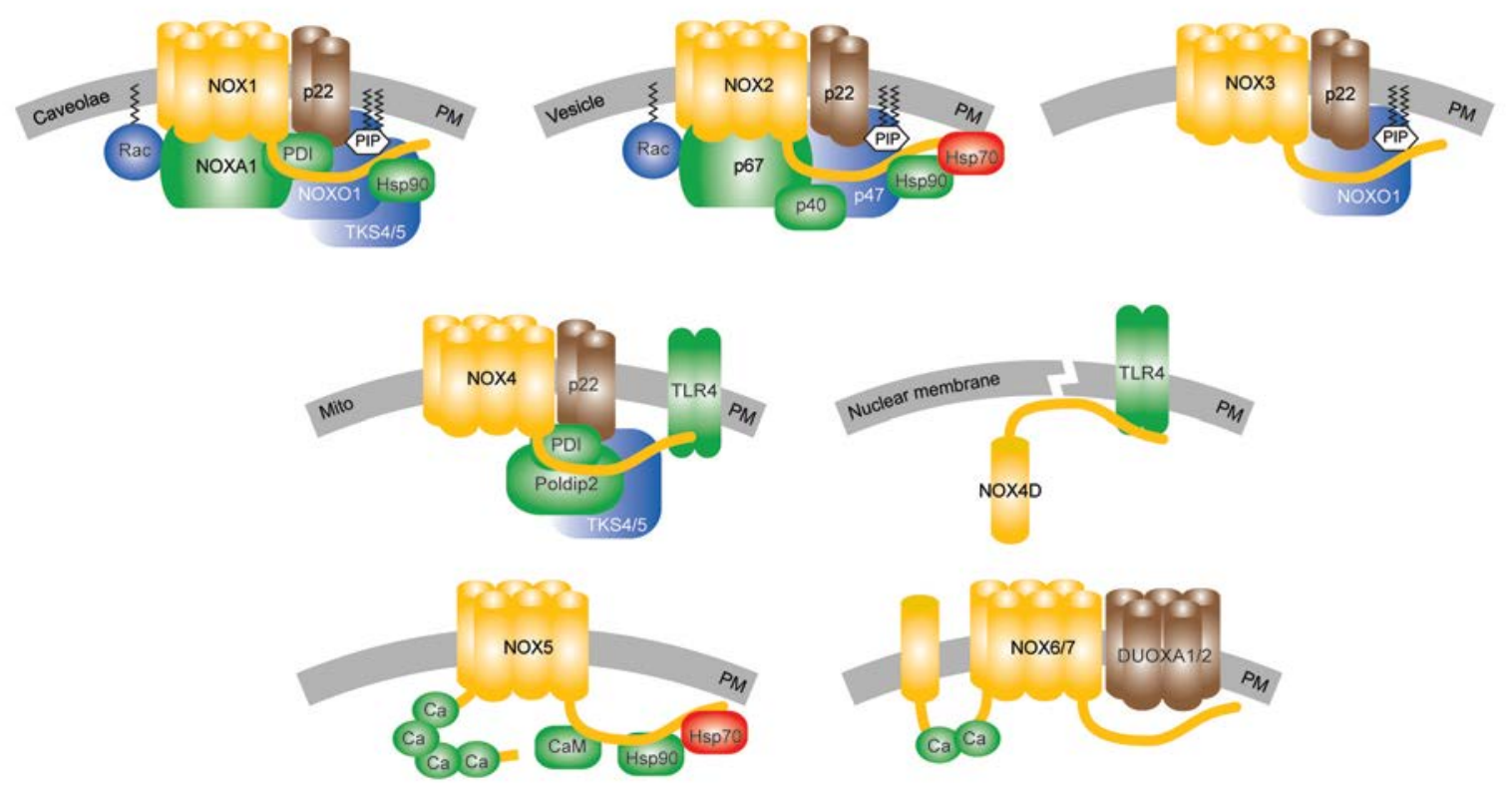

Figure 2. Overview of the seven NOX isoforms (yellow) with their different subcellular localisations (in gray) and their different binding partners. NOX1, 3 and 4 form complexes with the membrane protein p22 ${ }^{\text {phox }}$. In addition, NOX organisers (NOXO1, the small GTPase Rac, p47 $7^{\text {phox }}$ and tyrosine kinase substrate with 4/5 SH3 domains (TSK4/5), depicted in blue) and NOX activators (NOXA1, $p 67^{\text {phox }}$ and $p 40^{\text {phox }}$ ) are needed for active complex formation. Additional proteins such as heat shock proteins (HSP) have been recently found to play a role in NOX activation (HSP90) or destabilisation (HSP70, in red). NOX4 is constitutively active but can be activated by protein disulphide isomerase (PDI) and protein polymerase (DNA-directed) delta-interacting protein 2 (poldip 2). NOX4 can also be regulated by toll-like receptor 4 (TLR4). A soluble splice variant of NOX4, called NOX4D can be found in the nuclear membrane and misses five of the six transmembrane domains. NOX5 has intracellular calcium binding domains and can thus be activated by calcium. Calmodullin (CaM) can enhance the calcium sensitivity. NOX6/7 were formerly called DUOX1/2, they need the activators DUOXA1/2 (respectively) for functioning and can also be activated by calcium. [from ${ }^{31}$ ]

\section{The ROS paradox}

Although it is clear that oxidative stress and ROS are involved in many cardiovascular diseases, antioxidants used to reduce the amount of oxidative stress have not been shown to work.

A recent meta-analysis including 50 studies with almost 300.000 patients did not show any benefit of using antioxidants for prevention of cardiovascular disease ${ }^{32}$. 
Lots of smaller clinical trials have been conducted showing no benefit or even harmful effects of antioxidants, as will be discussed in Chapter 1 in more detail.

Apparently, there is not only an oxygen paradox but also a ROS paradox: in small amounts at the right place in the cell/tissue, ROS are needed for normal physiologic functions and cellular processes such as growth and differentiation ${ }^{33,34}$. But if the amount of ROS is too overwhelming or if the ROS are formed in the wrong subcellular space, damage can easily occur.

\section{Ischemia-reperfusion injury}

Both the oxygen paradox and the ROS paradox are present in the pathology of ischemia-reperfusion injury. During blockade of an artery, blood and oxygen supply subsides, causing damage to the tissue ${ }^{35,36}$. This first phase is the ischemic phase. Since oxygen is necessary for tissue survival, reperfusion, either spontaneously or via an intervention, has to occur. During this second phase, the overwhelming amount of oxygen results in the formation of ROS, which can make the ischemic damage even worse ${ }^{17}$. In a third phase, the post-reperfusion phase, tissue adaptation and remodelling occurs, in which small amounts of ROS are needed for cell proliferation and differentiation. Angiogenesis is one such example where ROS are needed in the post-reperfusion phase, or with chronic ischemia ${ }^{37,38}$. NADPH oxidases have been shown to be involved in ischemia-reperfusion injury in different organs and during the different phases. In Chapter 2 of this thesis this role of NOX in ischemia-reperfusion injury is reviewed.

\section{Ischemic stroke}

Stroke is one of the major diseases where ischemia-reperfusion injury accounts for a huge burden in terms of mortality and morbidity. NADPH oxidase 4 has been shown to be a key player in experimental stroke, with NOX4 KO mice showing smaller infarct sizes, better neurological outcome and a better survival after middle cerebral artery occlusion.

What is not known, is which NOX4-expressing vascular cell type is responsible for the detrimental ROS formation. Chapter 3 describes the role of different NOX4expressing cell types in experimental stroke. 


\section{Quality issues in experimental stroke research}

Experimental stroke research has been at a roadblock for the last decades: over 1000 preclinical drug candidates for treating stroke, failed to ultimately reach the clinics ${ }^{39,40}$. Study design and quality issues in preclinical animal studies ${ }^{41,42}$ as well as insufficient power of these studies ${ }^{43}$ or publication bias ${ }^{44}$ may account for this poor clinical translational success. For NOX1 and 2, the role in experimental stroke was shown by several research groups but conflicting results are described in Kleinschnitz et al. ${ }^{45}$ Taking into account the possible problems of translational failure, lack of power and publication bias, in Chapter 4, a systematic review and preclinical blinded trial to finally clarify the role of NOX1/2 in ischemic stroke, are described.

\section{Organ specificity and isoforms}

While the role of NADPH oxidases in ischemic stroke has been extensively studied, their role in other forms of ischemia-reperfusion injury is less clear yet. Since the different isoforms have different cellular and subcellular localisation, cell and even organ specific effects of oxidative stress must be taken into account.

The oxygen and ROS paradox already implicate that different isoforms might have divergent roles in different organs. Also, there might be a difference between ischemia-reperfusion injury and chronic ischemic conditions. In Chapter 5 of this thesis, the role of the different vascular NOX isoforms are tested in both ischemiareperfusion and chronic ischemia of the heart and chronic hindlimb ischemia representing a model of angiogenesis.

\section{NOX5, the overlooked vascular NADPH oxidase}

Although the pathogenesis and concepts of ROS and oxidative stress are becoming more and more clearer and animal studies show promising results, the translation to the human clinical situation is not that straightforward.

In Chapter 6 of this thesis, we try to get a closer link between our animal experiments and human disease addressing both hypertension and stroke. 
None of the vascular NADPH oxidases present in rodents has been found to have a role in hypertension. NOX5, a gene not naturally present in the genome of the rodents has recently been described to be related to human hypertension ${ }^{46}$. Using a humanised NOX5 $\mathrm{KI}$ mouse model, the role of NOX5 in elevating blood pressure and increasing stroke gender-dependently, is described in Chapter 6.

\section{Scientific research questions}

Taken together, the following research questions will be addressed subsequently in this thesis:

1. Regarding the detrimental role of NOX4 in stroke, which vascular cell type expressing NOX4 can be held responsible?

2. Can quality or power issues explain the disagreement on the role of NOX1 and 2 in preclinical stroke research?

3. Does NOX4 play a role in ischemia-reperfusion injury in other organs than the brain?

4. Can the role of NOX in stroke be translated into the clinic?

\section{References}

1. World Health Organisation. Global status report on noncommunicable diseases 2010. 1-176 (2011).

2. Mathers, C. D. \& Loncar, D. Projections of global mortality and burden of disease from 2002 to 2030. PLoS Med. 3, e442 (2006).

3. Matés, J. M., Segura, J. A., Alonso, F. J. \& Márquez, J. Oxidative stress in apoptosis and cancer: an update. Arch. Toxicol. 86, 1649-1665 (2012).

4. Zuo, L., Otenbaker, N. P., Rose, B. A. \& Salisbury, K. S. Molecular mechanisms of reactive oxygen species-related pulmonary inflammation and asthma. Mol. Immunol. 56, 57-63 (2013).

5. Afonso, V., Champy, R., Mitrovic, D., Collin, P. \& Lomri, A. Reactive oxygen species and superoxide dismutases: role in joint diseases. Joint Bone Spine 74, 324-329 (2007). 
6. Elahi, M. M., Kong, Y. X. \& Matata, B. M. Oxidative stress as a mediator of cardiovascular disease. Oxid Med Cell Longev 2, 259-269 (2009).

7. Forstermann, U. Oxidative stress in vascular disease: causes, defense mechanisms and potential therapies. Nat Clin Pract Cardiovasc Med 5, 338349 (2008).

8. Harrison, D. G., Gongora, M. C., Guzik, T. J. \& Widder, J. Oxidative stress and hypertension. J Am Soc Hypertens 1, 30-44 (2007).

9. Otani, H. Oxidative stress as pathogenesis of cardiovascular risk associated with metabolic syndrome. Antioxid Redox Signal 15, 1911-1926 (2011).

10. Baynes, J. W. \& Thorpe, S. R. Role of oxidative stress in diabetic complications: a new perspective on an old paradigm. Diabetes 48, 1-9 (1999).

11. Lipinski, B. Pathophysiology of oxidative stress in diabetes mellitus. J. Diabetes Complicat. 15, 203-210 (2001).

12. Cai, H. \& Harrison, D. G. Endothelial dysfunction in cardiovascular diseases: the role of oxidant stress. Circ Res 87, 840-844 (2000).

13. Yellon, D. M. \& Hausenloy, D. J. Myocardial reperfusion injury. N. Engl. J. Med. 357, 1121-1135 (2007).

14. Valko, M. et al. Free radicals and antioxidants in normal physiological functions and human disease. Int. J. Biochem. Cell Biol. 39, 44-84 (2007).

15. Papaharalambus, C. A. \& Griendling, K. K. Basic mechanisms of oxidative stress and reactive oxygen species in cardiovascular injury. Trends Cardiovasc. Med. 17, 48-54 (2007).

16. Griendling, K. K. Oxidative stress and cardiovascular injury: Part I: basic mechanisms and in vivo monitoring of ROS. Circulation 108, 1912-1916 (2003).

17. Eltzschig, H. K. \& Collard, C. D. Vascular ischaemia and reperfusion injury. $\mathrm{Br}$ Med Bull 70, 71-86 (2004).

18. Berry, C. E. \& Hare, J. M. Xanthine oxidoreductase and cardiovascular disease: molecular mechanisms and pathophysiological implications. $J$. Physiol. (Lond.) 555, 589-606 (2004).

19. Harrison, R. Structure and function of xanthine oxidoreductase: where are we now? Free Radic Biol Med 33, 774-797 (2002). 
20. Di Lisa, F. \& Bernardi, P. Mitochondria and ischemia-reperfusion injury of the heart: fixing a hole. Cardiovasc Res 70, 191-199 (2006).

21. Gao, L., Laude, K. \& Cai, H. Mitochondrial pathophysiology, reactive oxygen species, and cardiovascular diseases. Vet Clin North Am Small Anim Pract 38, 137-55- vi (2008).

22. Otani, H. The role of nitric oxide in myocardial repair and remodeling. Antioxid Redox Signal 11, 1913-1928 (2009).

23. Cave, A. C. et al. NADPH oxidases in cardiovascular health and disease. Antioxid Redox Signal 8, 691-728 (2006).

24. Lassegue, B. \& Griendling, K. K. NADPH oxidases: functions and pathologies in the vasculature. Arterioscler Thromb Vasc Biol 30, 653-661 (2010).

25. Lambeth, J. D. NOX enzymes and the biology of reactive oxygen. Nat Rev Immunol 4, 181-189 (2004).

26. Rivera, J., Sobey, C. G., Walduck, A. K. \& Drummond, G. R. Nox isoforms in vascular pathophysiology: insights from transgenic and knockout mouse models. Redox Rep 15, 50-63 (2010).

27. Griendling, K. K., Sorescu, D. \& Ushio-Fukai, M. NAD(P)H oxidase: role in cardiovascular biology and disease. Circ Res 86, 494-501 (2000).

28. Geiszt, M. NADPH oxidases: new kids on the block. Cardiovasc Res 71, 289299 (2006).

29. Kleikers, P. W. M. et al. NADPH oxidases as a source of oxidative stress and molecular target in ischemia/reperfusion injury. J. Mol. Med. 90, 1391-1406 (2012).

30. Lassegue, B. \& Clempus, R. E. Vascular NAD(P)H oxidases: specific features, expression, and regulation. Am. J. Physiol. Regul. Integr. Comp. Physiol. 285, R277-97 (2003).

31. Altenhöfer, S., Radermacher, K. A., Kleikers, P., Wingler, K. \& Schmidt, H. H. Evolution of NADPH oxidase inhibitors: Selectivity and mechanisms for target engagement. Antioxid Redox Signal (2014). doi:10.1089/ars.2013.5814

32. Myung, S.-K. et al. Efficacy of vitamin and antioxidant supplements in prevention of cardiovascular disease: systematic review and meta-analysis of randomised controlled trials. BMJ 346, f10 (2013). 
33. Lambeth, J. D. Nox enzymes, ROS, and chronic disease: an example of antagonistic pleiotropy. Free Radic Biol Med 43, 332-347 (2007).

34. Dröge, W. Free radicals in the physiological control of cell function. Physiol. Rev. 82, 47-95 (2002).

35. Maxwell, S. R. \& Lip, G. Y. Reperfusion injury: a review of the pathophysiology, clinical manifestations and therapeutic options. Int J Cardiol 58, 95-117 (1997).

36. Gourdin, M. J., Bree, B. \& De Kock, M. The impact of ischaemia-reperfusion on the blood vessel. Eur J Anaesthesiol 26, 537-547 (2009).

37. Maulik, N. Redox regulation of vascular angiogenesis. Antioxid Redox Signal 4, 783-784 (2002).

38. Maulik, N. \& Das, D. K. Redox signaling in vascular angiogenesis. Free Radic Biol Med 33, 1047-1060 (2002).

39. Radermacher, K. A. et al. The 1027th target candidate in stroke: Will NADPH oxidase hold up? Exp Transl Stroke Med 4, 11 (2012).

40. Radermacher, K. A. et al. Neuroprotection after stroke by targeting NOX4 as a source of oxidative stress. Antioxidants and Redox Signaling 18, 1418-1427 (2013).

41. Macleod, M. R. et al. Good laboratory practice: preventing introduction of bias at the bench. Stroke 40, e50-2 (2009).

42. Braeuninger, S. \& Kleinschnitz, C. Rodent models of focal cerebral ischemia: procedural pitfalls and translational problems. Exp Transl Stroke Med 1, 8 (2009).

43. Button, K. S. et al. Power failure: why small sample size undermines the reliability of neuroscience. Nat. Rev. Neurosci. 14, 365-376 (2013).

44. Couzin-Frankel, J. When mice mislead. Science 342, 922-3- 925 (2013).

45. Kleinschnitz, C. et al. Post-stroke inhibition of induced NADPH oxidase type 4 prevents oxidative stress and neurodegeneration. PLOS Biol. 8, (2010).

46. $\mathrm{Yu}, \mathrm{P}$. et al. Unique role of NADPH oxidase 5 in oxidative stress in human renal proximal tubule cells. Redox Biol 2, 570-579 (2014). 


\section{Chapter 2}

NADPH oxidases as a source of oxidative stress and molecular target in ischemia/reperfusion injury

Kleikers et al (2012) Journal of Molecular Medicine, volume 90: 1391-1 


\begin{abstract}
Ischemia-reperfusion-injury (IRI) is crucial in the pathology of major cardiovascular diseases such as stroke and myocardial infarction. Paradoxically, both the lack of oxygen during ischemia and the replenishment of oxygen during reperfusion can cause tissue injury. Clinical outcome is also determined by a third, post-reperfusion phase characterized by tissue remodelling and adaptation. Increased levels of reactive oxygen species (ROS) have been suggested to be key players in all three phases. As a second paradox, ROS seem to play a double-edged role in IRI, with both detrimental and beneficial effects. These Janus-faced effects of ROS may be linked to the different sources of ROS or to the different types of ROS that exist and may also depend on the phase of IRI. With respect to therapeutic implications, an untargeted application of antioxidants may not differentiate between detrimental and beneficial ROS, which might explain why this approach is clinically ineffective in lowering cardiovascular mortality. Under some conditions antioxidants even appear to be harmful. In this review we discuss recent breakthroughs regarding a more targeted and promising approach to therapeutically modulate ROS in IRI. We will focus on NADPH oxidases and their catalytic subunits, NOX, as they represent the only known enzyme family with the sole function to produce ROS. Similar to ROS, NADPH oxidases may play a dual role as different NOX isoforms may mediate detrimental or protective processes. Unravelling the precise sequence of events, i.e. determining which role the individual NOX isoforms play in the various phases of IRI, may provide the crucial molecular and mechanistic understanding to finally effectively target oxidative stress.
\end{abstract}




\section{Introduction}

Stroke and myocardial infarction are two major causes of death and disability in Western countries. Both are caused by organ ischemia followed by varying degrees of reperfusion. Reactive oxygen species (ROS) are thought to be the key players in this ischemia-reperfusion injury (IRI) ${ }^{1,2}$. Consequently, treatment of IRI should be directed to these key players. The untargeted use of antioxidants, however, failed to show any clinical benefits after stroke ${ }^{3,4}$ or myocardial infarction ${ }^{5,6}$. Indeed, specifically targeting the pathological source of ROS may provide a better therapeutic approach. Among such potential ROS sources, NADPH oxidases are the only known enzymes with the sole function of producing ROS. In the cardiovascular system, NAPDH oxidases account for a major part of the ROS formed, not only during IRI but also under physiologic conditions ${ }^{7}$. Here, we review the pathophysiology of IRI, focusing on NADPH oxidases as new potential targets for therapeutic interventions.

\section{Ischemia-reperfusion injury: two paradoxes}

During ischemia or the subsequent reperfusion damage can occur in cells or tissues, which often is irreversible. This damage is referred to as ischemia reperfusion injury (IRI) ${ }^{1,8}$. IRI can be divided into three phases. During the first, ischemic phase, interruption of the blood-flow to an organ causes a (temporary) lack of oxygen and nutrients ${ }^{1,2,8,9}$. The generation of ATP via oxidative phosphorylation is disturbed during ischemia, causing cells to alter their metabolism. The reduced availability of ATP limits the activity of the ATP-dependent $\mathrm{Na}^{+} / \mathrm{K}^{+}$pump ${ }^{1,2}$, which constrains the outflow of calcium via the $\mathrm{Na}^{+} / \mathrm{Ca}^{2+}$ exchanger ${ }^{8}$. This results in calcium overload, causing structural disorganisation as well as apoptotic and necrotic death of cells 2,8,9. In addition, chemokines and adhesion molecules promote a pro-inflammatory state ${ }^{1,2,10}$. Moreover, the altered cellular metabolism leads to an accumulation of precursors of oxidative phosphorylation, and it causes damage to or conformational changes of enzymes such as xanthine oxidase. These latter processes are not directly detrimental to cells, but they become important in the next phase of IRI by increasing ROS generation ${ }^{1,8,9}$.

The second phase of IRI constitutes the re-establishment of blood flow and thus the re-introduction of oxygen. 
Although oxygen is needed for survival, it can also be detrimental because it can be converted to ROS such as hydrogen peroxide $\left(\mathrm{H}_{2} \mathrm{O}_{2}\right)$ and superoxide $\left(\mathrm{O}_{2}{ }^{-}\right)^{1}$. ROS can directly damage cells via a number of mechanisms e.g. by influencing the opening probability of the mitochondrial permeability transition pore ${ }^{11}$, by causing lipid peroxidation ${ }^{1}$, by activating matrix metalloproteinases (MMP's) ${ }^{12}$, or by oxidising DNA ${ }^{12}$. However, ROS may also damage cells in a more indirect manner: They can e.g. interact with nitric oxide (NO), fatty acids or free iron (Fenton reaction). This often results in the formation of even more cytotoxic substances, such as peroxynitrite, peroxyl radicals and hydroxyl radicals ${ }^{11}$. In addition, ROS can enhance the inflammatory response by upregulation of chemokines and adhesion molecules ${ }^{1,10}$. Thus, the reperfusion phase can be seen as a 'double-edged sword' ${ }^{13}$ : On the one hand there is a cellular demand for the replenishment of oxygen, while on the other hand this oxygen results in ROS formation. The paradoxical role of oxygen in the first two phases is called the 'oxygen paradox' 9 . The second, ROS paradox shows up in a later and more chronic phase of IRI, the post-reperfusion phase. In contrast to their detrimental role in the acute phases, here ROS affect several tightly regulated processes that lead to an optimal environment for survival: Angiogenesis is induced by ROS through upregulation or activation of vascular endothelial growth factor (VEGF) and hypoxia inducible factor (HIF) ${ }^{14,15}$. The proliferation and differentiation of vascular smooth muscle cells, needed for vascular remodelling, are also affected by ROS ${ }^{14,15}$. These beneficial roles of ROS are also seen during permanent ischemia.

\section{NADPH oxidase as a source of ROS in IRI}

Most sources of ROS (see 'other sources of ROS in IRI') only generate ROS 'by accident', i.e. as a by-product of the metabolism they are involved in or during nonphysiologic conditions such as ischemia ${ }^{16-21}$. NADPH oxidases stand out from these sources of ROS, as they constitute the only enzyme family with the sole function to produce ROS, not only in disease, but also in physiology ${ }^{22}$. NADPH oxidases are abundantly expressed in the vasculature, where they are emerging as the key producers of ROS ${ }^{7,23-26}$. 
Depending on the phase of IRI, NADPH oxidases can be either detrimental or protective. Thus, NADPH oxidases also have a double-edged role, similar to their substrate, oxygen.

Interestingly, several drugs used to treat cardiovascular diseases regulate the expression and activity of NADPH oxidases. For example, statins prevent the translocation of the NADPH oxidase subunit Rac1 to the cell membrane ${ }^{27}$. This translocation is one of the essential steps in activation of some NOX isoforms ${ }^{28}$. Thus, some of the pleiotropic effects of statins can be attributed to their ability to decrease NADPH oxidase mediated ROS production ${ }^{27,29,30}$. Angiotensin-convertingenzyme (ACE) inhibitors and angiotensin receptor blockers (ARB) reduce angiotensin II formation and angiotensin II mediated AT1-receptor activation, respectively. Since angiotensin II signalling is a potent stimulus for NADPH oxidase expression and activity ${ }^{29,30}$, ACE inhibitors and ARB decrease NADPH oxidase expression and activation.

\section{Biochemistry of NADPH oxidases}

NADPH oxidases are multi-protein complexes. Seven isoforms of their catalytic subunits exist, annotated as NOX1-5 and DUOX1-2 (recently also termed NOX6-7). They contain six or seven, respectively, trans-membrane spanning domains. They catalyse the electron transport from NADPH to molecular oxygen, thereby producing ROS $7,22,31,32$. All isoforms have two heme groups, an FAD and a NADPH binding site $^{26}$. NOX2 was the first described isoform. It was discovered in neutrophils, where it mediates oxidative burst and is thus important for innate host defence ${ }^{22-24,28}$. In the vasculature, NOX2 is active in almost all vascular wall cells ${ }^{33,34}$. In the cardiovascular system, next to NOX2, also NOX1, NOX4, and NOX5 are expressed. The latter, however, is not present in rats and mice ${ }^{24,28,32}$. NOX1 is expressed in endothelial cells, VSMC and adventitial fibroblasts ${ }^{35}$. NOX4, the most abundantly expressed isoform, is found in endothelial cells and VSMC ${ }^{24,35}$. Its expression and activity seem to be especially prominent in the cerebral vasculature ${ }^{36}$, with genderspecific differences ${ }^{37}$. NOX5 is located in vascular endothelial cells ${ }^{38}$ and VSMC ${ }^{39}$. The NOX isoforms not only vary in their expression pattern but also in their subcellular localisations. 
For example, NOX4, is found in the nucleus, and might be present in mitochondria ${ }^{40}$, making NOX4 a potential source of mitochondrial ROS. NOX1 co-localises with caveolin-1 ${ }^{41}$, associating it with caveolae and eNOS uncoupling (see below) ${ }^{42}$. Furthermore, the various NOX isoforms require different cytosolic subunits for activity, such as activator and organiser proteins or Rac1 ${ }^{43,44}$. NOX4 stands out from the other NOX isoforms as it is constitutively active and produces $\mathrm{H}_{2} \mathrm{O}_{2}$ as its main product ${ }^{28}$. An important feature of NOX5 is that it does not need any of the cytosolic subunits. Importantly, it is directly activated by calcium through calcium binding to its EF hand motifs ${ }^{24}$. NOX6-7 (formerly called DUOX1-2) probably play no (major) role in the vasculature. Fig. 1 and Table 1 give an overview of the characteristics of the isoforms expressed in the cardiovascular system.

\section{Validation of NADPH oxidases as therapeutic targets for IRI}

\section{NADPH oxidases during acute ischemia}

NADPH oxidases have been proposed to be oxygen sensors and thus to be involved in the ischemic phase of IRI ${ }^{46-49}$. They are also likely to interact with the HIF-pathway (see below) ${ }^{50-56}$.

Oxygen sensing is the endogenous ability of tissues to respond to changes in oxygen tension. It occurs in specialized cells such as the glomus cells of the carotid body and the neuroepithelial bodies (NEB) in the lung. While NADPH oxidases are expressed in these tissues ${ }^{48,49}$, a true functional role in oxygen sensing is disputed ${ }^{48,49,57-59}$. In this review, we focus on true IRI; discussing the mechanisms of oxygen sensing in more detail is beyond the scope of this review.

First evidence for a role of NADPH oxidases in ischemia comes from studies showing an altered expression on mRNA or protein levels of NADPH oxidases caused by a change in oxygen tension. In pulmonary artery smooth muscle cells (PASMC), endothelial cells, pulmonary epithelial cells and PC12 cells, a cell line from pheochromocytome of rat adrenal medulla $52,54,55$, either NOX1, NOX2 or NOX4 mRNA and protein levels are upregulated during hypoxia. 

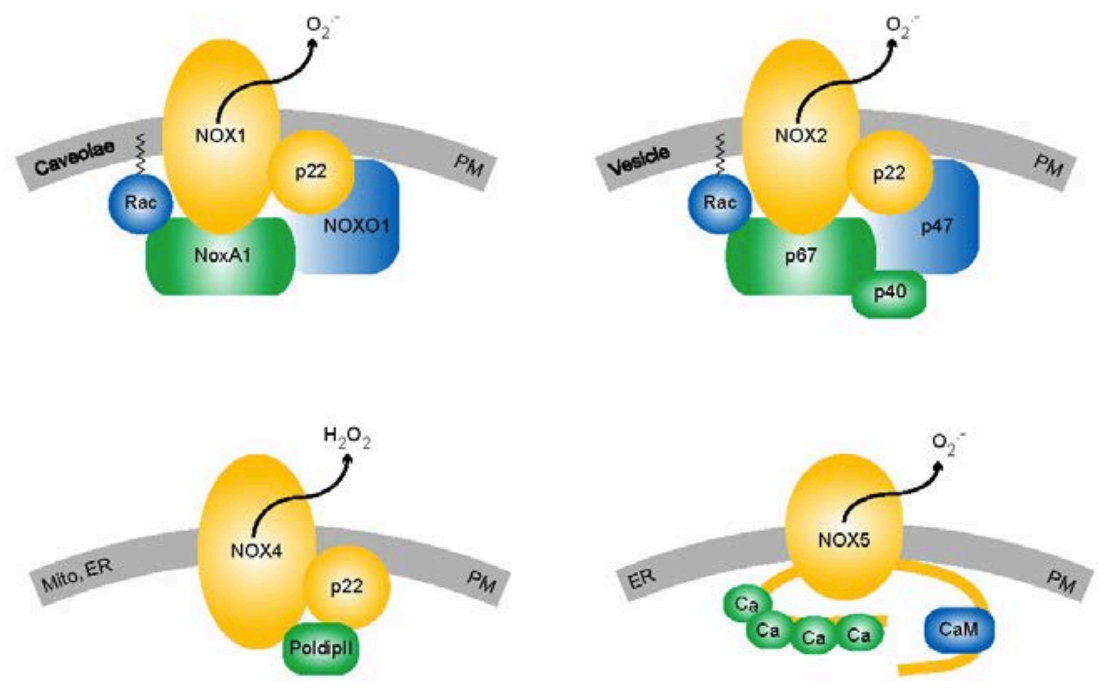

Modified from [44]

Figure 1. The four vascular NOX isoforms need different subunits for their activation, have different subcellular localisations and produce different types of ROS. NOX2 is the first described isoform of the NADPH oxidase family. It consists of a six-trans-membrane spanning catalytic domain. The membrane-bound subunit $p 22^{\text {phox }}$ and cytosolic subunits $p 47^{\text {phox }}, p 67^{\text {phox }}$, and $p 40^{\text {phox }}$ need to assemble for NOX2 activation. In addition, the small Gprotein Rac binds to this complex. NOX2 is mainly localised in intracellular vesicles, but also in plasma membranes (PM). The basic structure of NOX1 is similar to NOX2. However, NOX1 interacts with the $p 47^{\text {phox }}$ and $p 67^{\text {phox }}$ analogues, NOXO1 and NOXA1, although at least in recombinant systems, it is also active upon interaction with $p 47^{\text {phox }}$ and $p 67^{\text {phox }}$ (not shown). NOX1 activity also depends on $p 22^{\text {phox }}$ and Rac. Similar to NOX2, NOX1 is located in the PM. In addition, it was suggested to be located in caveolae. NOX3 appears not to be expressed in the vasculature but the inner ear ${ }^{28,31}$. NOX4 is different from the other vascular isoforms in that it only interacts with $p 22^{\text {phox }}$ but does not rely on other cytosolic subunits for its activation. It was suggested that NOX4 is not only located in the plasma membrane, but also in membranes of the endoplasmic reticulum (ER) and in mitochondria. NOX4 is constitutively active, but can be further activated by several factors such as angiotensin or growth factors. Poldip2 has been described as a binding protein that can activate NOX4 ${ }^{45}$. NOX4 has been suggested to also or even predominantly generate hydrogen peroxide $\left(\mathrm{H}_{2} \mathrm{O}_{2}\right)$. NOX5 has mainly been found in the ER in addition to its localisation in the PM. It produces superoxide and is active without any subunits. An increase in calcium activates NOX5 mediated by its calcium binding domains, i.e. the EF hands. In addition, the Cterminus of NOX5 has a calmodulin binding-domain. 
Table 1. Specifications of the different NADPH oxidase isoforms (adapted from Radermacher et al).

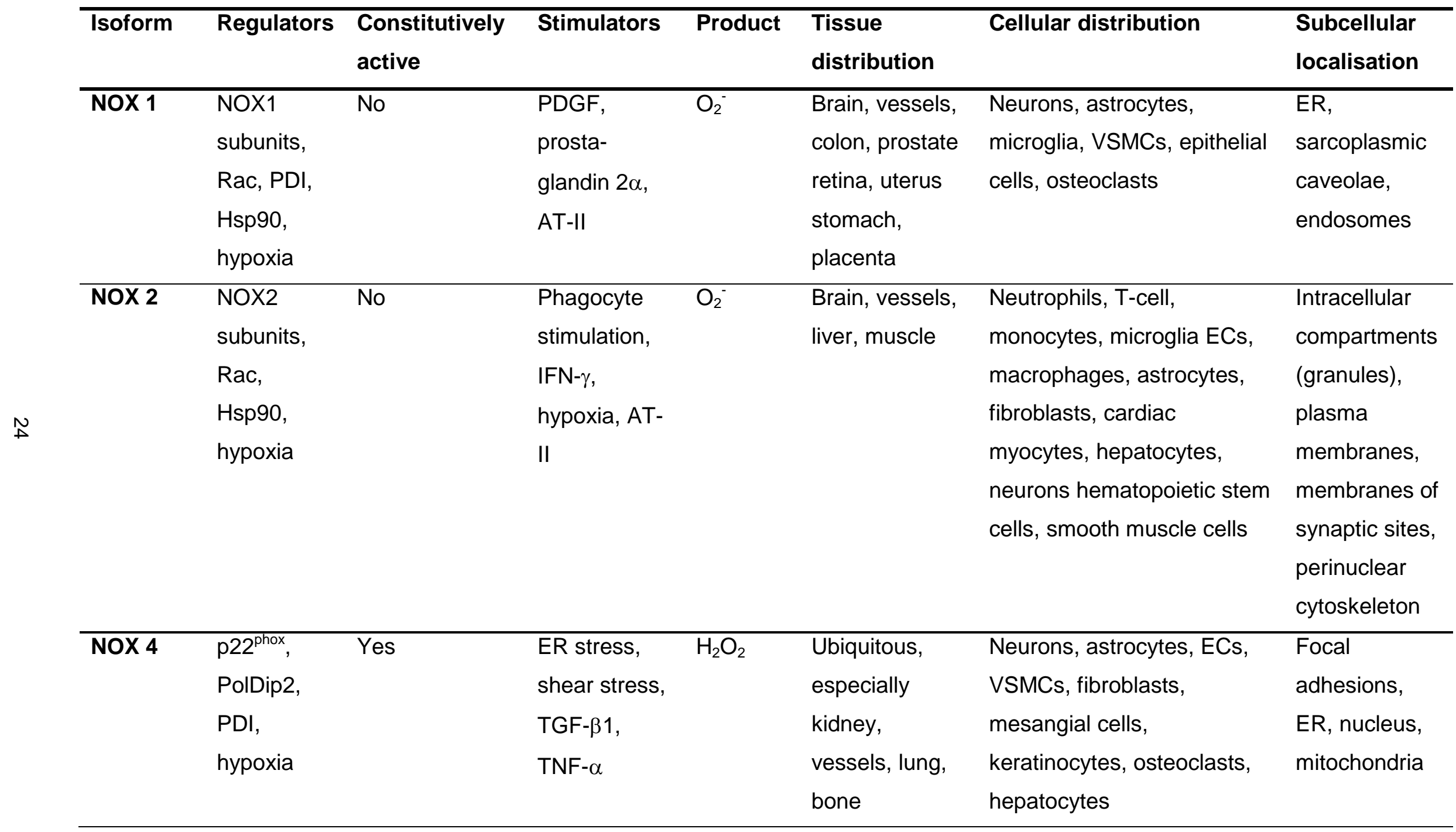


Table 1, continued

\begin{tabular}{|c|c|c|c|c|c|}
\hline NOX 5 & $\begin{array}{l}\text { no } \\
\text { subunits, } \\
\text { but calcium } \\
\text { sensitive, } \\
\text { Hsp90 }\end{array}$ & $\uparrow$ calcium & $\mathrm{O}_{2}^{-}$ & $\begin{array}{l}\text { Testis, spleen, } \\
\text { kidney, lymphatic } \\
\text { tissue, uterus }\end{array}$ & $\begin{array}{l}\text { ECs, VSMCs, lymphocytes, ? } \\
\text { and several cancer cell lines }\end{array}$ \\
\hline
\end{tabular}

AT-II, angiotensin-II; ECs, endothelial cells; ER, endoplasmic reticulum; $\mathrm{H}_{2} \mathrm{O}_{2}$, hydrogen peroxide; Hsp90: heat shock protein 90; $\mathrm{O}_{2}^{-}$, superoxide, IFN- $\gamma$, interferon- $\gamma$, PDI: protein disulphide isomerase; PolDip2: polymerase (DNA-directed) delta interacting protein; TGF- $\beta 1$, transforming growth factor- $\beta 1$; TNF- $\alpha$, tumor necrosis factor $\alpha ;$ VSMCs, vascular smooth muscle cells. 
In vivo, an upregulation of NOX4 was also shown in mouse lungs after hypoxia ${ }^{52,60}$. Similarly, mRNA levels of the NADPH oxidase subunits $\mathrm{p} 22^{\text {phox }}, \mathrm{p} 47^{\text {phox }}$ and $\mathrm{p} 67^{\text {phox }}$ increased in response to hypoxia in human umbilical vein endothelial cells (HUVEC) ${ }^{61}$. In the brain of rats and mice, upregulation of NOX2, NOX4, and p4 $7^{\text {phox }}$ mRNA levels were reported after stroke induced by $1 / R^{62-64}$. An increase in NOX2, NOX4, p22 ${ }^{\text {phox }}$, and p $67^{\text {phox }}$ mRNA was also found after myocardial infarction in mice 65-67. Furthermore, NOX4 mRNA and protein levels were upregulated in peripheral muscles after hindlimb ischemia in mice ${ }^{68}$. Taken together, the aforementioned studies indicate that NOX isoforms are upregulated at the mRNA level. However, a concomitant increase at the protein or functional level has not always been reported. Therefore, further studies are necessary.

The effects of hypoxia on NADPH oxidases and reciprocal effects of NADPH oxidases on hypoxic signalling pathways seem to be mediated by different regulatory pathways, some of them involving hypoxia-inducible transcription factors of the HIF family ${ }^{69}$. HIFs are composed of an oxygen-sensitive $\alpha$-subunit and a constitutively expressed $\beta$-subunit also termed aryl hydrocarbon receptor nuclear translocator $(\text { ARNT })^{70}$.

Interactions between NADPH oxidases and HIF proteins have been shown for different NOX isoforms and NADPH oxidase subunits ${ }^{69,71}$. ROS derived from NADPH oxidases can upregulate HIF-1 $\alpha$ mRNA and protein levels ${ }^{72}$. In VSMC, HIF$1 \alpha$ is induced by thrombotic factors. This induction and its further activation depend on Rac1 and $\mathrm{p} 22^{\text {phox } 53,73}$. Further data indicate that NOX2 and NOX4 play a role in regulating HIF-1 $\alpha$ levels in endothelial cells, thereby modulating angiogenic activity ${ }^{74}$. In PASMC, NOX4 induced HIF-1 $\alpha$ and HIF-2 $\alpha$ levels by interfering with the activity of the HIF- $\alpha$ modifying prolyl hydroxylases ${ }^{51}$. These data indicate that NADPH oxidases can modulate HIF activity by several mechanisms including transcriptional upregulation of $\mathrm{HIF}-1 \alpha$ through the activation of $\mathrm{NFKB}^{72}$. In addition, NADPH oxidases have been reported to mediate post-translational stabilization of HIF-1 $\alpha$ proteins by interfering with the HIF degrading machinery ${ }^{51}$.

Both transient and stable overexpression of NOX1 in A549 cells resulted in increased HIF-1-dependent target gene expression ${ }^{54}$. Also, increased HIF-1 $\alpha$ protein was found both during hypoxia and normoxia in these NOX1-transfected cells ${ }^{54}$. 
On the other hand, expression of active Rac1 decreased nuclear HIF-1 $\alpha$ levels in HepG2 cells and primary rat hepatocytes under hypoxia ${ }^{75}$ while opposite effects were reported in Hep3B cells ${ }^{76}$. The different NOX isoforms/subunits might thus have specific roles, and/or the redox sensitivity of different cells and tissues might be specific.

NADPH oxidases not only regulate HIF-1 $\alpha$ and HIF-2 $\alpha$, but HIF can vice versa also affect NADPH oxidases. In mice with a heterozygous defect in HIF-1 $\alpha$ and thus reduced HIF-1 $\alpha$ levels, NOX2 mRNA and protein did not increase in cortex and brainstem after exposure to intermittent hypoxia, while in WT mice NOX2 was upregulated ${ }^{55}$. Fibroblasts from HIF-1 $\alpha$ heterozygous mice had a lower basal HIFdependent reporter gene activity, and NOX2 mRNA levels did not increase after hypoxia, but they did increase in fibroblasts from WT mice ${ }^{55}$. Similarly, in endothelialspecific HIF-1 $\alpha$ knockout mice, NOX2 mRNA expression and protein levels were reduced even when adding the NOX2-stimulator urotensin $\mathrm{II}^{74}$.

Overexpression of HIF-1 $\alpha$ in PC12 cells also increased NOX2 mRNA and protein levels as well as NADPH oxidase activity after intermittent hypoxia ${ }^{56}$. Importantly, recently a functional HIF binding site in the proximal promoter of the NOX2 gene was identified ${ }^{74}$, and thus, NOX2 was added to the list of genuine HIF-1 target genes. As such, HIF-1 can regulate the expression of NOX2 and thereby ROS production by NOX2. For NOX4, a decrease of its mRNA levels in PASMC during hypoxia after treatment of the cells with shRNA against HIF-1 $\alpha$ was reported ${ }^{52}$. Vice versa, overexpression of HIF-1 $\alpha$ resulted in elevated NOX4 mRNA and protein levels. ROS production also increased after overexpression of HIF-1 $\alpha$, but only when NOX4 was present ${ }^{52}$. Similarly to NOX2, a functional HIF binding site was identified in the NOX4 promoter thus adding also NOX4 to the list of direct HIF target genes ${ }^{52}$.

These findings suggest that ROS-dependent activation of HIF can induce expression of several NADPH oxidase subunits under normoxic conditions, thus promoting ROSmediated signalling pathways. Under low oxygen conditions, such as ischemia, the rapid stabilisation and activation of HIF may be the primary event responsible for upregulation of NADPH oxidases. This process may help cells and tissues to adapt at least partially to the decreased substrate availability under these conditions, thereby allowing a certain restoration of ROS levels also under hypoxia. 
However, whether NADPH oxidases can also be activated under hypoxia without concomitant increase in subunit availability is not clear, and precise mechanisms and interactions of NADPH oxidase derived ROS under these conditions still need to be elucidated.

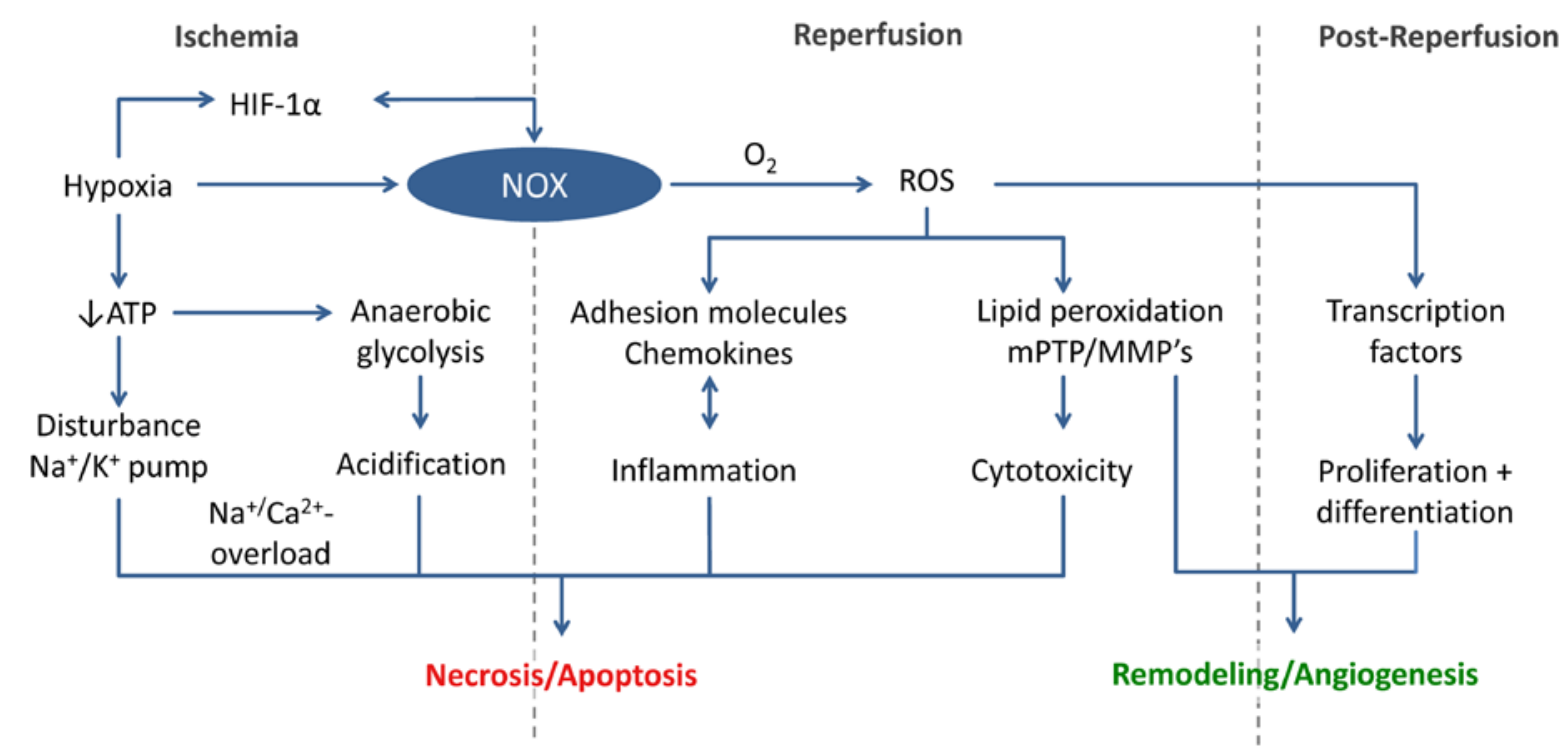

Figure 2. The role of NADPH oxidases in the three phases of IRI. During the ischemic phase, the lack of oxygen results in decreased ATP production, increasing the calcium concentration, which ultimately results in necrosis. In addition, the drop in oxygen upregulates NADPH oxidases via stimulation of HIF-1 $\alpha$. NADPH oxidases can also activate HIF-1 $\alpha$, initiating a positive feedback loop. When a re-introduction of oxygen occurs during the reperfusion phase, NADPH oxidases produce large amounts of reactive oxygen species (ROS). These ROS then enhance the inflammatory response by upregulation of adhesion molecules and chemokines, which activate and attract leucocytes. Inflammation and edema ensue. ROS can also have direct cytotoxic effects. Together with the inflammation, this ultimately results in necrosis and apoptosis of cells. ROS production also occurs during the post-reperfusion phase. In this phase, a certain level of ROS is needed to ensure a proper stimulation of angiogenesis via VEGF activating endothelial cells (EC) and remodelling via the NFKB pathway activating vascular smooth muscle cells (VSMC). This leads to a better environment for tissue survival. 


\section{NADPH oxidases during reperfusion}

Upon re-introduction of oxygen, NADPH oxidases are believed to generate large amounts of ROS, which have direct cytotoxic effects ${ }^{11,12}$.

For example, after I/R of the lung or the brain, lipid peroxidation, protein nitration and oxidative DNA damage was smaller in NOX2 $\mathrm{KO}^{77}$ or $\mathrm{p} 47^{\mathrm{phox}} \mathrm{KO}$ mice compared to WT mice ${ }^{78,79}$. Similar results were found when apocynin, an unspecific NADPH oxidase inhibitor ${ }^{30,80}$, was applied to WT mice ${ }^{77,79}$. Apocynin and DPI, another unspecific NADPH oxidase inhibitor, also reduced the increases in lipid peroxidation, cell death and apoptosis after I/R in H9c2 cells ${ }^{81,82}$. However, these results should be interpreted with caution, because of the lack of specificity of these inhibitors, such as antioxidant properties of apocynin ${ }^{80}$, and DPI is a general flavoprotein inhibitor ${ }^{83}$. The more specific NADPH oxidase inhibitor VAS2870 given to WT mice two and 12 hours after cerebral I/R, which was induced by IMCAO, decreased infarct size, ROS levels, tissue nitration, apoptosis and brain oedema. VAS2870 also improved functional outcome in WT mice after induction of ischemic strokes ${ }^{63}$. NOX4 KO mice showed the same decreases in damage as VAS2870 treated WT mice, while treatment of NOX4 KO mice with VAS2870 did not have an additional protective effect ${ }^{63}$. This strongly suggests that NOX4 contributes to cerebral IRI. Together, these studies show that NADPH oxidases-derived ROS, particularly NOX4, are likely to be involved in the direct cytotoxic effects of IRI in the brain.

Evidence for the role of other NOX isoforms in cerebral IRI is contradictory. No effect of NOX1 or NOX2 in brain IRI was found in the study mentioned above ${ }^{63}$. Two other studies showed no role ${ }^{84}$ or a detrimental role ${ }^{85}$, respectively, of NOX1 in stroke. Data concerning the role of NOX2 are also conflicting ${ }^{63,77,78,85-88}$. For a more detailed discussion of the role of NADPH oxidases in brain I/R, we refer to ${ }^{89}$.

NADPH oxidases can also indirectly cause damage by enhancing the inflammatory response ${ }^{22}$. Neutrophils, which express NOX2, are the primary source of ROS after heart $\mathrm{I} / \mathrm{R}$ in dogs ${ }^{90}$. Further, neutrophil infiltration was reduced after brain $\mathrm{I} / \mathrm{R}$ in NOX2 KO mice compared to WT mice. Similarly, neutrophil infiltration was attenuated in lungs of $\mathrm{p} 47^{\text {phox }} \mathrm{KO}$ mice or of mice treated with apocynin ${ }^{77,79}$. 
However, again, apocynin is not specific for NADPH oxidases. Furthermore, after lung I/R, pro-inflammatory cytokine levels including TNF- $\alpha$, IL-6, IL-1 $\beta$ and MCP-1 in lungs were lower in $\mathrm{p} 47^{\text {phox }} \mathrm{KO}$ mice compared to WT mice ${ }^{79}$. This was also observed in the brain after $\mathrm{I} / \mathrm{R}{ }^{77}$. Here, the ischemic stroke induced disruption of the blood-brain-barrier, which contributes to inflammation via increased permeability and subsequent tissue swelling after brain I/R. This blood-brain barrier disruption was reduced in NOX2 KO mice compared with WT mice ${ }^{87}$.

In an isolated perfused lung I/R model, edema and vascular permeability were reduced in lungs from NOX2 KO mice, but neither in lungs from NOX1 KO mice, nor in NOX4 KO mice lungs ${ }^{91}$.

Together these data suggest a role of NOX2 or p47 phox in the inflammatory reaction and chemotaxis during IRI. The regulation of different cytokines in different organs suggests a cell- or organ-specific effect of NOX2.

With respect to the other NOX isoforms, no solid data on their involvement in inflammation and chemotaxis after IRI are available.

\section{NADPH oxidases during post-reperfusion and chronic ischemia}

ROS are not only toxic during acute IRI, they are also key signalling molecules in pathways mediating cell proliferation and differentiation ${ }^{7,22,92-94}$, which are needed for both angiogenesis and remodelling. During the post-reperfusion period or during chronic ischemia, NADPH oxidases might thus have a protective role, showing the second edge of the NADPH oxidase sword ${ }^{95-98}$. On the one hand, ROS can mediate auto-phosphorylation of vascular endothelial growth factor receptor 2 (VEGFR2), which leads to activation of downstream signalling pathways ${ }^{97}$. In addition, by interacting with HIF-1 $\alpha$, ROS can activate VEGF and other targets, which protect and activate vascular proliferative responses such as plasminogen activator inhibitor 1 (PAl-1) 69,71,72.

On the other hand, VEGF can activate NADPH oxidases via Rac1 leading to increased ROS production ${ }^{99}$. This feedback loop ultimately results in increased VEGF-induced angiogenesis. In non-vascular cells, there is also a negative feedback loop involved, in which Rac1 inhibits HIF-1 and PAI-1 and thus decreases angiogenesis ${ }^{75}$. 
The non-specific NADPH oxidase inhibitor, DPI, and the antioxidant, $\mathrm{N}$-acteyl cysteine (NAC), decreased VEGF-induced ROS levels in HUVEC, as did NOX2 antisense constructs ${ }^{99} \cdot \mathrm{p} 47^{\text {phox }}$ downregulation in HUVEC also led to decreased VEGF mediated phosphorylation of downstream mediators ${ }^{100}$. VEGF-induced neovascularisation in a sponge implant model was also reduced in NOX2 KO mice compared to WT mice ${ }^{99}$. Together, these data clearly point to a role of NADPH oxidases in the VEGF signalling pathway in vascular cells at several levels. However, the importance of NOX2 for angiogenic responses is not limited to VEGF signalling. Recently, it was reported that the vasoactive peptide urotensin-II can also induce angiogenesis, and this response was inhibited in vitro by downregulating NOX2 in endothelial cells using shRNA. Similarly, vascular sprouting into a matrigel plug was disrupted in NOX2 KO, but not in WT mice ${ }^{74}$.

After hindlimb ischemia, flow recovery and capillary density were reduced in NOX2 KO mice ${ }^{101,102}$. However, there is also evidence for a normal collateral growth in NOX2 KO mice, with only a dysfunction of the newly formed vessels, resulting in compromised perfusion ${ }^{103}$. In addition, when adding oxidative stress triggers, such as smoking or diabetes, the impairment of new vascularisation was less pronounced in NOX2 KO compared to WT mice ${ }^{104,105}$. Thus, while NOX2 clearly seems to be involved in promoting angiogenesis in vivo, it is the specific environment (i.e. oxidative stress or not) that finally determines the overall impact of NOX2 on these responses.

During remodelling in the chronic phase of IRI, NOX2 seems to play a detrimental role. NOX2 or $\mathrm{p} 47^{\text {phox }} \mathrm{KO}$ mice subjected to myocardial infarction displayed less dilatation of the left ventricle, less hypertrophy and less interstitial fibrosis along with an increased survival after 4 weeks ${ }^{65,66}$. Interestingly, infarct sizes did not differ between KO and WT mice both after 24 hours and 4 weeks ${ }^{65,66}$. Others did not find any difference between NOX2 KO and WT mice in infarct size, apoptosis, collagen content or inflammation after myocardial infarction ${ }^{67,106}$. However, some neither used littermates as control mice nor sham-operated animals. This is relevant when interpreting these results. Furthermore, differences in mouse strains can influence heart remodelling ${ }^{107}$. Also, $\mathrm{p} 47^{\text {phox }} \mathrm{KO}$ and $\mathrm{p} 47^{\text {phox }}$ heterozygous mice had similar infarct sizes and left ventricular functions $24 \mathrm{~h}$ after I/R of the heart ${ }^{108}$. 
However, 24h of reperfusion in the heart can be rather regarded as an acute reperfusion phase; thus, these results suggest that there is no role of NOX2 and $\mathrm{p} 47^{\text {phox }}$ in acute heart IRI. Rather, there may be an effect in the post-reperfusion phase.

Although the data are contradictory, most studies suggest a rather detrimental role of NOX2 in long-term remodelling after myocardial infarction.

NOX1 has been shown to be involved in angiogenesis in tumours ${ }^{109}$, but data in I/R models are missing. NOX1 overexpressing transgenic mice showed an increased hypertrophy of VSMC in response to angiotensin-II ${ }^{110}$. Moreover, NOX1 may be involved in cell growth and transformation, not only in the vasculature but also in tumor forming cells ${ }^{111,112}$.

For NOX4, data suggest a protective role in the chronic phase of IRI. siRNA against NOX4 reduced tube formation and wound healing responses in HMEC and HUVEC, and also inhibited both basal and VEGF-induced cell migration or proliferation ${ }^{68,113}$. NOX4 overexpression increased these responses ${ }^{68,113}$. In line, similar responses of cell migration and proliferation were found after overexpressing or silencing NOX4 in human and bovine endothelial cells grown on matrigel ${ }^{68}$. However, studies using siRNA against NOX4 should be interpreted with caution, since not all siRNAs specifically down-regulate NOX4, and some may also affect other NOX isoforms ${ }^{114}$. In mice injected with adenoviral NOX4 into the hindlimb, blood flow was increased after ligation of the femoral artery compared to non-treated animals ${ }^{68}$. Accordingly, blood flow restoration in NOX4 KO mice was reduced after hindlimb ischemia ${ }^{115}$. Furthermore, aortas of endothelial-specific NOX4 transgenic mice displayed increased sprouting ex vivo ${ }^{68}$. Thus, a protective role for NOX4 in angiogenesis during chronic ischemia is likely.

In addition, an involvement of NOX4 in the regulation of VSMC growth and differentiation was suggested ${ }^{92,94}$. In wound healing and embryogenesis, maintenance of VSMC differentiation might be a beneficial effect of NOX4 ${ }^{92}$. However, in IRI and cardiac pressure overload, NOX4 in cardiomyocytes and cardiac fibroblasts may have a more ambivalent role: They seem to stimulate proliferation and differentiation of cardiac fibroblasts into myofibroblasts, which may lead to fibrosis, cardiac remodelling and heart failure ${ }^{93,116}$. 
In human PASMC, NOX4 also mediated proliferation in response to hypoxia, but also to factors such as transforming growth factor $\beta 1$ or urotensin-II, which can contribute to vascular remodelling in different disease states ${ }^{52,117,118}$. Moreover, hypertrophic stimuli such as angiotensin II and pressure overload increased NOX4 expression in cardiac myocytes ${ }^{119}$. Aged mice overexpressing NOX4 in the heart showed increased fibrosis and apoptosis of cardiomyocytes ${ }^{119}$. Interestingly, overexpression of HIF-1 $\alpha$ increased proliferation in PASMC, but not when NOX4 was depleted by ShRNA ${ }^{52}$. Thus an additional role of HIF-1 $\alpha$ in the VSMC proliferative response seems likely. Altogether, NOX4 seems to be involved in regulating growth and differentiation of VSMC and cardiac cells, which in the post-reperfusion phase might lead to remodelling and formation of fibrosis. Unfortunately, there are no data on the effect of NOX4 on the long-term remodelling after IRI.

The in vivo and in vitro studies discussed are summarised in Table 2 and 3 , respectively. 
Table 2. Overview of the in vivo studies showing a role of NADPH oxidases in different forms of ischemia-reperfusion injury.

\begin{tabular}{|c|c|c|c|c|c|c|c|c|}
\hline \multicolumn{9}{|l|}{ Heart } \\
\hline \multirow[t]{2}{*}{ Animal model } & \multirow{2}{*}{$\begin{array}{l}\text { Ischemic } \\
\text { model }\end{array}$} & \multirow{2}{*}{$\begin{array}{l}\text { Timepoint } \\
\text { outcome }\end{array}$} & \multicolumn{5}{|c|}{ Outcomes } & \multirow[t]{2}{*}{ Ref } \\
\hline & & & $\begin{array}{l}\text { Cardiac } \\
\text { dilatation }\end{array}$ & $\begin{array}{l}\text { Cardiac } \\
\text { dysfunction }\end{array}$ & Infarct size & Survival & Inflammation & \\
\hline $\begin{array}{l}\mathrm{p} 47^{\text {phox }} \mathrm{KO} \text { vs WT } \\
\text { mice }\end{array}$ & LAD ligation & 4 weeks & $\downarrow$ & $\downarrow$ & $\varnothing$ & $\uparrow$ & n.a & 64 \\
\hline $\begin{array}{l}\mathrm{p} 47^{\text {phox }} \mathrm{KO} \text { vs } \mathrm{p} 47^{\text {phox }} \\
\text { Het mice }\end{array}$ & $\begin{array}{l}\text { Transient LAD } \\
\text { ligation ( } 30 \\
\text { min) }\end{array}$ & $24 \mathrm{~h}$ & $\varnothing$ & $\varnothing$ & $\varnothing$ & n.a & $\uparrow$ & 107 \\
\hline NOX2 KO vs WT mice & LAD ligation & 8 weeks & $\varnothing$ & $\varnothing$ & $\varnothing$ & $\downarrow$ & n.a & 105 \\
\hline NOX2 KO vs WT mice & LAD ligation & 4 weeks & $\downarrow$ & $\downarrow$ & $\varnothing$ & $\varnothing$ & n.a & 65 \\
\hline NOX2 KO vs WT mice & LAD ligation & 4 weeks & n.a & n.a & $\varnothing$ & n.a & $\varnothing$ & 66 \\
\hline \multicolumn{9}{|l|}{ Brain } \\
\hline \multirow[t]{2}{*}{ Animal model } & Ischemic & Time & \multicolumn{5}{|c|}{ Outcomes } & $\overline{R e f}$ \\
\hline & model & endpoint & Infarct size & $\begin{array}{l}\text { Neurological } \\
\text { outcome }\end{array}$ & Inflar & mation & $\begin{array}{l}\text { BBB- } \\
\text { dysfunction }\end{array}$ & \\
\hline NOX1 KO vs WT mice & $\begin{array}{l}\text { tMCAO } \\
\text { (30min) }\end{array}$ & $24 \mathrm{~h}$ & $\varnothing$ & $\varnothing$ & n.a & & n.a & 83 \\
\hline NOX1 KO vs WT mice & $\begin{array}{l}\text { tMCAO } \\
\text { (60min) }\end{array}$ & $24 \mathrm{~h}$ & $\varnothing$ & $\varnothing$ & n.a & & n.a & 62 \\
\hline
\end{tabular}


Table 2, continued

\begin{tabular}{|c|c|c|c|c|c|c|c|}
\hline $\begin{array}{l}\text { NOX1 KO vs WT } \\
\text { mice }\end{array}$ & tMCAO (60min) & & $\downarrow$ & $\uparrow$ & n.a & $\downarrow$ & 84 \\
\hline $\begin{array}{l}\text { NOX2 KO vs WT } \\
\text { mice }\end{array}$ & tMCAO (60min) & 24 and $72 \mathrm{~h}$ & $\downarrow$ & n.a & $\downarrow$ & n.a & 77 \\
\hline NOX2 KO mice & tMCAO (75min) & 24 and $72 \mathrm{~h}$ & $\downarrow$ & $\uparrow$ & $\downarrow$ & n.a & 76 \\
\hline $\begin{array}{l}\text { NOX2 KO vs WT } \\
\text { mice }\end{array}$ & tMCAO (25min) & $72 \mathrm{~h}$ & $\downarrow$ & n.a & n.a & n.a & 151 \\
\hline $\begin{array}{l}\text { NOX2 KO vs WT } \\
\text { mice }\end{array}$ & tMCAO (120min) & $1 / 2 / 24 h$ & $\begin{array}{l}24 \mathrm{~h}: \downarrow \quad 2 \mathrm{~h}: \\
\varnothing\end{array}$ & n.a & n.a & 1h: $\downarrow$ & 86 \\
\hline $\begin{array}{l}\text { NOX2 KO vs WT } \\
\text { mice }\end{array}$ & $\begin{array}{l}\text { tMCAO } \\
(60 \mathrm{~min})\end{array}$ & $24 \mathrm{~h}$ & $\varnothing$ & $\varnothing$ & n.a & n.a & 62 \\
\hline $\begin{array}{l}\text { NOX2 KO vs WT } \\
\text { mice }\end{array}$ & tMCAO (120min) & $24 \mathrm{~h}$ & $\downarrow$ & n.a & $\varnothing$ & n.a & 87 \\
\hline NOX4 KO vs WT & tMCAO (60min) & $24 \mathrm{~h}$ & $\downarrow$ & $\uparrow$ & n.a & $\downarrow$ & 62 \\
\hline \multirow[t]{2}{*}{ mice } & pMCAO & $24 h$ & $\downarrow$ & $\uparrow$ & n.a & n.a & \\
\hline & PT & $24 \mathrm{~h}$ & $\downarrow$ & n.a & n.a & n.a & \\
\hline $\begin{array}{l}\text { WT mice treated } \\
\text { with VAS } 2870\end{array}$ & tMCAO & $24 h$ & $\downarrow$ & $\uparrow$ & n.a & n.a & \\
\hline
\end{tabular}


Table 2, continued

\begin{tabular}{|c|c|c|c|c|c|c|}
\hline \multicolumn{7}{|l|}{ Hindlimb } \\
\hline \multirow[t]{3}{*}{ Animal model } & \multirow[t]{3}{*}{ Ischemic model } & \multirow[t]{3}{*}{ Time endpoint } & \multicolumn{3}{|c|}{ Outcomes } & \multirow[t]{3}{*}{ Ref } \\
\hline & & & Blood flow & Capillary density & Ischemic & \\
\hline & & & recovery & & muscle injury & \\
\hline NOX1 KO vs WT mice & FA ligation & 2 weeks & $\uparrow$ & n.a & n.a & 114 \\
\hline NOX2 KO vs WT mice & FA excision & 2 weeks & $\downarrow$ & n.a & $\uparrow$ & 102 \\
\hline NOX2 KO vs WT mice & FA ligation & 3 weeks & $\downarrow$ & $\downarrow$ & n.a & 104 \\
\hline Diabetic NOX2 KO vs diabetic WT mice & & & $\uparrow$ & $\uparrow$ & n.a & \\
\hline NOX2 KO vs WT mice & FA ligation & 2 weeks & $\downarrow$ & n.a & n.a & 114 \\
\hline NOX2 KO vs WT mice & FA excision & 2 weeks & $\uparrow$ & $\uparrow$ & n.a & 103 \\
\hline $\begin{array}{l}\text { NOX2 KO vs WT mice after chronic } \\
\text { smoke exposure }\end{array}$ & & & $\uparrow$ & $\uparrow$ & n.a & \\
\hline NOX2 KO vs WT mice & FA ligation & 1 week & $\downarrow$ & $\downarrow$ & n.a & 101 \\
\hline NOX2 KO vs WT mice & FAV excision & 2 weeks & $\downarrow$ & n.a & n.a & 100 \\
\hline $\begin{array}{l}\text { WT mice locally injected with adenoviral } \\
\text { NOX4 vs WT mice }\end{array}$ & FA ligation & 4 weeks & $\uparrow$ & $\uparrow$ & n.a & 67 \\
\hline Global NOX4 KO vs WT & FA ligation & & $\downarrow$ & n.a & $\downarrow$ & 114 \\
\hline Inducible NOX4 KO vs WT & & & $\downarrow$ & n.a & $\downarrow$ & \\
\hline
\end{tabular}


Table 2, continued

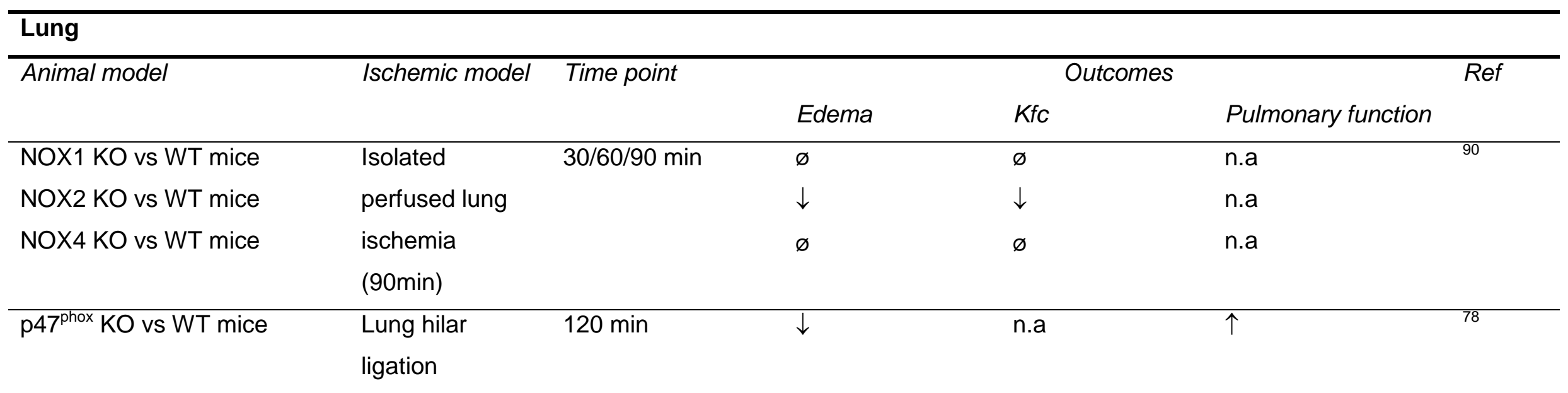

FA, femoral artery; LAD, left anterior descending coronary artery; pMCAO, permanent middle cerebral artery occlusion; tMCAO, transient

$\stackrel{\omega}{ }$ middle cerebral artery occlusion; ø, no change. 
Table 3. Overview of the in vitro studies showing a role of NAPDH oxidases in the process of ischemia-reperfusion injury.

\begin{tabular}{|c|c|c|c|}
\hline Cell/tissue & Treatment & Outcome & Ref \\
\hline \multicolumn{4}{|c|}{ ROS production and NOX expression } \\
\hline H9c2 cardiac cells & $\mathrm{I} / \mathrm{R}$ & $\uparrow \mathrm{ROS}, \uparrow \mathrm{p} 47^{\text {phox }}, \uparrow \mathrm{NOX} 2$ protein & 80 \\
\hline \multirow[t]{2}{*}{ H9c2 cardiac cells } & Metabolic inhibition (ischemia) & $\uparrow \mathrm{ROS}, \uparrow$ NOX2 mRNA, $\uparrow$ apoptosis & 81 \\
\hline & $\begin{array}{l}\text { Metabolic inhitbion + DPI or } \\
\text { apocynin }\end{array}$ & $\downarrow$ ROS increase, $\downarrow$ apoptosis & \\
\hline Human PASMC & Hypoxia & $\uparrow$ NOX4 mRNA and protein and HIF-1 $1 \alpha$ mRNA and protein & 51 \\
\hline HepG2 cells & Hypoxia and overexpression HIF-1 $\alpha$ & $\uparrow$ Cell proliferation and ROS production & \\
\hline HEK293 & $\begin{array}{l}\text { Hypoxia, overexpression HIF-1 } \alpha \text { and } \\
\text { depletion NOX4 with shRNA }\end{array}$ & $\downarrow$ of increased proliferation and ROS production & \\
\hline \multicolumn{4}{|l|}{ Angiogenesis } \\
\hline Human and bovine EC & Adenoviral overexpression or & $\uparrow$ Tube formation in overexpressing, $\downarrow$ tube formation in knockdown & 67 \\
\hline Aortas from EC-specific & knockdown NOX4 & cells & \\
\hline NOX4 Tg and WT mice & -- & $\uparrow$ Capillary sprouting in Tg aortas & \\
\hline
\end{tabular}


Table 3, continued

\begin{tabular}{|c|c|c|c|}
\hline Cell/tissue & Treatment & Outcome & $\operatorname{Ref}$ \\
\hline HMEC and HUVEC & siRNA to NOX4 & $\downarrow$ Tube formation and $\downarrow$ wound healing & 112 \\
\hline HMEC & Adenoviral transfection Nox4 & $\downarrow$ EC migration and proliferation, $\downarrow$ tube formation but $\uparrow$ wound healing & \\
\hline HCAEC/HUVEC & $\mathrm{p} 47^{\text {phox }}$ siRNA & $\downarrow$ VEGF mediated phosphorylation of VEGFR2 & 99 \\
\hline $\begin{array}{l}\text { Human endothelial cells } \\
\text { Mouse vena cava } \\
\text { explants }\end{array}$ & $\begin{array}{l}\text { Urotensin } \\
\text { Urotensin in Nox2 depleted cells } \\
\text { Urotensin }\end{array}$ & $\begin{array}{l}\uparrow \text { Formation capillary like structures in Matrigel } \\
\downarrow \text { Formation capillary like structures in Matrigel } \\
\uparrow \text { Sprouting in Matrigel }\end{array}$ & 73 \\
\hline \multicolumn{4}{|l|}{ Remodeling } \\
\hline $\begin{array}{l}\text { VSMC from aortas of } \\
\text { Sprague-Dawley rats }\end{array}$ & $\begin{array}{l}\text {-- } \\
\text { SiRNA and antisense adenovirus to } \\
\text { Nox4 }\end{array}$ & $\begin{array}{l}\text { High NOX4 mRNA in differentiating cells, low NOX4 in } \\
\text { dedifferentiating cells; Low NOX1 mRNA in differentiating cells, high } \\
\text { NOX1 in dedifferentiating cells } \\
\downarrow \text { Differentiation markers and } \downarrow \text { contractile type stress fibers }\end{array}$ & 91 \\
\hline $\begin{array}{l}\text { Aortic VSMC from NOX1 } \\
\text { Tg and WT mice }\end{array}$ & AT-II & $\begin{array}{l}\uparrow \text { ROS production in } \mathrm{Tg} \text {, blood pressure elevation in vivo higher in } \mathrm{Tg} \text {, } \\
\text { greater hypertrophy (aortic medial thickness) in } \mathrm{Tg}\end{array}$ & 109 \\
\hline $\begin{array}{l}\text { Mouse ESC } \\
\text { Neonatal mouse } \\
\text { cardiomyocytes }\end{array}$ & $\begin{array}{l}\text { NOX4 SiRNA, Nox4 ShRNA, NOX4 } \\
\text { riRNA }\end{array}$ & $\begin{array}{l}\downarrow \text { Spontaneously beating EB's, } \\
\text { Impaired cardiac myofibrillogenesis }\end{array}$ & 152 \\
\hline
\end{tabular}


Table 3, continued

\begin{tabular}{|c|c|c|c|}
\hline $\begin{array}{l}\text { Neonatal rat } \\
\text { cardiomyocytes } \\
\text { Cardiomyocytes from } \\
\text { mice }\end{array}$ & NOX4 overexpression & Cell size $\varnothing, \uparrow$ apoptosis in overexpressing cells & 118 \\
\hline Human PASMC & $\begin{array}{l}\text { Urotensin-II } \\
\text { Urotensin-II and antisense vectors } \\
\text { against } \mathrm{p} 22^{\text {phox }} \text { and NOX4 }\end{array}$ & $\begin{array}{l}\uparrow \text { p22 } 2^{\text {phox }} \text { and NOX4 protein expression, } \uparrow \text { ROS production } \\
\downarrow \text { of increased ROS production }\end{array}$ & 116 \\
\hline Human PASMC & $\begin{array}{l}\text { TGF- } \beta 1 \\
\text { TGF- } \beta 1 \text { and siRNA against NOX4 }\end{array}$ & $\begin{array}{l}\uparrow \text { NOX4 mRNA and protein expression and ROS production } \\
\downarrow \text { TGF- } \beta 1 \text { induced proliferation }\end{array}$ & 117 \\
\hline
\end{tabular}

AT-II, angiotensin II; DU145, epithelial cell line derived from human prostate tumor; EB, embryoid bodies; ESC, embryonic stem cells; HEK293, human embryonic kidney cells; HCAEC, human coronary artery endothelial cells; NIH 3T3 cells, mouse embryonic fibroblasts; HepG2, human hepatoblastoma cells; HMEC, human microvascular endothelial cells; SD rat, Sprague-Dawley rats; siRNA, short interference RNA; shRNA, short hairpin RNA; riRNA, ribosomal RNA; TAC, transient aortic constriction; VEGF, vascular endothelial growth factor; VSMC, vascular smooth muscle cells; ø, no change. 


\section{Other sources of ROS in IRI}

As already mentioned, other sources of ROS distinct from NADPH oxidases generate ROS under non-physiological conditions. This indicates that these sources might also play a role in IRI. For example, xanthine oxidoreductase (XOR) catalyses the formation of xanthine and uric acid from hypoxanthine. It can exist in two different forms, xanthine dehydrogenase that transfers electrons to $\mathrm{NAD}^{+}$and xanthine oxidase, which cannot bind $\mathrm{NAD}^{+}$thus transferring electrons directly to molecular oxygen ${ }^{120}$. Under conditions of inflammation or oxidative stress, xanthine dehydrogenase can be post-translationally modified to the ROS producing xanthine oxidase ${ }^{20,120}$. The evidence for a role of XOR in IRI is controversial showing both positive and negative roles as reviewed in ${ }^{16,20,120}$.

Nitric oxide synthase catalyses the formation of nitric oxide (NO) from L-arginine, using tetrahydrobiopterin $\left(\mathrm{BH}_{4}\right)$ as an essential, redox sensitive cofactor. NOS, too, can have double-edged roles within the vasculature: NO is beneficial by mediating, for example, vasorelaxation, anti-coagulant and anti-proliferative properties ${ }^{10}$. However during IRI, ROS uncouple NOS by oxidising $\mathrm{BH}_{4}{ }^{121}$. NOS itself then produces ROS instead of NO ${ }^{10,12,122}$. In addition, NO can react with superoxide to form toxic peroxynitrite ${ }^{123,124}$ causing further cell and tissue damage.

Mitochondria have also been described as being major contributors to ROS production in IRI, especially in the heart ${ }^{17,125-128}$. Mitochondria form ROS via electron leakage from the electron transport chain during oxidative phosphorylation at different complexes ${ }^{129}$ or via less common ways involving mono amino oxidases or p66Shc protein ${ }^{18}$. Similar as in case of NOS, ROS produced by mitochondria or by other sources can lead to a further increased production of ROS: ROS induced ROS release ${ }^{130,131}$. Mitochondrial ROS have been shown to also play a role in ischemic preconditioning ${ }^{132,133}$.

Interplay between different sources of ROS can also be one of the mechanisms contributing to IRI ${ }^{11,22,129}$. NOX4, for example, has been shown to localise in mitochondria, indicating that NOX4 might produce mitochondrial ROS ${ }^{40}$. 
The ROS produced by NADPH oxidases can then trigger mitochondrial dysfunction, leading to increased ROS production and, vice versa, ROS produced by mitochondria can activate NADPH oxidases. This has recently been reviewed in more detail elsewhere ${ }^{129}$.

Nevertheless, it has yet to be established which source(s) of ROS are the pathologically relevant ones in IRI. However, again NADPH oxidases are the only enzymes with the sole function of generating ROS. Therefore, targeting NADPH oxidases seems a new promising therapeutic strategy, which seems superior compared to using antioxidants, as is discussed below.

\section{Therapeutic strategies}

\section{Antioxidants}

As ROS and oxidative stress are likely major players in IRI, attempts to prevent and treat related disorders with antioxidant supplements seemed plausible. Indeed, many clinical trials testing the effects of antioxidant application have been performed.

Some, rather small clinical trials tested the acute or sub-acute therapeutic effects of antioxidants in patients with $\mathrm{I} / \mathrm{R}$ related disorders, with conflicting results. For example, in patients undergoing aortic cross-clamping, oral supplementation of vitamin E (60 mg for 8 days pre-surgery) prevented overproduction of ROS and diminished muscle damage in the lower limbs ${ }^{134}$. However, such a preventive intervention is by definition excluded in post-infarct or stroke therapies. Nevertheless, in patients with diabetes, but not in non-diabetic patients, a reduction in 30-day mortality after acute myocardial infarction was found when patients were treated with high doses of vitamin C (1000 mg IV in 12 hours, $400 \mathrm{mg}$ orally) and E (200 mg, 2 times daily) for 30 days ${ }^{6}$. In contrast, human superoxide dismutase (SOD) (IV bolus $10 \mathrm{mg} / \mathrm{kg}$ followed by infusion $0.2 \mathrm{mg} / \mathrm{kg} / \mathrm{min}$ for $60 \mathrm{~min}$.) did not show any effect after acute myocardial infarction in patients undergoing percutaneous transluminal coronary angioplasty (PTCA) ${ }^{135}$. In smokers with myocardial infarction, oral supplementation of vitamin $\mathrm{E}$ ( $50 \mathrm{mg}$ daily) did not reduce the incidence of recurrent myocardial infarction ${ }^{136}$. Alarmingly, administration of Vitamin C ( $2 \mathrm{~g}$ IV 2 hours prior to surgery) in patients undergoing elective IR (aortic clamping), promoted ironinduced oxidative lipid damage via a Fenton-type reaction. 
The subsequent release of lactate dehydrogenase (LDH) into the systemic circulation may have catalysed formation of second-generation radicals implicated in the regulation of vascular permeability and angiogenesis ${ }^{137}$. The reasons for the conflicting results are not known, but may be caused by the use of different antioxidants, doses and timing as well as patient collectives. Nevertheless, as these studies only included relatively small patient numbers they rather have a pilot character.

Many large and long trials tested antioxidant supplements for primary and secondary prevention of $\mathrm{I} / \mathrm{R}$ events. Despite the huge patient numbers, study results are conflicting $3,5,6,136,138,139$. However, several meta-analysis suggest that application of antioxidants does not improve cardiovascular mortality and morbidity. In contrast, untargeted supplementation of antioxidants may even be harmful ${ }^{5,27,29,30,140}$. Thus, although ROS play an important role in IRI, antioxidants failed to prevent related disorders. Several explanations for this failure have been proposed. For example, antioxidants given orally might not penetrate into the vascular wall; scavenging of radicals can convert antioxidants to radicals themselves exerting pro-oxidant effects; and the timing of the application might not be right, since ROS play a role already in the initiation of cardiovascular diseases ${ }^{27,140}$. Furthermore, antioxidants - if they reach the target tissue - cannot distinguish between good and bad ROS.

Thus, unravelling the role of ROS and their sources at different time points in IRI is crucial for designing the optimal therapeutic approach. Preventing the formation of disease-triggering ROS at the right time, instead of scavenging them after they have been formed, seems a better therapeutic approach.

\section{NADPH oxidase as therapeutic targets in IRI}

NADPH oxidases are crucial players in all phases of IRI. Thus, interfering with the NADPH oxidase function is a promising strategy for treating IRI. However, important issues need to be considered: First, timing is likely to be crucial, since NADPH oxidases and ROS play different roles depending on the time course of IRI. Second, isoform specific differences or organ/cell-specific effects have to be taken into account. 
Third, the amount of ROS is important, since low amounts of ROS are needed for physiologic functions such as regulation of cell-growth and differentiation ${ }^{11,12}$, whereas increased levels surpass endogenous anti-oxidant defence and cause cell damage.

The underlying mechanisms of all these factors are only incompletely understood. During ischemia, NADPH oxidases interact with HIF ${ }^{50-52,54-56}$ and may play a role in oxygen sensing 48,49,58. However, the involvement of individual NOX isoforms and whether inhibition or rather enhancing NADPH oxidase activity during this phase is the appropriate therapeutic approach is unclear.

During reperfusion, NADPH oxidase inhibition could be a new therapeutic option in acute events, such as stroke or myocardial infarction, if it is possible to intervene exactly during this period. Patients diagnosed with ischemic stroke or myocardial infarction could be treated with NADPH oxidase inhibitors even before measures to establish reperfusion are taken. Also, in patients in whom reperfusion has already occurred endogenously, treatment with an NADPH oxidase inhibitor could be initiated in the early phase of reperfusion. For example, in brain IRI, inhibition of NOX4 postreperfusion is beneficial in mice after an ischemic stroke ${ }^{63}$. NOX4 has been found in mitochondria ${ }^{40}$. As mitochondrial ROS likely have detrimental roles in the acute damaging phase after I/R of the heart ${ }^{125}$, reducing mitochondrial ROS generation by NOX4 inhibition is an attractive strategy. NOX2 may also be a good target in this condition, since an inflammatory reaction and disturbed blood brain barrier promotes influx of neutrophils into the ischemic brain area ${ }^{86}$. However, data regarding NOX2 are conflicting ${ }^{89}$. In heart IRI, even more NOX isoforms may play a role ${ }^{22}$. Importantly, NOX5, the only NOX isoform, which is activated by calcium, is a potential target to reduce oxidative stress in VSMC ${ }^{39,141}$ as well as in EC ${ }^{38}$ and possibly also directly in the heart. Unfortunately, due to the lack of NOX5 in rats and mice, no in vivo data on the role of NOX5 in animal models of MI are available.

During the third post-reperfusion phase, ROS appear to be needed for angiogenesis ${ }^{15}$. However, at the same time, they can be detrimental in promoting hypertrophy or dilatation. Here, the amount of ROS and the place where they are produced, but also the underlying causes of remodelling seem crucial, as is shown by divergent results in different pressure overload models ${ }^{142,143}$. 
In chronic ischemic diseases, such as peripheral artery disease (PAD), inhibition of NADPH oxidases could lead to an even more impaired blood flow ${ }^{115}$ via interfering with angiogenesis. Here, NADPH oxidase-stimulated angiogenesis could lead to a better preserved vascularisation and oxygen delivery within the peripheral tissues ${ }^{68}$. Thus, activation of specific NOX isoforms at specific locations may here be a therapeutic option for PAD patients. Since NOX1, NOX2 and NOX4 are expressed in both VSMCs and ECs ${ }^{22}$, they all seem to be possible targets in chronic ischemic disease states.

\section{NADPH oxidase inhibitors}

As is clear from the discussion in the previous chapter, complete inhibition of NADPH oxidase activities may not be a good strategy. Rather, it is important to delineate the precise roles of individual NOX isoforms, and it may turn out that one isoform should be inhibited, while another should be left untouched or even activated. Isoformspecific NOX inhibitors developed as therapeutic drugs could be used at specific time-points during IRI.

Due to the high degree of structural similarity among the NOX enzymes ${ }^{22}$, the development of isoform selective NADPH oxidase inhibitors seems difficult. However, to avoid unforeseeable and potentially severe side effects of unselective NOX inhibition, this is highly desired. Currently, there is only one NOX isoform-specific inhibitor published, which is the NOX2 inhibitory peptide gp91ds-tat ${ }^{144}$. However, peptides are not orally bioavailable and probably do not show suitable pharmacokinetic parameters for clinical applications. Thus, small molecule inhibitors are better suited. Nevertheless, published small molecule NADPH oxidase inhibitors with some degree of selectivity for one NOX isoform, such as ML171 ${ }^{145}$ and M090 ${ }^{146}$ (NOX1 selectivity), do not seem to have satisfying pharmacokinetic properties. Other inhibitors, both isoform selective and unselective, have not yet been thoroughly investigated for off-target effects and toxicity, for example fulvene-5, VAS2870, M090 and to some degree ML171 ${ }^{83,145-147}$. VAS2870 has been shown to reduce ROS production in vitro ${ }^{148}$ and to diminish brain damage after stroke in vivo ${ }^{63}$. 
However, initially, VAS2870 seemed to be specific for NADPH oxidases ${ }^{139}$, but recently potential off-target effects of VAS2870 have been reported ${ }^{149}$ with thiol alkylation as the likely mechanism of action of VAS2870-mediated NADPH oxidase inhibition. Clearly, the importance of this off-target alkylation or possible other offtarget effects of VAS2870 need further analysis. The first inhibitor showing suitable toxicity and high oral bioavailability was the NOX1 and NOX4 inhibitor GKT136901 ${ }^{150}$ that was further optimised to GKT137831. The latter compound is now in a phase-I clinical study for the indication diabetic nephropathy.

In summary, (isoform) specific NOX inhibitors or stimulators with a good pharmacodynamic and pharmacokinetic profile would be a significant step forward in the treatment of diseases, in which IRI plays a role.

\section{Summary and outlook}

Isoform selective inhibition of NADPH oxidases seems to be a promising strategy to treat stroke and myocardial infarction. Instead of scavenging ROS with antioxidants, selective blockade of ROS production at pathologically important sites may open new doors for therapies. Timing of selective NOX blockade may be of utmost importance, because of the opposing effects in the different phases of IRI. In the post-reperfusion phase, stimulation of NADPH oxidases might even be an option. The same may hold true for more chronic ischemic diseases, in which ROS likely mediate angiogenesis. Thus, it is clear that unravelling the precise sequence of events and differential roles of the NADPH oxidase-ROS axis is crucial for successful therapeutic clinical translation. Keeping in mind the double-edged sword that NADPH oxidases present, we must defeat the detrimental effects and possibly embrace the beneficial effects at the same time.

\section{ACKNOWLEDGMENTS}

The authors wish to acknowledge national and international competitive grant support by the NHMRC, EU and ERC (HHHWS). 


\section{AUTHOR DISCLOSURE STATEMENT}

HHHWS is a co-founder of Vasopharm GmbH, which has developed a NOX inhibitor, and also holds shares of Vasopharm $\mathrm{GmbH}$.

\section{References}

1 Eltzschig, H. K. \& Collard, C. D. Vascular ischaemia and reperfusion injury. Br Med Bull 70, 71-86 (2004).

2 Maxwell, S. R. \& Lip, G. Y. Reperfusion injury: a review of the pathophysiology, clinical manifestations and therapeutic options. Int J Cardiol 58, 95-117 (1997).

3 Vivekananthan, D. P., Penn, M. S., Sapp, S. K., Hsu, A. \& Topol, E. J. Use of antioxidant vitamins for the prevention of cardiovascular disease: metaanalysis of randomised trials. Lancet 361, 2017-2023 (2003).

4 Bath, P. M. et al. Effects of NXY-059 in experimental stroke: an individual animal meta-analysis. British journal of pharmacology 157, 1157-1171 (2009).

5 Andreadou, I., Iliodromitis, E. K., Farmakis, D. \& Kremastinos, D. T. To prevent, protect and save the ischemic heart: antioxidants revisited. Expert Opin Ther Targets 13, 945-956 (2009).

6 Jaxa-Chamiec, T., Bednarz, B., Herbaczynska-Cedro, K., Maciejewski, P. \& Ceremuzynski, L. Effects of vitamins $C$ and $E$ on the outcome after acute myocardial infarction in diabetics: a retrospective, hypothesis-generating analysis from the MIVIT study. Cardiology 112, 219-223 (2009).

7 Griendling, K. K., Sorescu, D. \& Ushio-Fukai, M. NAD(P)H oxidase: role in cardiovascular biology and disease. Circ Res 86, 494-501 (2000).

8 Gourdin, M. J., Bree, B. \& De Kock, M. The impact of ischaemia-reperfusion on the blood vessel. Eur J Anaesthesiol 26, 537-547, (2009).

9 Yellon, D. M. \& Hausenloy, D. J. Myocardial reperfusion injury. N Engl J Med 357, 1121-1135 (2007).

10 Forstermann, U. Oxidative stress in vascular disease: causes, defense mechanisms and potential therapies. Nat Clin Pract Cardiovasc Med 5, 338349(2008).

11 Elahi, M. M., Kong, Y. X. \& Matata, B. M. Oxidative stress as a mediator of cardiovascular disease. Oxid Med Cell Longev 2, 259-269 (2009). 
12 Griendling, K. K. \& FitzGerald, G. A. Oxidative stress and cardiovascular injury: Part I: basic mechanisms and in vivo monitoring of ROS. Circulation 108, 1912-1916 (2003).

13 Braunwald, E. \& Kloner, R. A. Myocardial reperfusion: a double-edged sword? J Clin Invest 76, 1713-1719 (1985).

14 Maulik, N. Redox regulation of vascular angiogenesis. Antioxid Redox Signal 4, 783-784 (2002).

15 Maulik, N. \& Das, D. K. Redox signaling in vascular angiogenesis. Free Radic Biol Med 33, 1047-1060 (2002).

16 Berry, C. E. \& Hare, J. M. Xanthine oxidoreductase and cardiovascular disease: molecular mechanisms and pathophysiological implications. J Physiol 555, 589-606 (2004).

17 Di Lisa, F. \& Bernardi, P. Mitochondria and ischemia-reperfusion injury of the heart: fixing a hole. Cardiovasc Res 70, 191-199 (2006).

18 Di Lisa, F., Canton, M., Menabo, R., Kaludercic, N. \& Bernardi, P. Mitochondria and cardioprotection. Heart Fail Rev 12, 249-260 (2007).

19 Gao, L., Laude, K. \& Cai, H. Mitochondrial pathophysiology, reactive oxygen species, and cardiovascular diseases. Vet Clin North Am Small Anim Pract 38, 137-155, vi (2008).

20 Harrison, R. Structure and function of xanthine oxidoreductase: where are we now? Free Radic Biol Med 33, 774-797 (2002).

21 Meneshian, A. \& Bulkley, G. B. The physiology of endothelial xanthine oxidase: from urate catabolism to reperfusion injury to inflammatory signal transduction. Microcirculation 9, 161-175 (2002).

22 Cave, A. C. et al. NADPH oxidases in cardiovascular health and disease. Antioxid Redox Signal 8, 691-728 (2006).

23 Lambeth, J. D. NOX enzymes and the biology of reactive oxygen. Nat Rev Immunol 4, 181-189 (2004).

24 Lassegue, B. \& Griendling, K. K. NADPH oxidases: functions and pathologies in the vasculature. Arterioscler Thromb Vasc Biol 30, 653-661 (2010).

25 Sirker, A., Zhang, M. \& Shah, A. M. NADPH oxidases in cardiovascular disease: insights from in vivo models and clinical studies. Basic Res Cardiol 106 (5), 735-747 (2011). 
26 Rivera, J., Sobey, C. G., Walduck, A. K. \& Drummond, G. R. Nox isoforms in vascular pathophysiology: insights from transgenic and knockout mouse models. Redox Rep 15, 50-63 (2010).

27 Guzik, T. J. \& Harrison, D. G. Vascular NADPH oxidases as drug targets for novel antioxidant strategies. Drug Discov Today 11, 524-533 (2006).

28 Bedard, K. \& Krause, K. H. The NOX family of ROS-generating NADPH oxidases: physiology and pathophysiology. Physiol Rev 87, 245-313 (2007).

29 Hamilton, C. A. et al. Strategies to reduce oxidative stress in cardiovascular disease. Clin Sci (Lond) 106, 219-234 (2004).

30 Williams, H. C. \& Griendling, K. K. NADPH oxidase inhibitors: new antihypertensive agents? J Cardiovasc Pharmacol 50, 9-16 (2007).

31 Geiszt, M. NADPH oxidases: new kids on the block. Cardiovasc Res 71, 289299 (2006).

32 Lassegue, B. \& Clempus, R. E. Vascular NAD(P)H oxidases: specific features, expression, and regulation. Am J Physiol Regul Integr Comp Physiol 285, R277-297 (2003).

33 Gorlach, A. et al. A gp91phox containing NADPH oxidase selectively expressed in endothelial cells is a major source of oxygen radical generation in the arterial wall. Circ Res 87, 26-32 (2000).

34 Jones, S. A. et al. Expression of phagocyte NADPH oxidase components in human endothelial cells. The American journal of physiology 271, H1626-1634 (1996).

35 Wingler, $\mathrm{K}$. et al. Upregulation of the vascular $\mathrm{NAD}(\mathrm{P}) \mathrm{H}$-oxidase isoforms Nox1 and Nox4 by the renin-angiotensin system in vitro and in vivo. Free radical biology \& medicine 31, 1456-1464 (2001).

36 Miller, A. A., Drummond, G. R., Schmidt, H. H. \& Sobey, C. G. NADPH oxidase activity and function are profoundly greater in cerebral versus systemic arteries. Circulation research 97, 1055-1062 (2005).

37 Miller, A. A., Drummond, G. R., Mast, A. E., Schmidt, H. H. \& Sobey, C. G. Effect of gender on NADPH-oxidase activity, expression, and function in the cerebral circulation: role of estrogen. Stroke; a journal of cerebral circulation $38,2142-2149$ (2007). 
38 BelAiba, R. S. et al. NOX5 variants are functionally active in endothelial cells. Free Radic Biol Med 42, 446-459 (2007).

39 Jay, D. B. et al. Nox5 mediates PDGF-induced proliferation in human aortic smooth muscle cells. Free Radic Biol Med 45, 329-335 (2008).

40 Block, K., Gorin, Y. \& Abboud, H. E. Subcellular localization of Nox4 and regulation in diabetes. Proc Natl Acad Sci U S A 106, 14385-14390 (2009).

41 Hilenski, L. L., Clempus, R. E., Quinn, M. T., Lambeth, J. D. \& Griendling, K. K. Distinct subcellular localizations of Nox1 and Nox4 in vascular smooth muscle cells. Arterioscler Thromb Vasc Biol 24, 677-683 (2004).

42 Lobysheva, I. et al. Moderate caveolin-1 downregulation prevents NADPH oxidase-dependent endothelial nitric oxide synthase uncoupling by angiotensin II in endothelial cells. Arteriosclerosis, thrombosis, and vascular biology 31, 2098-2105 (2011).

43 Ambasta, R. K. et al. Direct interaction of the novel Nox proteins with p22phox is required for the formation of a functionally active NADPH oxidase. The Journal of biological chemistry 279, 45935-45941 (2004).

44 Opitz, N., Drummond, G. R., Selemidis, S., Meurer, S. \& Schmidt, H. H. The 'A's and 'O's of NADPH oxidase regulation: a commentary on "Subcellular localization and function of alternatively spliced Noxo1 isoforms". Free Radic Biol Med 42, 175-179 (2007).

45 Lyle, A. N. et al. Poldip2, a novel regulator of Nox4 and cytoskeletal integrity in vascular smooth muscle cells. Circ Res 105, 249-259 (2009).

46 Brandes, R. P. et al. The vascular NADPH oxidase subunit p47phox is involved in redox-mediated gene expression. Free Radic Biol Med 32, 11161122 (2002).

47 Djordjevic, T. et al. The expression of the NADPH oxidase subunit p22phox is regulated by a redox-sensitive pathway in endothelial cells. Free Radic Biol Med 38, 616-630 (2005).

$48 \mathrm{He}$, L. et al. Characteristics of carotid body chemosensitivity in NADPH oxidase-deficient mice. Am J Physiol Cell Physiol 282, C27-33 (2002).

49 Jones, R. D., Hancock, J. T. \& Morice, A. H. NADPH oxidase: a universal oxygen sensor? Free Radic Biol Med 29, 416-424 (2000). 
50 Block, K. et al. NAD(P)H oxidases regulate HIF-2alpha protein expression. J Biol Chem 282, 8019-8026 (2007).

51 Diebold, I. et al. The hypoxia-inducible factor-2alpha is stabilized by oxidative stress involving NOX4. Antioxid Redox Signal 13, 425-436 (2010).

52 Diebold, I., Petry, A., Hess, J. \& Gorlach, A. The NADPH oxidase subunit NOX4 is a new target gene of the hypoxia-inducible factor-1. Mol Biol Cell 21, 2087-2096 (2010).

53 Gorlach, A. et al. Thrombin activates the hypoxia-inducible factor-1 signaling pathway in vascular smooth muscle cells: Role of the p22(phox)-containing NADPH oxidase. Circ Res 89, 47-54 (2001).

54 Goyal, $\mathrm{P}$. et al. Upregulation of $\mathrm{NAD}(\mathrm{P}) \mathrm{H}$ oxidase 1 in hypoxia activates hypoxia-inducible factor 1 via increase in reactive oxygen species. Free Radic Biol Med 36, 1279-1288 (2004).

55 Yuan, G. et al. Hypoxia-inducible factor 1 mediates increased expression of NADPH oxidase-2 in response to intermittent hypoxia. J Cell Physiol, 217 674685 (2011).

56 Yuan, G., Nanduri, J., Khan, S., Semenza, G. L. \& Prabhakar, N. R. Induction of HIF-1alpha expression by intermittent hypoxia: involvement of NADPH oxidase, Ca2+ signaling, prolyl hydroxylases, and mTOR. J Cell Physiol 217, 674-685 (2008).

57 Archer, S. L. et al. O2 sensing is preserved in mice lacking the gp91 phox subunit of NADPH oxidase. Proc Natl Acad Sci U S A 96, 7944-7949 (1999).

58 He, L. et al. Effect of p47phox gene deletion on ROS production and oxygen sensing in mouse carotid body chemoreceptor cells. Am J Physiol Lung Cell Mol Physiol 289, L916-924 (2005).

59 Roy, A. et al. Mice lacking in gp91 phox subunit of $\mathrm{NAD}(\mathrm{P}) \mathrm{H}$ oxidase showed glomus cell $[\mathrm{Ca}(2+)](\mathrm{i})$ and respiratory responses to hypoxia. Brain Res 872 , 188-193 (2000).

60 Mittal, M. et al. Hypoxia-dependent regulation of nonphagocytic NADPH oxidase subunit NOX4 in the pulmonary vasculature. Circ Res 101, 258-267 (2007). 
61 Rupin, A. et al. Role of NADPH oxidase-mediated superoxide production in the regulation of E-selectin expression by endothelial cells subjected to anoxia/reoxygenation. Cardiovasc Res 63, 323-330 (2004).

62 Hong, H., Zeng, J. S., Kreulen, D. L., Kaufman, D. I. \& Chen, A. F. Atorvastatin protects against cerebral infarction via inhibition of $\mathrm{NADPH}$ oxidase-derived superoxide in ischemic stroke. Am J Physiol Heart Circ Physiol 291, H2210-2215 (2006).

63 Kleinschnitz, C. et al. Post-stroke inhibition of induced NADPH oxidase type 4 prevents oxidative stress and neurodegeneration. PLoS Biol 8, (2010).

64 Vallet, P. et al. Neuronal expression of the NADPH oxidase NOX4, and its regulation in mouse experimental brain ischemia. Neuroscience 132, 233-238 (2005).

65 Doerries, C. et al. Critical role of the $\mathrm{NAD}(\mathrm{P}) \mathrm{H}$ oxidase subunit p47phox for left ventricular remodeling/dysfunction and survival after myocardial infarction. Circ Res 100, 894-903 (2007).

66 Looi, Y. H. et al. Involvement of Nox2 NADPH oxidase in adverse cardiac remodeling after myocardial infarction. Hypertension 51, 319-325 (2008).

67 Zhao, W., Zhao, D., Yan, R. \& Sun, Y. Cardiac oxidative stress and remodeling following infarction: role of NADPH oxidase. Cardiovasc Pathol 18, 156-166 (2009).

68 Craige, S. M. et al. NADPH oxidase 4 promotes endothelial angiogenesis through endothelial nitric oxide synthase activation. Circulation 124, 731-740 (2011).

69 Kietzmann, T. \& Gorlach, A. Reactive oxygen species in the control of hypoxia-inducible factor-mediated gene expression. Semin Cell Dev Biol 16, 474-486 (2005).

70 Semenza, G. L. Hypoxia-inducible factor 1 (HIF-1) pathway. Sci STKE 2007, (2007).

71 Dery, M. A., Michaud, M. D. \& Richard, D. E. Hypoxia-inducible factor 1: regulation by hypoxic and non-hypoxic activators. Int J Biochem Cell Biol 37, 535-540 (2005). 
72 Bonello, S. et al. Reactive oxygen species activate the HIF-1alpha promoter via a functional NFkappaB site. Arterioscler Thromb Vasc Biol 27, 755-761 (2007).

73 Diebold, I., Djordjevic, T., Hess, J. \& Gorlach, A. Rac-1 promotes pulmonary artery smooth muscle cell proliferation by upregulation of plasminogen activator inhibitor-1: role of NFkappaB-dependent hypoxia-inducible factor1alpha transcription. Thrombosis and haemostasis 100, 1021-1028 (2008).

74 Diebold I, P. A., Sabrane K, Djordjevic T, Hess J, Görlach. . The HIF1 target gene NOX2 promotes angiogenesis through urotensin-II. J Cell Sci In press.

75 Gorlach, A. et al. Reactive oxygen species modulate HIF-1 mediated PAI-1 expression: involvement of the GTPase Rac1. Thromb Haemost 89, 926-935 (2003).

76 Hirota, K. \& Semenza, G. L. Rac1 activity is required for the activation of hypoxia-inducible factor 1 . The Journal of biological chemistry $276,21166-$ 21172 (2001).

77 Chen, H., Song, Y. S. \& Chan, P. H. Inhibition of NADPH oxidase is neuroprotective after ischemia-reperfusion. J Cereb Blood Flow Metab 29, 1262-1272 (2009).

78 Chen, H., Kim, G. S., Okami, N., Narasimhan, P. \& Chan, P. H. NADPH oxidase is involved in post-ischemic brain inflammation. Neurobiol Dis 42, 341-348 (2011).

79 Yang, Z., Sharma, A. K., Marshall, M., Kron, I. L. \& Laubach, V. E. NADPH oxidase in bone marrow-derived cells mediates pulmonary ischemiareperfusion injury. Am J Respir Cell Mol Biol 40, 375-381 (2009).

80 Heumuller, S. et al. Apocynin is not an inhibitor of vascular NADPH oxidases but an antioxidant. Hypertension 51, 211-217 (2008).

81 Borchi, E. et al. Role of NADPH oxidase in H9c2 cardiac muscle cells exposed to simulated ischaemia-reperfusion. J Cell Mol Med 13, 2724-2735 (2009).

82 Meischl, C. et al. Ischemia induces nuclear NOX2 expression in cardiomyocytes and subsequently activates apoptosis. Apoptosis 11, 913-921 (2006).

83 Wind, S. et al. Comparative pharmacology of chemically distinct NADPH oxidase inhibitors. Br J Pharmacol 161, 885-898 (2010). 
84 Jackman, K. A., Miller, A. A., Drummond, G. R. \& Sobey, C. G. Importance of NOX1 for angiotensin II-induced cerebrovascular superoxide production and cortical infarct volume following ischemic stroke. Brain Res 1286, 215-220 (2009).

85 Kahles, T. et al. NADPH oxidase Nox1 contributes to ischemic injury in experimental stroke in mice. Neurobiology of disease 40, 185-192 (2010).

86 Brait, V. H. et al. Mechanisms contributing to cerebral infarct size after stroke: gender, reperfusion, T lymphocytes, and Nox2-derived superoxide. J Cereb Blood Flow Metab 30, 1306-1317 (2010).

87 Kahles, T. et al. NADPH oxidase plays a central role in blood-brain barrier damage in experimental stroke. Stroke 38, 3000-3006 (2007).

88 Walder, C. E. et al. Ischemic stroke injury is reduced in mice lacking a functional NADPH oxidase. Stroke 28, 2252-2258 (1997).

89 Radermacher, K. A. et al. The 1027th target candidate in stroke: Will NADPH oxidase hold up? Exp Transl Stroke Med 4, 11 (2012).

90 Duilio, C. et al. Neutrophils are primary source of $\mathrm{O} 2$ radicals during reperfusion after prolonged myocardial ischemia. Am J Physiol Heart Circ Physiol 280, H2649-2657 (2001).

91 Weissmann, N. et al. Activation of TRPC6 channels is essential for lung ischaemia-reperfusion induced oedema in mice. Nat Commun 3, 649 (2012).

92 Clempus, R. E. et al. Nox4 is required for maintenance of the differentiated vascular smooth muscle cell phenotype. Arterioscler Thromb Vasc Biol 27, 4248 (2007).

93 Cucoranu, I. et al. NAD(P)H oxidase 4 mediates transforming growth factorbeta1-induced differentiation of cardiac fibroblasts into myofibroblasts. Circ Res 97, 900-907 (2005).

94 Deliri, H. \& McNamara, C. A. Nox 4 regulation of vascular smooth muscle cell differentiation marker gene expression. Arterioscler Thromb Vasc Biol 27, 1214 (2007).

95 Frey, R. S., Ushio-Fukai, M. \& Malik, A. B. NADPH oxidase-dependent signaling in endothelial cells: role in physiology and pathophysiology. Antioxid Redox Signal 11, 791-810 (2009). 
96 Li, J. M. \& Shah, A. M. Endothelial cell superoxide generation: regulation and relevance for cardiovascular pathophysiology. Am J Physiol Regul Integr Comp Physiol 287, R1014-1030 (2004).

97 Ushio-Fukai, M. Redox signaling in angiogenesis: role of NADPH oxidase. Cardiovasc Res 71, 226-235 (2006).

98 Ushio-Fukai, M. \& Alexander, R. W. Reactive oxygen species as mediators of angiogenesis signaling: role of $\mathrm{NAD}(\mathrm{P}) \mathrm{H}$ oxidase. Mol Cell Biochem 264, 8597 (2004).

99 Ushio-Fukai, M. et al. Novel role of gp91(phox)-containing NAD(P)H oxidase in vascular endothelial growth factor-induced signaling and angiogenesis. Circ Res 91, 1160-1167 (2002).

100 Abid, M. R., Spokes, K. C., Shih, S. C. \& Aird, W. C. NADPH oxidase activity selectively modulates vascular endothelial growth factor signaling pathways. J Biol Chem 282, 35373-35385 (2007).

101 Urao, N. et al. Role of nox2-based NADPH oxidase in bone marrow and progenitor cell function involved in neovascularization induced by hindlimb ischemia. Circ Res 103, 212-220 (2008).

102 Tojo, T. et al. Role of gp91phox (Nox2)-containing NAD(P)H oxidase in angiogenesis in response to hindlimb ischemia. Circulation 111, 2347-2355 (2005).

103 Distasi, M. R. et al. Suppressed hindlimb perfusion in Rac2-/- and Nox2-/mice does not result from impaired collateral growth. Am J Physiol Heart Circ Physiol 296, H877-886 (2009).

104 Haddad, P. et al. Nox2-containing NADPH oxidase deficiency confers protection from hindlimb ischemia in conditions of increased oxidative stress. Arterioscler Thromb Vasc Biol 29, 1522-1528 (2009).

105 Ebrahimian, T. G. et al. NADPH oxidase-derived overproduction of reactive oxygen species impairs postischemic neovascularization in mice with type 1 diabetes. Am J Pathol 169, 719-728 (2006).

106 Frantz, S. et al. Left ventricular remodeling after myocardial infarction in mice with targeted deletion of the NADPH oxidase subunit gp91PHOX. Basic Res Cardiol 101, 127-132 (2006). 
107 van den Borne, S. W. et al. Mouse strain determines the outcome of wound healing after myocardial infarction. Cardiovasc Res 84, 273-282 (2009).

108 Hoffmeyer, M. R. et al. Myocardial ischemia/reperfusion injury in NADPH oxidase-deficient mice. Circ Res 87, 812-817 (2000).

109 Arbiser, J. L. et al. Reactive oxygen generated by Nox1 triggers the angiogenic switch. Proc Natl Acad Sci U S A 99, 715-720 (2002).

110 Dikalova, A. et al. Nox1 overexpression potentiates angiotensin II-induced hypertension and vascular smooth muscle hypertrophy in transgenic mice. Circulation 112, 2668-2676 (2005).

111 Arnold, R. S. et al. Hydrogen peroxide mediates the cell growth and transformation caused by the mitogenic oxidase Nox1. Proc Natl Acad Sci U S A 98, 5550-5555 (2001).

112 Suh, Y. A. et al. Cell transformation by the superoxide-generating oxidase Mox1. Nature 401, 79-82 (1999).

113 Datla, S. R. et al. Important role of Nox4 type NADPH oxidase in angiogenic responses in human microvascular endothelial cells in vitro. Arterioscler Thromb Vasc Biol 27, 2319-2324 (2007).

114 Schroder, K., Wandzioch, K., Helmcke, I. \& Brandes, R. P. Nox4 acts as a switch between differentiation and proliferation in preadipocytes. Arteriosclerosis, thrombosis, and vascular biology 29, 239-245 (2009).

115 Schroder, K. et al. Nox4 is a protective reactive oxygen species generating vascular NADPH oxidase. Circulation research 110, 1217-1225 (2012).

116 Maejima, Y., Kuroda, J., Matsushima, S., Ago, T. \& Sadoshima, J. Regulation of myocardial growth and death by NADPH oxidase. J Mol Cell Cardiol 50, 408-416 (2011).

117 Djordjevic, T. et al. Human urotensin II is a novel activator of NADPH oxidase in human pulmonary artery smooth muscle cells. Arteriosclerosis, thrombosis, and vascular biology 25, 519-525 (2005).

118 Sturrock, A. et al. Transforming growth factor-beta1 induces Nox4 NAD(P)H oxidase and reactive oxygen species-dependent proliferation in human pulmonary artery smooth muscle cells. Am J Physiol Lung Cell Mol Physiol 290, L661-L673 (2006). 
119 Ago, T. et al. Upregulation of Nox4 by hypertrophic stimuli promotes apoptosis and mitochondrial dysfunction in cardiac myocytes. Circ Res 106, 1253-1264 (2010).

120 Boueiz, A., Damarla, M. \& Hassoun, P. M. Xanthine oxidoreductase in respiratory and cardiovascular disorders. Am J Physiol Lung Cell Mol Physiol 294, L830-840 (2008).

121 Armitage, M. E., Wingler, K., Schmidt, H. H. \& La, M. Translating the oxidative stress hypothesis into the clinic: NOX versus NOS. J Mol Med 87, 1071-1076 (2009).

122 Otani, $\mathrm{H}$. The role of nitric oxide in myocardial repair and remodeling. Antioxid Redox Signal 11, 1913-1928 (2009).

123 Cai, H. \& Harrison, D. G. Endothelial dysfunction in cardiovascular diseases: the role of oxidant stress. Circ Res 87, 840-844 (2000).

124 Otani, H. Ischemic preconditioning: from molecular mechanisms to therapeutic opportunities. Antioxid Redox Signal 10, 207-247 (2008).

125 Chen, Q., Camara, A. K., Stowe, D. F., Hoppel, C. L. \& Lesnefsky, E. J. Modulation of electron transport protects cardiac mitochondria and decreases myocardial injury during ischemia and reperfusion. Am J Physiol Cell Physiol 292, C137-147 (2007).

126 Choi, K., Kim, J., Kim, G. W. \& Choi, C. Oxidative stress-induced necrotic cell death via mitochondira-dependent burst of reactive oxygen species. Curr Neurovasc Res 6, 213-222 (2009).

127 Loor, G. et al. Mitochondrial oxidant stress triggers cell death in simulated ischemia-reperfusion. Biochim Biophys Acta 1813, 1382-1394 (2011).

128 Perrelli, M. G., Pagliaro, P. \& Penna, C. Ischemia/reperfusion injury and cardioprotective mechanisms: Role of mitochondria and reactive oxygen species. World J Cardiol 3, 186-200 (2011).

129 Dikalov, S. Cross talk between mitochondria and NADPH oxidases. Free radical biology \& medicine 51, 1289-1301 (2011).

130 Gustafsson, A. B. \& Gottlieb, R. A. Heart mitochondria: gates of life and death. Cardiovasc Res 77, 334-343 (2008).

131 Zorov, D. B., Juhaszova, M. \& Sollott, S. J. Mitochondrial ROS-induced ROS release: an update and review. Biochim Biophys Acta 1757, 509-517 (2006). 
132 Kimura, S. et al. Role of $\mathrm{NAD}(\mathrm{P}) \mathrm{H}$ oxidase- and mitochondria-derived reactive oxygen species in cardioprotection of ischemic reperfusion injury by angiotensin II. Hypertension 45, 860-866 (2005).

133 Matsuzaki, S., Szweda, P. A., Szweda, L. I. \& Humphries, K. M. Regulated production of free radicals by the mitochondrial electron transport chain: Cardiac ischemic preconditioning. Adv Drug Deliv Rev 61, 1324-1331 (2009).

134 Novelli, G. P. et al. Vitamin E protects human skeletal muscle from damage during surgical ischemia-reperfusion. Am J Surg 173, 206-209 (1997).

135 Flaherty, J. T. et al. Recombinant human superoxide dismutase (h-SOD) fails to improve recovery of ventricular function in patients undergoing coronary angioplasty for acute myocardial infarction. Circulation 89, 1982-1991 (1994).

136 Rapola, J. M. et al. Randomised trial of alpha-tocopherol and beta-carotene supplements on incidence of major coronary events in men with previous myocardial infarction. Lancet 349, 1715-1720 (1997).

137 Bailey, D. M. et al. Vitamin C prophylaxis promotes oxidative lipid damage during surgical ischemia-reperfusion. Free Radical Biology and Medicine 40, 591-600 (2006).

138 Bjelakovic, G., Nikolova, D., Gluud, L. L., Simonetti, R. G. \& Gluud, C. Mortality in randomized trials of antioxidant supplements for primary and secondary prevention: systematic review and meta-analysis. Jama 297, 842857 (2007).

139 Wingler, K. et al. NOX 1, 2, 4, 5: Counting out oxidative stress. British Journal of Pharmacology Epub ahead of print (2011).

140 Griendling, K. K. \& FitzGerald, G. A. Oxidative stress and cardiovascular injury: Part II: animal and human studies. Circulation 108, 2034-2040 (2003).

141 Guzik, T. J. et al. Calcium-dependent NOX5 nicotinamide adenine dinucleotide phosphate oxidase contributes to vascular oxidative stress in human coronary artery disease. J Am Coll Cardiol 52, 1803-1809 (2008).

142 Kuroda, J. et al. NADPH oxidase 4 (Nox4) is a major source of oxidative stress in the failing heart. Proc Natl Acad Sci U S A 107, 15565-15570 (2010).

143 Zhang, M. et al. NADPH oxidase-4 mediates protection against chronic loadinduced stress in mouse hearts by enhancing angiogenesis. Proc Natl Acad Sci U S A 107, 18121-18126 (2010). 
144 Rey, F. E., Cifuentes, M. E., Kiarash, A., Quinn, M. T. \& Pagano, P. J. Novel competitive inhibitor of $\mathrm{NAD}(\mathrm{P}) \mathrm{H}$ oxidase assembly attenuates vascular $\mathrm{O}(2)$ (-) and systolic blood pressure in mice. Circ Res 89, 408-414 (2001).

145 Gianni, D. et al. A novel and specific NADPH oxidase-1 (Nox1) small-molecule inhibitor blocks the formation of functional invadopodia in human colon cancer cells. ACS Chem Biol 5, 981-993 (2010).

146 Brown, S. J. et al. in Probe Report for NOX1 inhibitors in Probe reports from the Molecular Libraries Program (2010).

147 Bhandarkar, S. S. Fulvene-5 potently inhibits NADPH oxidase 4 and blocks the growth of endothelial tumors in mice. The Journal of Clinical Investigation 0, 0-0 (2009).

148 Stielow, C. et al. Novel Nox inhibitor of oxLDL-induced reactive oxygen species formation in human endothelial cells. Biochemical and biophysical research communications 344, 200-205 (2006).

149 Sun, Q. A., Hess, D. T., Wang, B., Miyagi, M. \& Stamler, J. S. Off-target thiol alkylation by the NADPH oxidase inhibitor 3-benzyl-7-(2-benzoxazolyl)thio1,2,3-triazolo[4,5-d]pyrimidine (VAS2870). Free radical biology \& medicine 52, 1897-1902 (2012).

150 Laleu, B. et al. First in class, potent, and orally bioavailable NADPH oxidase isoform 4 (Nox4) inhibitors for the treatment of idiopathic pulmonary fibrosis. $\mathrm{J}$ Med Chem 53, 7715-7730 (2010).

151 Kunz, A. et al. Cyclooxygenase-2 does not contribute to postischemic production of reactive oxygen species. J Cereb Blood Flow Metab 27 545-551 (2007).

$152 \mathrm{Li}$, J. et al. The NADPH oxidase 4 drives cardiac differentiation: Role in regulating cardiac transcription factors and MAP kinase activation. Mol Biol Cell 17 3978-3988 (2006). 


\section{Chapter 3}

Identification of endothelial cells as a major vascular source of

NADPH oxidase 4-derived ROS causing blood brain barrier disruption, inflammation and neuronal damage in ischemic stroke

To examine which cell type is the source of the detrimental ROS formed by NOX4 in stroke, cell-specific NOX4 KO mice were generated and subjected to ischemic stroke. 


\begin{abstract}
Ischemic stroke is a major cause of morbidity and mortality in Western society. NADPH oxidase 4 has been identified as major source of reactive oxygen species (ROS) in stroke. A key event in the reperfusion phase of stroke is the breakdown of the blood-brain barrier leading to excitotoxicity and an inflammatory response and eventually neurodegeneration. NOX4 is expressed in several vascular and nonvascular cell types in the brain. Mechanistically and therapeutically it is important to know the cell type responsible for the damage to be able to specifically target NOX4 to reduce oxidative stress. Here we created mice with a cell specific genetic deletion of NOX4 in endothelial or smooth muscle cells. Upon tMCAO, endothelial cellspecific, but not vascular smooth muscle cell specific NOX4 KO mice showed similar neuroprotection as a global NOX4 knockout. Infarct sizes were smaller, inflammation reduced and the blood brain barrier stayed intact with less neuronal damage and, importantly, also improved neuronal function. In addition, using the NOX inhibitors VAS2870 and GKT 136901, we could reduce cell death after oxygen glucose deprivation in hippocampal brain slices. Thus, we here identify endothelial NOX4 as the major player in oxidative stress in stroke and easily accessible therapeutic target. Neuronal NOX4 might play an additional role, but in vivo data need to confirm this involvement.
\end{abstract}




\section{Introduction}

Ischemia reperfusion injury (IRI) causes tissue damage both by oxygen depletion in the ischemic phase and the formation of reactive oxygen species in the reperfusion phase. NADPH oxidases are major sources of ROS in the cardiovascular system and also in IRI. In stroke, the isoform NOX4, has been shown to play a detrimental role causing blood brain barrier disruption, inflammation, and neuronal damage ${ }^{1}$. Inhibiting NOX4 by either genetic knockout or pharmacological treatment reduced infarct size and resulted in a better neurological outcome, making NOX4 a possible innovative target for the treatment of ischemic stroke. However, ROS are also needed for physiological functioning of cells and tissues and can exert beneficial effects, depending on the amount produced, the cellular localisation and the timing ${ }^{2-4}$. Unspecific scavenging of ROS by antioxidants has been proven not to work to prevent damage in different forms of IRI ${ }^{5-8}$. Thus preventing the formation of ROS by inhibiting NOX, as the only enzymes solely dedicated to ROS production, seems to be a more promising approach. NOX4 is expressed in different cell types of the cardiovascular system (endothelial cells, vascular smooth muscle cells) as well as in neurons. Targeting specifically the most important cellular source of NOX4 might be even more attractive, since it leaves the beneficial roles of ROS intact. In this study, we created endothelial (EC) and vascular smooth muscle cell (VSMC)specific NOX4 KO mice and subjected them to ischemic stroke to investigate the cellspecific roles in ischemia reperfusion injury of the brain. In addition we used in vitro experiments assessing the possible role of neuronal NOX4 in ischemic brain damage.

\section{Results}

\section{Characterisation of the EC and VSMC-specific NOX4 KO mice}

Validation at the mRNA level

RT PCR on isolated endothelial cells from WT and eNOX4 KO mice showed the absence of NOX4 mRNA in the eNOX4 KO mice (Fig. 1A). Isolated vascular smooth muscle cells from sNOX4 KO mice showed a decreased NOX4 mRNA with RT-PCR. Subsequent qPCR showed 20\% mRNA left in the sNOX4 KO mice (Fig. 1B). 
This was expected, since the smooth muscle cell culture showed a contamination with some fibroblasts.
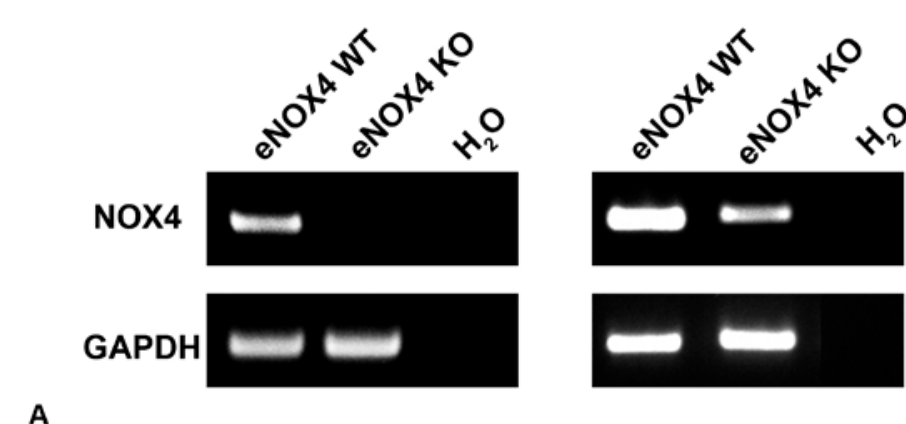

\section{A}

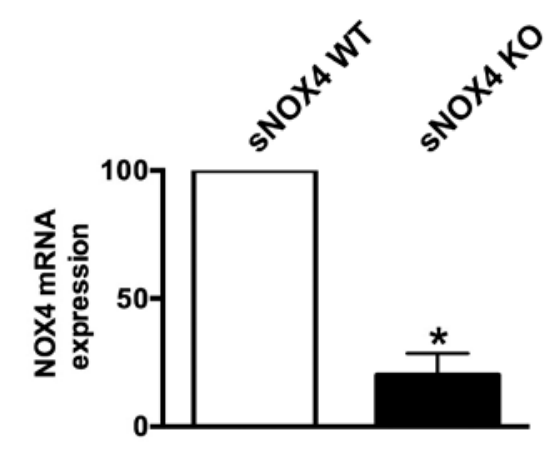

Figure 1. Validation of the endothelial and smooth muscle cell specific NOX4 KO mice at mRNA level. A) No NOX4 mRNA could be detected using RT-PCR in the eNOX4 KO mice Isolated smooth muscle cells of sNOX4 KO mice showed decreased NOX4 mRNA levels. B) Decreased mRNA expression (mean $\pm S E M, n=2$ ) in isolated vascular smooth muscle cells of sNOX4 KO (black bars) versus sNOX4 WT (white bars) as detected by qPCR. Expression levels were normalised to the $18 \mathrm{~S}$ gene and the expression in the WT was set to $100 \%$. The residual level of NOX4 mRNA in the isolated smooth muscle cells could be explained by a small contamination of fibroblasts in the cell culture.

RNA-scope also showed a decreased NOX4 mRNA in the media layer of sNOX4 KO mice compared to WT mice (Fig. 2).

\section{Vessel anatomy}

Fig. 3 shows the blood vessel anatomy at the basis of the brain, including the circle of Willis in WT and endothelial and smooth muscle cell specific NOX4 KO mice. No differences were seen in vessel distribution and all mice show a complete circle of Willis. 


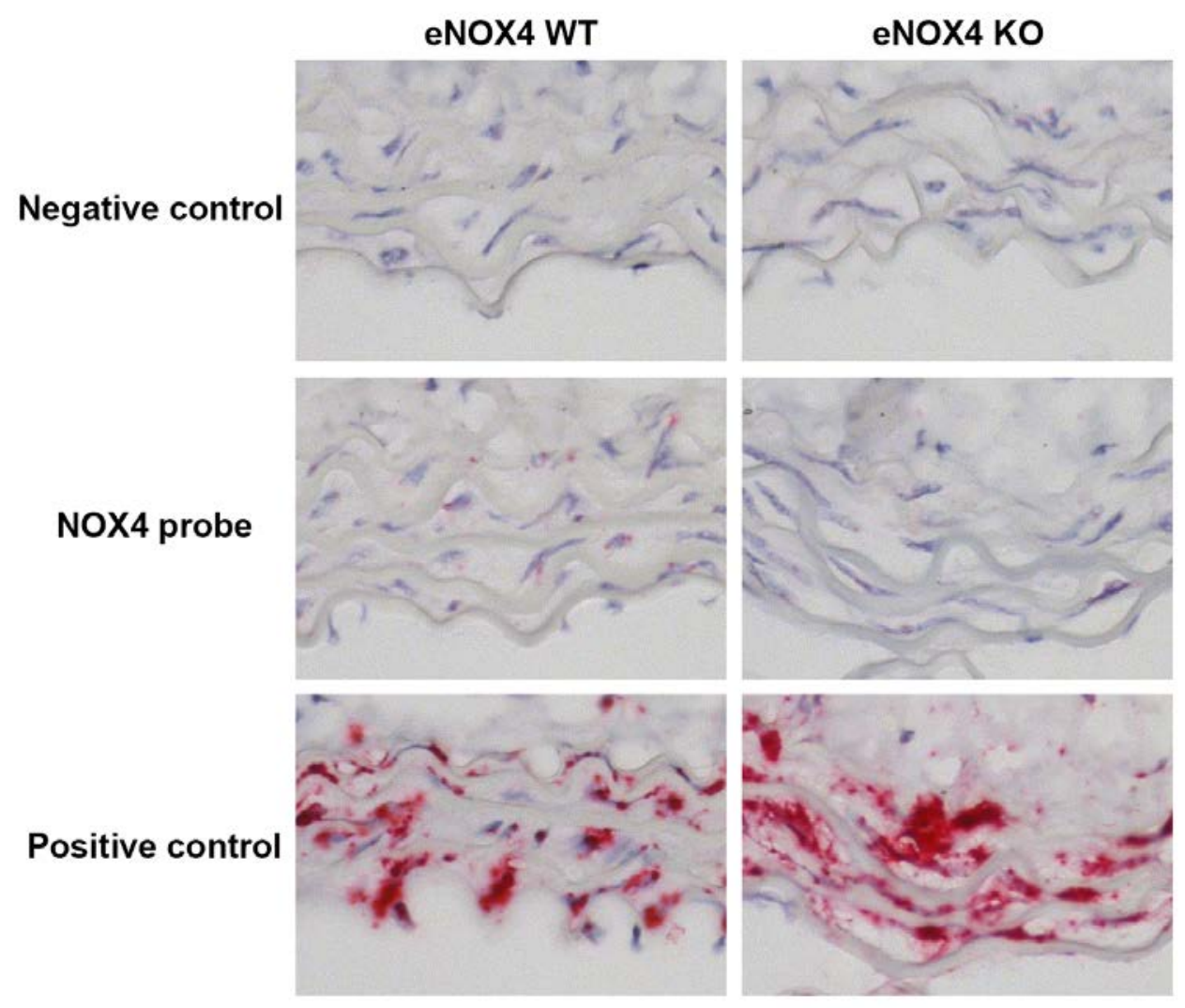

Figure 2. NOX4 mRNA was decreased in the media layer of SNOX4 KO (compared to NOX4 WT mice) as shown by the RNA scope technique (middle panel). The upper and lower panels represent negative and positive controls as provided by the manufacturer.
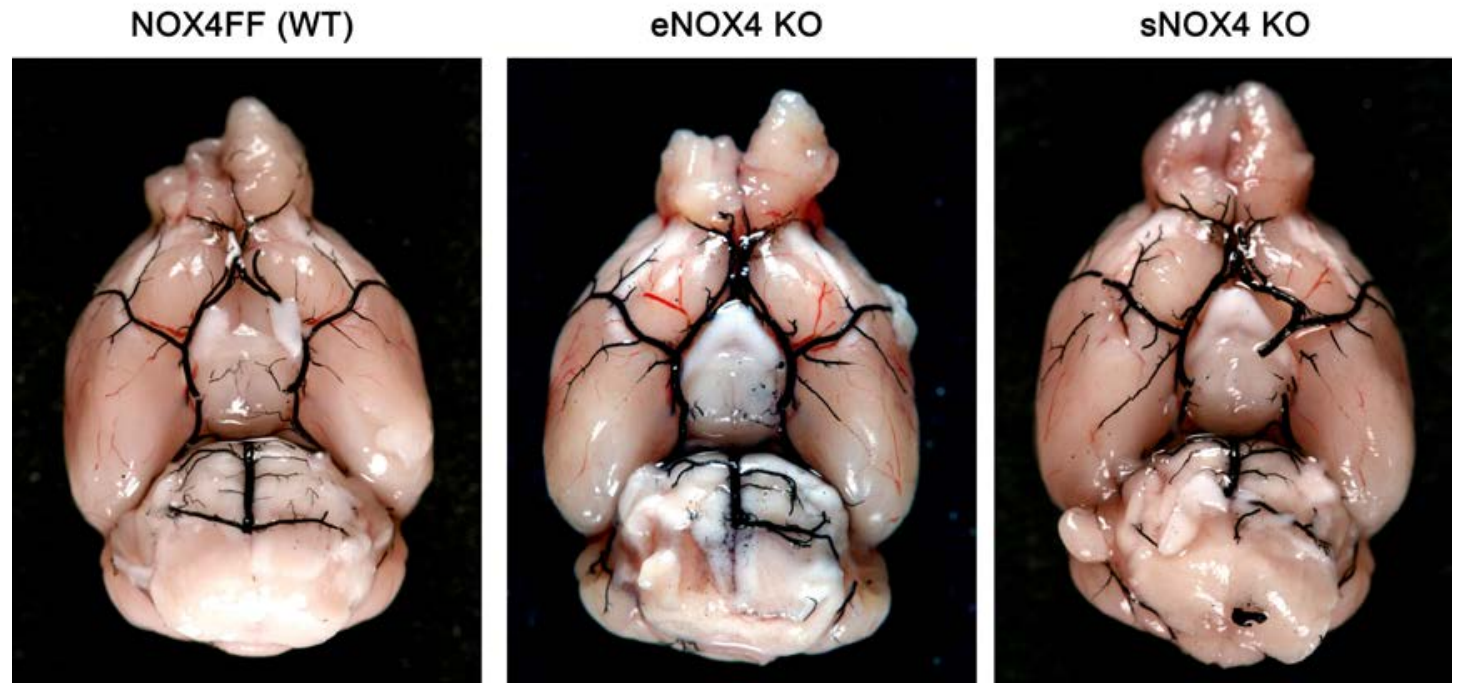

Figure 3. Ink perfused brains show comparable vascular anatomy and a complete Circle of Willis in WT, eNOX4 KO and sNOX4 KO mice. 


\section{Physiological parameters}

Systemic blood pressure, as well as heart rate in the endothelial cell specific NOX4 $\mathrm{KO}$ mice (BP 70.2 $\pm 3.0 \mathrm{mmHg}, \mathrm{HR} 527 \pm 17 \mathrm{bpm}, \mathrm{n}=5$ ) were unchanged compared to WT control mice (BP 72.4 $\pm 5.0 \mathrm{mmHg}, \mathrm{HR} 510 \pm 21 \mathrm{bpm}, \mathrm{n}=5$ ). In the smooth muscle cell specific NOX4 KO mice baseline blood pressure and heart rate (BP 73.6 $\pm 1.5 \mathrm{mmHg}, \mathrm{HR} 525 \pm 25 \mathrm{bpm}, \mathrm{n}=8$ ) were also similar to their respective WT mice (BP 69.0 $\pm 2.1 \mathrm{mmHg}$, HR 512 $\pm 17 \mathrm{bpm}, \mathrm{n}=6$ ).

Furthermore, no significant variation in BP/HR occurred during the whole stroke surgery, nor after reperfusion in all four mice groups (Fig. $4 a$ and b). Cerebral blood flow before surgery (baseline), immediately after tMCAO, and 30 minutes after removal of the occluding monofilament (reperfusion) did not differ between the groups at any time point (Fig. 4c). An initial blood flow drop of $50 \%$ was observed after CCA occlusion, which then further decreased after MCA occlusion (70-80\%). Although the observed drop corresponds to what has been reported in the literature, in our case the blood flow was not completely restored 30 minutes after reperfusion. Blood gas analysis did not show any differences in baseline values of $\mathrm{PaO}_{2}, \mathrm{PaCO}_{2}$ and $\mathrm{pH}$ levels in WT $\left(\mathrm{PaO}_{2} 62.6 \pm 3.4 \mathrm{mmHg}, \mathrm{PaCO}_{2} 38.7 \pm 2.9 \mathrm{mmHg}, \mathrm{pH} 7.2 \pm 0.02\right)$ versus the endothelial cell specific NOX4 KO $\left(\mathrm{PaO}_{2} 62.3 \pm 3.9 \mathrm{mmHg}, \mathrm{PaCO}_{2}\right.$ $41.2 \pm 2.6 \mathrm{mmHg}, \mathrm{pH} 7.2 \pm 0.02)$ mice. In smooth muscle cell specific NOX4 KO and WT mice, similar results were found $\left(\mathrm{WT} \mathrm{PaO}_{2} 58.8 \pm 3.6 \mathrm{mmHg}, \mathrm{PaCO}_{2}\right.$ 41.6 $\pm 2.3 \mathrm{mmHg}, \quad \mathrm{pH} 7.2 \pm 0.03, \mathrm{n}=3 ; \quad \mathrm{KO} \quad \mathrm{PaO}_{2} \quad 60.1 \pm 1.6 \mathrm{mmHg}, \quad \mathrm{PaCO}_{2}$ 41.3 $\pm 3.4 \mathrm{mmHg}, \mathrm{pH} 7.2 \pm 0.03$ ) (Fig. 5).

\section{Counterregulation of other NADPH oxidases}

Ischemic stroke induced upregulation of NOX2 mRNA in the ipsilateral side of the brain in eNOX4 mice, but values were not different between WT $(993 \pm 169 \%, n=3)$ and eNOX4 KO (722 $\pm 291 \%, n=5)$ mice (Fig. 5A). In sNOX4 mice, an upregulation of NOX2 mRNA was seen only in the ipsilateral side of the sNOX4 KO $(1220 \pm 399, n=2)$ brain compared to the $\mathrm{KO}$ contralateral site, and was also significantly higher than the ipsilateral side of the sNOX WT mice $(101 \pm 11, n=3)$ (Fig. 5A). The increase in NOX2 mRNA could be explained by an influx of neutrophils into the infarcted brains. NOX1 mRNA was not upregulated in the stroked brains of either eNOX4 WT, eNOX4 KO, sNOX4 WT or sNOX4 KO mice (Fig. 5B). 

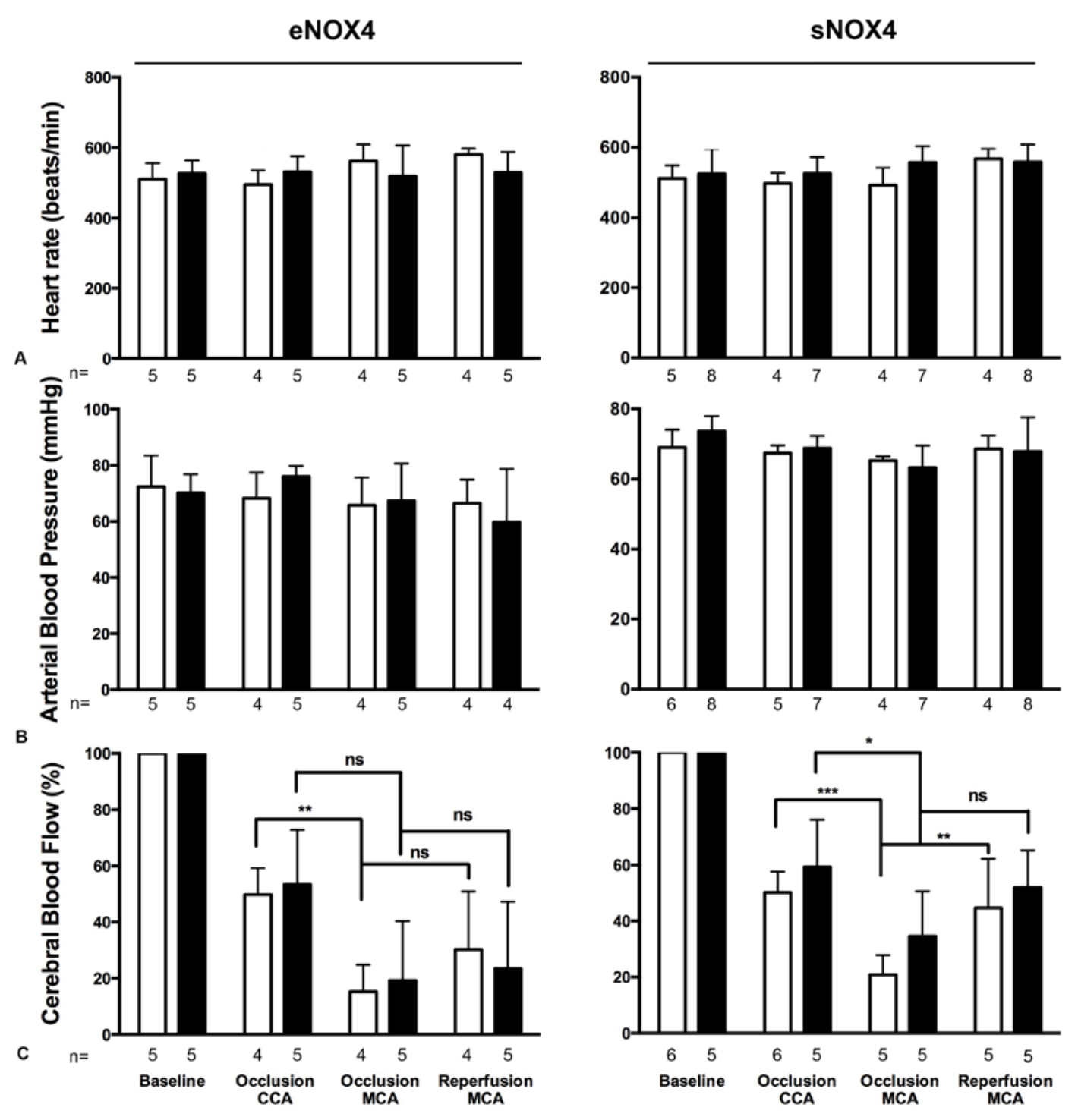

Figure 4. Physiological parameters were not different between the eNOX4 KO and sNOX4 KO (black bars) mice compared to their respective matched WT's (white bars). Parameters were assessed during the stroke surgery at baseline, after occlusion of the common carotid artery (CCA), occlusion of the middle cerebral artery (MCA) and when retracting the filament, allowing reperfusion (reperfusion MCA). A) Heart rate as measured via ECG did not change during any phase of the stroke surgery. B) Blood pressure measured in the aorta was constant during the whole stroke procedure. C) Cerebral blood flow dropped 50\% after the occlusion of the CCA with a further drop to $20-30 \%$ after occlusion of the MCA. After reperfusion the blood flow increased again but not further than 30\% in the eNOX4 and 50\% in the sNOX4 mice. Bars represent mean \pm SEM of $n$ experiments as mentioned below the bars. ${ }^{*} p<0.05^{* \star} p<0.01{ }^{* \star *} p<0.001$ n.s, not significant. 


\section{No role of smooth muscle cell NOX4 in ischemic stroke}

Mice lacking the NOX4 gene specifically in smooth muscle cells (sNOX4 KO) were compared to WT mice not expressing the SMMHC Cre (sNOX4 WT) and mice expressing the SMMHC-Cre but not treated with tamoxifen (sNOX4 KO-tam, data not shown) Twenty-four hours after tMCAO, sNOX4 KO mice showed a similar infarct size $\left(84 \pm 12 \mathrm{~mm}^{3}, \mathrm{n}=9\right)$ as compared to sNOX4 KO-tam $\left(87 \pm 11 \mathrm{~mm}^{3}, \mathrm{n}=10\right)$ and sNOX4 WT (73 $\pm 14 \mathrm{~mm}^{3}, \mathrm{n}=13$ ) (Fig. 7a and b). Also, Bederson and grip test scores were comparable between the three groups (Fig. 7c and d).
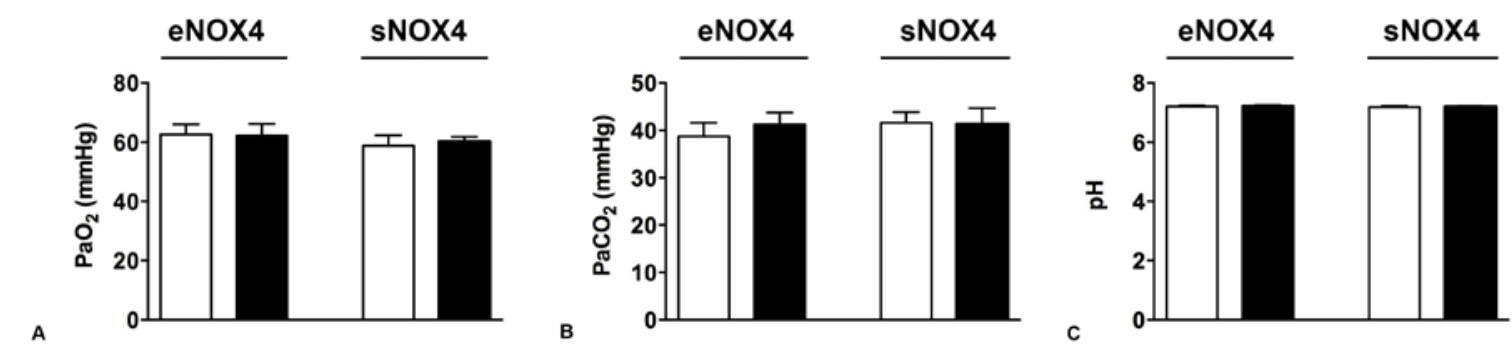

Figure 5. Blood gas analysis did not show differences in oxygen tension (A) carbon dioxide tension (B) or pH level (C) in eNOX4 KO and sNOX4 KO (black bars) versus their matched WT mice (white bars). Bars represent mean \pm SEM of $n=3$ experiments for each group.

Evans blue staining showed more extravasation in the ipsilateral versus the contralateral hemisphere after stroke, with no difference between the ipsilateral side when comparing sNOX4 KO $(1,2 \pm 0,2 \mu \mathrm{g} ; \mathrm{n}=11)$ with sNOX4 WT $(0,9 \pm 0,1 \mu \mathrm{g} ; \mathrm{n}=11)$ mice (Fig. 8).

\section{Endothelial cell NOX4 is a major contributor to inflammation, blood brain barrier disruption and oxidative stress in ischemic stroke.}

Deleting the NOX4 gene in endothelial cells (using a Tie2 specific promotor in a Cre mouse) resulted in a 39\% decrease in infarct size 24 hours after tMCAO (eNOX4 KO 65.9 $\pm 5,0 \mathrm{~mm}^{3}, \mathrm{n}=38$; eNOX4 WT 108.2 $\pm 6.1 \mathrm{~mm}^{3}, \mathrm{n}=28 ; \mathrm{p}<0.0001$ ) (Fig. 7a and b). 

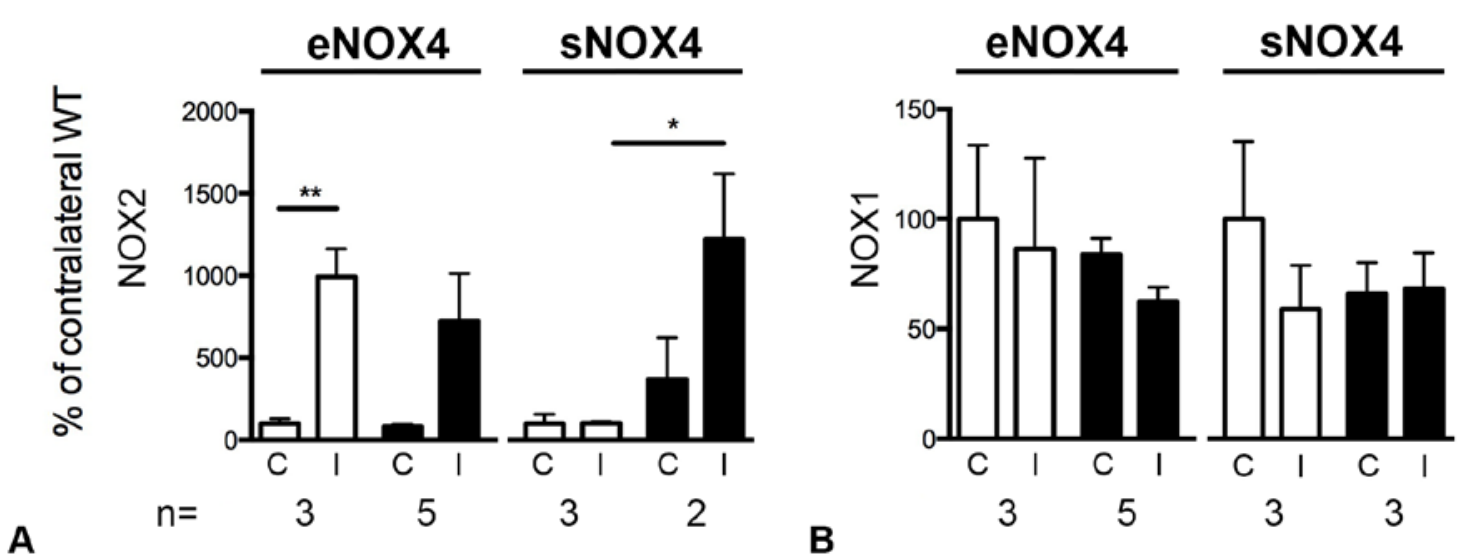

Figure 6. mRNA levels of NADPH oxidase 2 (A) was upregulated in the ipsilateral side of eNOX4 KO (black bars) and WT (white bars) mice after stroke. In the sNOX mice, only the sNOX4 KO mice showed an upregulation of NOX2 mRNA. mRNA levels of NADPH oxidase 1 (B) were not changed in either the eNOX4 KO and WT or sNOX4 KO and WT mice. Bars represent mean $\pm S E M$ of $n$-experiments as mentioned below the bars. WT contralateral brain sides were set to $100 \%$ for both the eNOX and sNOX mice. ${ }^{*} p<0.05 * * 0<0.01$

One group of young males was sacrificed 3 days after tMCAO, here the eNOX4 KO mice also showed a decrease in infarct size $\left(80 \pm 4,2 \mathrm{~mm}^{3}, \mathrm{n}=10\right)$ versus eNOX4 WT mice $\left(103 \pm 3,9 \mathrm{~mm}^{3}, \mathrm{n}=8, \mathrm{p}=0.002\right)$. In the pMCAO model, eNOX4 KO mice showed a $28 \%$ decrease in infarct size (eNOX4 KO $80,9 \pm 12,4 \mathrm{~mm}^{3}, \mathrm{n}=7$; eNOX4 WT $\left.111,7 \pm 6,3 \mathrm{~mm}^{3}, \mathrm{n}=8 ; \mathrm{p}=0.04\right)$.

Neurological scoring showed a better Bederson and grip test score in the eNOX4 KO compared to the WT mice, both after $24 \mathrm{~h}$ and 3 days after tMCAO but not after pMCAO (Fig. 7c and d).

Evans blue extravasation showed a disruption of the blood brain barrier in the ipsilateral brain hemisphere of eNOX4 WT mice (ipsilateral $7,7 \pm 1,2 \mathrm{ng} / \mathrm{mg}$ versus contralateral 1,8 $\pm 0,1 \mathrm{ng} / \mathrm{mg})$. In eNOX4 $\mathrm{KO}$ mice the ipsilateral $(3,4 \pm 0,8 \mathrm{ng} / \mathrm{mg})$ and contralateral $(1,5 \pm 0,1 \mathrm{ng} / \mathrm{mg})$ hemisphere showed the same amount of extravasation. The amount of Evans blue in the ipsilateral hemisphere was reduced in the eNOX4 KO versus the eNOX4 WT showing a better preserved integrity of the blood brain barrier ( $p=0.02$, Fig. 4). 


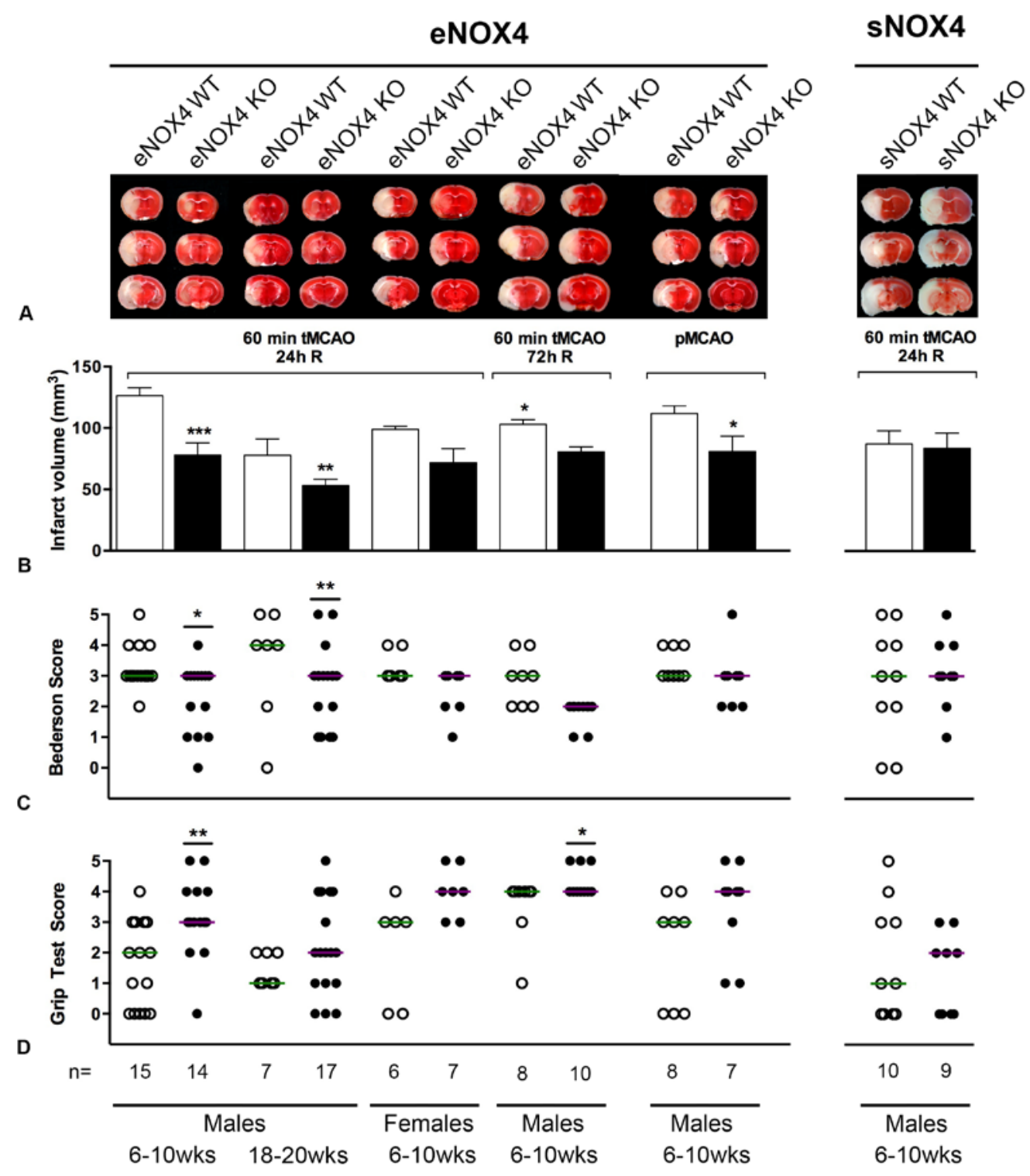

Figure 7. Infarct size and neurological functioning show protection from brain ischemia in eNOX4 KO but not in sNOX4 KO mice. A) Representative TTC staining of three consequent coronal brain sections on day 1 or 3 after tMCAO or day 1 after PMCAO. The TTC staining colours viable tissue red while infarcted tissue stays white. B) Calculated infarct volumes from NOX4 KO (black bars) and control WT (white bars) mice. Bars represent mean \pm SEM of $n$-experiments as mentioned below the bars $\left({ }^{*} P<0.05\right)$. C) Scatter plot and median of Bederson scores showing neurological scorings ranging from 0 (normal) to 5 (severe deficit) on day 1 after tMCAO (open dots, green lines: WT mice; closed dots, purple lines: KO mice). D) Scatter plot and median of grip test scores, ranging from 0 (severe deficit) to 5 (normal). The motor performance was expressed in terms of the ability to reach a post at one or the other side of the wire (open dots, green lines: WT mice; closed dots, purple lines: KO mice). 
A
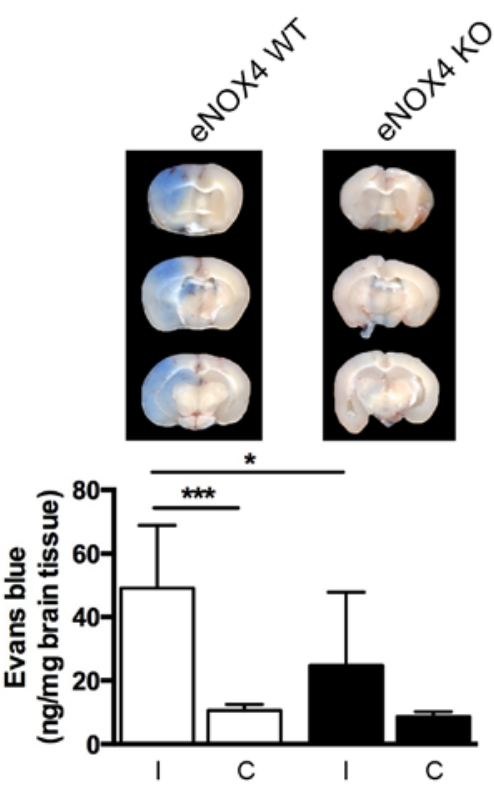
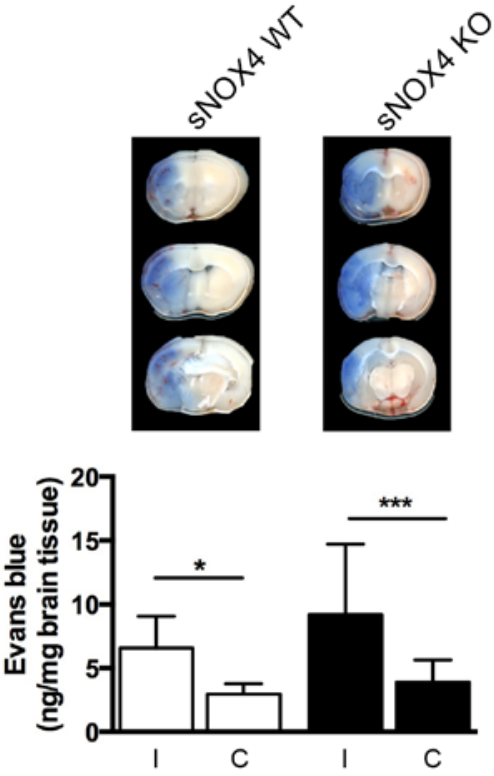

Figure 8. Blood brain barrier integrity 24h after tMCAO was preserved in the eNOX4 KO mice but not in the sNOX4 KO mice (black bars) compared to their respective WT's (white bars). A) Representative pictures showing the extravasation of Evans blue dye into the ipsilateral and contralateral hemispheres of the brain. B) Quantified amount of Evans blue dye (ng Evans blue/mg brain protein tissue) in eNOX4 KO (n=6), eNOX4 WT (n=8), sNOX4 KO $(n=11)$ and sNOX4 WT ( $n=12)$ mice. Bars represent mean $\pm S E M\left({ }^{\star} p<0.05,{ }^{* \star *} p<0.001\right)$.

Oxidative stress was measured using DHE staining on cryosections of infarcted brains. Relative fluorescence intensity was decreased in the ipsilateral brain of eNOX4 KO (10.15 $\pm 0.51, n=5)$ compared to eNOX4 WT $(15.12 \pm 0.07, n=4)$ mice $(p<0.01)($ Fig. 9A)

Finally, neuronal apoptosis was found to be decreased in the stroked brain of eNOX4 KO (140.8 \pm 19.8 cells, $n=4)$ mice compared to their matched WT $(167.4 \pm 3.9$ cells, $\mathrm{n}=5$ ) mice but this did not reach statistical significance (Fig. 9B). 


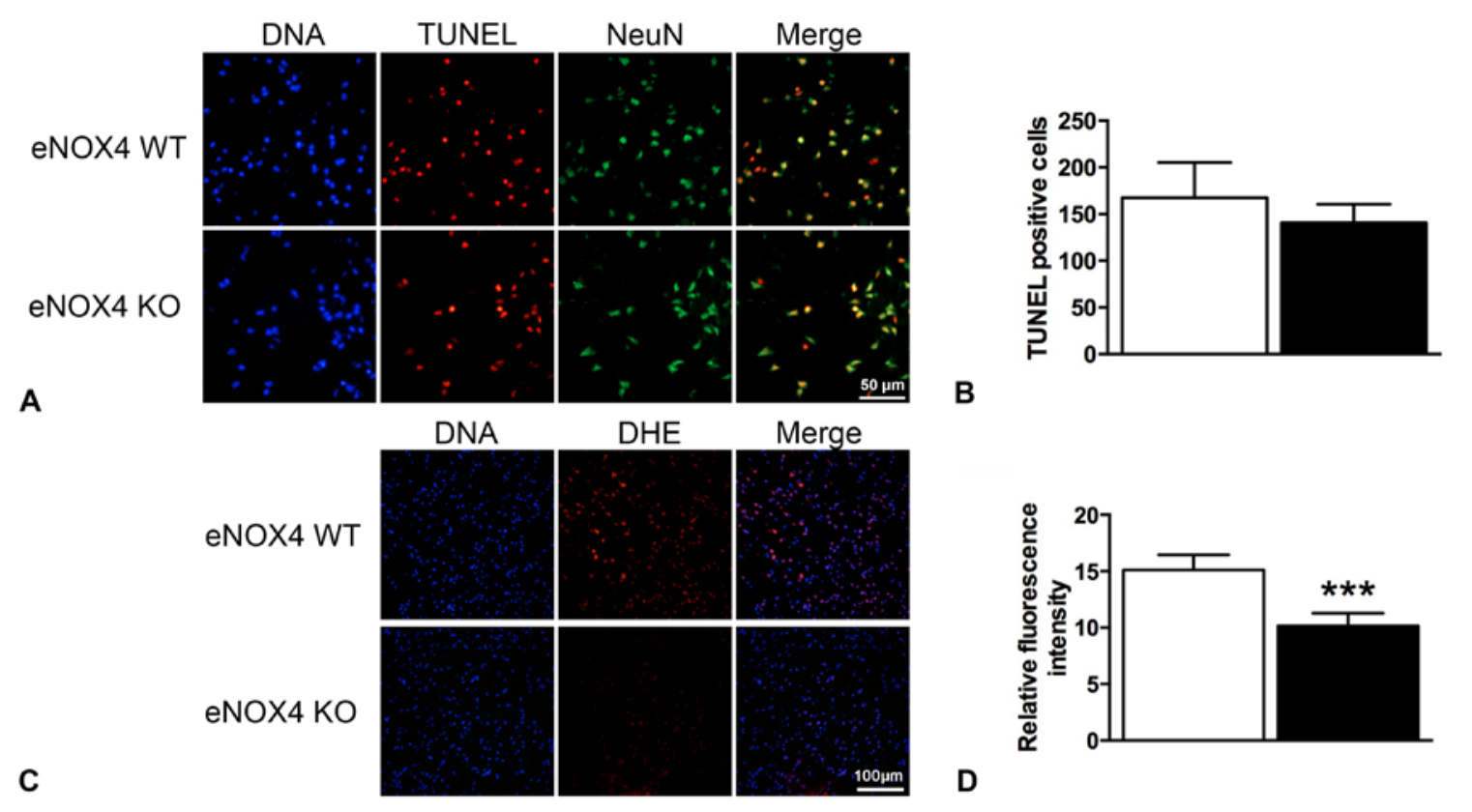

Figure 9. eNOX4 KO (black bars, $n=5$ ) mice show decreased oxidative stress but the same amount of neuronal apoptosis compared to eNOX4 WT (white bars, $n=4$ ) mice. A) Representative pictures of TUNEL and NeuN costaining of the stroked brain. B) Number of TUNEL positive neurons per brain slice. C) Representative pictures of DHE and nuclear staining of the stroked brain. D) Relative fluorescence intensity in stroked brain sections. Bars represent means \pm SEM. (*** $p<0.001$ versus WT)

\section{Possible role for neuronal NOX4 in ischemic damage in the brain}

With no neuron specific NOX4 KO mice yet available, the possible role of neuronal NOX4 was investigated using an in vitro assay. Hippocampal slices of isolated rat brains were subjected to 15 minutes of oxygen-glucose deprivation, followed by 2 hours of reperfusion +/- the NOX inhibitors GKT136901 and VAS2870 in different concentrations.

Cell viability after 15 minutes of OGD and 2 hours of reperfusion was reduced to $67 \pm 2.0 \%$ compared to control. Increasing concentrations of GKT136901 could prevent part of the cell death when added during the 2 hours reperfusion period, which was significant in all but the $0.3 \mu \mathrm{M}$ concentration $(0.1 \mu \mathrm{M} 74 \pm 2.2 \%, 0.3 \mu \mathrm{M}$ $72 \pm 2.2 \%, 1 \mu \mathrm{M} 79 \pm 3.5 \%$ and $3 \mu \mathrm{M} 80 \pm 2.3 \%)$. 
VAS2870 also increased cell viability, but only in the higher concentrations (OGD $65 \pm 4.2 \%, 3 \mu \mathrm{M} 70 \pm 2.4 \%, 10 \mu \mathrm{M} 78 \pm 4.8 \%$ and $30 \mu \mathrm{M} 82 \pm 3.0 \%)$. GKT and VAS are mainly inhibitors of NOX4 thus these data point towards an additional role of neuronal NOX4 in ischemic brain damage. However, this should be confirmed by in vivo experiments in neuronal cell specific NOX4 KO mice.

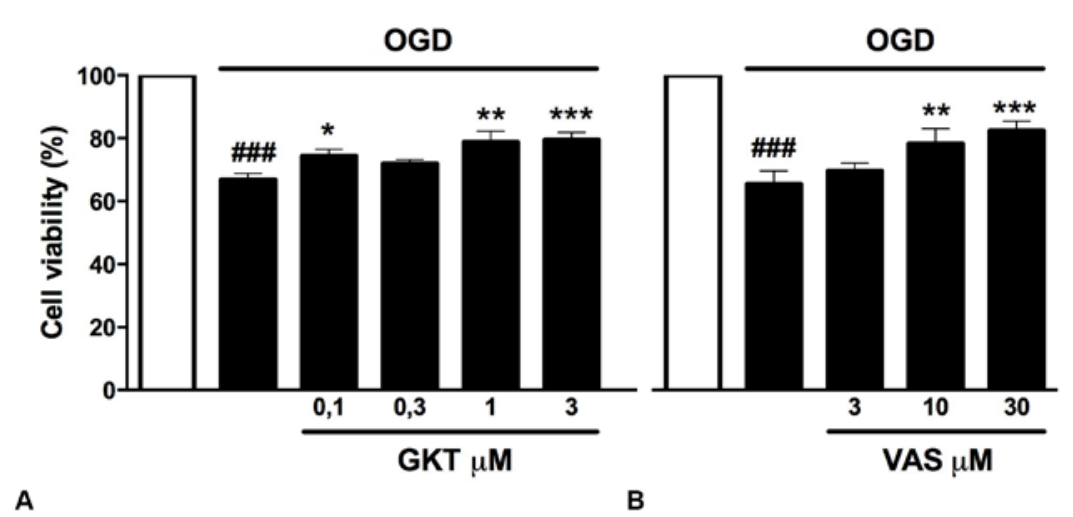

Figure 10. Cell viability of hippocampal brain slices subjected to 15 min oxygen glucose deprivation and 2 hours of reperfusion could be enhanced by the NOX inhibitors GKT136901 or VAS2870. Cell viability was tested using the MTT colorimetric assay with the control values set to $100 \%$. A) Hippocampal slices undergoing OGD (black bars, $n=18$ ) show decreased viability as compared to control (white bars, $n=18$ ). GKT136901 in concentrations of $0,1(n=15), 1.0(n=12)$ and $3(n=6) \mu M$ increased cell viability after OGD. $0.3 \mu M(n=7)$ GKT did not change viability. B) Hippocampal slices undergoing OGD (black bars, $n=7$ ) show decreased viability as compared to control (white bars, $n=7$ ). VAS2870 in a concentration of $3 \mu M(n=7)$ did not affect cell viability after OGD. $10(n=7)$ and $30(N=7) \mu M$ VAS2870 could increase cell viability compared to OGD without inhibitor. Bars represent mean $\pm S E M$. $\# \# p<0.001$ vs non-OGD control, * $p<0.05$ vs OGD without inhibitor, ${ }^{* *} p<0.01$ vs OGD without inhibitor, ${ }^{\star * \star} p<0.001$ vs OGD without inhibitor.

\section{Discussion}

NOX4 is a major source of detrimental ROS in stroke causing both blood brain barrier breakdown and neuronal damage ${ }^{1}$. To clarify whether the protective effect in NOX4 KO mice was primarily an effect of BBB stabilisation or neuroprotection, we created endothelial and smooth muscle cell specific NOX4 KO mice. 
Mice lacking the NOX4 enzyme specifically in endothelial cells showed smaller infarcts and better neurological scoring than their matched WT's indicating a major role for endothelial cell NOX4 in ischemic brain injury. This protection in the eNOX4 $\mathrm{KO}$ mice was not as much as in the global $\mathrm{KO}$, pointing towards an additional role of other cell types. Smooth muscle cell specific NOX4 KO mice did not show a reduction in infarct size excluding SMC as important player in ischemia of the brain. Mechanistically, prevention of the blood brain barrier disruption was found in the eNOX4 KO mice, but not in the sNOX4 KO mice. Blood brain barrier disruption is one of the major consequences of brain ischemia, leading to edema, swelling and additional brain damage. Brain swelling is a very critical complication with ischemic stroke that often needs surgical decompression and has high rates of morbidity and mortality ${ }^{9,10}$. Inhibiting NOX4 clinically after stroke would thus not only limit the acute effects of stroke, but also the brain damage occurring due to edema formation. Neurons also express NOX4, making them a third possible cell type for targeting in stroke. In vitro oxygen glucose deprivation induced cell death in hippocampal slices of rat brain, could be partially prevented by giving the NOX inhibitors GKT136901 and VAS2870. However, both inhibitors have been shown not to be specific for NOX4, with VAS2870 causing thio-alkylation of the RhyR1 receptor ${ }^{11}$ and GKT136901 also inhibiting NOX1 and NOX5 ${ }^{12}$. Thus other NOX isoforms might be involved in the increased viability of hippocampal cells. The OGD in the organotypic hippocampal slices used, mimics the processes going on in ischemia-reperfusion injury more closely than in normal cell culture ${ }^{13-16}$, but still the interplay between different organ systems and for example influx of cells, cannot be addressed in vitro. The pending results with neuron-specific NOX4 KO mice will definitely clarify a possible role of neuronal NOX4. However, presently, all indications argue for a predominant endothelial role of NOX4, which is ideal for a potential treatment, as the BBB will not impose a problem for drug accessibility. 


\section{Methods}

\section{Cell specific NOX4 KO mice generation}

Constitutive NOX4 KO mice and floxed NOX4 mice were generated as described ${ }^{1}$. To generate endothelial-specific NOX4 KO (eNOX4 KO) mice, female mice (homozygous for the floxed NOX4 gene) were bred with male mice (C57BI6 strain background) that express the Cre recombinase gene under control of the endothelialcell specific Tie 2 promotor ${ }^{17}$. As the Cre is located on the $X$ chromosome, all males used for this breeding were hemizygous for the Cre gene $\left(\mathrm{Cre}^{+/ y}\right)$. During breeding, only males that bear the Cre gene were selected for future breeding rounds, while females were not allowed to bear the Cre. For experiments Cre positive (eNOX4 KO) young and adult males and females as well as Cre negative littermates as control animals (=floxed NOX4 mice, eNOX4 WT) were used.

Smooth muscle cell-specific NOX4 KO mice were generated in an analogous way using SMMHC-CreER ${ }^{T 2}$ mice. These mice are described in ${ }^{18}$. Deletion of NOX4 in SMC was induced using Tamoxifen as described ${ }^{19}$. For tMCAO, male, 8-10 weeks old Cre positive mice treated with tamoxifen (sNOX4 KO) were used. Both Cre positive mice not treated with tamoxifen (sNOX4 KO-tam) and NOX4FF Cre negative mice (sNOX4 WT) treated with tamoxifen were used as control groups.

\section{Vessel anatomy}

Anatomy of the cerebral vasculature was assessed by deeply anesthetising mice with $\mathrm{CO}_{2}$ and transcardially perfusing them with $4 \%$ paraformaldehyde (4\% PFA). Three milliliter black ink diluted in 4\% PFA (1:5 v/v) was injected; brains were carefully removed and fixed in 4\% PFA overnight. The Circle of Willis and major arteries were then examined under a microscope.

\section{Stroke surgery (MCAO model)}

The model has previously been established by using cerebral blood flow monitoring above the territory of the middle cerebral artery (MCA) in order to visualize a drop of $70-80 \%$ in blood flow after successful vessel occlusion by Laser Doppler (Moor Instruments Ltd., Devon, UK). 
After administration of a painkiller (buprenorphine s.c. $0,05 \mathrm{mg} / \mathrm{kg}$, repeated every 12 hours), animals were anesthetized with isoflurane (induction 5\% in air, maintenance $1.5-2.5 \%$ in air). Anaesthesia was maintained by spontaneous ventilation of isoflurane. The animal was placed on a heating-pad (UNO Roestvaststaal BV, Zevenaar, NL) and rectal temperature was maintained at $37.0^{\circ} \mathrm{C}$ using a feedbackcontrolled infrared lamp. Focal cerebral ischemia was induced using an intraluminal filament technique. Using a surgical microscope (Wild M5A, Wild Heerbrugg, Gais, $\mathrm{CH}$ ), a midline neck incision was made and the right common and external carotid arteries were isolated and permanently ligated. A microvascular clip was temporarily placed on the internal carotid artery. A silicon-coated nylon monofilament (size 6-0, Doccol Corporation, Redlands, CA, USA) was inserted through a small incision into the common carotid artery and advanced into the internal carotid artery until a resistance is felt. The tip of the monofilament should be located intracranially at the origin of the right middle cerebral artery and thereby interrupting blood flow. The filament was held in place by a tourniquet suture that has been prepared before to prevent dislocation during the ischemic period and the wound was closed. Reperfusion was initiated 1 hour after occlusion by monofilament removal. After the surgery, wounds were carefully sutured and animals were allowed to recover from surgery in a temperature-controlled cupboard. No animal dropouts occurred during surgery; the observed mortality was rather intrinsic to the stroke pathology (as verified by autopsy).

\section{Physiological parameters during stroke model}

A separate group of mice was used to measure physiological parameters during the stroke tMCAO model at baseline, after ligation of the common carotid artery, at blockage of the MCA and after reperfusion.

Laser-Doppler flowmetry (Moor Instruments, UK) was used to monitor regional cerebral blood flow in isoflurane-anesthetized mice before. For this procedure a small incision was made in skin overlaying the skull and a flexible laser-doppler probe (model P10d, Moor Instruments, UK) was positioned and glued with cyanoacrylate (loctite 4161,Technologies House) and glue accelerator (Instaset,bSi Bob Smith incorporation) perpendicular on the superior portion of the temporal bone. 
This position corresponds to the core of the ischemic area. Blood flow is expressed as percentage relative to baseline values.

Continuous blood pressure and heart rate monitoring were performed by inserting a stretched PE10 tubing (Rubber bv) into the abdominal aorta via the femoral artery. Blood pressure and heart rate were measured by using the MPAQ system (IDEE, Maastricht University, The Netherlands).

At baseline, blood gas analysis was performed by drawing $100 \mu \mathrm{L}$ blood from the left cardiac ventricle of anesthetised mice using a heparinised syringe. $\mathrm{PaO}_{2}, \mathrm{PaCO}_{2}$ and $\mathrm{pH}$ were determined using an $\mathrm{ABL} 77$ automated blood gas analyser (Radiometer).

\section{Neurological behaviour}

The mice were assessed for neurological behaviour just before sacrifice to determine the final functional status of the animal. Neurological deficits of the mice that had undergone stroke surgery were measured in a blinded manner on a 0 to 5 scale by using the Bederson Score ${ }^{20}$ with the following definitions:

Score 0 , no apparent neurological deficits; 1 , body torsion and forelimb flexion; 2, right side weakness and thus decreased resistance to lateral push; 3 , unidirectional circling behaviour; 4 , longitudinal spinning; 5 , no movement.

\section{Motor function}

Prior to sacrifice, the mice were also scored for neurological motor deficit according to the Grip Test ${ }^{21}$. Each mouse was given a discrete value from 0 to 5 . This score is used to evaluate motor function and coordination. The apparatus is a metal rod (0.22 cm diameter, $50 \mathrm{~cm}$ length) between two vertical supports at a height of $40 \mathrm{~cm}$ over a flat surface. The animal is placed mid-way on this rod and is rated according to the following system:

Score 0 , falls off; 1 , hangs on to string by one or both fore paws; 2 , as for 1 , and attempts to climb on to string; 3 , hangs on to string by one or both fore paws plus one or both hind paws; 4 , hangs on to string by fore and hind paws plus tail wrapped around string; 5 , escape (towards the supports). 


\section{Blood brain barrier function}

To determine the permeability of the cerebral vasculature and brain edema, $2 \%$ Evans blue tracer (Sigma Aldrich, Germany) diluted in $0.9 \% \mathrm{NaCl}$ was injected intravenously $2 \mathrm{~h}$ after the induction of tMCAO. We then performed planimetric measurements (ImageJ software, National Institutes of Health, USA) on 2 mm-thick sections of the brain parenchyma stained by Evans blue to calculate edema volumes.

\section{Tissue sampling}

The mice were sacrificed by decapitation. The brain was carefully dissected out, freed from the dura mater and placed in a coronal mouse brain slicer (Zivic Instruments, Pittsburgh, PA, USA). Three sequential, $2 \mathrm{~mm}$-thick coronary slices of the brain were cut with razor blades directed by the brain matrix.

\section{Infarct Volume Measurements}

The ischemic lesion was measured 24 hours after MCAO. The slices were soaked for 10 min in a freshly-prepared solution of 2\% 2,3,5- triphenyltetrazolium hydrochloride (TTC, Sigma-Aldrich, Zwijndrecht, NL) in PBS ( $\mathrm{pH} 7.4$ ) in a small Petri dish, maintained at $37^{\circ} \mathrm{C}$ in a heater. Excess TTC was then drained, and the slices were washed with PBS and then photographed under the microscope (Dino-Lite Microscope Eyepiece Camera AM 423X). Each brain section was photographed with a ruler. The digital photographs of the TTC stained brain sections were imported into an image analysis program using the software "Leica QWin Pro" for infarct volume measurement. The program quantitatively measures (in $\mathrm{mm}^{2}$ ) the areas of the infarcted region, the areas of the left and right hemisphere and the total area of the brain slice. Total infarct volume was calculated by adding up infarct volumes of the different sections:

$V_{\text {infarct }}$ section $1\left(\mathrm{~mm}^{3}\right)=$ infarct area section $1\left(\mathrm{~mm}^{2}\right) \times$ slice thickness $(2 \mathrm{~mm})$ 
Infarct volumes are corrected for brain oedema according to the following equation:

$$
\mathrm{V}_{\text {corrected }}\left(\mathrm{mm}^{3}\right)=\mathrm{V}_{\text {infarct }} \mathrm{x}\left(1-\left(\mathrm{V}_{\mathrm{i}}-\mathrm{V}_{\mathrm{c}}\right) / \mathrm{V}_{\mathrm{c}}\right)
$$

With $\mathrm{V}_{\mathrm{i}}-\mathrm{V}_{\mathrm{c}}$ representing the volume difference between the ipsilateral and contralateral hemisphere and $\left(\mathrm{V}_{\mathrm{i}}-\mathrm{V}_{\mathrm{c}}\right) / \mathrm{V}_{\mathrm{c}}$ expressing this difference as \% of the control hemisphere. Brain edema volume can be calculated by subtracting corrected from uncorrected infarct volumes ${ }^{22}$.

\section{Oxidative stress and apoptosis}

The presence of ROS was determined using dihydroethidium (Sigma, stock solution $2 \mathrm{mM})$ staining in coronal brain sections taken from identical regions $(-0.5 \mathrm{~mm}$ from bregma) of the different animal groups ${ }^{1}$. Briefly, frozen sections were incubated in $2 \mu \mathrm{M}$ DHE for 30 minutes at $37^{\circ} \mathrm{C}$, washed three times with PBS and incubated with Hoechst (Hoechst 33342, Sigma-Aldrich) 2ng/ml for $10 \mathrm{~min}$. Grayscale analysis was performed on inverted digital images by using Scion Image (Version 4.0.3.2, for Win95/98 and Windows NT, Scion Corporation), and the number of reactive oxygen species positive cells per $\mathrm{mm}^{2}$ was quantified.

Apoptotic neurons were visualized by TUNEL as described in ${ }^{1}$. Paraffin-wax embedded slices were de-waxed for $30 \mathrm{~min}$ in xylol and rehydrated in ethanol. The TUNEL in situ death detection kit TMR red (Roche, Switzerland) was used according to the manufacturer's instructions. Co-staining was done with the neuronal marker NeuN (mouse neuN MAB377, clone A60; Millipore, Billerica, USA, 1:1,000) using a secondary goat-anti-mouse antibody (Invitrogen, Germany; Alexa fluor 488, 1:3000, A11001). Afterwards, slices were washed and subsequently covered with AquaTec (Merck, Darmstadt, Germany). Pictures were collected by immunofluorescence microscopy (Axiophot; Zeiss, Jena, Germany). Quantification of TUNEL-positive cells per $\mathrm{mm}^{2}$ was performed by using ImageJ software (http://rsb.info.nih.gov/ij/). 


\section{VSMC and EC isolation}

Vascular smooth muscle cells from aortas of sNOX4 KO and WT mice were isolated. 4-5 aortas of each genotype were stripped from all connective tissue and adventitia and cut longitudinally. The inside of the vessels was scraped with the flat edge of a bent forceps to remove epithelial cells and then cut into $1-2 \mathrm{~mm}$ pieces. The aortic pieces were transferred to a tissue culture tube and digested over 4 hours with $3 \mathrm{mg} / \mathrm{ml}$ collagenase (Sigma) and $25 \mu \mathrm{g} / \mathrm{ml}$ elastase (Sigma) in serum free medium. After digestion the cells were washed and transferred into a laminin coated t25 culture flask. Cells were cultured until passage 5-6 in DMEM (gibco 31966-021) 10\% FBS, $1 \% \mathrm{P} / \mathrm{S}$. Cell were then collected by trypsinisation, centrifuged and pelleted by gentle centrifugation $(330 \times \mathrm{g})$.

Brain capillary endothelial cells (MBCEC) from eNOX4 KO and WT mice were isolated as described in ${ }^{23}$. After sacrificing the mice, forebrains were collected, meninges removed and the tissue was minced and digested with a mixture of $0.75 \mathrm{mg} / \mathrm{ml}$ collagenase CLS2 (Worthington) and 10U/mL DNAse (Sigma) in Dulbecco's modified Eagle medium (DMEM; Sigma) for $1 \mathrm{~h}$ at $37^{\circ} \mathrm{C}$. To remove myelin, the pellet was resuspended in BSA-DMEM $(20 \% \mathrm{w} / \mathrm{v})$ and centrifuged (1000x g, $20 \mathrm{~min}$ ). The pellet was resuspended and further digested with $1 \mathrm{mg} / \mathrm{ml}$ collagenase-dispase (Roche) and $10 \mathrm{U} / \mathrm{ml}$ SNAse in DMED for $1 \mathrm{~h}$ at $37^{\circ} \mathrm{C}$. Microvascular endothelial capillaries were separated on a 33\% continuous Percoll gradient, collected and plated in petri-dishes coated with collagen IV/fibronectin (Sigma). Cultures were maintained in DMEM supplemented with $20 \%$ plasma-

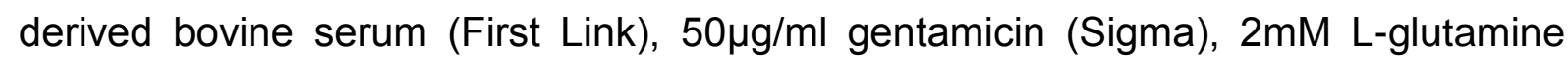
(Sigma), $4 \mu \mathrm{g} / \mathrm{ml}$ puromycin (Alexix $\mathrm{GmbH}$, Grünberg, Germany) and $1 \mathrm{ng} / \mathrm{ml}$ basic fibroblast growth factor (Roche).

\section{RNA scope}

Five $\mu \mathrm{m}$ thick paraffin embedded sections of aortas were stained for NOX4 mRNA (mouse NOX4 probe to exon 14 and 15, custom made by Advanced Cell Diagnostics) using the RNAscope kit (RNAscope 2.0 FFPE reagent kit-RED, cat\# 310036 Advanced Cell Diagnostics) according to the protocol of the manufacturer. 
DapB (cat\# 310043) and Mm UBC (cat\# 310771) were used as negative and positive controls respectively. Counterstaining was performed using haematoxylin.

\section{$R T-P C R$}

RT-PCR was performed using Red Taq Mix (Applied biosystems) on cDNA from isolated endothelial cells and carotid arteries from the eNOX4 KO and WT mice according to the following protocol: $2 \min 94^{\circ} \mathrm{C}, 35 \mathrm{x}$ (30s $94^{\circ} \mathrm{C} / 30 \mathrm{~s} 59^{\circ} \mathrm{C} / 60 \mathrm{~s} 72^{\circ} \mathrm{C}$ ), $10 \mathrm{~min} \quad 72^{\circ} \mathrm{C}, \quad \infty 4^{\circ} \mathrm{C}$. Primers sequences were as follows: GCTGGAGGCATTGGAGTCACTC and GTCCACAGCAGAAAACTCCAACTG (Thermo Scientific).

\section{qPCR}

RNA extraction of the isolated VSMC and MBCEC or stroked brains was done using the RNeasy MiniKit (Qiagen, cat. no 74104) or RNeasy Fibrous Tissue Minikit (Qiagen, cat. no 74704) according to the protocol of the manufacturer. mRNA concentration was determined using the Nanodrop 2000C device (Thermo Scientific) cDNA was then synthesized using the Applied Biosystems High capacity cDNA Reverse Transcriptase kit.

NOX1, 2 and 4 mRNA expression was quantified using real-time PCR and the TaqMan system (TaqMan Gene Expression Arrays for murine NOX1 (assay ID Mm00549170_m1), NOX2 (assay ID Mm01287743_m1) and NOX4 (assay ID Mm00479246_m1), Life Technologies), using 18S rRNA (TaqMan Predeveloped Assay Reagents, assay ID Hs99999901_s1, Life Technologies) to normalize the amount of sample RNA.

\section{Hippocampal brain slices}

In vitro damage caused by oxygen-glucose deprivation/re-oxygenation and the protection elicited by VAS2870 and GKT136901 were studied in acutely isolated rat hippocampal slices. Brains from 2-3 months old adult male Sprague-Dawley rats (250-300 g) were isolated as described previously ${ }^{24-26}$. 
Rats were quickly decapitated under sodium pentobarbital anaesthesia $(60 \mathrm{mg} / \mathrm{kg}$, i.p.), forebrains were rapidly removed from the skull and placed into ice-cold Krebs bicarbonate dissection buffer ( $\mathrm{pH}$ 7.4), containing (in $\mathrm{mM}$ ): $\mathrm{NaCl} 120, \mathrm{KCl} 2, \mathrm{CaCl}_{2}$ $0.5, \mathrm{NaHCO}_{3} 26, \mathrm{MgSO}_{4}$ 10, $\mathrm{KH}_{2} \mathrm{PO}_{4}$ 1.18, glucose 11 and sucrose 200. The chamber solutions were pre-bubbled with either $95 \% \mathrm{O}_{2} / 5 \% \mathrm{CO}_{2}$ or $95 \% \mathrm{~N}_{2} / 5 \% \mathrm{CO}_{2}$ gas mixtures, for at least 30 min before slice immersion, to ensure $\mathrm{O}_{2}$ saturation or removal.

The hippocampi were quickly dissected and $300 \mathrm{~mm}$ thick slices were cut using a Mcllwain Tissue Chopper. Then, the slices were transferred to vials containing a sucrose-free dissection buffer, bubbled with $95 \% \mathrm{O}_{2} / 5 \% \mathrm{CO}_{2}$ in a water bath at room temperature for $45 \mathrm{~min}$, to allow tissue recovery (equilibration period).

Oxygen and glucose deprivation was induced by incubating the slices for a $15 \mathrm{~min}$ period in a glucose-free Krebs solution, equilibrated with a 95\% $\mathrm{N}_{2} / 5 \% \mathrm{CO}_{2}$ gas mixture; glucose was replaced by 2-deoxyglucose. Slices incubated for $15 \mathrm{~min}$ in a modified Krebs solution (in $\mathrm{mM}: \mathrm{NaCl} 120, \mathrm{KCl} 2, \mathrm{CaCl}_{2} 2, \mathrm{NaHCO}_{3} 26, \mathrm{MgSO}_{4} 1.19$,

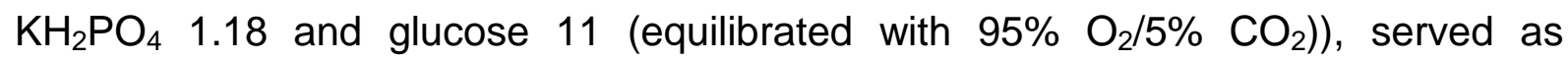
controls.

After the OGD period, the slices were returned back to an oxygenated normal Krebs solution containing glucose for 2 hours (re-oxygenation period), during which VAS2870 or GKT136901 were added as treatments.

\section{Cell viability of the hippocampal slices}

Hippocampal cell viability was determined using the colorimetric MTT assay ${ }^{27}$. Hippocampal slices were collected immediately after the re-oxygenation period and were incubated with MTT $(0.5 \mathrm{mg} / \mathrm{ml})$ in Krebs bicarbonate solution for $30 \mathrm{~min}$ at $37^{\circ} \mathrm{C}$. The tetrazolium ring of MTT can be cleaved by active dehydrogenases in viable cells, producing a precipitated formazan derivative. This formazan derivative was solubilized by adding $200 \mathrm{ml}$ DMSO. The optical density was measured spectrophotometrically at $540 \mathrm{~nm}$ using a micro plate reader. Absorbance values obtained in control slices were set to $100 \%$ viability. 


\section{ACKNOWLEDGMENTS}

We gratefully thank Dr. M. García-López and A. Casas-Guijarro from Universidad Autonóma de Madrid for performing the hippocampal slice experiments.

\section{References}

1. Kleinschnitz, C. et al. Post-stroke inhibition of induced NADPH oxidase type 4 prevents oxidative stress and neurodegeneration. PLOS Biol. 8, (2010).

2. Irani, K. Oxidant signaling in vascular cell growth, death, and survival : a review of the roles of reactive oxygen species in smooth muscle and endothelial cell mitogenic and apoptotic signaling. Circ Res 87, 179-183 (2000).

3. Dröge, W. Free radicals in the physiological control of cell function. Physiol. Rev. 82, 47-95 (2002).

4. Wolin, M. S. Reactive oxygen species and the control of vascular function. Am. J. Physiol. Heart Circ. Physiol. 296, H539-49 (2009).

5. Andreadou, I., Iliodromitis, E. K., Farmakis, D. \& Kremastinos, D. T. To prevent, protect and save the ischemic heart: antioxidants revisited. Expert Opin Ther Targets 13, 945-956 (2009).

6. Vivekananthan, D. P., Penn, M. S., Sapp, S. K., Hsu, A. \& Topol, E. J. Use of antioxidant vitamins for the prevention of cardiovascular disease: metaanalysis of randomised trials. Lancet 361, 2017-2023 (2003).

7. Bjelakovic, G., Nikolova, D., Gluud, L. L., Simonetti, R. G. \& Gluud, C. Mortality in randomized trials of antioxidant supplements for primary and secondary prevention: systematic review and meta-analysis. Jama 297, 842-857 (2007).

8. Wingler, K. et al. NOX 1, 2, 4, 5: Counting out oxidative stress. Br J Pharmacol Epub ahead of print, (2011).

9. Wijdicks, E. F. M. et al. Recommendations for the management of cerebral and cerebellar infarction with swelling: a statement for healthcare professionals from the American Heart Association/American Stroke Association. Stroke 45, 1222-1238 (2014).

10. Walcott, B. P. et al. Outcomes in severe middle cerebral artery ischemic stroke. Neurocrit Care 21, 20-26 (2014). 
11. Sun, Q. A., Hess, D. T., Wang, B., Miyagi, M. \& Stamler, J. S. Off-target thiol alkylation by the NADPH oxidase inhibitor 3-benzyl-7-(2-benzoxazolyl)thio1,2,3-triazolo[4,5-d]pyrimidine (VAS2870). Free Radic Biol Med 52, 1897-1902 (2012).

12. Altenhöfer, S., Radermacher, K. A., Kleikers, P., Wingler, K. \& Schmidt, H. H. Evolution of NADPH oxidase inhibitors: Selectivity and mechanisms for target engagement. Antioxid Redox Signal (2014). doi:10.1089/ars.2013.5814

13. Newell, D. W., Barth, A., Papermaster, V. \& Malouf, A. T. Glutamate and nonglutamate receptor mediated toxicity caused by oxygen and glucose deprivation in organotypic hippocampal cultures. J. Neurosci. 15, 7702-7711 (1995).

14. Newell, D. W., Malouf, A. T. \& Franck, J. E. Glutamate-mediated selective vulnerability to ischemia is present in organotypic cultures of hippocampus. Neurosci. Lett. 116, 325-330 (1990).

15. Gähwiler, B. H. Organotypic cultures of neural tissue. Trends Neurosci. 11, 484-489 (1988).

16. Gähwiler, B. H., Capogna, M., Debanne, D., McKinney, R. A. \& Thompson, S. M. Organotypic slice cultures: a technique has come of age. Trends Neurosci. 20, 471-477 (1997).

17. Kisanuki, Y. Y. et al. Tie2-Cre transgenic mice: a new model for endothelial cell-lineage analysis in vivo. Dev. Biol. 230, 230-242 (2001).

18. Wirth, A. et al. G12-G13-LARG-mediated signaling in vascular smooth muscle is required for salt-induced hypertension. Nat. Med. 14, 64-68 (2008).

19. Groneberg, D. et al. Smooth muscle-specific deletion of nitric oxide-sensitive guanylyl cyclase is sufficient to induce hypertension in mice. Circulation 121, 401-409 (2010).

20. Bederson, J. B. et al. Rat middle cerebral artery occlusion: evaluation of the model and development of a neurologic examination. Stroke 17, 472-476 (1986).

21. Moran, P. M., Higgins, L. S., Cordell, B. \& Moser, P. C. Age-related learning deficits in transgenic mice expressing the 751-amino acid isoform of human beta-amyloid precursor protein. Proc Natl Acad Sci U S A 92, 5341-5345 (1995). 
22. Kraft, P. et al. Deficiency of vasodilator-stimulated phosphoprotein (VASP) increases blood-brain-barrier damage and edema formation after ischemic stroke in mice. PLoS ONE 5, e15106 (2010).

23. Weidenfeller, C., Schrot, S., Zozulya, A. \& Galla, H.-J. Murine brain capillary endothelial cells exhibit improved barrier properties under the influence of hydrocortisone. Brain Res. 1053, 162-174 (2005).

24. Novel multitarget ligand ITH33/IQM9.21 provides neuroprotection in in vitro and in vivo models related to brain ischemia. 67, 403-411 (2013).

25. Egea, J. et al. Neuroprotection afforded by nicotine against oxygen and glucose deprivation in hippocampal slices is lost in alpha7 nicotinic receptor knockout mice. Neuroscience 145, 866-872 (2007).

26. Maroto, M. et al. Multi-target novel neuroprotective compound ITH33/IQM9.21 inhibits calcium entry, calcium signals and exocytosis. Cell Calcium 50, 359369 (2011).

27. Denizot, F. \& Lang, R. Rapid colorimetric assay for cell growth and survival. Modifications to the tetrazolium dye procedure giving improved sensitivity and reliability. J. Immunol. Methods 89, 271-277 (1986). 
Chapter 4

A pre-clinical randomized, controlled trial approach for pharmacological target validation: The case against NOX2 in stroke

Submitted 


\begin{abstract}
Biomedical research suffers from a dramatically poor translational success from basic science into clinical application. For this, ischemic stroke is a prominent example, a disease with high prevalence and only one approved therapeutic drug with over 30 contraindications. Despite this high medical need, over a thousand experimental stroke drugs were unsuccessful. Here, we address the phagocytic oxidative burst NADPH (nicotinamide adenine dinucleotide phosphate) oxidase type 2 (NOX2) for which a profound body of literature exists to be a major target for neuroprotective stroke therapy. However, through a systematic review and metaanalysis, we detected a publication bias suggesting the omission of small, negative studies and a likely overestimation of the role of NOX2. In addition, none of the publications were sufficiently powered to detect at least a minimal relevant infarct size reduction of $40 \%$. To clarify the true role of NOX2 in stroke, we conducted a multi-centre pre-clinical randomized, controlled and fully powered animal trial (pRCT). Under these conditions, we observed a significant reduction of infarct size, but only by $10 \%$. To fully power this difference statistically, 143 animals per study arm (total $\mathrm{n}=286$ ) would be required. More importantly, both the fact that we observed no improved neurofunctional outcomes and its irrelevant contribution to infarct reduction argue against NOX2 as a major pharmacological target in stroke. Further, our results suggest that while meta-analyses are an interim tool to detect missing data, biomedical research for therapeutic target validation would benefit from a fundamental shift towards pRCTs.
\end{abstract}




\section{Significance Statement}

The reproducibility of biomedical research and its translation into clinical application is disappointingly low. To improve drug target validation, we here adopt a method from clinical research and conduct the first preclinical version of multi-centre randomized controlled trial ( $\mathrm{pRCT}$ ). An overwhelming body of literature suggested that the radical-generating NADPH oxidase type 2 (NOX2) is a target for stroke therapy. We identify both a uniform lack of statistical power to detect a clinical relevant reduction in infarct size and a publication bias towards positive findings. Our fully powered PRCT then shows that NOX2 is in fact not a therapeutic target in stroke. Further, our approach suggests improvements in translational research by using more collaborative and higher quality study designs and unbiased publishing. 


\section{Introduction}

Basic biomedical research suffers from major quality and reproducibility ${ }^{1-3}$ issues that then impact on clinical translatability ${ }^{4,5}$. These issues are on top of a general notion that animal models may be less predictive than we thought ${ }^{6,7}$. It has been suggested that this is partly a consequence of suboptimal study design ${ }^{8}$ and analysis ${ }^{9}$, and poor reporting ${ }^{10-12}$. In stroke and its preclinical research, the situation is particularly alarming ${ }^{13}$. Stroke is the third cause of death and number one reason for chronic disability. Despite this high medical need only one drug (recombinant tissue plasminogen activator) is registered to treat ischemic stroke; this even has limited efficacy ${ }^{14}$ and over 30 contraindications, so that $85 \%$ of all stroke patients remain even without any acute drug treatment. This single "successful" stroke drug development stands in sharp contrast to 1,026 failed experimental stroke targets ${ }^{15,16}$, despite several roundtable recommendations to improve the quality of preclinical stroke research ${ }^{17,18}$ including advanced study designs ${ }^{19}$. Consequently, industry scientists have left target discovery and drug development for stroke almost completely non-investigated. Recently, this has led to proposals that preclinical research needs to improve in quality by adopting elements of clinical research, including multi-centre, randomization, blinding and a priori power calculation for relevant outcomes ${ }^{17,20,21}$.

With respect to relevant pre-clinical outcomes, a shift is necessary from surrogates such as infarct size to measurement of neuronal function; with respect to therapeutic approach, patients need a shift from vascular re-canalization to adding on neuroprotection ${ }^{22}$. Oxidative stress or the occurrence of reactive oxygen species (ROS) in increased amounts, in unphysiological places or with unphysiological chemistry, has been suggested to play a major role in neurodegeneration upon ischemic stroke ${ }^{23-25}$. Even under conditions of ischemia ${ }^{26}$, ROS have both protective and deleterious effects ${ }^{27}$, which explains why global anti-oxidant therapy has failed ${ }^{28-30}$. A more promising approach is to target oxidative stress in a manner that leaves essential, physiological ROS formation untouched and inhibits only diseaserelevant enzymatic sources ${ }^{30-32}$. As such a source of ROS, NADPH oxidases (NOX) stand out as they represent the only enzyme family that has ROS formation as its only known function. 
For one hypoxia-inducible isoform, NOX4, this may indeed be the case. However, on another isoform, NOX2, our own previous observation ${ }^{24}$ profoundly disagreed with an overwhelming body of literature. Nine other publications (using a total of 159 animals) suggest that NOX2 is also a major disease target in stroke and its inhibition or knock-out will reduce infarct size by up to $60 \%{ }^{23,33-40}$. As a point of concern, NOX2 is known to play a major role in innate immunity and its deletion causes severe immune deficiencies, in particular with common comorbidities such as diabetes mellitus ${ }^{41}$. As such, the validation of NOX2 for stroke would need to be validated beyond doubt before entering any clinical trial.

Here we learn from the successes of clinical research and implement for the first time a recent suggestion ${ }^{21}$ to conduct pharmacological target validation research as preclinical randomized controlled trials (pRCTs). The outcomes have implications for stroke research and preclinical animal research in general.

\section{Results}

\section{Systematic review and meta-analysis suggest a role of NOX2 in stroke}

Because of some disagreeing results in the literature on the role of NOX2 in stroke, we first conducted a systematic review (for search strategy see SI Appendix Table S1, SI Methods Fig. S1), followed by a meta-analysis of 13 pre-clinical animal studies (18 experiments) on NOX2 in experimental stroke, using mostly transient ischemia models (for study characteristics see SI Appendix Table S2) ${ }^{23,24,33-40,42-44}$. Heterogeneity was relatively high $\left(I^{2}=73 \%\right.$, ) as expected given the large variations in study designs and methodological quality (SI Appendix Table S1, Fig. S1). All studies on NOX2 in stroke were conducted in mice. One study created a permanent occlusion ${ }^{45}$, and all studies but six ${ }^{23,35,37,39,43,44}$ studied neurological outcome $24 \mathrm{~h}$ after ischemia. Indeed, we found that reported size for NOX2 KO vs. NOX2 WT infarct size was significantly smaller (Fig. 1A). NOX2 KO mice also showed a small but significant decreased neurological score compared to WT mice (Fig 1B), which implicates an improved neurological function (SMD -0.67 $[-1,17 ;-0,16] ; n=9$, $\mathrm{p}=0.010$ ). Calculation of mean differences ${ }^{46-48}$ was not possible because of the heterogeneity in study designs (i.e. species) and the variety of scales used to determine the outcomes (see Methods). 
Without present study

With present study

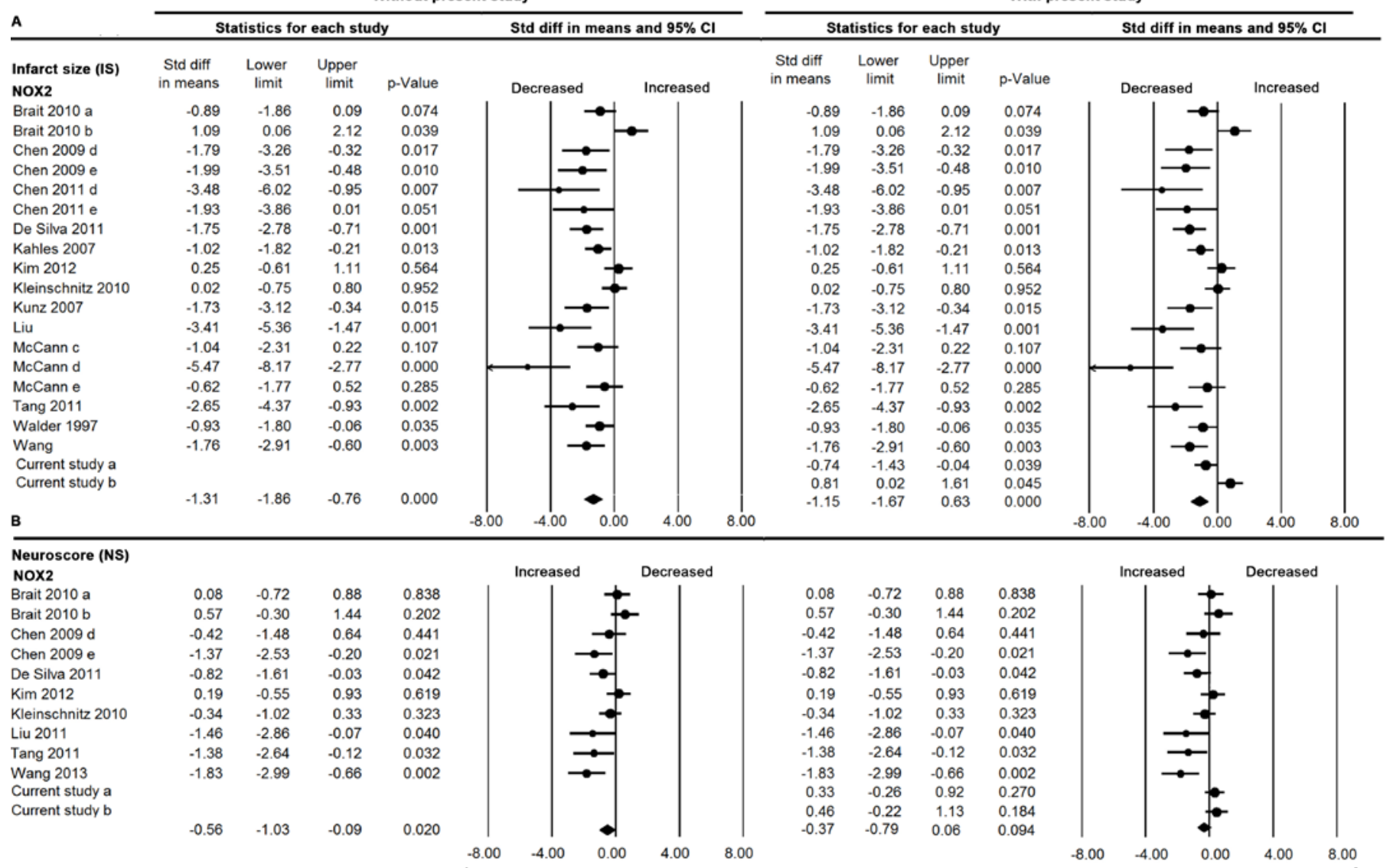

Figure 1. Meta-analysis of the overall effect of NOX2 on infarct size and neurological score in experimental stroke. Studies included are shown on the left and analysed in two forest plots, either without (left section) or with (right section) the data of the here presented randomized, controlled, blinded study. Subgroups within one study are depicted separately with the following coding: a, female gender; $b$, male gender; $c$, short ischemic time; $d$, medium ischemic time; e, long ischemic time. The upper half contains data for the effect of NOX2 on infarct size (IS); the lower half, on neurological score (NS). Displayed are the SMD, 95\% confidence intervals and relative weight of the individual studies. The diamond indicates the global SMD and its 95\% confidence interval. 


\section{Most studies on NOX2 in stroke were insufficiently powered}

We next analysed whether those studies that were included in the systematic review were actually sufficiently powered to detect an effect size of $40 \%$, which is considered a minimum to be clinically relevant (see Methods). Since especially in small studies the observed variance is not a precise estimate of the true variance, we computed pooled variances. For four different groups (short ischemia time, medium ischemia time, long ischemia time and long reperfusion time), we found these calculated pooled variances to be $0.44,0.60,0.29$ and 0.43 , respectively. With these and the number of animals described in each study, we calculated the power to detect a difference of at least 40\% (Table 1). Most notably, none of the studies reached a sufficient level of power $(1-\beta \geq 0.08)$, with the exception of our own earlier study $(1-ß=0.95)$, which had shown no effect for NOX2 ${ }^{24}$.

\section{Publication bias leads to an overestimation of the NOX2 effect size in stroke}

In addition to insufficient power, publication bias can also influence preclinical study results. To identify a possible publication bias for the outcome 'infarct size', we created a funnel plot ${ }^{10,49}$. Indeed, in Fig. 2 we show that it is likely that several small studies reporting larger infarcts in NOX2 KO mice are missing from the literature. This may indicate an overestimation of the overall effect (SMD -0,66 [-1,20;-0,14]) as calculated in the meta- analysis and question the significance of NOX2. Moreover, reporting of essential details of the animal stroke studies was poor and there was a significant risk of bias (SI Appendix Fig. S2). Only 31\% of the studies reported on blinding of the outcome measures. In addition, just $15 \%$ of the studies described whether they randomized the allocation of the animals to the various groups. None of the papers described the method for randomization. This is surprising as blinding and randomization are key quality measures of proper experimental design of intervention studies, and are known to cause bias ${ }^{50}$. Thus, publication bias, poor methodological quality and lack of power of all studies that found an effect for NOX2 in stroke cast considerable doubt on preclinical target validation. 
Table 1. Power analysis of studies on the role of NOX2 in experimental stroke.

\begin{tabular}{|c|c|c|c|c|c|c|c|c|c|c|c|c|}
\hline \multirow[t]{2}{*}{ Reference } & \multirow[t]{2}{*}{ Gender } & \multirow{2}{*}{$\begin{array}{l}\text { Duration } \\
\text { ischemia } \\
\text { (min) }\end{array}$} & \multirow{2}{*}{$\begin{array}{l}\text { Time of } \\
\text { sacrifice } \\
\text { (h) }\end{array}$} & \multicolumn{5}{|c|}{ NOX2 WT } & \multicolumn{3}{|c|}{ NOX2 KO } & \multirow{2}{*}{$\begin{array}{c}\text { Power } \\
\text { for } 40 \% \\
\text { effect }\end{array}$} \\
\hline & & & & $\mathrm{N}$ & Mean $\left(\mathrm{mm}^{3}\right)$ & SD & $\mathrm{CV}$ & $\mathrm{N}$ & Mean $\left(\mathrm{mm}^{3}\right)$ & SD & $\mathrm{CV}$ & \\
\hline \multicolumn{13}{|c|}{ Reperfusion time $24 \mathrm{~h}$, short ischemia } \\
\hline \multirow[t]{2}{*}{ Brait $^{36}$} & $\mathrm{M}$ & 30 & 24 & 8 & 58,63 & 32,16 & 0,55 & 10 & 30,04 & 28,37 & 0,94 & 26 \\
\hline & $\mathrm{F}$ & 30 & 24 & 7 & 24,93 & 17,04 & 0,68 & 10 & 42,69 & 17,01 & 0,40 & 24 \\
\hline De Silva ${ }^{33}$ & $M$ & 30 & 24 & 15 & 36,30 & 13,20 & 0,36 & 7 & 14,70 & 10,20 & 0,69 & 28 \\
\hline \multicolumn{13}{|c|}{ Reperfusion time $24 \mathrm{~h}$, middle long ischemia } \\
\hline Chen $^{38}$ & $\mathrm{M}$ & 75 & 24 & $5^{\star}$ & 53,90 & 19,23 & 0,36 & $5^{*}$ & 26,00 & 10,73 & 0,41 & 54 \\
\hline Chen ${ }^{39}$ & $M$ & 75 & 24 & $3^{*}$ & 50,00 & 7,79 & 0,16 & $3^{*}$ & 28,50 & 3,93 & 0,14 & 29 \\
\hline Kleinschnitz ${ }^{24}$ & $M$ & 60 & 24 & 10 & 78,70 & 19,50 & 0,25 & 18 & 79,18 & 20,90 & 0,26 & 95 \\
\hline \multirow[t]{2}{*}{ McCann ${ }^{44}$} & $M$ & 60 & 6 & 5 & 12,90 & 4,30 & 0,33 & 5 & 9,50 & 2,10 & 0,22 & 59 \\
\hline & M & 60 & 24 & 5 & 35,60 & 4,30 & 0,12 & 6 & 15,10 & 3,10 & 0,21 & 54 \\
\hline \multicolumn{13}{|c|}{ Reperfusion time $24 \mathrm{~h}$, long ischemia } \\
\hline Liu $^{43}$ & $\mathrm{M}$ & 90 & 24 & 5 & $38,00 \dagger$ & 2,00 & 0,05 & 5 & $25,00 \dagger$ & 5,00 & 0,20 & 16 \\
\hline Kahles ${ }^{35}$ & $M$ & 120 & 24 & 13 & 83,88 & 41,92 & 0,50 & 14 & 43,20 & 38,23 & 0,88 & 41 \\
\hline Tang ${ }^{34}$ & $M$ & 120 & 24 & 6 & 163,83 & 35,59 & 0,22 & 4 & 69,41 & 35,66 & 0,51 & 15 \\
\hline Walder $^{23}$ & M & 120 & 24 & 10 & 54,00 & 33,52 & 0,62 & 13 & 29,10 & 20,19 & 0,69 & 35 \\
\hline Wang ${ }^{40}$ & $\mathrm{M}$ & 120 & 24 & 8 & 35,25 & 9,50 & 0,27 & 8 & 19,38 & 8,55 & 0,44 & 25 \\
\hline \multicolumn{13}{|c|}{ Reperfusion time $72 \mathrm{~h}$} \\
\hline Chen $^{38}$ & M & 75 & 72 & $5^{*}$ & 106,20 & 27,95 & 0,26 & $5^{\star}$ & 52,00 & 26,39 & 0,51 & 25 \\
\hline Chen ${ }^{39}$ & $M$ & 75 & 72 & $3^{*}$ & 86,00 & 16,00 & 0,19 & $3^{*}$ & 55,00 & 16,16 & 0,29 & 14 \\
\hline Kunz ${ }^{37}$ & M & 25 & 72 & $5^{\star}$ & 52,00 & 24,40 & 0,47 & $5^{*}$ & 20,10 & 11,70 & 0,58 & 25 \\
\hline McCann ${ }^{44}$ & M & 90 & 72 & 5 & 28,77 & 14,14 & 0,49 & 8 & 20,92 & 9,28 & 0,44 & 31 \\
\hline
\end{tabular}


Table 1, continued

\begin{tabular}{|c|c|c|c|c|c|c|c|c|c|c|c|c|}
\hline \multicolumn{13}{|c|}{ Reperfusion time $24 \mathrm{~h}$, middle long ischemia, including current study } \\
\hline Chen ${ }^{38}$ & M & 75 & 24 & 5 * & 53,90 & 19,23 & 0,36 & 5 * & 26,00 & 10,73 & 0,41 & 48 \\
\hline Chen ${ }^{39}$ & M & 75 & 24 & 3* & 50,00 & 7,79 & 0,16 & 3* & 28,50 & 3,93 & 0,14 & 26 \\
\hline Kleinschnitz ${ }^{24}$ & M & 60 & 24 & 10 & 78,70 & 19,50 & 0,25 & 18 & 79,18 & 20,90 & 0,26 & 92 \\
\hline \multirow[t]{2}{*}{ McCann ${ }^{44}$} & $M$ & 90 & 6 & 5 & 12,90 & 4,30 & 0,33 & 5 & 9,5 & 2,10 & 0,22 & 53 \\
\hline & $\mathrm{M}$ & 90 & 24 & 5 & 35,60 & 4,30 & 0,12 & 6 & 15,10 & 3,10 & 0,21 & 48 \\
\hline \multirow[t]{3}{*}{ Multicentre } & $\mathrm{F}$ & 60 & 24 & 10 & 99,77 & 33,12 & 0,33 & 19 & 75,44 & 32,99 & 0,44 & 97 \\
\hline & $M$ & 60 & 24 & 21 & 76,76 & 10,53 & 0,14 & 14 & 84,66 & 9,31 & 0,11 & 93 \\
\hline & All & 60 & 24 & 41 & 89,00 & 32 & 0,36 & 51 & 80,00 & 31,00 & 0,39 & 100 \\
\hline
\end{tabular}

To assess the power of already published studies, original data sets were requested from the authors or when original data set not available, were extracted from the text or figures (using 'universal desk top ruler' software). Means, SD's and numbers of animals were extracted from the original data or, if original data sets could not be obtained, from the text or figures using 'universal desk top ruler' software. If only the SEM was reported, the SD was calculated using the formula $S D=\sqrt{n} \cdot S E M$. For 4 different groups, pooled variances were used to calculate the power for detecting a 40\% difference was calculated using the Russ Lenth's power and sample size software (http://homepage.stat.uiowa.edu/ rlenth/Power/index.html). The power for the 'reperfusion time 24h, middle long ischemia' groups were recalculated using the newly available data from the current multicentre study. * Lowest number of number range †Infarct size reported as percentage. 


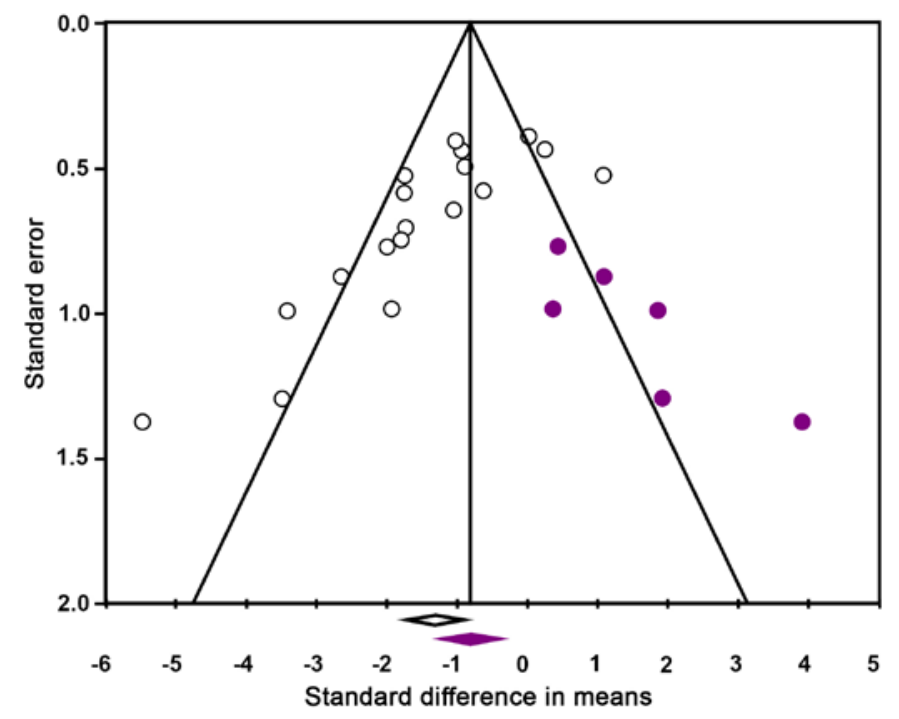

Figure 2. Funnel plot asymmetry suggesting the presence of publication bias and an overestimation of the overall effect size of NOX2 in stroke. The Y-axis represents precision, the $X$-axis effect size of individual studies. The funnel plot is based on the fact that precision in estimating the underlying treatment effect will increase as the sample size of component studies increases. Using the trim and fill analyses the intervention effect is adjusted for possible missing studies (filled purple symbols) amongst published data (open symbols). The asymmetry suggests that studies showing larger infarcts in NOX2 KO mice in experimental stroke are missing. These would otherwise shift the mean (open diamond) towards a smaller or no overall effect of NOX2 (closed purple diamond).

\section{A randomized, controlled, blinded, and fully powered pre-clinical trial excludes a relevant role for NOX2 in stroke}

To finally elucidate the relevance of NOX2 in experimental stroke, we conducted the first multi-national, randomized, controlled, and blinded animal study that was fully powered for a $40 \%$ effect size. A priori sample size calculation showed that at least 10 animals would be necessary in each study arm, which we exceeded with $n=41$ WT and $\mathrm{n}=51$ NOX2 KO. Importantly, 24h after transient middle cerebral artery occlusion (tMCAO), neither infarct distribution (Fig. 3A), infarct size (Fig. 3B), nor neurofunctional parameters such as the Bederson score (Fig. 3C) or the Grip test (Fig. 3D) were significantly different between NOX2 KO $(n=51)$ and WT $(n=41)$ mice. 
If one were to examine if the possible difference in infarct size of -10\% in NOX2 (for which our study was not powered) could become significant, a much larger study would be needed. Based on a power of $1-\beta=0.08$, and an $\alpha$ of 0,05 , a standard deviation of $30 \%$ and an effect of $10 \%$, the number of animals needed per group would be $n=142$. Taking into account an acute mortality rate of $30 \%$ (average reported) this would require the inclusion of $n=202$ animals per group. The use of 404 animals to clarify such a small and translationally insignificant effect would be ethically non-justifiable.

With respect to stroke and NOX, gender-specific effects have been reported ${ }^{51}$. In our study, a sub-group analysis showed in male NOX2 KOs a larger than average infarct size reduction of $25 \%$ (see Fig. $3 B$, second data set), which reached significance $(p=0.04)$ but was underpowered $(1-\beta=0.68)$. In any group, we did not find translationally more relevant accompanying improvements in either neurological behaviour or motor function (see Fig. $3 C$ and $D$ ). Other possible subgroups, females and young versus adult animals did also not produce any significant differences.

\section{Our revised meta-analysis suggests an even lower effect size, no neurological improvement and persisting publication bias}

To test whether these new findings would affect our above meta-analysis (see Fig. 1, left data set) we re-ran the extended data set (see Fig. 1, right data set). Still, NOX2 appeared to significantly decrease the infarct size (Fig. 1A SMD -1.15 [-1.67;-0.63]; $n=20 ; p=0.000$ ). However, the effect was now smaller and even after including our new study results, a publication bias still appeared to overestimate our overall effect estimation (SI Appendix Fig. S3). Importantly and independently of infarct size, the effect of NOX2 KO on the neurological score was no longer significant (SMD -0.37 $[-0.79 ; 0.06] ; n=12 ; p=0.094)$. Thus even if a small effect on the surrogate infarct size would ever be shown with sufficient power, we can predict it will not translate into any neurological outcome improvement. Clearly, this conclusion is a definitive counterargument against any further clinical development of this target. 


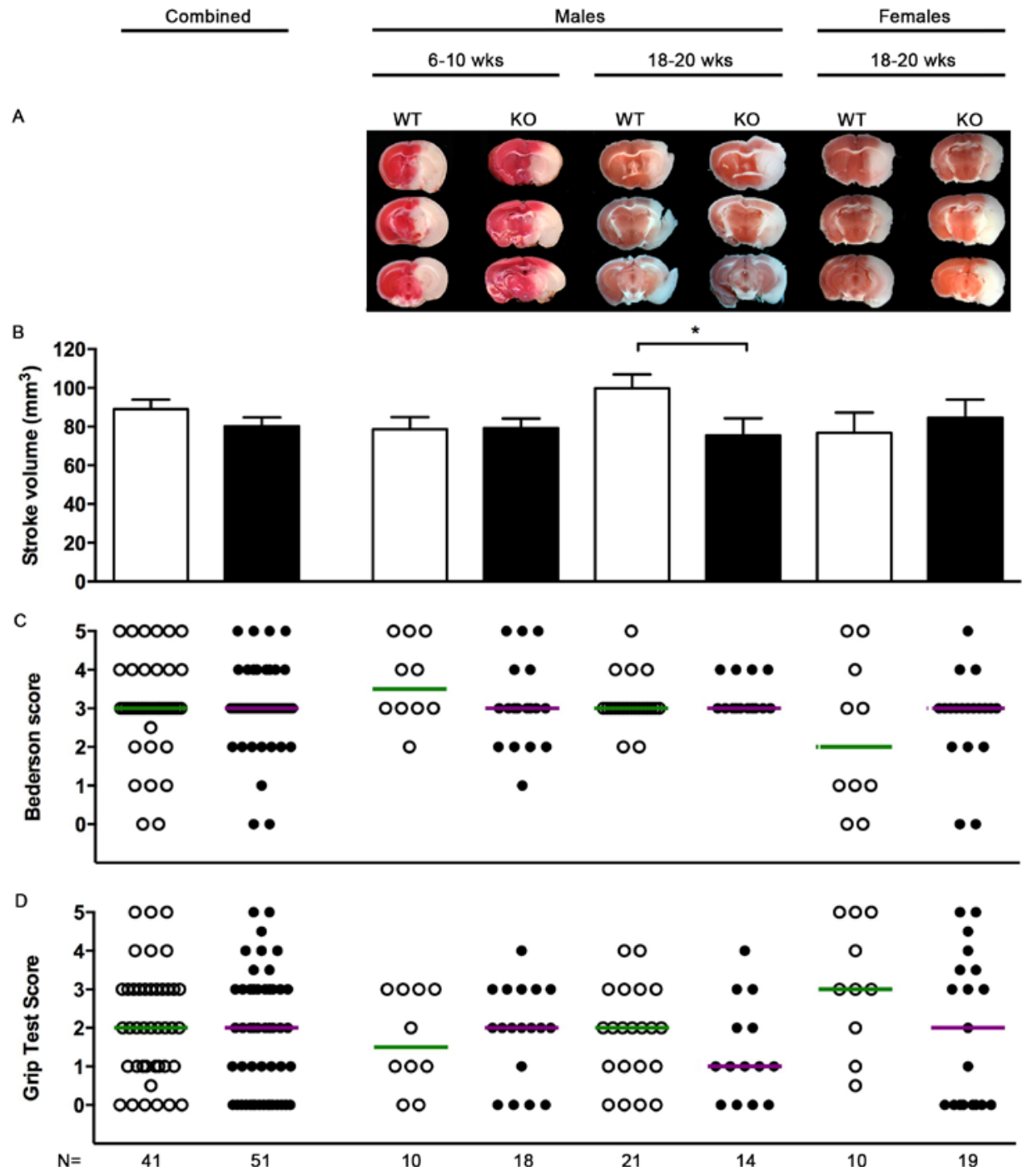

Figure 3. Infarct size and neurological function in NOX2 KO and WT mice. Mice of both genders and between 6-20 weeks old were subjected to tMCAO. A) TTC stainings of three sequential coronal brain sections on day 1 after $T M C A O$ that were representative for each subgroup: young (6-10 week old), adult (18-20 week old) male, and adult female NOX2 KO and WT mice. The TTC staining colours viable tissue red, while infarcted tissue stays white. B) Bar graphs of mean infarct volumes \pm SEM from WT (white bar) and NOX2 KO (black bars). C) Scatter plot and median of neurodeficit Bederson scores on day 1 after tMCAO, ranging from 0 (normal) to 5 (severe), from WT (open symbols, green line) and NOX2 KO mice (closed symbols, purple line). D) Scatter plot and median of grip test scores, ranging from 0 (severe deficit) to 5 (normal) from WT (open symbols, green line) and NOX2 KO mice (closed symbols, purple line). The asterisk, *, indicates statistical significance $(p<0.05)$ for the infarct size in the subgroup adult male NOX2 versus WT; however, this group was insufficiently powered to allow the detection of a difference. Combined analysis did not show a significant difference for any of the parameters. 


\section{Discussion}

Here we have elucidated in great depth one major problem of current biomedical research, the irreproducibility of pre-clinical results leading subsequently to translational failures towards the path to the clinic. We chose to examine stroke, an area of extreme medical need and probably one of the lowest translational success rates in biomedicine, and the possible role of NOX2 as neuroprotective target in ischemic stroke. However, we feel confident that what we show applies most likely to many other fields and many other claimed pharmacological targets.

A profound body of pre-clinical literature seemed to suggest that NOX2 is a therapeutic target in stroke. Surprisingly, our own initial data had argued against this. We thus conducted a systematic review after contacting every group that had published in this field on this pharmacological target. Indeed our subsequent metaanalysis suggested that a small effect of NOX2 on infarct size and neurological score may exist. However, we show that there was also a significant publication bias in the literature that had lead to an overestimation of the overall effect size. In the present case, complete reporting of data would have shifted the true effect size towards a lower or no role of NOX2 in stroke. Of worry was also that reporting of essential experimental parameters was rather poor. Failures in reporting these details skew the interpretation of study results and subsequent translation into clinical benefits. If important methodological issues such as randomization and blinding are neglected the reliability of basic research is affected and may cause an overestimation of the efficacy of experimental interventions. Another crucial flaw was the lack of statistical power to detect a relevant infarct size reduction of $40 \%$ in all studies that reported significant effects. Collectively, several underpowered studies can contribute to a reliable, i.e. powered, effect size estimation. However, better randomization, blinding and more standardized experimental protocols are needed to enhance the quality of preclinical experimental stroke studies and likelihood for later translational success into the clinic. 
One way forward, as we show here, is to define a minimal effect size as an obligatory outcome parameter and ensure that based on variability and number of animals the subsequent study is sufficiently powered to detect such an effect. With respect to stroke, a pre-clinical effect size of under $40 \%{ }^{52}$ was later still insufficient to ensure clinical benefit ${ }^{53-55}$; in one case the outcome was even worse ${ }^{56}$. Thus our chosen minimal relevant effect size of $40 \%$ was a rather mild threshold. The true threshold is likely to be higher, but in the absence of any successful translation of pre-clinical stroke research in the past 20 years ${ }^{16}$, this cannot be determined. Ideally, experimental animal work for target validation will follow in future the guidelines and quality criteria that have been successfully established for clinical research, as has recently been proposed also by Boltze et al ${ }^{21}$.

We here present the first preclinical randomized controlled trial (pRCT) approach to validate a single intervention or in this case target validation. Based on its outcome data, a NOX2 KO does not improve neurological outcome and has an effect on infarct size that was too small to be determined up by a trial powered for $40 \%$ reduction. Conducting another PRCT using $>400$ animals would be required to determine whether indeed a 10\% infarct size reduction occurs; this however would be translationally irrelevant (see above examples and threshold) and thus unethical. Moving to pRCTs as the new quality standard, at least for pharmacological target validation, will in all likelihood exceed individual laboratories' capacity. Thus conducting such studies in a more collaborative manner, meaning multi-centre trials, seems to be the logical way forward. In fact, for other targets, including very late antigen 4 (VLA-4) ${ }^{57}$ and transient receptor potential cation channel, subfamily $\mathrm{M}$, member 2 (TRPM2; CK, unpublished), such trials are currently under way already. A website has been launched (www.p3pt.de) as an invitation to the community to provide their position on pRCTs and potential suggestions how those should be organized and performed ${ }^{21}$.

Importantly, implications reach further. The studies that we have analysed were conducted in Korea, Germany, USA, Australia, and the Netherlands. 
Animal ethics regulations differ, but at least for the European Community it can be said that in recent years there has been a massive push towards 'The Three Rs', reduction, replacement, and refinement ${ }^{58}$. However, reducing the number of animals below the limits of statistical power will lead to underpowered and in the end meaningless pre-clinical data sets. Whilst we strongly support the goal to achieve pre-clinical evidence with the least amount of animal sacrifice, the use and reporting of a power calculation is essential to the proper conduct of animal studies. Instead, we here present a strategy to reduce translational failure by maximizing the use of systematic reviews, then conducting a fully powered pRCT and finally re-analysing by subsequent meta-analysis.

Finally, we identified a persistent publication bias, i.e. even after including our own new PRCT data. This suggests at least two reactions. Journals need to equally accept for publication well-conducted (e.g. statistically powered) negative findings, so that the literature is truly representative of the science. To ensure that such findings are accessible, even if not submitted for publication, a rather far-reaching but effective measure would be to require pre-registration of animal experiments, whether they were conducted as pRCTs or just pilots, similar to requirements for clinical trials. Registration would be a pre-requisite for ethics approval and include the obligation to subsequently enter the data into a publicly available database. This will enable to conduct at least meta-analyses as a surrogate for pRCTs to combine small animal studies in a meaningful manner.

\section{Material and methods}

\section{Animals}

NOX2 deficient mice (NOX2 KO, stock \#002365) from C57BI/6J background and corresponding age-matched C57BI/6J control mice (stock \#000664) were purchased from Jackson Laboratories (Bar Harbor, ME, USA). In a previous study ${ }^{24}$ we already tested young male (6-8 weeks, 20-25 grams) mice. We therefore extended our inclusion criteria by using also female (8-10 weeks, 18-21 grams) and also older (18-20 weeks, 26-31 grams) mice. All experiments were approved by the local animal ethics committees (Maastricht DEC 2011-106, Würzburg ethical approval 69/08). 
Animals were socially housed in IVC cages under controlled conditions $\left(22^{\circ} \mathrm{C}\right.$, $55-65 \%$ humidity, $12 \mathrm{~h}$ light-dark cycle), and were allowed free access to water and standard laboratory chow.

\section{Systematic review and meta-analysis}

The present review was based on published results of animal studies on the role of NADPH oxidase 1 and/or 2 in experimental ischemic stroke. PubMed and EMBASE were searched for original papers and conference abstracts concerning the effects of NADPH oxidase 1 and/or 2 on experimental stroke until October 23, 2013. The search strategy involved the following 3 search components: ischemic stroke, NADPH oxidase 1 and/or 2 and animals (for the complete search strategy, see Table S1). For detecting animal studies, search filters developed by SYRCLE were used ${ }^{59,60}$. No language restriction was used. Two independent researchers (PWMK and SSJR) screened all titles and abstracts for the inclusion criteria. Studies were included if they 1) investigated the role of NADPH oxidase 1 and/or 2 on the infarct size and neurological scoring after experimental focal ischemic stroke using either genetic or specific pharmacological inhibition of these NOX isoforms; 2) were performed in animals in vivo; 3) resulted in an original full paper which presented unique data. Papers were excluded when unspecific NOX inhibitors such as apocynin ${ }^{61,62}$ were used or when NOX inhibition was combined with other drugs/therapies. The in- and exclusion criteria and methods of analysis were specified in advance and documented in a protocol.

\section{Study characteristics and data extraction}

From the included studies, bibliographic data such as authors, year of publication, journal of publication and language were registered. Study characteristics concerning study design were extracted and summarized in SI Appendix Table S2: species, strain (including genetic KO), gender, age and weight of the animals used; type of anaesthesia; method and duration of ischemia; duration of reperfusion (timing of outcome measurements), type of inhibitor used; method of culling; method of infarct size measurement and neurological outcome assessment; (reason for) dropouts and mortality. Infarct size and neurological outcome were included in the meta-analysis. 
Data were extracted if raw data or group averages, standard deviation (SD) or standard error (SE), and number of animals per group (n) were reported, or could be recalculated. All authors were contacted to contribute their original data to the metaanalysis. From 2 publications, no response from authors was obtained. For one publication, authors could not recollect their original data. In these cases, data were extracted from the text, or if presented only graphically, measured using a universal on-screen digitizer (Universal Desktop Ruler). Study quality and risk of bias in the included studies was independently assessed by two reviewers, using a predefined 9-point rating system (based on ${ }^{50}$, see Table S3 and legend for details). Seven items were assessed to study risk of bias. A "yes" judgement indicates a low risk of bias; a "no" judgment indicates high risk of bias; the judgment will be "unclear" if insufficient details have been reported to assess the risk of bias properly. Because of poor reporting of essential details in animal studies, we also included 2 reporting item: we assessed whether it was reported if the experiments were randomized or blinded at any level (point 1 and 3). Disagreements were solved by discussion.

\section{Data synthesis and statistical analysis}

For both the outcome measure 'infarct size' and 'neurological score' the standardized mean difference (SMD) was calculated. An SMD expresses the difference between the groups relative to the standard deviation. Calculation of mean differences ${ }^{46-48}$ was not possible because of the heterogeneity in study designs (i.e. species) and the variety of scales used to determine the outcomes. The studies of NOX1 and NOX2 were analysed separately. In case different measures of neurobehavioral outcomes were reported from the same cohort of animals we pooled the individual effect sizes and used this pooled estimate in the overall meta-analysis. Despite anticipated heterogeneity, the individual SMDs were pooled whenever possible (starting from two studies or more) to obtain an overall SMD and 95\% confidence interval.

To account for anticipated heterogeneity, we used the random effects model in which some heterogeneity beyond sampling errors is allowed. In order to assess the robustness of our findings and in an attempt to explain observed study heterogeneity, we performed a sensitivity analysis and we investigated the effects of excluding the study with permanent ischemia. 
Meta-analysis was performed using Comprehensive Meta Analysis (CMA version 2.0). Forest plots were used to display the mean overall effect sizes. Data are expressed as SMD with 95\% confidence intervals. For the outcome measure infarct size, we assessed the possibility of publication bias by visually evaluating the possible asymmetry in funnel plots ${ }^{63}$. Using the trim and fill analyses an adjusted intervention effect was calculated ${ }^{49}$.

\section{pRCT}

The preclinical Randomized, Controlled (and blinded) animal Trial was performed in parallel at Maastricht University (The Netherlands) and at the University of Würzburg (Germany). The objective of the study was to compare the extent of neurological damage after stroke in mice with or without NOX2 gene deletion. At each study site, surgery and follow-up measurements were performed blinded and animals were operated randomly according to an online randomization tool (www.randomizer.org). For a power of $80 \%$, based on a minimal effect on infarct size of $-40 \%$ and an SD of $30 \%$, the required animal numbers were at least $n=10$ per study arm. Transient middle cerebral artery occlusion (tMCAO) was performed with an intraluminal filament method as described by Kleinschnitz et al. ${ }^{24}$. After 60 minutes of ischemia, the filament was withdrawn and reperfusion established. Twenty-four hours after induction of the ischemia, mice were scored for neurological and motor function. Infarct size was determined using 2, 3, 5-triphenyltetrazolium chloride (TTC) staining. For a more detailed description, see the SI Appendix Methods.

\section{Power analysis}

We post hoc analysed the power of earlier published studies on NOX2 in stroke. Twelve studies and our own new data were analysed for their power to detect a difference of $40 \%$ in infarct size. This threshold of $40 \%$ difference was based on posthoc analysis of failed clinical trials where preclinical studies showed a $30-40 \%$ difference ${ }^{29,52,64}$. Power was calculated using Russ Lenth's power software, an alpha of 0,05, an effect of 0.4 and a pooled variance [(Lenth, R.V 2006-9, java Applets for Power and Sample Size [Computer Software], Retrieved 02-17-2014, from http://www.stat.uiowa.edu/ rlenth/Power)]. 
The pooled variance was calculated for 4 different groups according to ischemic and reperfusion time: a) long reperfusion time $(72 \mathrm{~h}), \mathrm{b})$ short reperfusion time (24h) after short ischemia, c) short reperfusion time (24h) after medium ischemia, and d) short reperfusion time (24h) after long ischemia. From each individual study, the coefficient of variation was calculated for both KO and WT values. Taken into account all these values, the pooled variance for each group was calculated according to the formula $S_{p}=\sqrt{ }\left(\frac{\Sigma\left(c V^{2} \cdot(n-1)\right)}{\Sigma(n-1)}\right)$ with $\mathrm{n}$ the size of the group and $\mathrm{CV}$ the coefficient of variation (SD/Mean) of the group.

\section{Statistics}

Infarct volume data are expressed as mean \pm SEM. Statistical differences between mean values were determined by Student's two-tailed $t$ test, using the GraphPad Prism 5.0 software package. Neurological scores were expressed as median. For discrete variables (behavior and motor function scores), the Mann-Whitney U-test was used. A value of $p<0.05$ was considered to be statistically significant. Power calculations were performed by using Russ Lenth's power and sample size software.

\section{ACKNOWLEDGMENTS}

We are greatly thankful to all colleagues and fellow-researchers who were willing to dig deep in their records and share their data sets with us. We also thank Dr. Emily Sena from CAMARADES for most helpful discussions. This work was supported by a Marie-Curie International Reintegration Grant (IRG), an ERC Advanced Investigator Grant, by the Nederlandse Hersenstichting, by the NHMRC Australia (awarded to HHHWS), and by the Deutsche Forschungsgemeinschaft (awarded to CK). We also gratefully acknowledge Radboud University Nijmegen for statistical advice and Helma van Essen and Jacques Debets, Maastricht University, for their expert technical assistance in the in vivo experiments. 


\section{References}

1. Loscalzo, J. Irreproducible experimental results: causes, (mis)interpretations, and consequences. Circulation 125, 1211-1214 (2012).

2. Prinz, F., Schlange, T. \& Asadullah, K. Believe it or not: how much can we rely on published data on potential drug targets? Nat Rev Drug Discov 10, 712-712 (2011).

3. Anonymous. Facilitating reproducibility. Nat. Chem. Biol. 9, 345-345 (2013).

4. Mullard, A. Reliability of 'new drug target' claims called into question. Nat Rev Drug Discov 10, 643-644 (2011).

5. Arrowsmith, J. Trial watch: Phase II failures: 2008-2010. Nat Rev Drug Discov 10, 328-329 (2011).

6. Seok, J. et al. Genomic responses in mouse models poorly mimic human inflammatory diseases. Proc Natl Acad Sci U S A 110, 3507-3512 (2013).

7. van der Worp, H. B. et al. Can animal models of disease reliably inform human studies? PLoS Med. 7, e1000245 (2010).

8. Dirnagl, U. \& Fisher, M. International, multicenter randomized preclinical trials in translational stroke research: it's time to act. J Cereb Blood Flow Metab 32, 933-935 (2012).

9. Sterne, J. A. \& Davey Smith, G. Sifting the evidence-what's wrong with significance tests? BMJ 322, 226-231 (2001).

10. Sena, E. S., van der Worp, H. B., Bath, P. M. W., Howells, D. W. \& Macleod, M. R. Publication bias in reports of animal stroke studies leads to major overstatement of efficacy. PLoS Biol. 8, e1000344 (2010).

11. Kilkenny, C. et al. Survey of the quality of experimental design, statistical analysis and reporting of research using animals. PLOS ONE 4, e7824 (2009).

12. Eisen, J. A., Ganley, E. \& MacCallum, C. J. Open science and reporting animal studies: who's accountable? PLoS Biol. 12, e1001757 (2014).

13. Philip, M., Benatar, M., Fisher, M. \& Savitz, S. I. Methodological quality of animal studies of neuroprotective agents currently in phase II/III acute ischemic stroke trials. Stroke 40, 577-581 (2009). 
14. Maiser, S. J. et al. Intravenous recombinant tissue plasminogen activator administered after $3 \mathrm{~h}$ following onset of ischaemic stroke: a metaanalysis. Int $\mathrm{J}$ Stroke 6, 25-32 (2011).

15. Radermacher, K. A. et al. The 1027th target candidate in stroke: Will NADPH oxidase hold up? Exp Transl Stroke Med 4, 11 (2012).

16. O'Collins, V. E. et al. 1,026 experimental treatments in acute stroke. Ann. Neurol. 59, 467-477 (2006).

17. Fisher, M. et al. Update of the stroke therapy academic industry roundtable preclinical recommendations. Stroke 40, 2244-2250 (2009).

18. Stroke Therapy Academic Industry Roundtable (STAIR). Recommendations for standards regarding preclinical neuroprotective and restorative drug development. Stroke 30, 2752-2758 (1999).

19. O'Collins, V. E. et al. Preclinical drug evaluation for combination therapy in acute stroke using systematic review, meta-analysis, and subsequent experimental testing. J Cereb Blood Flow Metab 31, 962-975 (2011).

20. Dirnagl, $U$. Bench to bedside: the quest for quality in experimental stroke research. J Cereb Blood Flow Metab 26, 1465-1478 (2006).

21. Boltze, J., Ayata, C., Wagner, D.-C. \& Plesnila, N. Preclinical phase III trials in translational stroke research: call for collective design of framework and guidelines. Stroke 45, 357-357 (2014).

22. Tymianski, M. Novel approaches to neuroprotection trials in acute ischemic stroke. Stroke 44, 2942-2950 (2013).

23. Walder, C. E. et al. Ischemic stroke injury is reduced in mice lacking a functional NADPH oxidase. Stroke 28, 2252-2258 (1997).

24. Kleinschnitz, C. et al. Post-stroke inhibition of induced NADPH oxidase type 4 prevents oxidative stress and neurodegeneration. PLOS Biol. 8, (2010).

25. Chen, H. et al. Oxidative stress in ischemic brain damage: mechanisms of cell death and potential molecular targets for neuroprotection. Antioxid Redox Signal 14, 1505-1517 (2011).

26. Schroder, K. et al. Nox4 is a protective reactive oxygen species generating vascular NADPH oxidase. Circ Res 110, 1217-1225 (2012). 
27. Schmidt, H. H. H. W., Wingler, K., Kleinschnitz, C. \& Dusting, G. NOX4 is a Janus-faced reactive oxygen species generating NADPH oxidase. Circ Res 111, e15-6- author reply e17-8 (2012).

28. Shuaib, A. et al. NXY-059 for the treatment of acute ischemic stroke. N. Engl. J. Med. 357, 562-571 (2007).

29. Macleod, M. R. et al. Evidence for the efficacy of NXY-059 in experimental focal cerebral ischaemia is confounded by study quality. Stroke 39, 2824-2829 (2008).

30. Radermacher, K. A. et al. Neuroprotection after stroke by targeting NOX4 as a source of oxidative stress. Antioxidants and Redox Signaling 18, 1418-1427 (2013).

31. Sedeek, M. et al. Renoprotective effects of a novel Nox1/4 inhibitor in a mouse model of type 2 diabetes. Clin. Sci. (2012). doi:10.1042/CS20120330

32. Marco, E. et al. Pharmacological inhibition of NOX reduces atherosclerotic lesions, vascular ROS and immune-inflammatory responses in diabetic Apoe -/- mice. Diabetologia (2013). doi:10.1007/s00125-013-3118-3

33. De Silva, T. M., Brait, V. H., Drummond, G. R., Sobey, C. G. \& Miller, A. A. Nox2 oxidase activity accounts for the oxidative stress and vasomotor dysfunction in mouse cerebral arteries following ischemic stroke. PLoS ONE 6, e28393 (2011)

34. Tang, X. N., Zheng, Z., Giffard, R. G. \& Yenari, M. A. Significance of marrowderived nicotinamide adenine dinucleotide phosphate oxidase in experimental ischemic stroke. Ann. Neurol. 70, 606-615 (2011).

35. Kahles, T. et al. NADPH oxidase plays a central role in blood-brain barrier damage in experimental stroke. Stroke 38, 3000-3006 (2007).

36. Brait, V. H. et al. Mechanisms contributing to cerebral infarct size after stroke: gender, reperfusion, T lymphocytes, and Nox2-derived superoxide. J Cereb Blood Flow Metab 30, 1306-1317 (2010).

37. Kunz, A., Anrather, J., Zhou, P., Orio, M. \& Iadecola, C. Cyclooxygenase-2 does not contribute to postischemic production of reactive oxygen species. $J$ Cereb Blood Flow Metab 27, 545-551 (2007). 
38. Chen, H., Song, Y. S. \& Chan, P. H. Inhibition of NADPH oxidase is neuroprotective after ischemia-reperfusion. J Cereb Blood Flow Metab 29, 1262-1272 (2009).

39. Chen, H., Kim, G. S., Okami, N., Narasimhan, P. \& Chan, P. H. NADPH oxidase is involved in post-ischemic brain inflammation. Neurobiol Dis 42, 341348 (2011).

40. Wang, Z. et al. NOX2 deficiency ameliorates cerebral injury through reduction of complexin II-mediated glutamate excitotoxicity in experimental stroke. Free Radic Biol Med 65, 942-951 (2013).

41. Gray, S. P. et al. NADPH oxidase 1 plays a key role in diabetes mellitusaccelerated atherosclerosis. Circulation 127, 1888-1902 (2013).

42. Kim, H. A. et al. Brain infarct volume after permanent focal ischemia is not dependent on Nox2 expression. Brain Res. 1483, 105-111 (2012).

43. Liu, W. et al. Normobaric hyperoxia inhibits NADPH oxidase-mediated matrix metalloproteinase-9 induction in cerebral microvessels in experimental stroke. J. Neurochem. 107, 1196-1205 (2008).

44. McCann, S., DUSTING, G. \& Roulston, C. 'Time-dependent superoxide generation in NOX2 knockout mice following middle cerebral artery occlusion and reperfusion' in Abstracts of the 6th International Symposium on Neuroprotection and Neurorepair. Rostock, Germany. October 1-4, 2010. (2010).

45. Kim, H. A. et al. Brain infarct volume after permanent focal ischemia is not dependent on Nox2 expression. Brain Res. 1483, 105-111 (2012).

46. Vesterinen, H. M. et al. Meta-analysis of data from animal studies: a practical guide. J. Neurosci. Methods 221, 92-102 (2014).

47. Tsilidis, K. K. et al. Evaluation of excess significance bias in animal studies of neurological diseases. PLoS Biol. 11, e1001609 (2013).

48. Sena, E. S., Currie, G. L., McCann, S. K., Macleod, M. R. \& Howells, D. W. Systematic reviews and meta-analysis of preclinical studies: why perform them and how to appraise them critically. J Cereb Blood Flow Metab 34, 737-742 (2014). 
49. Duval, S. \& Tweedie, R. Trim and fill: A simple funnel-plot-based method of testing and adjusting for publication bias in meta-analysis. Biometrics 56, 455463 (2000).

50. Hooijmans, C. R. et al. SYRCLE's risk of bias tool for animal studies. BMC Med Res Methodol 14, 43 (2014).

51. Miller, A. A., Drummond, G. R., Mast, A. E., Schmidt, H. H. \& Sobey, C. G. Effect of gender on NADPH-oxidase activity, expression, and function in the cerebral circulation: role of estrogen. Stroke 38, 2142-2149 (2007).

52. Minnerup, J. et al. Meta-analysis of the efficacy of granulocyte-colony stimulating factor in animal models of focal cerebral ischemia. Stroke 39, 1855-1861 (2008).

53. Ringelstein, E. B. et al. Granulocyte colony-stimulating factor in patients with acute ischemic stroke: results of the AX200 for Ischemic Stroke trial. Stroke 44, 2681-2687 (2013).

54. Wagner, D.-C. et al. Allometric dose retranslation unveiled substantial immunological side effects of granulocyte colony-stimulating factor after stroke. Stroke 45, 623-626 (2014).

55. Pfeffer, M. A. et al. A trial of darbepoetin alfa in type 2 diabetes and chronic kidney disease. N. Engl. J. Med. 361, 2019-2032 (2009).

56. Skali, H. et al. Stroke in patients with type 2 diabetes mellitus, chronic kidney disease, and anemia treated with Darbepoetin Alfa: the trial to reduce cardiovascular events with Aranesp therapy (TREAT) experience. Circulation 124, 2903-2908 (2011).

57. Schäbitz, W.-R. \& Dirnagl, U. Are we ready to translate T-cell transmigration in stroke? Stroke 45, 1610-1611 (2014).

58. Balls, M. et al. The three Rs: the way forward: the report and recommendations of ECVAM Workshop 11. Altern Lab Anim 23, 838-866 (1995).

59. Hooijmans, C. R., Tillema, A., Leenaars, M. \& Ritskes-Hoitinga, M. Enhancing search efficiency by means of a search filter for finding all studies on animal experimentation in PubMed. Lab. Anim. 44, 170-175 (2010).

60. de Vries, R. B. M., Hooijmans, C. R., Tillema, A., Leenaars, M. \& RitskesHoitinga, M. A search filter for increasing the retrieval of animal studies in Embase. Lab. Anim. 45, 268-270 (2011). 
61. Wind, S. et al. Comparative pharmacology of chemically distinct NADPH oxidase inhibitors. Br J Pharmacol 161, 885-898 (2010).

62. Williams, H. C. \& Griendling, K. K. NADPH oxidase inhibitors: new antihypertensive agents? J. Cardiovasc. Pharmacol. 50, 9-16 (2007).

63. Egger, M., Davey Smith, G., Schneider, M. \& Minder, C. Bias in meta-analysis detected by a simple, graphical test. BMJ 315, 629-634 (1997).

64. Crossley, N. A. et al. Empirical evidence of bias in the design of experimental stroke studies: a metaepidemiologic approach. Stroke 39, 929-934 (2008).

\begin{abstract}
Abbreviations
$\mathrm{CV}$, coefficient of variation; IS, infarct score; KO, knock-out; NADPH, nicotinamide adenine dinucleotide phosphate; NOX, NADPH oxidase; NS, neuroscore; pRCT, preclinical randomized, controlled trial; ROS, reactive oxygen species; rt-PA, recombinant tissue plasminogen activator; SD, standard deviation; SE, standard error; SEM, standard error of mean; SMD, standardized mean difference; STAIR, stroke therapy academic industry round table; tMCAO, transient Middle Cerebral Artery Occlusion; TRPM2, transient receptor potential cation channel, subfamily $M$, member 2; TTC, 2,3,5- triphenyltetrazolium chloride; VLA4, very late antigen 4; WT, wild type.
\end{abstract}




\section{Supporting Information}

\section{SI Results}

Poor reporting and risk of bias in studies on NOX2 in stroke

To assess the quality of the included papers, we conducted a risk of bias analysis. However, as a result of poor reporting in most animal studies, we also assessed a few reporting criteria (item 1 and 3). SI Appendix Fig. S2 shows this risk of bias results for the NOX2 studies. Only a single study reported randomization. Also, in only $15 \%$ of the studies it was clear that at baseline groups were similar with respect to age, weight, and supplier. None of the papers mentioned whether or not they housed the animals randomly and used a random order to assess the outcomes. Only 4 papers reported blinding at any level, for 2 of these 4 studies, both the treatment and assessment, for the other 2 only the outcome assessment was blinded. Overall, the risk of bias analysis showed that reporting essential details of the animal stroke studies is poor and there seems to be a substantial risk of bias.

\section{Systematic review and meta-analysis on NOX1 in stroke}

Risk of bias analysis showed the same poor reporting as with NOX2 studies (SI Appendix Fig. S4). An overall effect on the infarct size, favouring deletion of NOX1 was found when including all studies (SI Appendix Fig. S5A; SMD -0.39 [$0.74 ;-0.04] ; n=4 ; p=0.031)$. This effect is very small and has thus no clinical relevance. In addition, the number of studies is very small, making it difficult to interpret these results. Sensitivity analysis showed that deleting the study in which rats were used, abolished the found effect. Thus, when only comparing NOX1 KO vs. NOX1 WT mice, no effect on infarct size was found (SMD -0.31 [-0.66;0.03]; n=3; $p=0.075)$. Heterogeneity between the mice studies was low $\left(I^{2}=5 \%\right)$. Neurological scorings were also not found to be different between NOX1 KO and NOX1 WT mice (SI Appendix Fig. S5B SMD -0.25 [-1.10;0.61]; n=3, p=0.574). 


\section{SI figures}

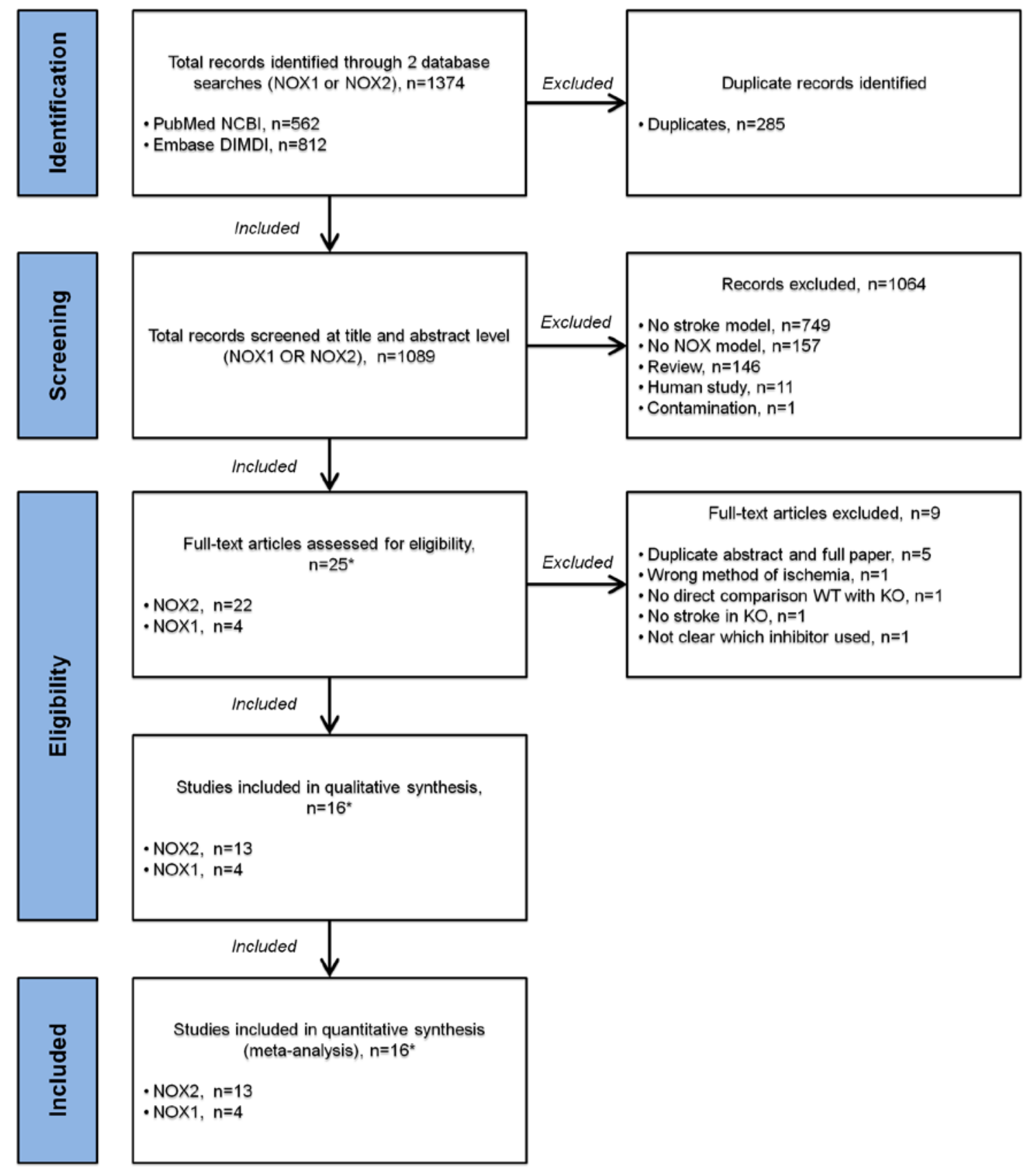

Figure S1. Flow diagram showing the different stages of the systematic review and metaanalysis on the possible role of NOX2 in stroke. Stages include identification (search of papers using Pubmed and Embase), screening (first stage eligibility screening based on title and abstract), eligibility (full text screening, using in- and exclusion criteria), and inclusion (studies included in quantitative and qualitative analysis). Exclusion criteria are listed on the right. 


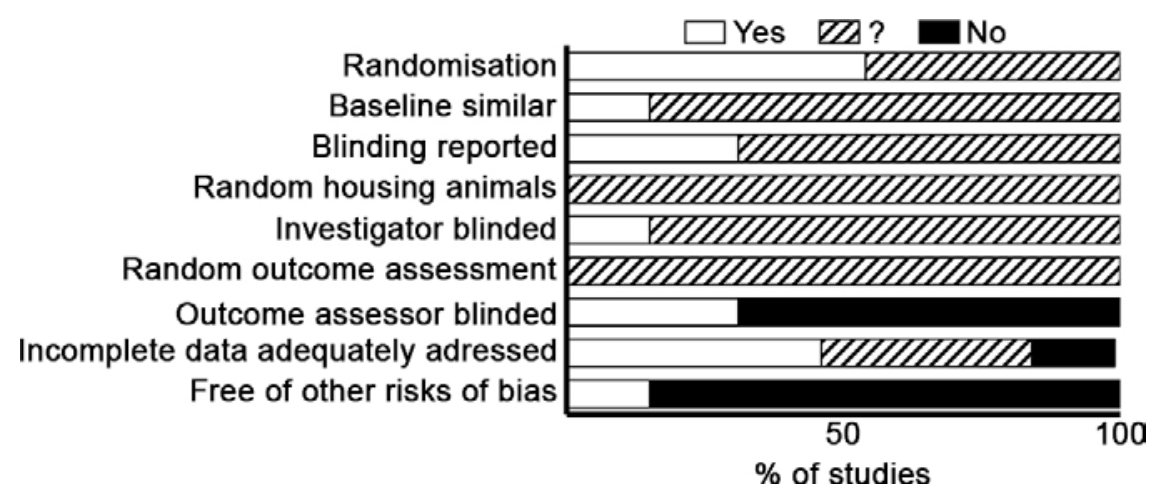

Figure S2. Risk of bias and reporting quality of NOX2 studies, averaged per item. Item, i.e. risks of bias are listed on the left. Open bars indicate a low risk of bias; closed, a high risk; hatched bars, an unclear risk. Items 1 and 3 scored reporting.

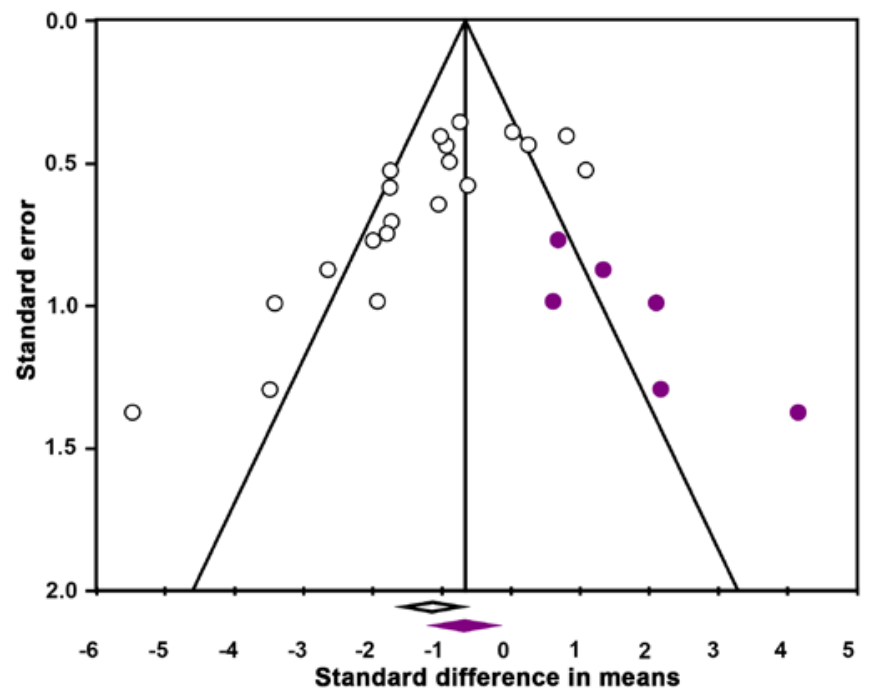

Figure S3. Updated funnel plot with the data from the current trial. Funnel plot asymmetry still suggests the presence of publication bias and an overestimation of the overall effect size of NOX2 in stroke. Using the trim and fill analyses the intervention effect is adjusted for possible missing studies (filled purple symbols) amongst published and present data (open symbols). Studies showing larger infarcts in NOX2 KO mice and thus a smaller or no effect of NOX2 in experimental stroke are still missing. For further explanations see Fig. 2. 


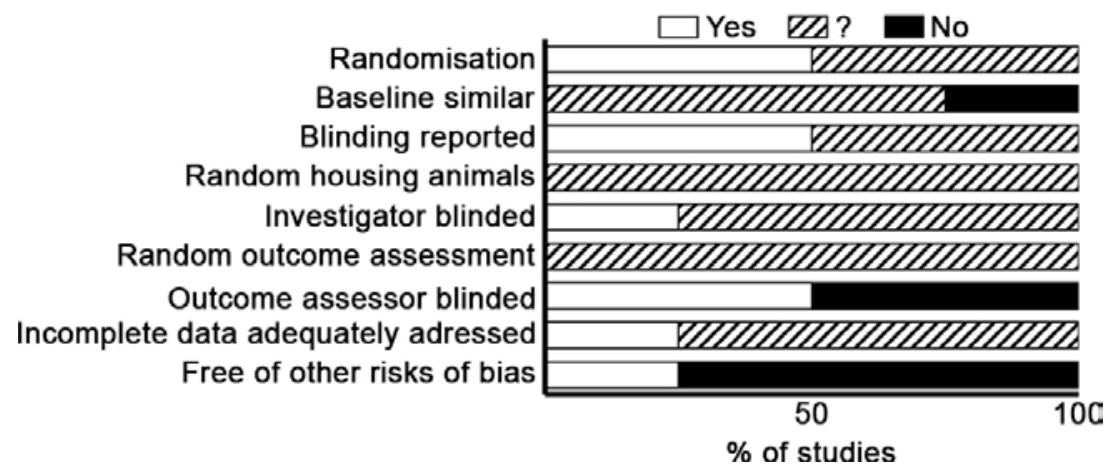

Figure S4. Risk of bias and reporting quality of NOX1 studies. For further explanation refer to Fig. S2.

\begin{tabular}{|c|c|c|c|c|c|c|c|c|c|}
\hline \multirow{2}{*}{$\frac{\text { Infarct size (IS) NOX2 }}{\text { A }}$} & \multicolumn{4}{|c|}{ Statistics for each study } & \multicolumn{5}{|c|}{ Std diff in means and $95 \% \mathrm{Cl}$} \\
\hline & $\begin{array}{l}\text { Std ditt } \\
\text { in means }\end{array}$ & $\begin{array}{l}\text { Lower } \\
\text { limit }\end{array}$ & $\begin{array}{l}\text { Upper } \\
\text { limit }\end{array}$ & p-Value & & Decreased & & Increas & \\
\hline Choi 2010 & -1.05 & -2.10 & -0.01 & 0.048 & & & & & \\
\hline Jackman 2009 & 0.07 & -0.64 & 0.79 & 0.842 & & & & & \\
\hline Kahles 2011 a & -1.00 & -1.83 & -0.16 & 0.019 & & & & & \\
\hline Kahles 2011 b & -0.40 & -0.99 & 0.18 & 0.174 & & & & & \\
\hline Kahles 2011 c & -0.08 & -0.94 & 0.78 & 0.853 & & & & & \\
\hline \multirow[t]{3}{*}{ Kleinschnitz 2010} & -0.16 & -1.09 & 0.77 & 0.741 & & & & & \\
\hline & -0.39 & -0.74 & -0.04 & 0.031 & & & & & † \\
\hline & & & & & -8.00 & -4.00 & 0.00 & 4.00 & 8.00 \\
\hline \multirow[t]{2}{*}{ Neuroscore (NS) NOX2 } & \multicolumn{3}{|c|}{ Statistics for each study } & & \multicolumn{5}{|c|}{ Std diff in means and $95 \% \mathrm{Cl}$} \\
\hline & $\begin{array}{l}\text { Std diff } \\
\text { in means }\end{array}$ & $\begin{array}{l}\text { Lower } \\
\text { limit }\end{array}$ & $\begin{array}{l}\text { Upper } \\
\text { limit }\end{array}$ & p-Value & & Increased & & Decreas & \\
\hline Jackman 2009 & 0.66 & -0.22 & 1.54 & 0.143 & & & & & \\
\hline Kahles 2011 & -0.80 & -1.58 & -0.01 & 0.047 & & & & & \\
\hline \multirow[t]{3}{*}{ Kleinschnitz 2010} & -0.54 & -1.36 & 0.28 & 0.195 & & & & & \\
\hline & -0.25 & -1.10 & 0.61 & 0.574 & & & & & \\
\hline & & & & & -8.00 & -4.00 & 0.00 & 4.00 & 8.00 \\
\hline
\end{tabular}

Figure. S5. Meta-analysis of the overall effect of NOX1 on infarct size and neurological score in experimental stroke. Studies included are shown on the left and analysed by forest plots on the right. Subgroups within one study are depicted separately with the following coding: a, female gender; $b$, male gender; $c$, short ischemic time. The upper half contains data for the effect of NOX1 on infarct size (IS); the lower half, on neurological score (NS). Displayed are the SMD, 95\% confidence intervals and relative weight of the individual studies. The diamond indicates the global SMD and its 95\% confidence interval. NOX1 has no effect. 


\section{SI tables}

Table S1. Search strategy systematic review.

\begin{tabular}{|c|c|c|}
\hline Database & $\begin{array}{l}\text { Search } \\
\text { component }\end{array}$ & Search term \\
\hline \multirow{2}{*}{ PubMed } & $\begin{array}{l}\text { NADPH } \\
\text { oxidase 1/2 }\end{array}$ & $\begin{array}{l}\text { Nox [tiab] OR NOX } 1 \text { [tiab] OR Nox1 [tiab] OR Nox-1 [tiab] OR NOX } 2 \text { [tiab] OR Nox2 [tiab] OR Nox-2 [tiab] } \\
\text { OR NADPH Oxidase [tiab] OR NADPH oxidases [tiab] OR NADPH-oxidase [tiab] OR NADPH-oxidases } \\
\text { [tiab] OR NAD(P)H oxidase [tiab] OR NAD(P)H oxidases [tiab] OR NAD(P)H-oxidase [tiab] OR NAD(P)H- } \\
\text { oxidases [tiab] OR NOX1/NADPH [tiab] OR NOX2/NADPH [tiab] OR OR NADPH oxidase-derived } \\
\text { superoxide [tiab] OR NADPH oxidase-deficient mice [tiab] glycoprotein gp 91 phox[tiab] OR glycoprotein } \\
\text { gp91 phox[tiab] OR glycoprotein gp91phox[tiab] OR gp 91 phox[tiab] OR gp91phox[tiab] OR Gp91phox } \\
\text { [tiab] OR gp91-phox [tiab] OR gp91(phox) [tiab] OR NOX1/NADPH [tiab] OR "NADPH Oxidase"[Mesh] OR } \\
\text { "NADH, NADPH Oxidoreductases"[Mesh] OR "NADPH oxidase 1" [Supplementary Concept] }\end{array}$ \\
\hline & Animal & Search filter for animal studies Pubmed ${ }^{1}$ \\
\hline
\end{tabular}


Table S1, continued

\begin{tabular}{|c|c|c|}
\hline \multirow[t]{3}{*}{ EMBASE } & $\begin{array}{l}\text { Experimental } \\
\text { stroke }\end{array}$ & $\begin{array}{l}\text { ((brain.ti,ab. OR brains.ti,ab. OR Cerebrovascular.ti,ab. OR cerebral.ti,ab. OR cerebellum.ti,ab. OR } \\
\text { cortical.ti,ab. OR intracranial.ti,ab. OR intracerebral.ti,ab. OR infratentorial.ti,ab. OR supratentorial.ti,ab. OR } \\
\text { hemisphere.ti,ab. OR hemispheric.ti,ab. OR MCA.ti,ab. OR ACA.ti,ab. OR anterior circulation.ti,ab. OR } \\
\text { posterior circulation.ti,ab. OR neuron.ti,ab. OR neuronal.ti,ab.) AND (ischemia.ti,ab. OR ischaemia.ti,ab. OR } \\
\text { infarct.ti,ab. OR infarcts.ti,ab. OR infarction.ti,ab. OR infarctions.ti,ab. OR occlusion.ti,ab. OR } \\
\text { occlusions.ti,ab. OR obstruction.ti,ab. OR obstructions.ti,ab. OR stenose.ti,ab. OR stenoses.ti,ab.)) OR } \\
\text { ((lacunar.ti,ab. OR cortical.ti,ab.) AND (infarct.ti,ab. OR infarcts.ti,ab. OR infarction.ti,ab. OR } \\
\text { infarctions.ti,ab.)) OR ((ACA.ti,ab. OR MCA.ti,ab. OR middle cerebral artery.ti,ab. OR anterior cerebral } \\
\text { artery.ti,ab. OR Posterior Cerebral Artery.ti,ab.) AND ( infarct.ti,ab. OR infarcts.ti,ab. OR infarction.ti,ab. OR } \\
\text { infarctions.ti,ab. OR occlusion.ti,ab. OR occlusions.ti,ab. OR obstruction.ti,ab. OR obstructions.ti,ab.)) OR } \\
\text { Stroke.ti,ab. OR Strokes.ti,ab. OR Vascular Accident.ti,ab. OR Cerebrovascular Accident.ti,ab. OR } \\
\text { Cerebrovascular Accidents.ti,ab. OR CVA.ti,ab. OR Venous Infarction.ti,ab. OR Anterior Cerebral Circulation } \\
\text { Infarction.ti,ab. OR MCAO.ti,ab. OR Apoplexy.ti,ab. OR hemisphericstroke.ti,ab. OR exp brain ischemia/ OR } \\
\text { exp stroke/ OR exp brain infarction/ OR exp brain hemorrhage/ OR "I/R".ti,ab. OR "IRl".ti,ab. OR "Ischemic } \\
\text { reperfusion injury".ti,ab. OR "Ischaemic reperfusion injury".ti,ab. OR "Ischemic reperfusion injuries".ti,ab. OR } \\
\text { "cerebral Ischaemic reperfusion ".ti,ab. OR "cerebral Ischemic reperfusion".ti,ab. OR "reperfusion } \\
\text { injury".ti,ab. OR "reperfusion injuries".ti,ab. OR "ischemia reperfusion".ti,ab.OR "ischaemia reperfusion".ti,ab. }\end{array}$ \\
\hline & $\begin{array}{l}\text { NADPH } \\
\text { oxidase } 1 / 2\end{array}$ & $\begin{array}{l}\text { Nox.ti,ab. or NOX 1.ti,ab. or Nox1.ti,ab. or Nox-1.ti,ab. or NOX 2.ti,ab. or Nox2.ti,ab. or Nox-2.ti,ab. or } \\
\text { NADPH Oxidase.ti,ab. or NADPH oxidases.ti,ab. or NADPH-oxidase.ti,ab. or NADPH-oxidases.ti,ab. or NAD } \\
\text { P H oxidase.ti,ab. or NAD P H oxidases.ti,ab. or NOX1 NADPH.ti,ab. or NOX2 NADPH.ti,ab. or NADPH } \\
\text { oxidase-derived superoxide.ti,ab. or NADPH oxidase-deficient mice.ti,ab. or Gp91phox.ti,ab. or glycoprotein } \\
\text { gp } 91 \text { phox.ti,ab. or glycoprotein gp91 phox.ti,ab. or glycoprotein gp91phox.ti,ab. or gp } 91 \text { phox.ti,ab. or } \\
\text { gp91phox.ti,ab. or gp91-phox.ti,ab. or gp91 phox.ti,ab. or exp reduced nicotinamide adenine dinucleotide } \\
\text { phosphate oxidase/ or exp reduced nicotinamide adenine dinucleotide phosphate oxidase } 1 / \text { or exp reduced } \\
\text { nicotinamide adenine dinucleotide phosphate oxidase } 2\end{array}$ \\
\hline & $\begin{array}{l}\text { Component 3: } \\
\text { Animal }\end{array}$ & Search filter for animal studies in Embase ${ }^{2}$ \\
\hline
\end{tabular}


Table S2. Characteristics of the studies included in the meta-analysis.

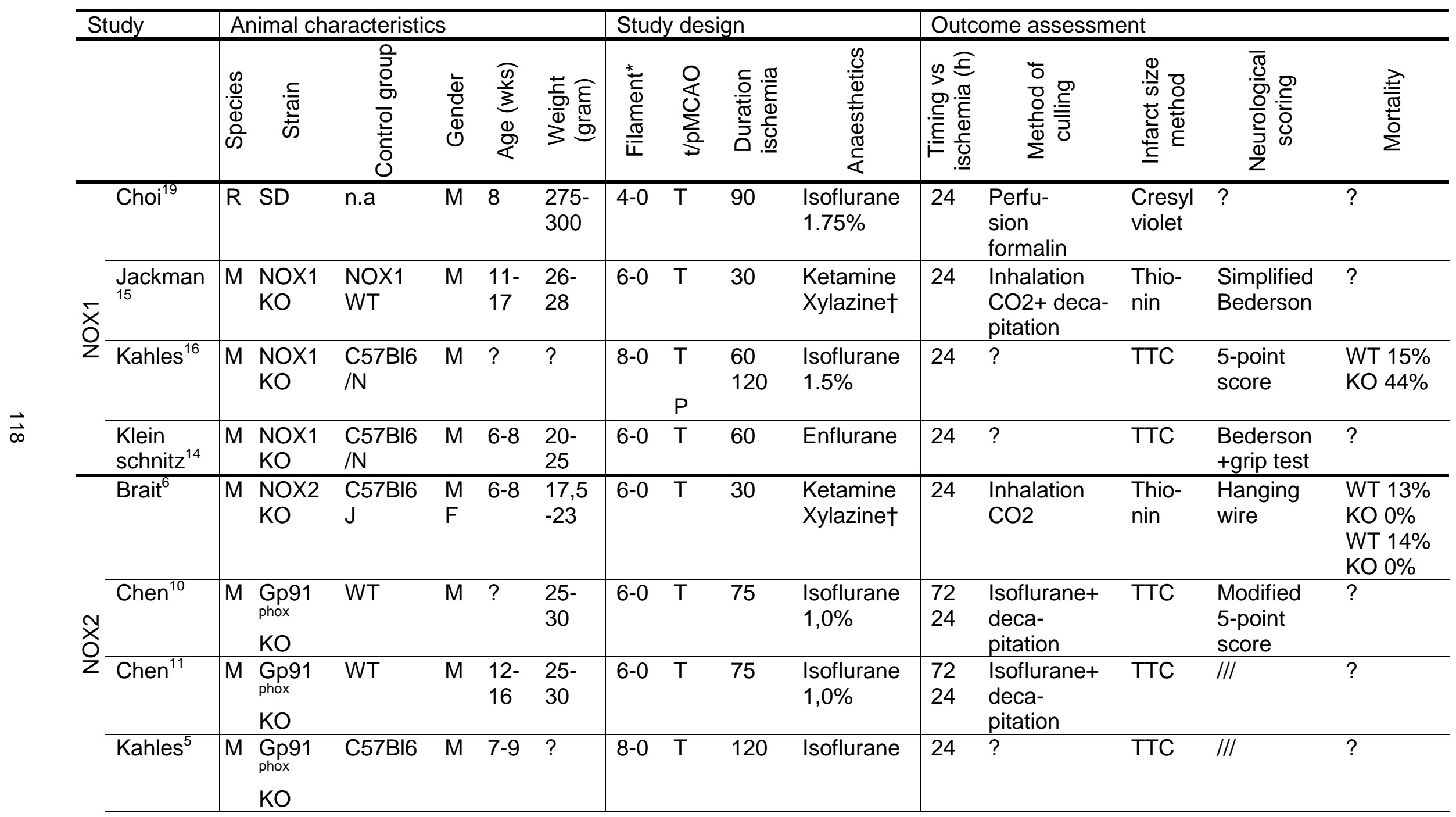


Table S2, continued

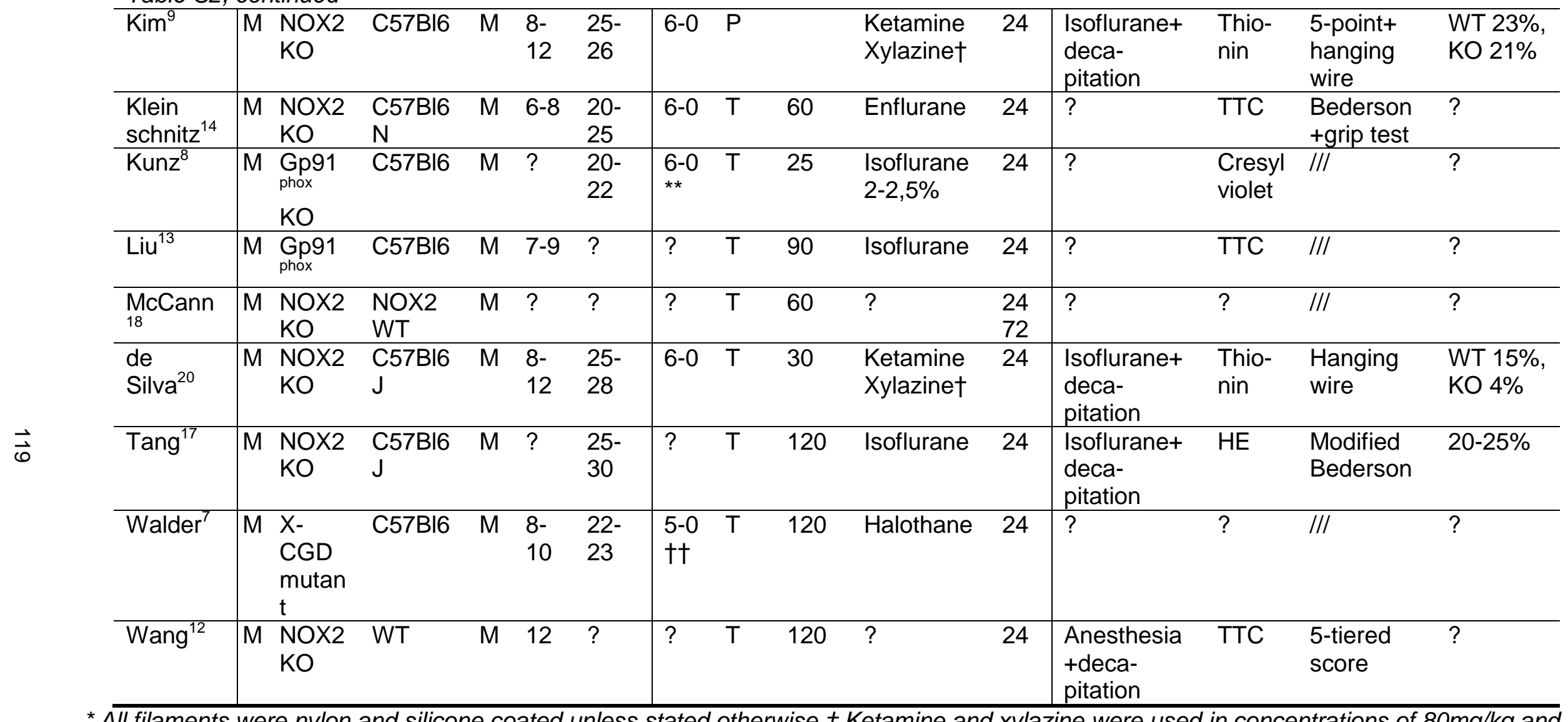
$10 \mathrm{mg} / \mathrm{kg}$ respectively in all studies ** heat blunted $+\dagger$ tip flamed. 
Table S3. Items used for risk of bias assessment.

\begin{tabular}{|c|c|c|}
\hline $\begin{array}{l}\text { Item } \\
\text { no }\end{array}$ & Item description & $\begin{array}{l}\text { Sort of bias } \\
\text { assessed }\end{array}$ \\
\hline 1 & $\begin{array}{l}\text { Was it reported that the experiments were randomised at any } \\
\text { level? }\end{array}$ & $\begin{array}{l}\text { Selection bias } \\
\text { (reporting) }\end{array}$ \\
\hline 2 & $\begin{array}{l}\text { Were the groups similar at baseline or was adjusted for } \\
\text { confounders in the analysis? }\end{array}$ & Selection bias \\
\hline 3 & Was it reported that the study was blinded at any level? & $\begin{array}{l}\text { Selection bias } \\
\text { (reporting) }\end{array}$ \\
\hline 4 & Were the animals randomly housed during the experiment? & Selection bias \\
\hline 5 & $\begin{array}{l}\text { Were the investigators during the course of the experiment } \\
\text { adequately blinded from knowledge of which intervention each } \\
\text { animal received? }\end{array}$ & Peformance bias \\
\hline 6 & Were animals selected at random for the outcome assessment? & Detection bias \\
\hline 7 & Were incomplete study data adequately addressed?* & Detection bias \\
\hline 8 & $\begin{array}{l}\text { Was the study apparently free of other problems that could } \\
\text { pose a high risk of bias?† }\end{array}$ & Attrition bias \\
\hline
\end{tabular}

* Adequate if: all animals included or reasons for missing unlikely related to outcome, missing numbers and reasons equal across groups, imputed using appropriate methods $\dagger$ Other problems that were taken into account as being a high risk of bias: inappropriate influence of funders, inappropriate statistics, design-specific risk of bias, replacement of dropouts, contamination (pooling of drugs), temperature not kept constant during surgery.

\section{SI Methods}

\section{Assessment of methodological quality and risk of bias}

Risk of bias of the included studies was assessed by two independent reviewers (PWMK and $\mathrm{CH}$ ) using the criteria/items described in Table S3. Most criteria were extracted from SYRCLEs risk of bias tool ${ }^{3}$. To overcome the problem of judging too many items as "unclear risk of bias" because reporting of experimental details on animals, methods and materials is very poor ${ }^{4}$ we added 2 items on reporting (item 1 and item 3).

The possible presence of selection bias (items 1, 2 and 4) detection bias (items 6, 7 and 8 ) performance bias (item 5) and attrition bias (items 9) were judged. 
The score 'yes' indicates low risk of bias, the score 'no' indicates high risk of bias and '?' indicates unknown risk of bias.

\section{Meta-analysis}

To clarify if the discrepancies in literature can be explained by experimental quality or publication bias, we first performed a systematic review and meta-analysis of the effects of NOX1 and NOX2 on infarct size and neurological damage in stroke. Our search strategy described in Table S1 identified 562 records in PubMed and 812 records in EMBASE. After removal of duplicates, a total of 1089 records were screened a first time based on title and abstract, excluding non-in-vivo papers, papers not using ischemic stroke and papers using an unspecific inhibitor of NOX or combining an inhibitor with other therapies. Twenty-five articles were included for full text screening, of which 22 addressed NOX2 and 4 articles addressed NOX1. After full text assessment, 13 papers for the NOX2 study (using in total 162 WT and 171 KO mice) and 4 papers for the NOX1 study (using in total 69 WT and 141 KO mice and 16 rats (8 with and 8 without siRNA)) were included (SI Appendix Fig. S1) for qualitative and quantitative analyses ${ }^{5-19}$.

The characteristics of all studies are summarized in SI Appendix Table S2. All studies, except 1 used NOX KO mice as experimental animals, with 1 study using both genders, the rest only males. One study used rats treated with siRNA. All studies used the middle cerebral artery (MCA) occlusion model, 16 studies occluded transiently allowing reperfusion afterwards, and 2 studies occluded the MCA permanently (1 study used both permanent and transient ischemia). The duration of the ischemia and the reperfusion varied greatly among studies (5 to 120 minutes). Three different methods of infarct size measurement and three different scoring systems for the neurological assessment were used. All retrieved data sets could be taken into account for the meta-analysis regarding infarct size. Neurological scoring was measured in 7 out of 13 NOX2 papers and 3 out of 4 NOX1 studies.

Regarding calculated overall effect sizes, mean differences were not possible because of the heterogeneity in study design (i.e species) and the variety of scales used to determine the outcomes. 
CAMARADES consortium (http://www.dcn.ed.ac.uk/camarades//default.htm) suggest the use of normalised mean difference (NMD), which requires correcting for sham values. However, of all 13 included NOX2 papers, only three reported the use of shams, but not for all groups and all outcome parameters ${ }^{6,12,20}$. None of the others mention any sham animals. Therefore, we applied the standardized mean difference (SMD) which is also regularly used in clinical meta-analyses ${ }^{21}$ A SMD expresses the difference between the groups relative to the standard deviation.

\section{Stroke surgery (tMCAO model)}

The model has previously been established by using cerebral blood flow monitoring above the territory of the middle cerebral artery (MCA) in order to visualize a drop of $70-80 \%$ in blood flow after successful vessel occlusion by Laser Doppler (Moor Instruments Ltd., Devon, UK). After administration of a painkiller (buprenorphine s.c. $0,1 \mathrm{mg} / \mathrm{kg}$, repeated every 12 hours), animals were anesthetized with isoflurane (induction $5 \%$ in air, maintenance $1.5-2.5 \%$ in air). Anaesthesia was maintained by spontaneous ventilation of isoflurane. The animal was placed on a heating-pad (UNO Roestvaststaal BV, Zevenaar, NL) and rectal temperature was maintained at $37.0^{\circ} \mathrm{C}$ using a feedback-controlled infrared light. Focal cerebral ischemia was induced using an intraluminal filament technique. Using a surgical microscope (Wild M5A, Wild Heerbrugg, Gais, $\mathrm{CH}$ ), a midline neck incision was made and the right common and external carotid arteries were isolated and permanently ligated. A microvascular clip was temporarily placed on the internal carotid artery. A silicon-coated nylon monofilament (size 6-0, Doccol Corporation, Redlands, CA, USA) was inserted through a small incision into the common carotid artery and advanced into the internal carotid artery until a resistance is felt. The tip of the monofilament should be located intracranially at the origin of the right middle cerebral artery and thereby interrupting blood flow. The filament was held in place by a tourniquet suture that has been prepared before to prevent dislocation during the ischemic period and the wound was closed. Reperfusion was initiated 1 hour after occlusion by monofilament removal. After the surgery, wounds were carefully sutured and animals were allowed to recover from surgery in a temperature-controlled cupboard. No animal dropouts occurred during surgery. 
In total, we conducted surgery on $58 \mathrm{NOX} 2 \mathrm{KO}$ and $48 \mathrm{C} 57 \mathrm{Bl} / 6$ control animals at two study sites. The success rate of the tMCAO procedure was high, with only one NOX2 KO and 5 WT animal producing no neurological or histological signs of brain damage after the surgery, which were subsequently excluded from further analysis. Acute mortality was low ( $10 \%$ in NOX2 KO, $\mathrm{n}=6 ; 4 \%$ in $\mathrm{WT}, \mathrm{n}=2)$ and intrinsic to the stroke pathology (as verified by autopsy).

\section{Neurological behavior}

The mice were assessed for neurological behaviour just before sacrifice to determine the final functional status of the animal. Neurological deficits of the mice that had undergone stroke surgery were measured in a blinded manner on a 0 to 5 scale by using the Bederson Score ${ }^{21}$ with the following definitions: Score 0, no apparent neurological deficits; 1 , body torsion and forelimb flexion; 2 , right side weakness and thus decreased resistance to lateral push; 3, unidirectional circling behavior; 4, longitudinal spinning; 5, no movement.

\section{Motor function}

Prior to sacrifice, the mice were also scored for neurological motor deficit according to the Grip Test ${ }^{22}$. Each mouse was given a discrete value from 0 to 5 . This score is used to evaluate motor function and coordination. The apparatus is a metallic rod (0.22 cm diameters, $50 \mathrm{~cm}$ length) between two vertical supports at a height of $40 \mathrm{~cm}$ over a flat surface. The animal is placed mid-way on this rod and is rated according to the following system: Score 0, falls off; 1, hangs on to string by one or both fore paws; 2 , as for 1 , and attempts to climb on to string; 3 , hangs on to string by one or both fore paws plus one or both hind paws; 4 , hangs on to string by fore and hind paws plus tail wrapped around string; 5, escape (towards the supports).

\section{Infarct Volume Measurements}

The ischemic lesion was measured 24 hours after MCAO. The mice were sacrificed by decapitation. The brain was carefully dissected out, freed from the dura mater and placed in a coronal mouse brain slicer (Zivic Instruments, Pittsburgh, PA, USA). 
Three sequential, $2 \mathrm{~mm}$-thick coronary slices of the brain were cut with razor blades directed by the brain matrix. The slices were then soaked for $10 \mathrm{~min}$ in a freshlyprepared solution of $2 \%$ 2,3,5- triphenyl tetrazolium hydrochloride (TTC, SigmaAldrich, Zwijndrecht, NL) in PBS ( $\mathrm{pH} \mathrm{7.4)} \mathrm{in} \mathrm{a} \mathrm{small} \mathrm{Petri} \mathrm{dish,} \mathrm{maintained} \mathrm{at} 37^{\circ} \mathrm{C}$ in a heater. Excess TTC was then drained, and the slices were washed with PBS and then photographed under the microscope (Dino-Lite Microscope Eyepiece Camera AM 423X). Each brain section was photographed with a ruler. The digital photographs of the TTC stained brain sections were imported into an image analysis program using the software "Leica QWin Pro" for infarct volume measurement. The program quantitatively measures (in $\mathrm{mm} 2$ ) the areas of the infarcted region, the areas of the left and right hemisphere and the total area of the brain slice. Total infarct volume was calculated by adding up infarct volumes of the different sections: $V_{\text {infarct }}$ section $1\left(\mathrm{~mm}^{3}\right)=$ infarct area section $1\left(\mathrm{~mm}^{2}\right) \times$ slice thickness $(2 \mathrm{~mm})$. Infarct volumes are corrected for brain edema according to the following equation: $V_{\text {corrected }}\left(m^{3}\right)=V_{\text {infarct }} \times\left(1-\left(V_{i}-V_{c}\right) / V_{c}\right)$ with $V_{i}-V_{c}$ representing the volume difference between the ipsilateral $\left(V_{i}\right)$ and contralateral $\left(V_{c}\right)$ hemisphere and $\left(V_{i}-V_{c}\right) / V_{c}$ expressing this difference as $\%$ of the control hemisphere. Brain edema volume was calculated by subtracting corrected from uncorrected infarct volumes ${ }^{22}$.

\section{SI References}

1. Hooijmans, C. R., Tillema, A., Leenaars, M. \& Ritskes-Hoitinga, M. Enhancing search efficiency by means of a search filter for finding all studies on animal experimentation in PubMed. Lab. Anim. 44, 170-175 (2010).

2. de Vries, R. B. M., Hooijmans, C. R., Tillema, A., Leenaars, M. \& RitskesHoitinga, M. A search filter for increasing the retrieval of animal studies in Embase. Lab. Anim. 45, 268-270 (2011).

3. Hooijmans, C. R. et al. SYRCLE's risk of bias tool for animal studies. BMC Med Res Methodol 14, 43 (2014).

4. Kilkenny, C. et al. Survey of the quality of experimental design, statistical analysis and reporting of research using animals. PLOS ONE 4, e7824 (2009).

5. Kahles, T. et al. NADPH oxidase plays a central role in blood-brain barrier damage in experimental stroke. Stroke 38, 3000-3006 (2007). 
6. Brait, V. H. et al. Mechanisms contributing to cerebral infarct size after stroke: gender, reperfusion, T lymphocytes, and Nox2-derived superoxide. J Cereb Blood Flow Metab 30, 1306-1317 (2010).

7. Walder, C. E. et al. Ischemic stroke injury is reduced in mice lacking a functional NADPH oxidase. Stroke 28, 2252-2258 (1997).

8. Kunz, A., Anrather, J., Zhou, P., Orio, M. \& Iadecola, C. Cyclooxygenase-2 does not contribute to postischemic production of reactive oxygen species. $J$ Cereb Blood Flow Metab 27, 545-551 (2007).

9. Kim, H. A. et al. Brain infarct volume after permanent focal ischemia is not dependent on Nox2 expression. Brain Res. 1483, 105-111 (2012).

10. Chen, H., Song, Y. S. \& Chan, P. H. Inhibition of NADPH oxidase is neuroprotective after ischemia-reperfusion. J Cereb Blood Flow Metab 29, 1262-1272 (2009).

11. Chen, H., Kim, G. S., Okami, N., Narasimhan, P. \& Chan, P. H. NADPH oxidase is involved in post-ischemic brain inflammation. Neurobiol Dis 42, 341348 (2011).

12. Wang, Z. et al. NOX2 deficiency ameliorates cerebral injury through reduction of complexin II-mediated glutamate excitotoxicity in experimental stroke. Free Radic Biol Med 65, 942-951 (2013).

13. Liu, W., Chen, Q., Liu, J. \& Liu, K. J. Normobaric hyperoxia protects the blood brain barrier through inhibiting Nox2 containing NADPH oxidase in ischemic stroke. Med Gas Res 1, 22 (2011).

14. Kleinschnitz, C. et al. Post-stroke inhibition of induced NADPH oxidase type 4 prevents oxidative stress and neurodegeneration. PLOS Biol. 8, (2010).

15. Jackman, K. A., Miller, A. A., Drummond, G. R. \& Sobey, C. G. Importance of NOX1 for angiotensin II-induced cerebrovascular superoxide production and cortical infarct volume following ischemic stroke. Brain Res. 1286, 215-220 (2009).

16. Kahles, T. et al. NADPH oxidase Nox1 contributes to ischemic injury in experimental stroke in mice. Neurobiol Dis 40, 185-192 (2010).

17. Tang, X. N., Zheng, Z., Giffard, R. G. \& Yenari, M. A. Significance of marrowderived nicotinamide adenine dinucleotide phosphate oxidase in experimental ischemic stroke. Ann. Neurol. 70, 606-615 (2011). 
18. McCann, S., DUSTING, G. \& Roulston, C. 'Time-dependent superoxide generation in NOX2 knockout mice following middle cerebral artery occlusion and reperfusion' in Abstracts of the 6th International Symposium on Neuroprotection and Neurorepair. Rostock, Germany. October 1-4, 2010. (2010).

19. Choi, D.-H. et al. 'Role of NADPH oxidase 1 in ischemia/reperfusion-induced brain injury after stroke' in Abstracts of the World Stroke Congress 2010. October 13-16, 2010. Seoul, Republic of Korea. (2010).

20. De Silva, T. M., Brait, V. H., Drummond, G. R., Sobey, C. G. \& Miller, A. A. Nox2 oxidase activity accounts for the oxidative stress and vasomotor dysfunction in mouse cerebral arteries following ischemic stroke. PLOS ONE 6, e28393 (2011).

21. The Cochrane Collaboration. Cochrane Handbook for systematic reviews of interventions. at <www.cochrane-handbook.org>

22. Kraft, P. et al. Deficiency of vasodilator-stimulated phosphoprotein (VASP) increases blood-brain-barrier damage and edema formation after ischemic stroke in mice. PLoS ONE 5, e15106 (2010). 


\section{Chapter 5}

\section{To the toe: organ specificity and different isoforms}

To determine if NOX4 plays a role in ischemia-reperfusion injury in other peripheral organs and to elucidate possible additional roles of other isoforms, NOX1, NOX2, and NOX5, KO and KI mice were subjected to heart ischemia(-reperfusion) and chronic ischemia of the hindlimb. 
Chapter 5, Part 1

Mouse matter(s) 


\section{Introduction}

Genetically modified mice are a very commonly used tool in biomedical research. Breeding and maintenance of the mice is not often described in published animal studies and also not generally taken into account as a possible explanation for diverging results with in vivo research. We here show that the maintenance of genetically modified mouse lines can definitely influence the results when doing preclinical in vivo studies.

To explore if new and selective NOX inhibitors are effective in the treatment of stroke induced brain damage (the new drugs are not part of this thesis), we subjected NOX4 WT and KO mice to TMCAO and treated them with either the inhibitors or vehicle. WT mice receiving the inhibitors did not show a different infarct size or neurological scoring than the WT mice treated with vehicle (data not shown). Additionally, we had a very surprising finding: we could not reproduce the protective findings of the NOX4 $\mathrm{KO}$ as we published in $2010^{1}$. The infarct sizes of the NOX4 KO treated with vehicle $\left(79,4 \pm 8,4 \mathrm{~mm}^{3}, \mathrm{n}=15\right)$ were comparable to the WT treated with vehicle $\left(83,4 \pm 7,6 \mathrm{~mm}^{3}\right.$, $\mathrm{n}=14$ ), (Fig. $1 \mathrm{~A}$ and $\mathrm{B}$ ). Also, the neurological scores did not differ between our NOX4 KO and WT mice (Fig. 1C and D). Post-study genotyping confirmed that the NOX4 gene was still absent in the NOX4 KO's, excluding a genetic mix up of animals.

\section{Mouse colony time line}

Trying to explain this discrepancy, we analysed the timeline of our NOX4 KO colony from its generation onwards (Fig. 1). The generation of the mouse line was conducted at Murinus in Giessen in 2002-2003. Screening at the German Mouse Clinic did not reveal any basal phenotype in the NOX4 KO mice. Mice were kept at the animal house at the university of Giessen. At this location, the initial experiments showing protection from stroke in the NOX4 KO mice were performed. When the research group moved to Australia in 2005, the mice were transported to Melbourne and housed there at the institutional animal facility called 'Mouseworks'. In Melbourne, the mouse line was cryopreserved. 
From Giessen, a subgroup of mice was taken to Würzburg to the new lab of C. Kleinschnitz to maintain a new colony (NOX4Wü). In Australia, a few mice were sent to a collaborator at the Baker Heart and Diabetes Institute to also start an independent colony (NOX4Me). The original Mouseworks' colony in Melbourne was cryopreserved and thereafter, both the frozen embryos and the living mouse line were moved to the ARMI (Australian Regenerative Medicine Institute). In 2010, the research group came back to Europe. The mice were housed at the central animal facility (CPV) of Maastricht University (NOX4Ma). In 2012 an embryo transfer of the NOX4Ma mice into Harlan was done. Later in the same year, the mice were transferred from Harlan to the new Dutch animal facility 'BioXpert'. The Maastricht line is currently still bred there. The embryos that were cryopreserved at 'Mouseworks' were also transported to Maastricht University and BioXpert. In all these years of breeding, homozygous breeding of the WT and KO lines was done separately. The NOX4Ma mice are currently in approximately the $40^{\text {th }}$ generation of this homozygous breeding.

\section{Different colonies of the same mouse line have different phenotypes in the tMCAO stroke model}

Repeating the stroke experiments in the NOX4Wü mice at the lab of C. Kleinschnitz (who did the initial experiments in 2010) showed a reduction in infarct size (WT $84,5 \pm 4,3 \mathrm{~mm}^{3}, \mathrm{n}=5$; KO $34,1 \pm 6,2 \mathrm{~mm}^{3}, \mathrm{n}=7$ ) (Fig. $2 \mathrm{~A}$ and $\mathrm{B}$ ), but not as striking as the initial experiments. To exclude a possible role of differently performed surgeries or institute related differences, the NOX4Ma mice were also tested in Würzburg, showing no effect of NOX4 on ischemic stroke (data not shown). In addition, the mouse colony from the Baker Institute (NOX4Me) was tested, also showing an almost fully preserved phenotype in stroke concerning infarct size (WT 84,0 $\pm 8,7 \mathrm{~mm}^{3}$, $\mathrm{n}=8$; $\mathrm{KO} 38,7 \pm 9,2 \mathrm{~mm}^{3}, \mathrm{n}=8$ ) (Fig. $1 \mathrm{~A}$ and $\mathrm{B}$ ). Looking at the neurological scores (Bederson and grip test) in the models, only NOX4 KO mice from the Baker institute were protected (Fig. $1 C$ and $D$ ). These data indicate that during the years, the NOX4 KO Ma mouse colony has lost its stroke phenotype while the NOX4 gene is still absent. 
Interestingly, this seems to be specific for the stroke damage since the NOX4Me mice do show a phenotype in diabetic nephropathy ${ }^{2}$ and the NOX4Ma have a phenotype related to glucose metabolism and are hypertensive when aged (unpublished data).

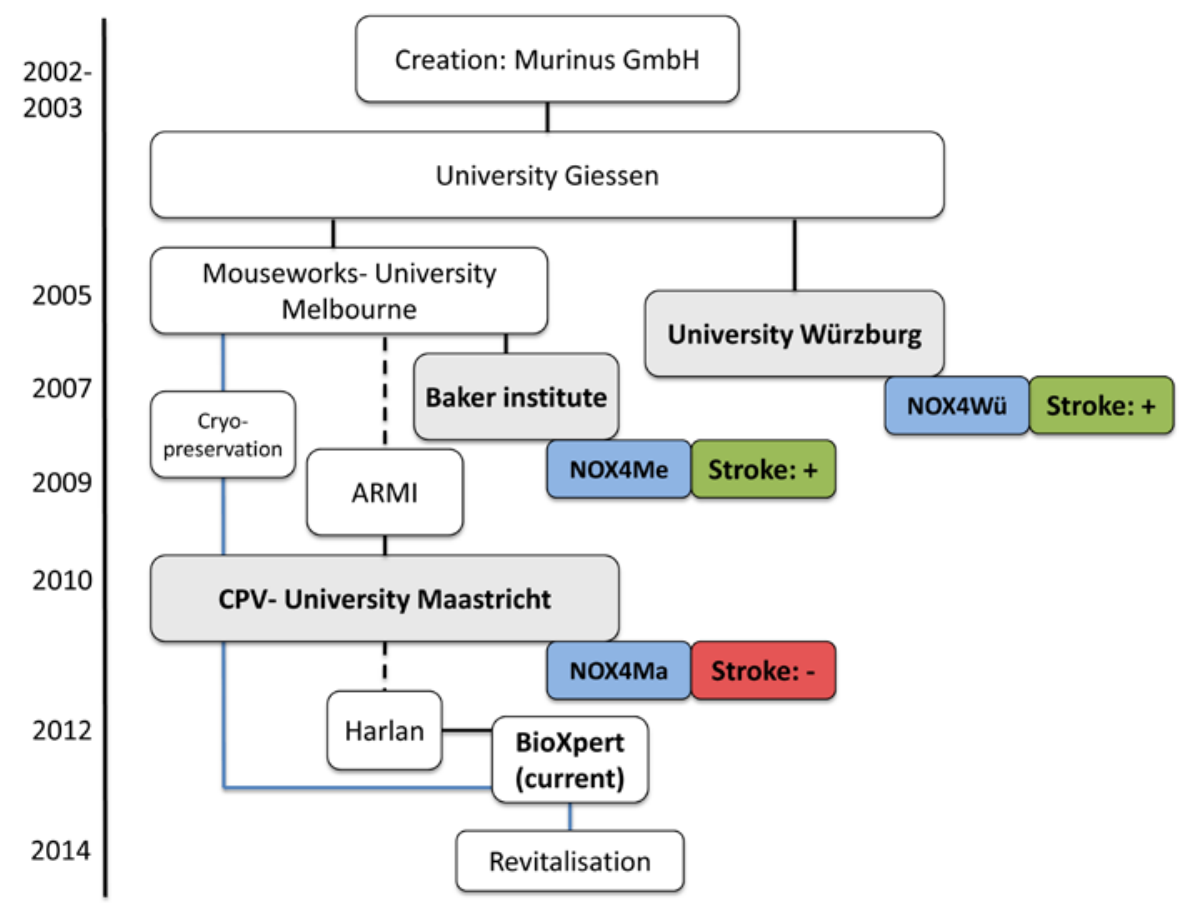

Figure 1. Overview of the NOX4 KO mouse line over time. Solid black lines represent transfers. Dashed black lines represent embryo transfers. The blue solid line depicts the cryopreservation and the shipment of those embryos to Maastricht and BioXpert. In the blue boxes, the 3 different lines that were tested for stroke are named. Green and red boxes depict the presence $(+$, green) or absence $(-$, red) of the stroke phenotype.

\section{Experimental mouse breeding: how to prevent phenotypic losses}

Breeding inbred strains of mice can result in genetic instability over the years. Even several sub strains of the C57Bl6 mice are not so genetically identical as was thought ${ }^{3,4}$. Several reasons for this are discussed by Casellas ${ }^{5}$. Mutations in genes are a naturally occurring event, which cannot be prevented. Single gene mutations but also polygenic mutations can lead to changes in the genetic background. If the genetic variability occurs spontaneously, this is called genetic drift. 
During all the years of keeping the colony the mice were transported from one place to another a few times. This leads to a change in environment to which the mice have to adapt.

A
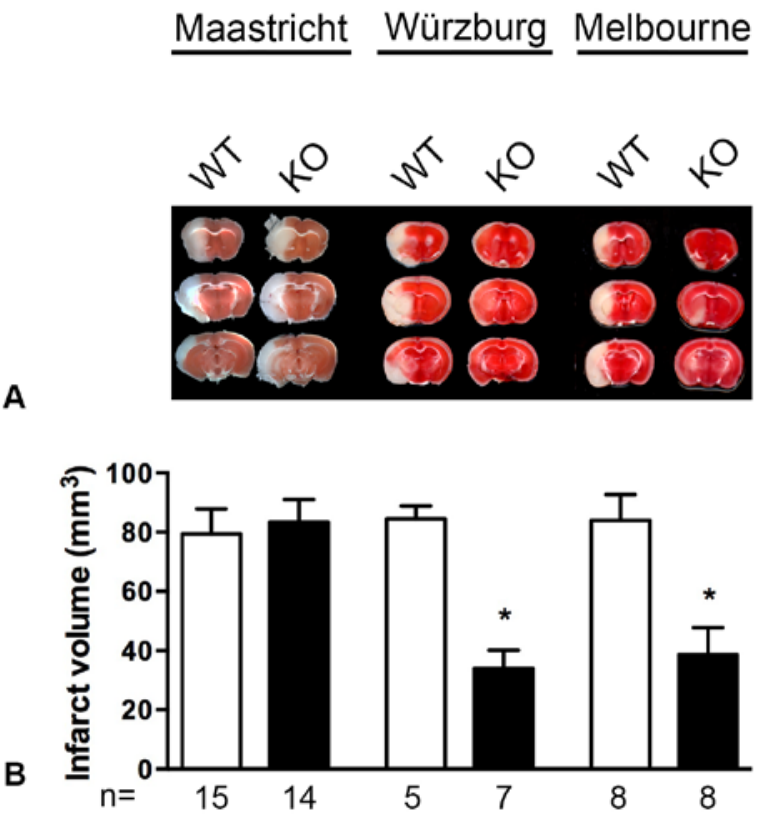

Maastricht Würzburg Melbourne

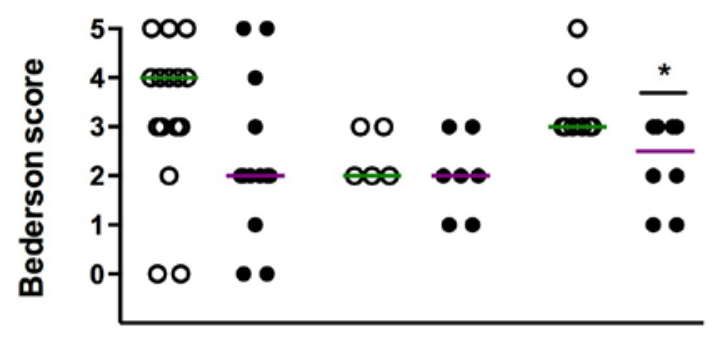

C

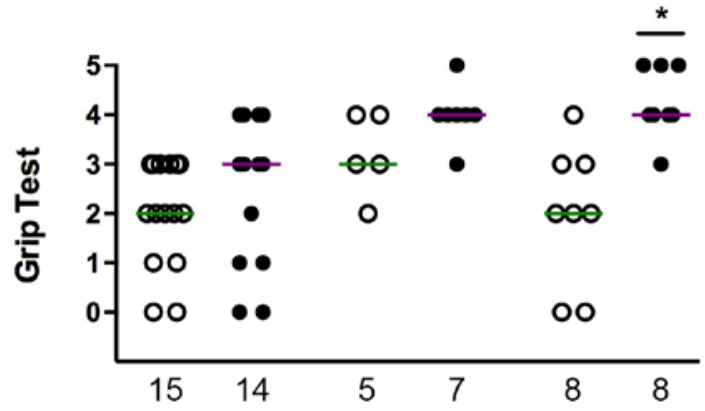

Figure 2. Three different colonies of the NOX4 KO mice show different stroke phenotypes.

A) Representative TTC staining of three sequential coronal brain sections on day 1 after tMCAO. B) Infarct volumes in NOX4 KO (black bars) were decreased compared to the NOX4 WT (white bars) mice in the Wurzburg and Melbourne colonies, but not in the Maastricht colony. Bars represent mean \pm SEM of $n$ experiments as mentioned below each column. C) Bederson scores ( $0=$ normal to $5=$ severe deficit) were only better in the Melbourne NOX4 $K O$ (closed dots, purple lines) versus the WT (open dots, green lines) mice (scatter plot+means) D) Grip test scores, ( $0=$ severe deficit to $5=$ normal) were not changed in any of the NOX4 KO versus their respective WT. N-numbers are mentioned below the columns.

This may lead to a sorting of specific genes within the colony according to the survival of the fittest. When several breeder pairs per generation are used to maintain a colony, it cannot be excluded that one breeder pair can have a higher reproductive fitness and hence will contribute genetically more to the next breeding generation. This is especially true for breeding heterozygous versus homozygous mice, a phenomenon called heterozygyote selection ${ }^{5}$. The homozygous breeding of our line during all the years, without crossing back to a fresh Bl6 mouse, can be a third factor. 
The naturally occurring genetic drift can accumulate mutations within the mouse colony. Although the gene of interest is not directly affected, small mutations in other genes may have accumulated. Mutations that cause for example dwarfism or changes in fur colour will be easily detected and these mice will be excluded from breeding for further generation. However, silent mutations that do not results in an overt phenotype can carry on in a colony for long times and become fixed ${ }^{6}$. In our case, most probably genes involved somehow in the pathway of ischemiareperfusion injury, were affected by the mutations, leading to a loss of phenotype. Another potential problem of homozygous inbreeding is the occurrence of inbred depression: with an increase in generation numbers, the fitness and reproducing potential of the mice will decrease ${ }^{7}$.

Prevention of inbred depression and accumulation of spontaneous mutations can be accomplished by regularly backcrossing the mouse line to the 'fresh' WT line. We now implemented this in the core (maintenance) breedings of all our different mouse lines at BioXpert (Table 1). Cryopreservation of early generations is another option to refresh the mouse line during the years. At BioXpert, we are currently trying to revitalise the embryos that were frozen at Mouseworks in Melbourne to also test these animals for their phenotype in stroke.

The two following parts of Chapter 5, describing experiments in the heart and hindlimb in different NOX KO mice, were initially performed in the NOX4Ma mice. When discovering the loss of stroke phenotype in these mice, we decided to repeat experiments with the NOX4Wü mice to see if the results found in NOX4Ma mice are reproducible. For the hindlimb, the total group of experiments has already been conducted in both the Ma and Wü mice with no difference between these 2 groups. However, for the heart, we are still in the phase of repeating experiments, thus now the data of the NOX4Ma mice are presented only. Since the two colonies show differences in stroke phenotype but not in the hindlimb, we cannot be sure that the NOX4Ma mice show the real NOX4-related phenotype. Thus, the conclusions that we draw for the heart study are to be interpreted with caution and might need to be rethought when the experiments have been repeated with the NOX4Wü or NOX4Me mice. 
Table 1. Core breeding with backcross to the fresh background strain every $4^{\text {th }}$ generation.

\begin{tabular}{|c|c|c|c|c|}
\hline \multirow[t]{2}{*}{ Generation } & \multicolumn{2}{|c|}{$\begin{array}{c}\text { Breeder pairs: GM not on X } \\
\text { chromosome }\end{array}$} & \multicolumn{2}{|c|}{$\begin{array}{c}\text { Breeder pairs: GM on X- } \\
\text { chromosome }\end{array}$} \\
\hline & GM mouse & $\begin{array}{l}\text { Background } \\
\text { mouse }\end{array}$ & GM mouse & Background mouse \\
\hline N1 & Ho 9 x Ho ơ & & Ho $9 \times \mathrm{Ho}^{\top}$ & \\
\hline N2 & Ho $9 \times$ Ho o & & Ho $9 \times \mathrm{Ho}^{\top}$ & \\
\hline N3 & Ho $9 \times$ Ho o & & Ho $9 \times$ Xo o & \\
\hline B & Ho 9 or Ho o & WT $q$ or $0^{*}$ & Ho 9 & WT o \\
\hline NO & Het $q \times$ Het $\delta^{\top}$ & & Het $q \times$ Het $\sigma^{\star}$ & \\
\hline N1 & Ho $\mathrm{O} \times \mathrm{Ho}^{*}$ & & $\mathrm{Ho}$ \% $\times \mathrm{Ho} \mathrm{o}^{\pi}$ & \\
\hline
\end{tabular}

In generations N1-N3, breeder pairs can be bred approximately 3 times before using the litters for the next generation breeding. For the backcross $(B)$ breeding, use 'fresh' mice from the original background strain. GM: genetically modified; Ho: homozygous; Het: heterozygous 우: female; $0^{7}$ : male.

\section{Mouse models used in Chapter 4}

\section{NOX1 KO mice}

NOX1 y/- animals were kindly provided by Karl-Heinz-Krause and bred at our own animal facility. The generation of these mice has been previously described by Gavazzi et al $^{8}$.

\section{NOX2 KO mice}

B6.129S-Cybb ${ }^{\text {tm1din/J }}$ mice were purchased from Jackson Laboratories (stock number 002365) and bred at our own animal facility.

\section{NOX4 KO mice}

The generation of the total body NOX4 KO mice has been described in detail in ${ }^{1}$. Briefly, a mouse missing the NADPH binding pockets, located in exons 14 and 15 of the NOX4 gene was generated using the CrelLox recombination technique and a targeting vector based on the Litmus28 vector. 


\section{NOX5 KI generation}

Since the rodent genome naturally lacks the NADPH oxidase 5 gene, we created a new mouse line expressing the human NOX5 beta under the control of the endothelial cell specific Tie2 promotor. The model was developed using the hypoxanthine phospho-ribosyl-transferase (Hprt) targeted transgenic approach. This locus protects transgenic constructs inserted into this region against gene silencing, positional or methylation effects, and tissue-specific promotors inserted in the Hprt locus conserve their expression patterns. Importantly, Hprt deficient mice are phenotypically normal.

Targeted insertion at the Hprt locus overcomes the unpredictable position effects inherent in transgenic methods relying on random integration. Thus, a respective $\mathrm{KI}$ line allows experiments to be conducted with a single mouse line, in contrast to the 3-5 transgenic lines usually required using classical technology.

The Hprt gene is localised on the $\mathrm{X}$ chromosome. Thus, it is suggested to analyse hemizygous males or homozygous females.

The targeting vector was obtained by subcloning of a transgenic targeting cassette containing the human NOX5 beta gene under control of the Tie2 promotor into the Hprt targeting vector (GenOway), upstream of the human Growth Hormone polyA sequence.

E14 ES cells of 129SV mice were transfected with the linearised targeting vector, selected with HAT (hypoxanthine-aminopterin-thymidine medium) medium and resistant clones screened (Southern blot) for presence of the 3' and 5' homologous recombination events. Selected ES cells were injected into C57B/6/J blastocysts and re-implanted into OF1 (oestrus phase) pseudo-pregnant females, which resulted in chimeric males. Chimeras were mated with WT C57BI/6 females and agouti female offspring was genotyped to confirm the presence of the recombined $X$ chromosome. The heterozygous females were then mated with transmitting male chimeras to generate hemizygous males and homozygous females. All procedures were carried out under SPF conditions by GenOway, which houses all mice at Charles River. The mice were shipped to Harlan and mated for breeding. Maintenance breeding is now done at BioXpert. 


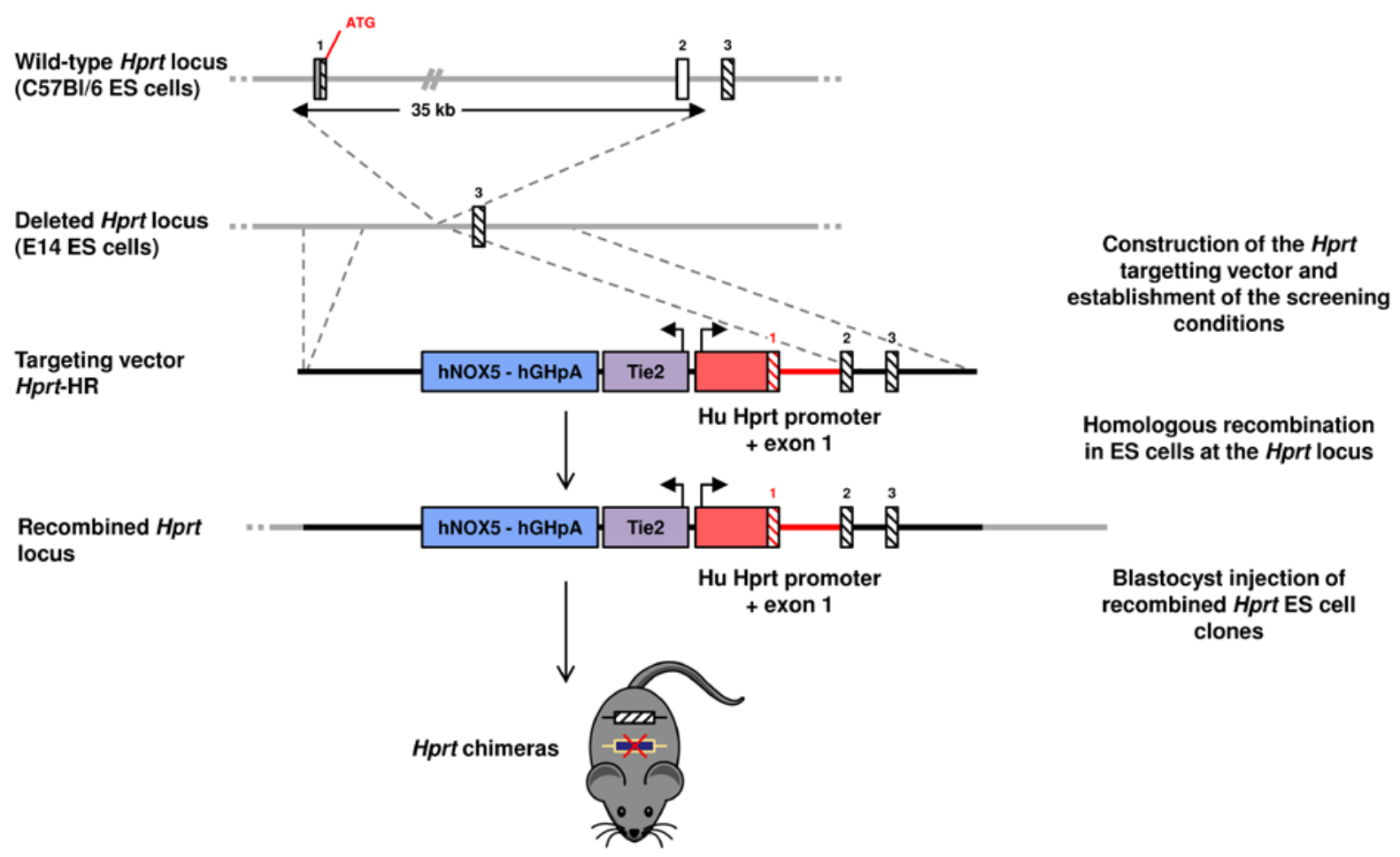

Figure 3. Schematic representation of the construction of human eNO5 KI mice. First, the wild type and deleted Hprt constructs are shown. A targeting vector to insert NOX5 was created: human NOX5 cDNA (blue box) is coupled to the human Hprt promoter (orange box) and exons 1 and 2. The human Hprt promoter is under control of the Tie2 gene (purple box) to create endothelial cell specific expression. In recombined embryonic stem cells, the Hprt gene is then reconstituted by introduction of the targeting vector. The recombined ES cells are then injected into blastocysts to create chimeric mice for further breeding. Hatched grey and red boxes represent murine and human Hprt exons, respectively. Solid line represents intronic sequences. Diagram is not depicted to scale.

ES cells, embryonic stem cells; hGHpA, human growth hormone poly A sequence; Hprt, hypoxanthine phospho-ribosyl-transferase

\section{ACKNOWLEDGMENTS}

We gratefully thank Dr. Elflein for her expert scientific input regarding the NOX4 mouse line loss of phenotype. 


\section{References}

1. Kleinschnitz, C. et al. Post-stroke inhibition of induced NADPH oxidase type 4 prevents oxidative stress and neurodegeneration. PLOS Biol. 8, (2010).

2. Jha, J. C. et al. Genetic targeting or pharmacologic inhibition of NADPH oxidase nox4 provides renoprotection in long-term diabetic nephropathy. J. Am. Soc. Nephrol. 25, 1237-1254 (2014).

3. Zurita, E. et al. Genetic polymorphisms among C57BL/6 mouse inbred strains. Transgenic Res. 20, 481-489 (2011).

4. Mekada, K. et al. Genetic Differences among C57BL/6 Substrains. Exp. Anim. 58, 141-149 (2009).

5. Casellas, J. Inbred mouse strains and genetic stability: a review. Animal 5, 1-7 (2011).

6. Stevens, J. C., Banks, G. T., Festing, M. F. W. \& Fisher, E. M. C. Quiet mutations in inbred strains of mice. Trends in Molecular Medicine 13, 512-519 (2007).

7. Charlesworth, D. \& Willis, J. H. The genetics of inbreeding depression. Nat. Rev. Genet. 10, 783-796 (2009).

8. Gavazzi, G. et al. Decreased blood pressure in NOX1-deficient mice. FEBS Lett. 580, 497-504 (2006). 


\section{Chapter 5, Part 2}

The heart: NOX4 does not play a major role in myocardial infarction or ischemia-reperfusion 


\begin{abstract}
Ischemic heart disease accounts for almost $50 \%$ of all deaths due to cardiovascular diseases and its incidence is still growing. The underlying pathophysiology of ischemia-reperfusion injury represents a challenge for treatment: reperfusion is necessary for tissue survival, but can also increase the damage by ROS formation. NADPH oxidase 4 is a main contributor to stroke damage. Its role in cardiac ischemia(-reperfusion) has not been tested extensively. Acute studies failed to show a role of NOX4 and studies on potential modulation of cardiac wound healing and function on the long-term are missing. Here we examine the contribution of NOX4 in MI and IR mouse models up to 4 weeks after the induction of injury. In addition, we also used the same models in NOX1 and $2 \mathrm{KO}$ and NOX5 KI mice. Our data show that NOX4 does not play a role in myocardial infarction or ischemia reperfusion injury of the heart after 4 weeks. We found minor effects of NOX2 and no effect of NOX1 in our chronic models. Investigating the last vascular NADPH oxidase, NOX5, showed a better preserved contractility 4 weeks after MI but not after ischemia-reperfusion injury of the heart. Thus, NOX4 seems to have a brain specific role in ischemiareperfusion, while NOX2 and 5 might have minor roles in the heart. This underlines that the roles of NADPH oxidases are isoform and tissue specific.
\end{abstract}




\section{Introduction}

Myocardial infarction and ischemia-reperfusion of the heart are both part of the spectrum of ischemic heart diseases. These heart diseases lead to almost $50 \%$ of all cardiovascular disease related deaths worldwide ${ }^{1}$. Treatments have been optimised in the past decades, leading to more survival but also to more morbidity in terms of the development of and predisposure to heart failure. Thus, better treatments are still needed both to decrease mortality and to prevent the development of heart failure.

With ischemia-reperfusion injury and ROS formation ${ }^{2-4}$ being key players in the heart injury associated with myocardial infarction, NADPH oxidases seem logical targets for the development of new drugs. As was shown previously ${ }^{5}$ and in Chapter 2 of this thesis, NOX4 plays a major role in stroke, but data on it's role in heart ischemia are conflicting. Smaller infarcts were found in general as well as cardiac specific NOX4 KO mice ${ }^{6}$ while no difference in the cardiac phenotype was observed in another study in general NOX4 KO mice ${ }^{7}$. The drawback of both studies is that only acute effects were examined, leaving the clinically more important long term-effects unknown up to now. Studies on the involvement of NOX4 in heart remodelling caused by pressure overload, showed contrasting results also ${ }^{8,9}$. The reasons for these discrepant findings remain unclear. NOX4 has also been shown to be involved in angiogenesis ${ }^{10,11}$, VSMC proliferation ${ }^{12,13}$, and in differentiation of fibroblasts into myofibroblasts ${ }^{14}$, factors that all could contribute to remodelling after a myocardial infarction.

The involvement of NOX1 and 2 in ischemia-reperfusion has also been studied by several groups, showing conflicting results in terms of infarcts size, collagen formation and functionality ${ }^{15-19}$.

In addition, NOX5 might be an important player as it has been found to be upregulated in patients with coronary artery disease ${ }^{20}$ and to be increased in cardiomyocytes after myocardial infarction ${ }^{21}$.

In this chapter, the role of NOX1, NOX2, NOX4 and NOX5 in the chronic response to ischemia reperfusion (IR) or myocardial infarction (MI) of the heart will be explored. 


\section{Results}

NOX $\mathrm{KO} / \mathrm{KI}$ and their respective matched WT's were subjected to either permanent ligation of the left descending coronary artery, or transient (45 min) occlusion of the same artery followed by reperfusion. Mice of 8-12 weeks old of both genders were used. After 4 weeks of follow-up, mice were sacrificed. For the NOX4 studies, only the data from the NOX4Ma group are shown (experiments are currently being repeated with NOX4Wü mice), the results should be interpreted with caution because of the lacking stroke phenotype in these mice. Also, the NOX2 IR experiments are still ongoing, thus these data are missing.

\section{Mortality not different in any of the KO or KI groups compared to WT}

First, we analysed mortality to make sure that the subsequent results were not biased by different dropout. Kaplan Meier survival analysis did not show any differences between the different $\mathrm{KO} / \mathrm{KI}$ lines and their respective WT (Fig. 2). Deaths in the myocardial infarction group were mostly due to cardiac rupture at day 3-5 after surgery or heart failure later in the follow-up. In the ischemiareperfusion group, most deaths occurred during or shortly after surgery, due to the severity of the ischemia in combination with the anaesthesia.

The NOX5 mice show a higher rate of mortality due to rupture, which may be due to the different genetic background: mice on a 129SV background show more inflammation and more cardiac ruptures compared to mice on a Bl6 background ${ }^{22}$. Mortality rates are presented in Table 1.

\section{Infarct size not influenced by NADPH oxidases, collagen content only by NOX2}

AZAN staining of paraffin sections shows smaller infarct sizes in the IR group $(9 \pm 1 \%, n=118)$ compared to the $\mathrm{Ml}(32 \pm 2 \%, n=135)$ group $(p<0.0001)$. None of the $\mathrm{KO} / \mathrm{KI}$ mice however showed a decrease or increase in infarct size compared to their WT in either model (Fig. 2 and Table 2). 


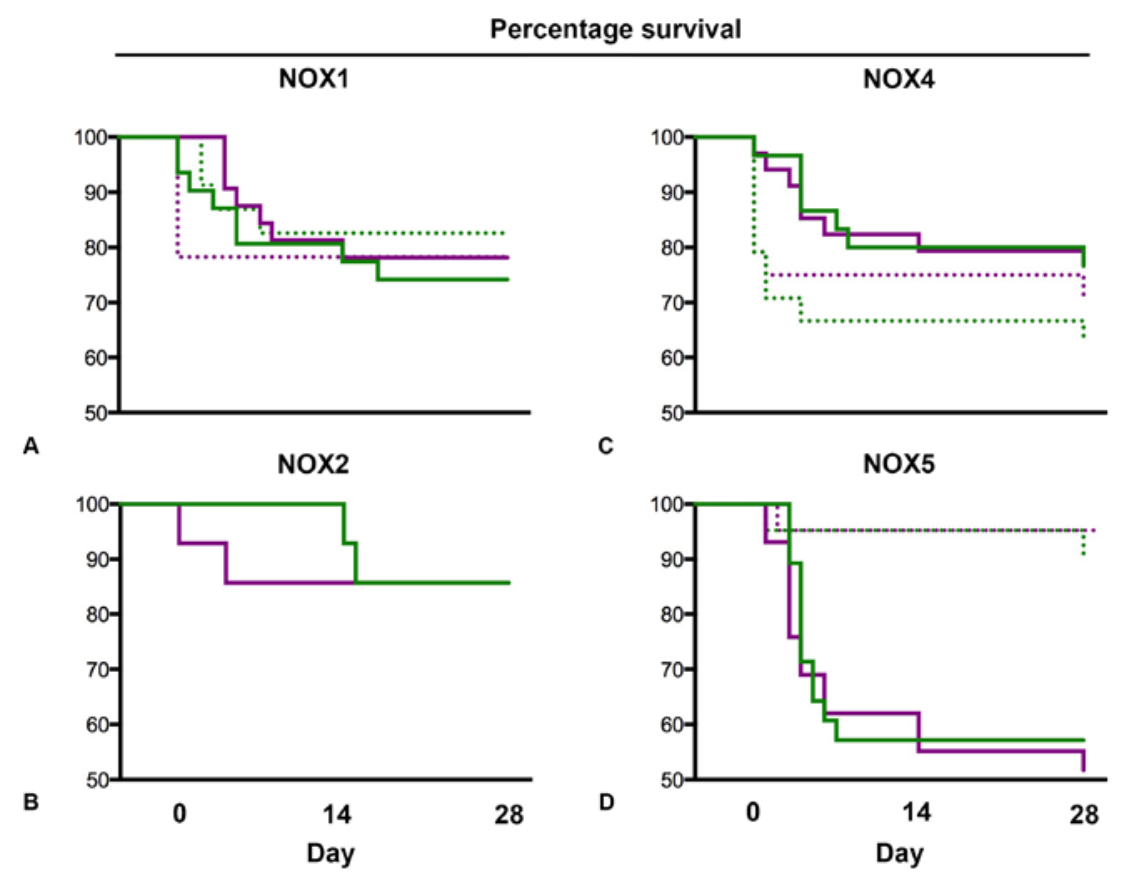

Figure 1. No differences in survival between WT and KO/KI mice after $M I$ or IR of the heart. A) Kaplan Meier Survival curve of NOX1 KO (MI $n=32$, IR $n=23)$ and WT (MI $n=31$, IR $n=23)$ mice. B) Kaplan Meier Survival curve NOX2 KO (MI $n=14)$ and WT MI $(n=14)$. C) Kaplan Meier Survival curve NOX4 KO (MI $n=34$, IR $n=24)$ and WT (MI $n=30$, IR $n=24)$ mice. D) Kaplan Meier Survival curve NOX5 KI $(M I n=29$, IR $n=21)$ and WT (MI $n=28, I R n=21)$ mice. Green lines represent $W T$, purple lines represent $K O / K I$ mice, full lines represent $\mathrm{MI}$, dashed lines represent IR.

Picrosirius red stainings were performed to assess collagen content in the infarct region, border zone and in the septum (Fig. 3 and 4, Table 2). The NOX4 mice did not show any differences in collagen content in any of the three regions. NOX2 KO mice $(47,9 \pm 2,0 \% \mathrm{n}=11)$ showed an increased collagen content only within the infarct zone compared to the NOX2 WT $(37,9 \pm 2,7 \% \mathrm{n}=12)$ after MI. In NOX1 KO and NOX5 KI mice no differences in collagen content were found when compared to the WT mice. 
Table 1. Mortality rates of the different NOX KO/KI and their respective WT groups.

\begin{tabular}{l|rrc|ccc}
\hline & \multicolumn{3}{|c|}{ MI } & \multicolumn{3}{c}{ IR } \\
\hline NOX1 WT & Total & Death & Percentage & Total & Death & Percentage \\
NOX1 KO & 31 & 8 & 26 & 23 & 4 & 17 \\
NOX2 WT & 32 & 7 & 22 & 23 & 5 & 22 \\
NOX2 KO & 14 & 2 & 14 & n.a & & \\
NOX4 WT & 14 & 2 & 14 & & & \\
NOX4 KO & 30 & 7 & 23 & 24 & 9 & 38 \\
NOX5 WT & 34 & 7 & 21 & 24 & 7 & 29 \\
NOX5 KI & 28 & 12 & 43 & 21 & 2 & 10 \\
\hline
\end{tabular}

Hypertrophy was assessed by measuring heart weight divided by body weight. Table 2 shows that in the NOX4 KO mice with a myocardial infarction, cardiac hypertrophy was less pronounced than in their respective WT, but this was not accompanied by any change in infarct size or functionality.
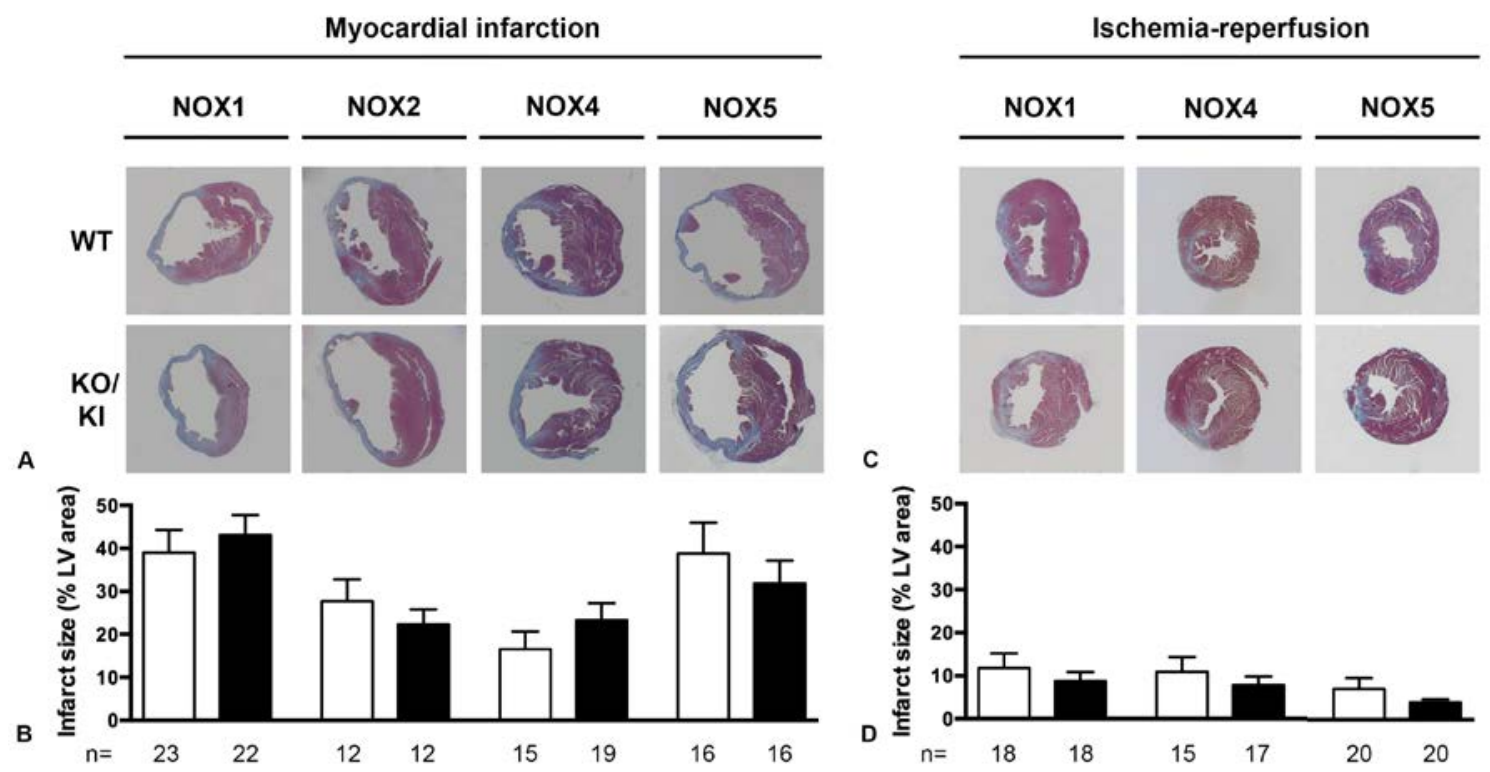

Figure 2. No differences in infarct size 4 weeks after $M I$ or IR of the heart. $A$ and C) Representative pictures of AZAN stain 4 weeks after myocardial infarction (A), respectively ischemia-reperfusion (C) in the different $K O$ and WT groups.

$B$ and $D$ ) Infarct sizes in WT (white bars) and KO/KI (black bars) mice after $M I(B)$ or IR (D) of the heart. Bars express mean \pm SEM of $n$ experiments as indicated below each bar. 


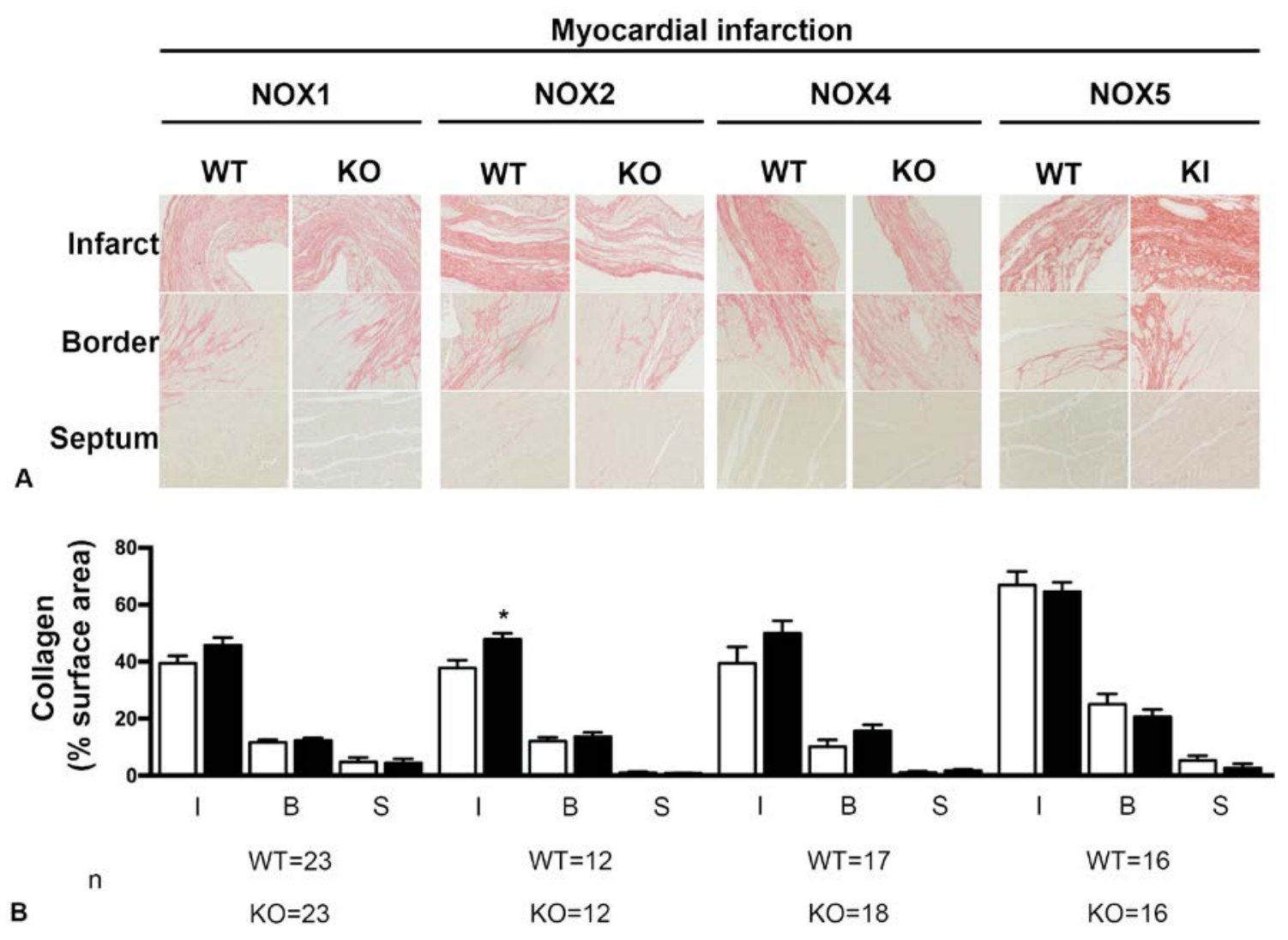

Figure 3. Collagen content in the infarct area (I), border zone (B) and septum (S) 4 weeks after MI. A) Representative pictures of picrosirius red stain in the different $K O$ and WT groups. B) Percentage of collagen content in WT (white bars) and KO/KI (black bars) mice. Bars express mean \pm SEM of $n$ experiments as indicated below each bar. ${ }^{*} p<0,05$ $K O / K I$ vs respective $W T$.

\section{Functionality of the post-MI or post-IR heart affected in NOX2 KO and NOX5} KI mice

To test functionality of the hearts, ultrasounds were performed before surgery and 2 and 4 weeks after MI or IR. Tables 3 and 4 show that all genetically modified mice had baseline values comparable to their matched WT. Mice subjected to myocardial infarction showed a larger functional decline than mice subjected to ischemia-reperfusion. The clinically relevant functional parameter ejection fraction, did not differ in any of the $\mathrm{KO} / \mathrm{KI}$ mice compared to WT mice (Fig. 5). 


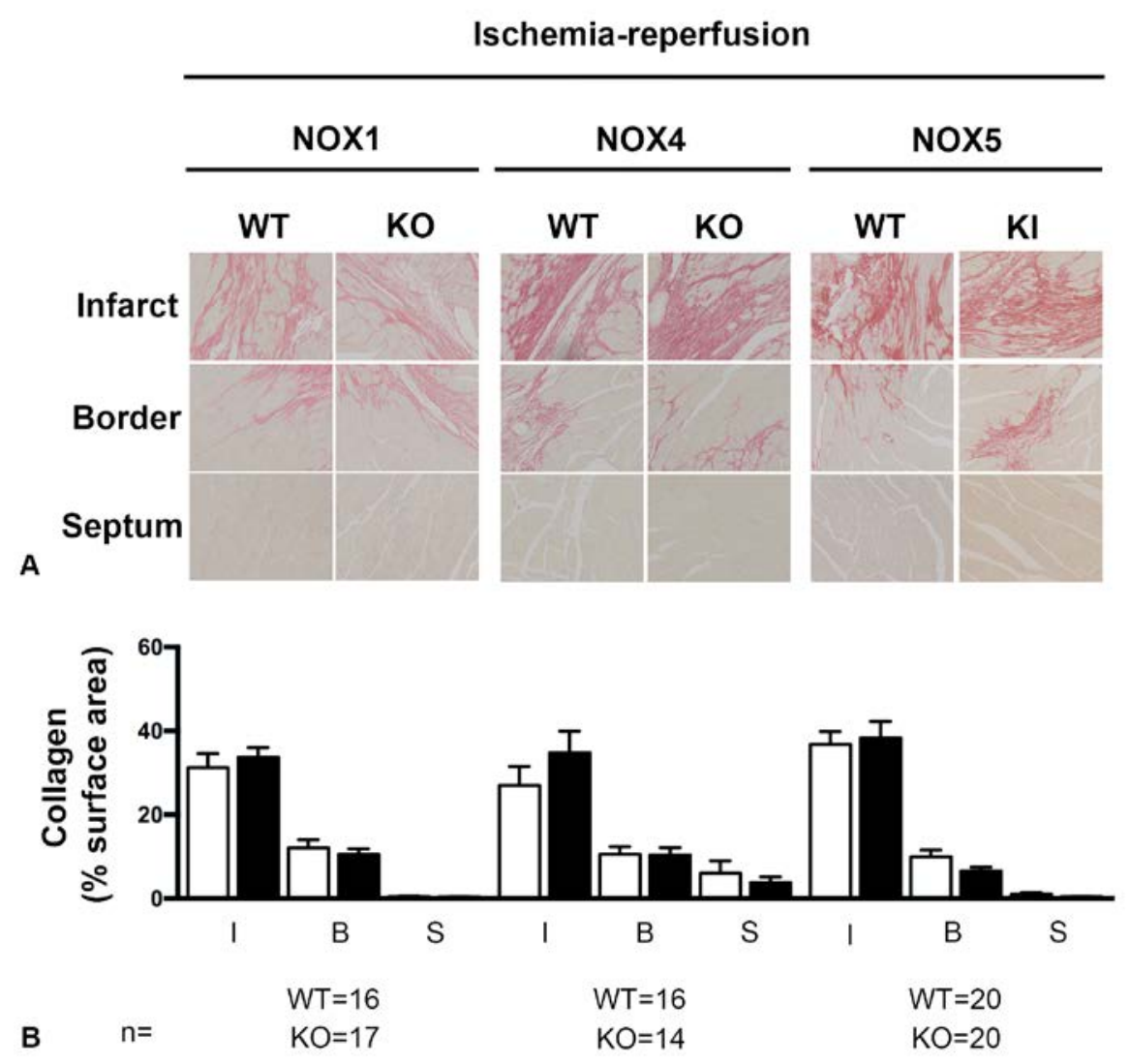

Figure 4. Collagen content in the infarct area (I), border zone (B) and septum (S) 4 weeks after IR. A) Representative pictures of picrosirius red stain in the different $K O$ and WT groups. B) Percentage of collagen content in WT (white bars) and KO/KI (black bars) mice. Bars express mean \pm SEM of $n$ experiments as indicated below each bar ${ }^{*} p<0,05$ $K O / K I$ vs respective $W T$.

Stroke volume and cardiac output also did not differ between any of the genotypes. Dimensional parameters did not show any changes in the structure of the heart in the NOX1/2/4 KO or NOX5 KI mice (Table 3). Thinning of the infarct wall (anterior ventricular wall) was more pronounced in NOX2 KO $(-0,37 \pm 0,04 \mathrm{~mm})$ versus NOX2 WT mice $(0,02 \pm 0,15)$, but no changes in the other NOX groups were found (Table 3 and 4 ). 
Table 2. Overview of the infarct sizes, collagen content and amount of oxidative stress (DHE) in the NOX1, 2 and 4 KO, NOX5 KI and their respective WT mice 4 weeks after myocardial infarction (MI) or ischemia reperfusion (IR).

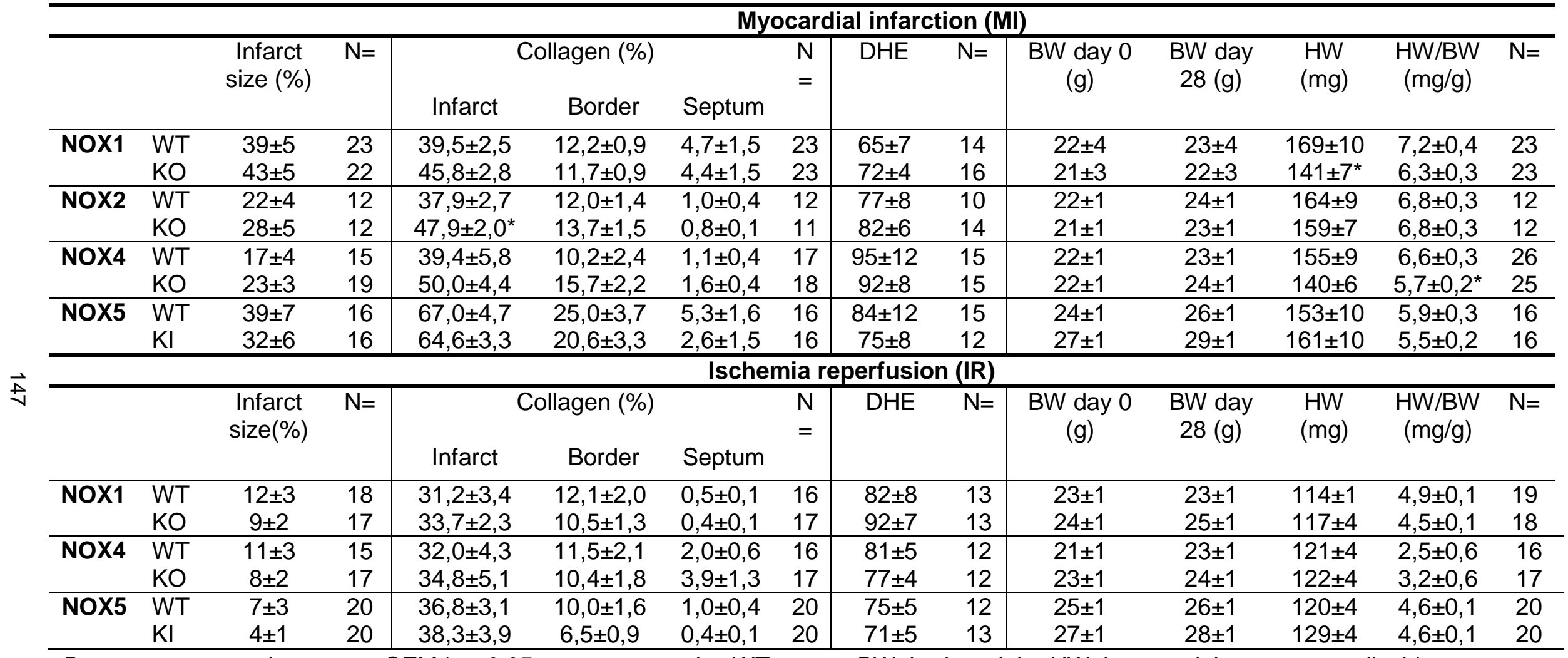

Data are expressed as mean $\pm S E M{ }^{*} p<0.05$ versus respective WT group. BW, body weight; HW, heart weight; n.a not applicable 
Table 3. Ultrasound measurements in the myocardial infarction groups.

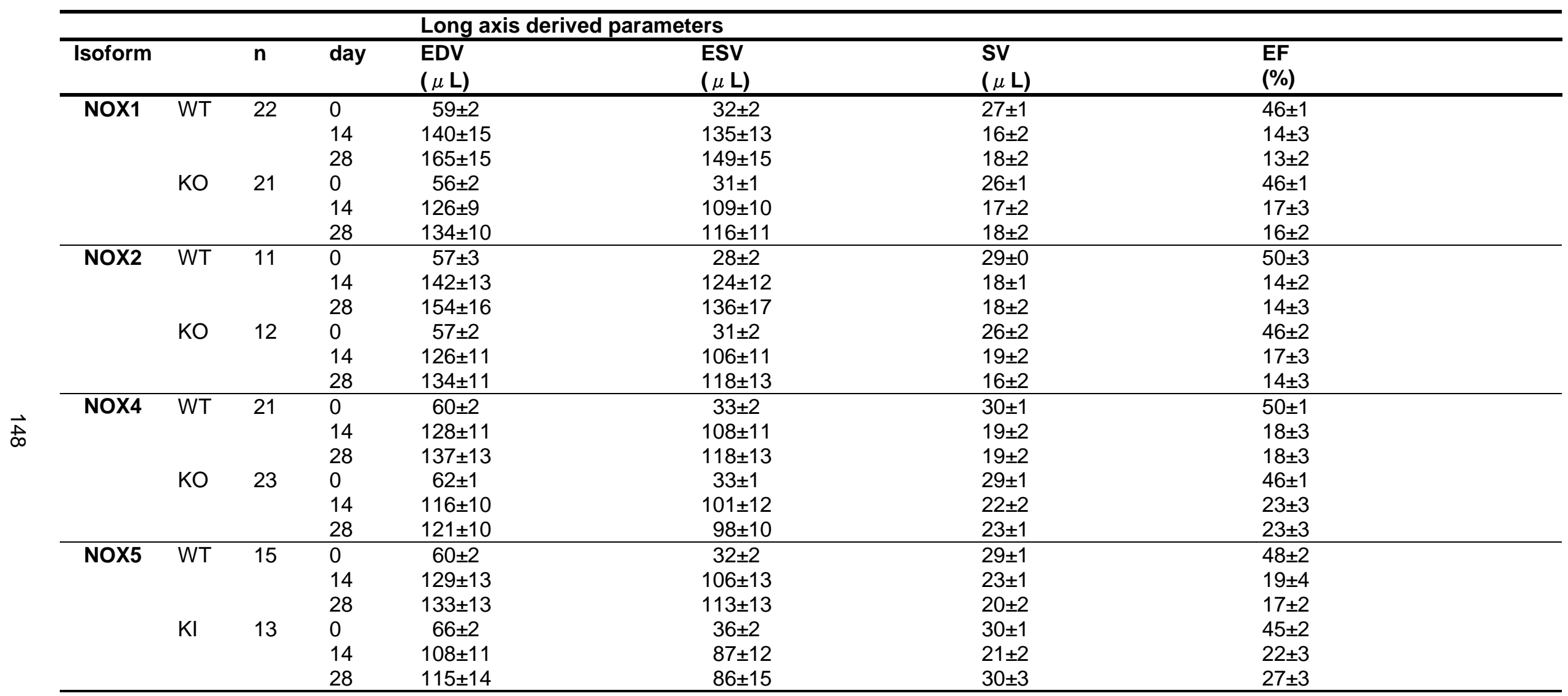


Table 3, continued

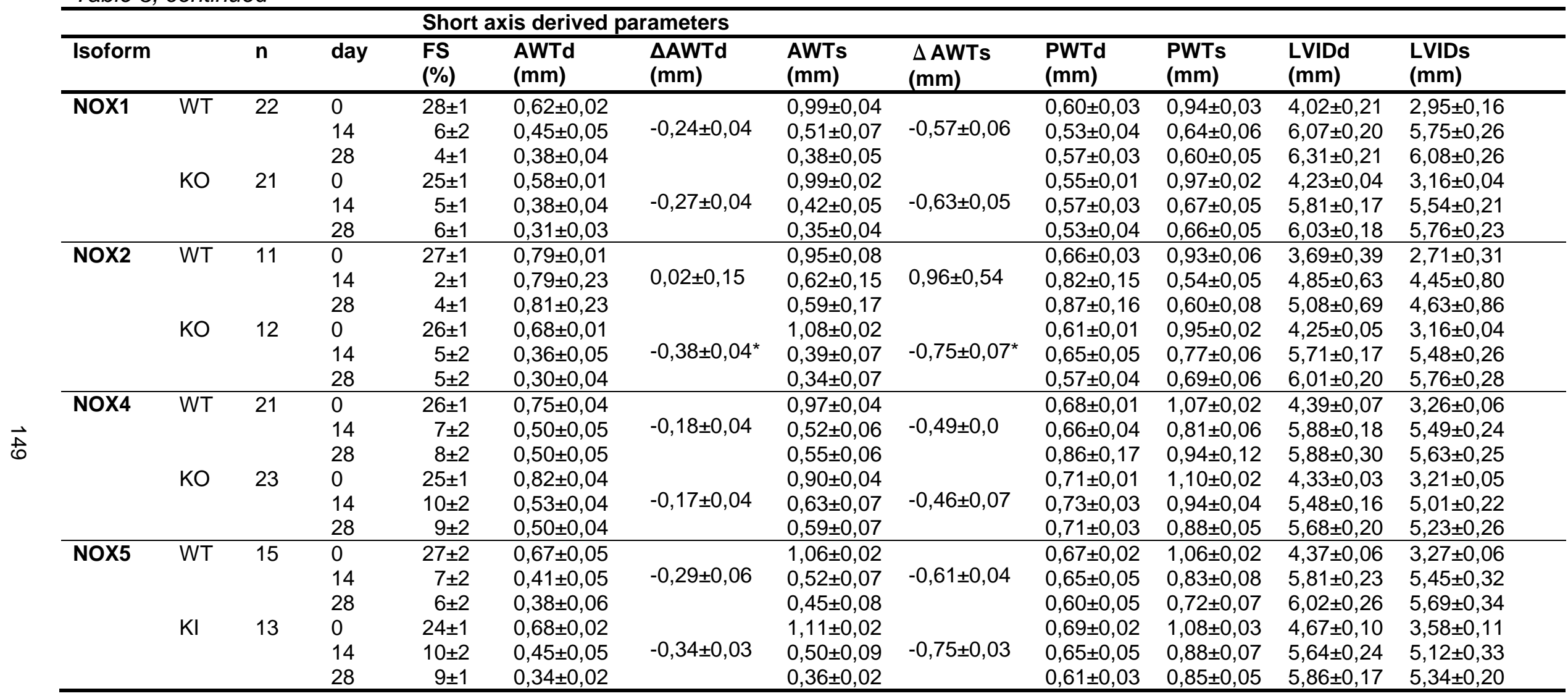

EDV: end diastolic volume; ESV: end systolic volume; SV: stroke volume; EF: ejection fraction; FS: fractional shortening; AWTd/s: left ventricle anterior wall thickness in diastole and systole; PWTd/s: left ventricular posterior wall thickness in diastole and systole; $\triangle A W T d / s:$ change in wall thickness end-basal, representing thinning of the wall) in diastole and systole LVIDd/s: left ventricular internal diameter in diastole and systole * $p<0.05$ vs respective WT. 
Table 4. Ultrasound measurements in the ischemia reperfusion groups.

\begin{tabular}{|c|c|c|c|c|c|c|c|}
\hline & & & & \multicolumn{4}{|c|}{ Long axis derived parameters } \\
\hline \multicolumn{2}{|l|}{ Isoform } & $\mathbf{n}$ & day & $\begin{array}{l}\text { EDV } \\
(\mu \mathrm{L})\end{array}$ & $\begin{array}{l}\text { ESV } \\
(\mu \mathrm{L})\end{array}$ & $\begin{array}{l}\text { SV } \\
(\mu \mathbf{L})\end{array}$ & $\begin{array}{l}\text { EF } \\
\text { (\%) }\end{array}$ \\
\hline \multirow[t]{6}{*}{ NOX1 } & WT & 22 & 0 & $63 \pm 3$ & $33 \pm 3$ & $29 \pm 1$ & $47 \pm 2$ \\
\hline & & & 14 & $74 \pm 5$ & $51 \pm 6$ & $23 \pm 1$ & $34 \pm 3$ \\
\hline & & & 28 & $79 \pm 5$ & $48 \pm 5$ & $25 \pm 2$ & $36 \pm 3$ \\
\hline & KO & 21 & 0 & $63 \pm 2$ & $33 \pm 2$ & $30 \pm 2$ & $48 \pm 2$ \\
\hline & & & 14 & $71 \pm 3$ & $44 \pm 3$ & $27 \pm 2$ & $39 \pm 2$ \\
\hline & & & 28 & $66 \pm 5$ & $41 \pm 4$ & $24 \pm 2$ & $35 \pm 3$ \\
\hline \multirow[t]{6}{*}{ NOX4 } & WT & 21 & 0 & $59 \pm 3$ & $31 \pm 2$ & $28 \pm 2$ & $47 \pm 2$ \\
\hline & & & 14 & $65 \pm 3$ & $40 \pm 4$ & $25 \pm 1$ & $40 \pm 3$ \\
\hline & & & 28 & $69 \pm 3$ & $45 \pm 3$ & $24 \pm 2$ & $36 \pm 2$ \\
\hline & $\mathrm{KO}$ & 23 & 0 & $61 \pm 2$ & $32 \pm 2$ & $29 \pm 1$ & $48 \pm 1$ \\
\hline & & & 14 & $67 \pm 3$ & $39 \pm 2$ & $28 \pm 2$ & $42 \pm 2$ \\
\hline & & & 28 & $72 \pm 3$ & $43 \pm 2$ & $28 \pm 2$ & $39 \pm 2$ \\
\hline \multirow[t]{6}{*}{ NOX5 } & WT & 15 & 0 & $63 \pm 3$ & $33 \pm 3$ & $29 \pm 1$ & $47 \pm 2$ \\
\hline & & & 14 & $74 \pm 5$ & $51 \pm 6$ & $23 \pm 1$ & $34 \pm 3$ \\
\hline & & & 28 & $79 \pm 5$ & $48 \pm 5$ & $25 \pm 2$ & $36 \pm 3$ \\
\hline & $\mathrm{KI}$ & 13 & 0 & $63 \pm 2$ & $33 \pm 2$ & $30 \pm 2$ & $48 \pm 2$ \\
\hline & & & 14 & $71 \pm 3$ & $44 \pm 3$ & $27 \pm 2$ & $39 \pm 2$ \\
\hline & & & 28 & $66 \pm 5$ & $41 \pm 4$ & $24 \pm 2$ & $35 \pm 3$ \\
\hline
\end{tabular}


Table 4, continued

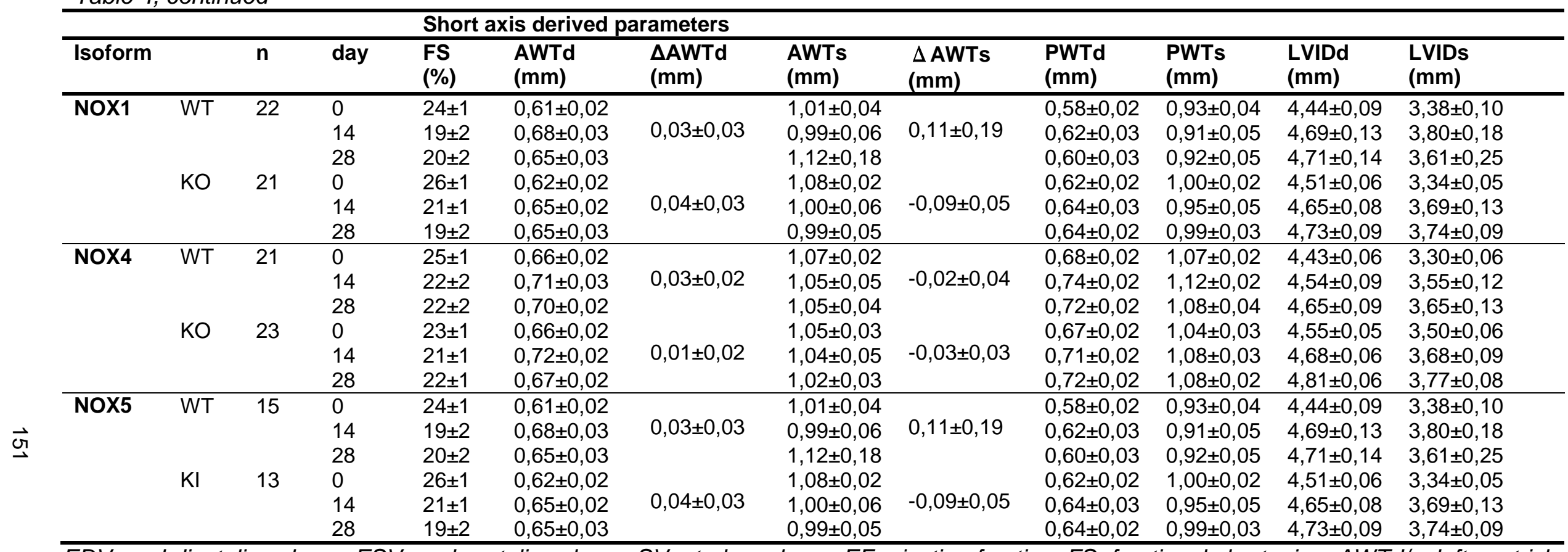

EDV: end diastolic volume; ESV: end systolic volume; SV: stroke volume; EF: ejection fraction; FS: fractional shortening; AWTd/s: left ventricle anterior wall thickness in diastole and systole; PWTd/s: left ventricular posterior wall thickness in diastole and systole; LVIDd/s: left ventricular internal diameter in diastole and systole $\triangle A W T d / s$ : change in wall thickness (end-basal, representing thinning of the wall) in diastole and systole. 
A

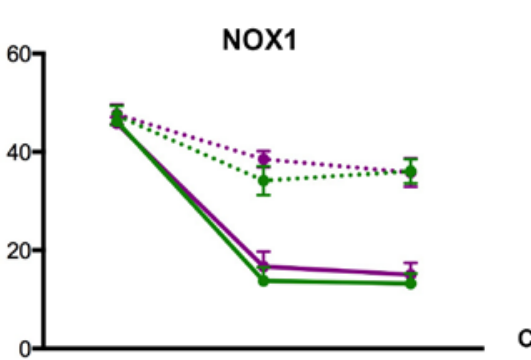

B

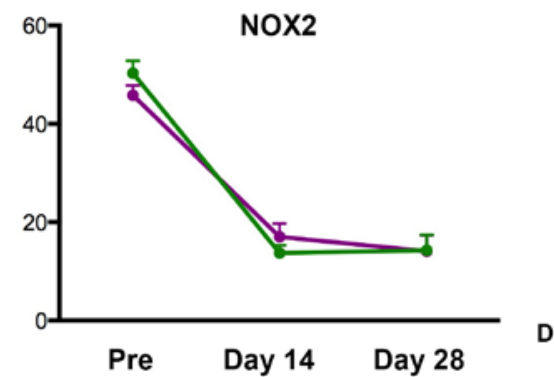

NOX4

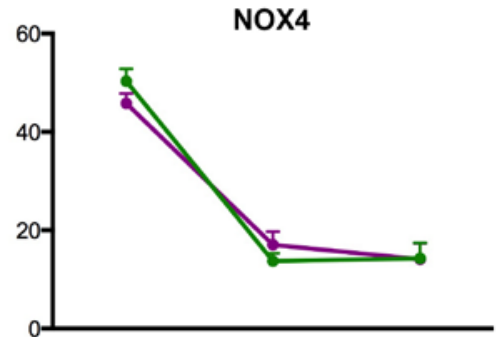

NOX5

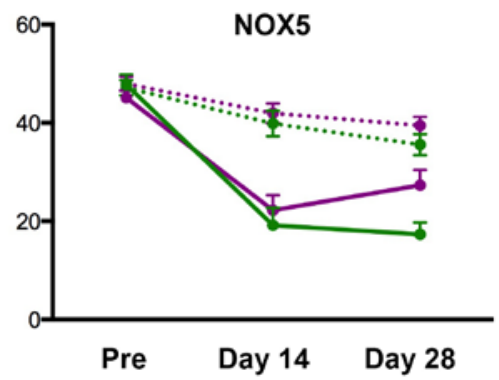

Figure 5. Ejection fraction decreased more after MI (full lines) than after IR (dashed lines), with no significant changes between any of the KO/KI (purple) mice versus their respective WT (green). A) NOX1 KO (MI $n=21, I R n=16)$ and WT (MI $n=22, I R n=18)$. B) NOX2 KO (MI $n=12)$ and $W T(M I n=11)$. C) NOX4 KO (MI $n=23$, IR $n=15)$ and $W T(M I n=21, I R n=14)$.

D) NOX5 KI (MI $n=13, I R n=18)$ and $W T$ mice(MI $n=15$, IR $n=18)$. EF was measured by ultrasound at day 0,14 and 28 and presented as mean \pm SEM.

In addition to the ultrasounds, at the end of the experiments, just before sacrificing the mice, pressures and contractility of the heart were measured in vivo in response to infusion of increasing concentrations of dobutamine to stimulate maximum contractility (Fig. 6).

After MI, NOX4 KO mice $(5,6 \pm 0,5 \mathrm{mmHg}, \mathrm{n}=22)$ showed an increased diastolic pressure compared to the NOX4 WT mice $(3,6 \pm 0,8 \mathrm{mmHg}, \mathrm{n}=20)$ and an increased

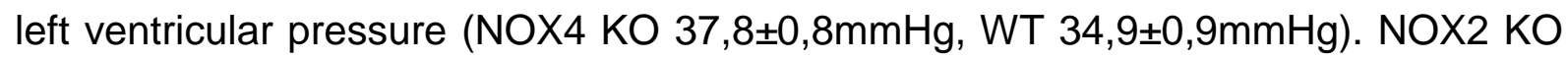
mice subjected to permanent ligation of the LAD showed an increase in left

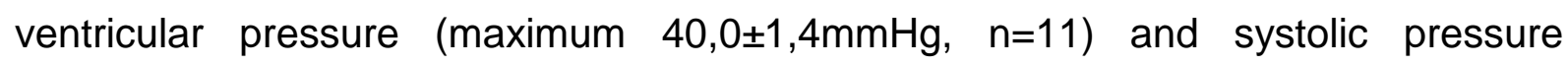
(maximum $97,9 \pm 1,1 \mathrm{mmHg}, \mathrm{n}=11$ ) when compared to their matched WT's (LVP $34,4 \pm 1,3 \mathrm{mmHg}$; systolic $89,9 \pm 2,7 \mathrm{mmHg}, \mathrm{n}=9$ ). 
However, the power of these results is very low because of the low n number (additional experiments currently being performed).

NOX5 KI mice with a myocardial infarction showed an increased contractility (max $12626 \pm 545 \mathrm{mmHg} / \mathrm{s}, \mathrm{n}=16)$ and enhanced relaxations $(-7566 \pm 208 \mathrm{mmHg} / \mathrm{s})$ compared to the WT mice $(10489 \pm 646 \mathrm{mmHg} / \mathrm{s}$ and $-6665 \pm 367 \mathrm{mmHg} / \mathrm{s}$ respectively, $\mathrm{n}=14)$.

\section{Oxidative stress similar in NADPH oxidase $K O$ and WT mice subjected to ischemia-reperfusion or permanent ischemia of the heart}

DHE staining was used to measure the total amount of oxidative stress in cryosections of the left ventricle (Fig. 7). Myocardial infarction was associated with less oxidative stress than ischemia-reperfusion, but not significantly.

None of the NADPH oxidases affected the amount of oxidative stress in either of the models (Fig.7, Table 2).

\section{Discussion}

We extensively studied the role of all vascular NADPH oxidases in long term cardiac remodelling after $\mathrm{MI}$ or IR. This is to our knowledge the first study that compares all vascular NOX isoforms to their respective WT's in a permanent and transient ischemic model of the heart up to 4 weeks.

We found no role of NOX4 in the chronic response to MI or IR in the heart using the NOX4Ma mice. Other data on NOX4 in ischemia reperfusion injury of the heart have only been described for short-term experiments. Although 1 group showed no role ${ }^{7}$, another group showed smaller infarcts in both global and cardiac specific NOX4 KO mice ${ }^{6}$. In the same study, transgenic NOX4 mice, overexpressing NOX4 specifically in cardiomyocytes, did not show different responses to heart ischemia than nontransgenic mice ${ }^{6}$. Thus a possible role of NOX4 in heart ischemia is not straightforward. NOX2/cardiac specific NOX4 double KO mice even showed larger infarct sizes than WT mice ${ }^{6}$. This last study could indicate that there are complex interplays between the different NOX isoforms and inhibiting one could results in upor downregulation of others. 


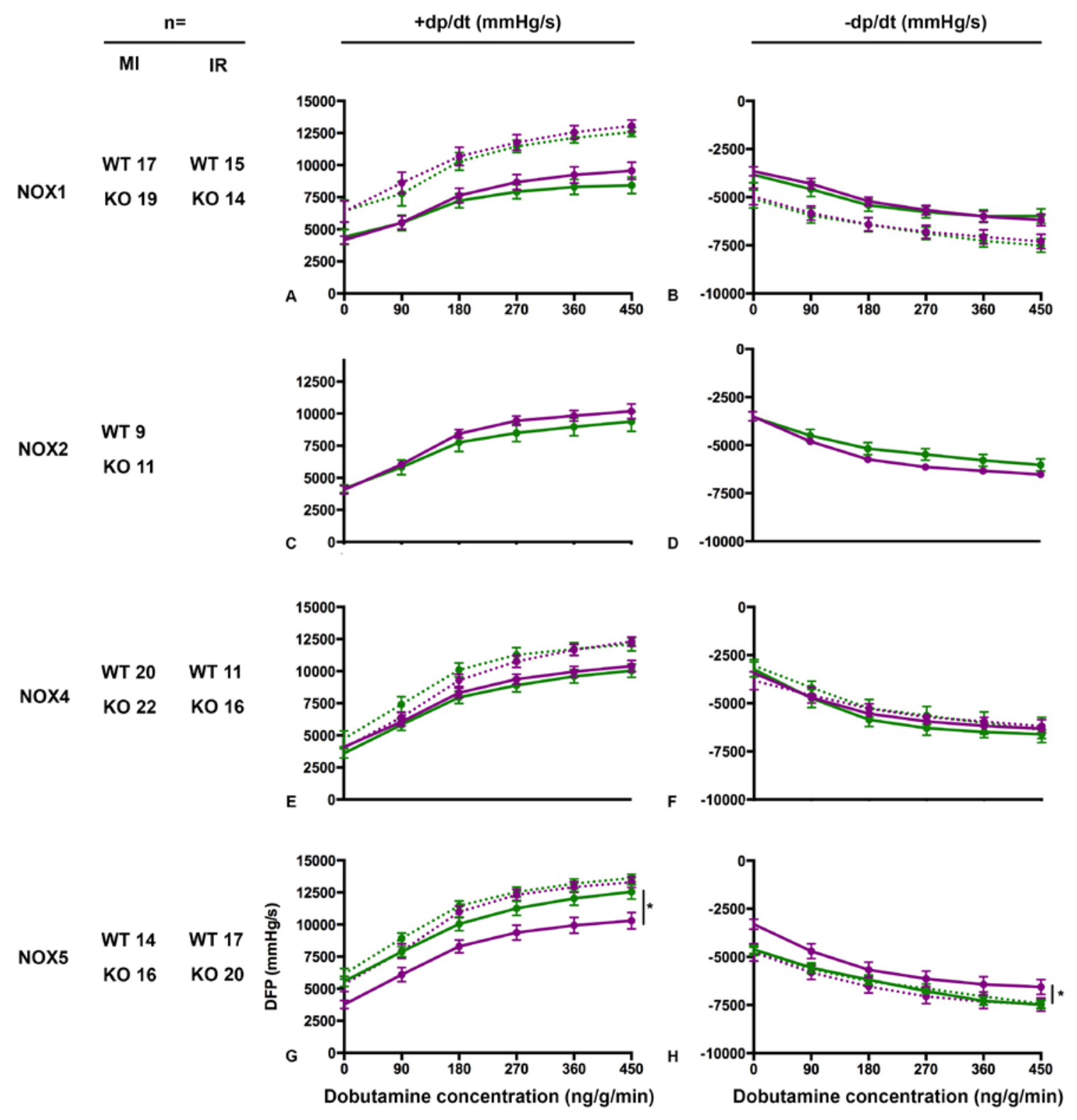

Figure 6. Left ventricular function was better preserved 4 weeks after IR (dashed lines) versus $M I$ (full lines). $A, C, E, G)$ Change in cardiac contractility ( $+d p / d t)$ during dobutamine stimulation for the different WT (green) and KO/Ki (purple) mice. B,D,F,H) Change in cardiac relaxation $(-d p / d t)$ during dobutamine stimulation for the different $W T$ (green) and $K O / K I$ (purple) mice. Data points are presented as mean \pm SEM. * $p<0.05 \mathrm{KO} / \mathrm{KI}$ versus $W T$. 

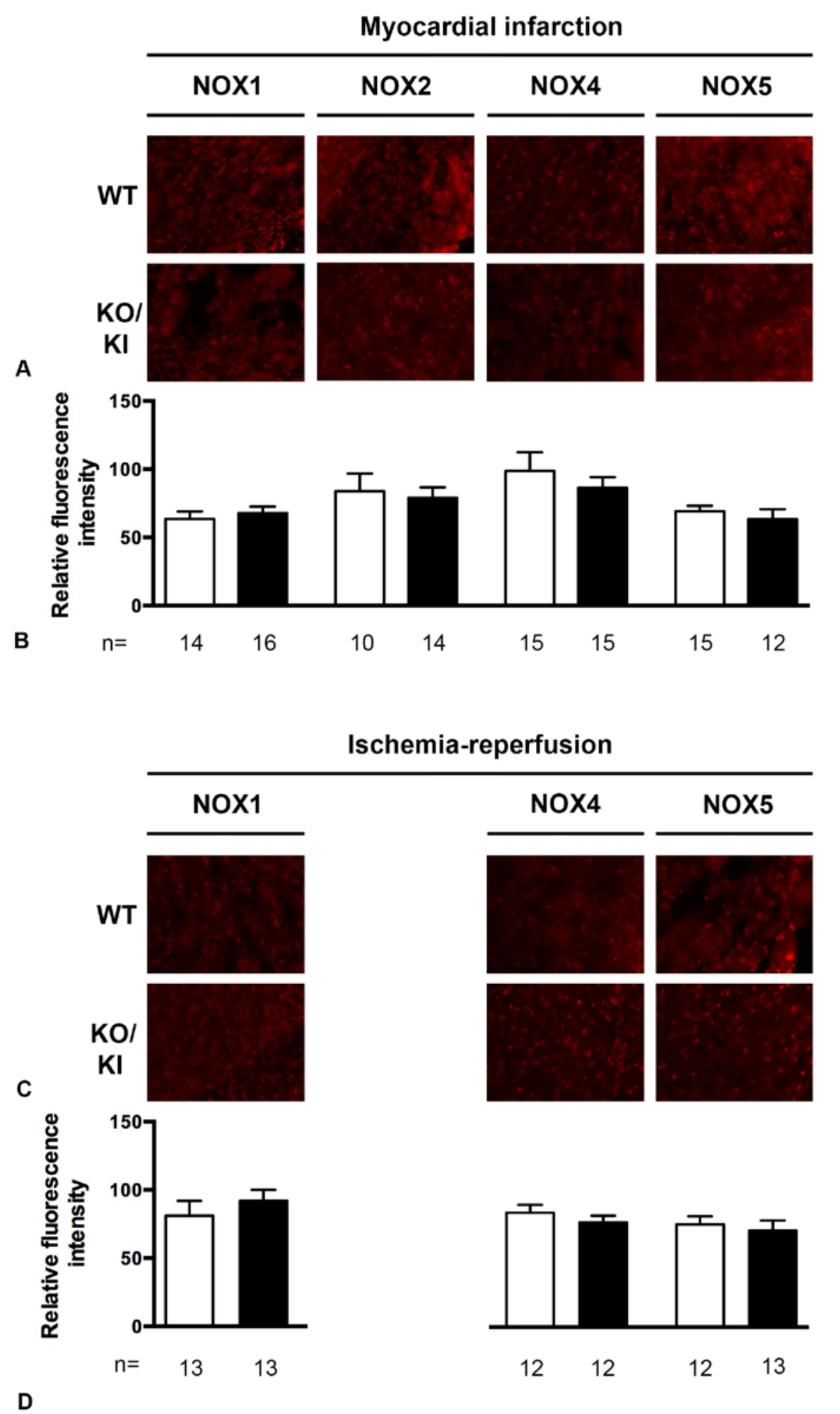

Figure 7. None of the NADPH oxidases affected oxidative stress in the left ventricle 4 weeks after MI or IR. A,C) Representative pictures of DHE staining in the $M I(A)$ and IR (C) groups. $B, D)$ Average intensity of nuclear staining expressed as mean \pm SEM in WT (white bars) and KO/KI (black bars) mice. 
So far, we have not checked for this up- or downregulation in our models. Discrepancies between the different studies could be explained also by the use of different $\mathrm{KO}$ strategies of the global NOX4 KO mice or differences in surgical models or anaesthesia. In addition, since cardiac specific KO's show differences with global KO's, cell type specificity might play a role and needs to be investigated further. In the near future, these data using the NOX4Ma mice will be validated by repeating these experiments using the NOX4Wü or NOX4Me mice.

In the NOX1 group both for the permanent and transient ischemia, no differences in infarct size, collagen content, functionality or heart dimensions were found. The only small finding was a decreased heart weight in the $\mathrm{KO}$ mice after myocardial infarction but as this is not accompanied by other changes in functionality or infarct size, no major role for NOX1 is suggested by our study. The NOX2 KO mice with a myocardial infarction showed an increased collagen content in the infarcted area and more thinning of the anterior ventricle wall. Left ventricular and systolic pressure during dobutamine stimulation were increased also. These findings suggest that NOX2 might have a small role in wound healing and thus in ventricular remodelling after permanent ischemia of the heart. Components of the wound healing might be different, but this did not affect functionality. Several other papers have described the role of NOX1 and 2 in ischemia(-reperfusion) of the heart (Table 5), showing a complex picture.

On the short term, after transient ischemia, no role was found for the subunit p4 $7^{\text {phox } 19}$, while 1 study indicates a possible small role for NOX1 after ischemiareperfusion injury of the heart but again no role after permanent ischemia ${ }^{7}$. This difference could be explained by the fact that with permanent ischemia, no oxygen is present to be formed into detrimental ROS. Also, the difference in KO strategy (p47 $7^{\text {phox }}$ or NOX1) might result in different findings. In the IR study, only an effect on infarct size was found (40\% decrease) but other measures such as oxidative stress and neutrophil influx were not different in NOX1 KO mice.

More importantly, functional parameters, which are more clinically relevant, were not assessed in this study. One other study shows a decrease in fibrosis and better left ventricular function in $\mathrm{p} 47^{\text {phox }} \mathrm{KO}$ mice. This is in contrast with our own study, but again there mouse models cannot be easily compared. 
Although NOX1 might thus play a minor role in the acute phase after IR, the longterm outcome is not altered thus deleting also NOX1 from our possible therapeutically target list in ischemic heart disease.

The picture for NOX2 on the short term seems more clear, with 2 studies showing a detrimental effect on infarct size and apoptosis after ischemia reperfusion injury ${ }^{6,7}$. However, more clinically relevant functional changes were again not assessed. Our own study shows a possible beneficial role of NOX2 after myocardial infarction on the long term. Remodelling seems to be affected with more collagen formation and higher left ventricular pressure in the NOX2 KO mice. Functionality and dimensions of the heart were not changed. The found changes were rather small for the amount of animals used and are thus underpowered (additional experiments are currently being done). Our data conflict with three other studies that show either a detrimental role of NOX2 in myocardial infarction ${ }^{16}$ or no role at all ${ }^{15,17}$.

The study showing the detrimental role did not show a change in infarct size and the functional changes are rather small. Combining our data with the other known literature, looking especially at the more clinically relevant long-term outcome, NOX2 is neither a good treatment target in heart IRI.

In humans, NOX5 has been shown to be involved in oxidative stress in coronary artery disease ${ }^{20}$ and to be upregulated after myocardial infarction ${ }^{21}$. In our humanised NOX5 KI mice, we found a better preserved contractility and enhanced relaxation after dobutamine stimulation in the myocardial infarction group. This would suggest that NOX5 is beneficial in the chronic phase after a myocardial infarction. However, no changes in infarct size, collagen content or ejection fraction were seen. Since NOX5 is normally expressed in humans and even upregulated after a myocardial infarction, a possible therapeutic approach would be to stimulate NOX5. Overproduction of ROS however, could lead to a damaging effect by deregulation of the oxidative/reductive balance, leaving NOX5 as a possible treatment target but with major limitations. 
Table 5. Overview of the studies on NOX in ischemia(-reperfusion) of the heart.

\begin{tabular}{|c|c|c|c|c|c|c|c|}
\hline Isoform & $\begin{array}{l}\text { Mouse } \\
\text { model }\end{array}$ & MI / IR & $\begin{array}{c}\text { Timing } \\
\text { outcome }\end{array}$ & $\begin{array}{c}\text { Infarct } \\
\text { size }\end{array}$ & Function & $\begin{array}{c}\text { Collagen } \\
\text { deposition }\end{array}$ & Ref \\
\hline \multirow[t]{3}{*}{ NOX1 } & 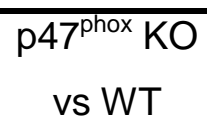 & IR & $24 h$ & $\varnothing$ & $\varnothing$ & n.a & 19 \\
\hline & $\begin{array}{l}\text { NOX1 KO } \\
\text { vs WT }\end{array}$ & $\begin{array}{l}\mathrm{IR} \\
\mathrm{MI}\end{array}$ & $24 \mathrm{~h}$ & $\begin{array}{l}\downarrow \\
\varnothing\end{array}$ & $\begin{array}{l}\text { n.a } \\
\text { n.a }\end{array}$ & $\begin{array}{l}\text { n.a } \\
\text { n.a }\end{array}$ & 7 \\
\hline & $\begin{array}{c}\text { NOX1 KO } \\
\text { vs WT } \\
\text { p47 } 7^{\text {phox }} \text { KO } \\
\text { vs WT }\end{array}$ & $\begin{array}{l}\mathrm{IR} \\
\mathrm{MI} \\
\mathrm{MI}\end{array}$ & $\begin{array}{l}28 \text { days } \\
30 \text { days }\end{array}$ & $\begin{array}{l}\varnothing \\
\varnothing \\
\varnothing\end{array}$ & $\begin{array}{l}\varnothing \\
\varnothing \\
\uparrow\end{array}$ & $\begin{array}{l}\varnothing \\
\varnothing \\
\downarrow\end{array}$ & * \\
\hline \multirow[t]{5}{*}{ NOX2 } & $\begin{array}{c}\text { NOX2 KO } \\
\text { vs WT } \\
\text { NOX2 KO } \\
\text { vs WT }\end{array}$ & $\begin{array}{l}\text { IR } \\
\mathrm{MI}\end{array}$ & $24 h$ & $\begin{array}{l}\downarrow \\
\varnothing\end{array}$ & $\begin{array}{l}\text { n.a } \\
\text { n.a }\end{array}$ & $\begin{array}{l}\text { n.a } \\
\text { n.a }\end{array}$ & 7 \\
\hline & $\begin{array}{c}\text { NOX2 KO } \\
\text { vs WT }\end{array}$ & $\mathrm{Ml}$ & 8 weeks & $\varnothing$ & $\varnothing$ & $\varnothing$ & 15 \\
\hline & $\begin{array}{c}\text { NOX2 KO } \\
\text { vs WT }\end{array}$ & $\mathrm{Ml}$ & 4 weeks & $\varnothing$ & $\uparrow$ & $\downarrow$ & 16 \\
\hline & $\begin{array}{c}\text { NOX2 KO } \\
\text { vs WT }\end{array}$ & $\mathrm{MI}$ & $\begin{array}{c}\text { 3/7/14/28 } \\
\text { days }\end{array}$ & $\varnothing$ & n.a & $\varnothing$ & 17 \\
\hline & $\begin{array}{c}\text { NOX2 KO } \\
\text { vs WT }\end{array}$ & $\begin{array}{l}\mathrm{MI} \\
\mathrm{IR}\end{array}$ & 28 days & $\begin{array}{l}\varnothing \\
\varnothing\end{array}$ & $\begin{array}{l}\varnothing \\
\varnothing\end{array}$ & $\begin{array}{c}\uparrow \text { (in infarct } \\
\text { area) }\end{array}$ & * \\
\hline \multirow[t]{5}{*}{ NOX4 } & $\begin{array}{c}\text { NOX4 KO } \\
\text { vs WT }\end{array}$ & IR & $24 \mathrm{~h}$ & $\varnothing$ & n.a & n.a & 7 \\
\hline & $\begin{array}{c}\text { cNOX4 } \\
\text { KO vs WT }\end{array}$ & IR & $24 \mathrm{~h}$ & $\downarrow$ & n.a & n.a & 6 \\
\hline & $\begin{array}{c}\text { gNOX4 } \\
\text { KO vs WT }\end{array}$ & & & $\downarrow$ & n.a & n.a & \\
\hline & Tg NOX4 & & & $\varnothing$ & n.a & n.a & \\
\hline & $\begin{array}{c}\text { NOX4 KO } \\
\text { vs WT }\end{array}$ & $\begin{array}{l}\mathrm{MI} \\
\mathrm{IR}\end{array}$ & 28 days & $\begin{array}{l}\varnothing \\
\varnothing\end{array}$ & $\begin{array}{l}\varnothing \\
\varnothing\end{array}$ & $\begin{array}{l}\varnothing \\
\varnothing\end{array}$ & * \\
\hline
\end{tabular}

cNOX4 KO, cardiac NOX4 KO; gNOX4 KO, global NOX4 KO; Tg NOX4, transgenic NOX4; $I R$, ischemia reperfusion; MI, myocardial infarction; n.a., not applicable; $\varnothing$, no change;

${ }^{*}$ current study. 
In conclusion, in our view NADPH oxidases are no major targets in ischemic heart disease. Time dependent differences (acute versus long-term), the presence or absence of reperfusion and possible cell specific effects create a complex process in which the KO of $1 \mathrm{NADPH}$ oxidase is not important. In addition, the complex interplay between different NOX isoforms and other pro- and antioxidant enzymes add an additional degree of complexity.

\section{Methods}

\section{Animals}

All animal experiments were approved by the local animal experimental committee and performed according to the Dutch law on animal experiments. Animals were housed under controlled conditions $\left(22^{\circ} \mathrm{C}, 55-65 \%\right.$ humidity, $12 \mathrm{~h}$ light-dark cycle), and were allowed free access to water and standard laboratory chow. Male and female animals aged 8-12 weeks were used. The NOX1 KO, NOX 2KO, NOX 4 KO and NOX5 KI animals were compared to their respective matched WT's.

\section{Myocardial ischemia-reperfusion}

After administration of an analgetic (buprenorphine s.c. $0,05 \mathrm{mg} / \mathrm{kg}$, Temgesic, Schering-Plough), mice were anaesthetized with isoflurane (Abbott forene Isoflurane) $4-5 \%$ in air and intubated per orally with a stainless-steel tube.

The tube was connected to a respirator (rodent ventilator Microvent type 845, Hugo Sachs Electronic, Germany), set at a stroke volume of $250 \mu \mathrm{L}$ and a rate of 210 strokes/min. Anaesthesia was then maintained with $2-3 \%$ isoflurane in air via a vaporizor (Univentor, UNO Roestvaststaal BV) connected to the respirator.

The mouse was placed on a heating pad (UNO temperature control unit, UNO Roestvaststaal BV) and body temperature was monitored using a rectal probe and maintained at $37.0^{\circ} \mathrm{C}$ using a feedback-controlled infrared light. During surgery, an ECG was recorded with IDEEQ software (IDEE, Maastricht University).

A left thoracotomy was performed to expose the heart. Then, the left descending coronary artery (LAD) was ligated with a 6-0 polypropylene suture (Surgipo, Chicago, IL, USA), just proximal to its main branching point. 
The suture was tied around a $3 \mathrm{~mm}$-long polyethylene tube (PE-10) to induce ischemia. Ischemia was assessed by a discolouration of the tissue (from red to pinkwhite) and ST-elevation on ECG. After 45 minutes, the blood flow was re-established by removal of the polyethylene-tube. The occurrence of reperfusion was assessed by the colour of the tissue turning red again. For the myocardial infarct model, the LAD was ligated with a 6-0 polypropylene suture permanently.

The chest was closed with 5-0 silk sutures (Ethicon). The animals were weaned from the respirator and the endotracheal tube was removed, once the mice breathed spontaneously. After surgery, mice were allowed to recover at thermoneutral temperature $\left(28^{\circ} \mathrm{C}\right)$. In the afternoon and the morning the day after, s.c. $0.05 \mathrm{mg} / \mathrm{kg}$ buprenorphine hydrochloride was repeated to relieve pain.

\section{Echocardiography}

In vivo echocardiography measurements were performed under light isoflurane anaesthesia before the surgery and at day 14 and 28, using the Vevo 2100 echocardiography system (Visualsonics, Toronto, Canada). Two-dimensional BMode echocardiograms were captured at a rate of $90-120 \mathrm{~Hz}$ from parasternal longaxis views as well as from mid-papillary short axis-views of the left ventricle. Data were obtained from at least 3 different images taken in end-diastole and systole using the accompanying software from the Vevo 2100 echocardiography system.

From the long-axis echocardiograms, the LV area in diastole and systole (LVAd and LVAs), as well as the length of the LV lumen from base to apex in diastole and systole (LVLd and LVLs) were determined. From these parameters, the end-diastolic (EDV) and end-systolic (ESV) volumes were calculated using the following formulas: $8^{*}\left(\right.$ LVAd $^{2} / 3 \pi$ LVLd and $8^{*}(\text { LVAs) })^{2} / 3 \pi$ LVLs. Stroke volume (SV) was determined as EDV-ESV and the ejection fraction (EF) was defined as $100 *((E D V-E S V) / E D V){ }^{23-25}$. From the short axis echocardiograms, the LV internal chamber diameters in diastole and systole were determined: LVIDd and LVIDs, respectively. The LV fractional shortening (FS) was calculated from these parameters according to the following formula: $100 *(($ LVIDd-LVIDs)/LVIDd). 


\section{Evaluation of left ventricular contractility}

Left ventricular contractility was measured at day 28 before sacrifice. Mice were anaesthetized with urethane $2.5 \mathrm{mg} / \mathrm{kg}$ intraperitoneally (Sigma). Body temperature and respiration were controlled as described above.

A high-fidelity pressure transducer (Mikro-tip, 1.4F, SPR-671 Millar Instruments, Houston, TX, USA) was inserted into the left ventricle via the right carotid artery.

Ventricular pressure was measured and sampled at a rate of $2 \mathrm{kHz}$. After a baseline measurement, the heart was stimulated by intravenous infusion of increasing doses of dobutamine (Sigma) via a microinjection pump (Model 200 series, KdScientific, Boston, MA, USA) starting at $90 \mathrm{ng} / \mathrm{min}$ and increasing by $90 \mathrm{ng}$ every two minutes to a maximum dose of $540 \mathrm{ng} / \mathrm{min}$. Heart rate, maximal positive pressure and minimal positive pressure were calculated for every infusion rate using IdeeQ software.

\section{Tissue sampling}

After the hemodynamic measurements at day 28 , the thorax of the mice was opened, the heart was quickly excised and the atria were removed. The ventricles were cut transversally at $3 \mathrm{~mm}$ from the apex. The apical part was fixed in formalin and processed for paraffin embedding. The basal part was longitudinally cut in two, one part was shock frozen in liquid nitrogen, one part was embedded in OCT (TissueTek).

\section{Evaluation of infarct size}

Infarct sizes were calculated using AZAN stained paraffin sections. Formalin-fixed, paraffin embedded sections of $4 \mu \mathrm{m}$ were quickly rehydrated. Slides were incubated in preheated AZAN I solution for 30 minutes at $37^{\circ} \mathrm{C}$, followed by rinsing in demineralised water and incubation in 5\% phosphotungstic acid for 45 minutes. After rinsing in tap water, slides were finally incubated in AZAN II solution (diluted 1:3 in demineralised water) for 10 minutes, dehydrated, mounted with Entellan and coverslipped. Pictures were taken using a Leica camera connected to a Zeiss microscope. Pictures were analysed using the Leica Qwin pro v3.5.1 software. Infarct sizes are expressed as percentage area of the total left ventricular tissue area. 


\section{Collagen content determination}

To determine the collagen content 4 weeks after MI or IR of the heart, paraffinsections were stained with Picrosirius red. Formalin-fixed, paraffin embedded sections of $4 \mu \mathrm{m}$ were quickly deparaffinised, rehydrated and incubated in $0.2 \%$ phosphomolybdic acid hydrate for 5 minutes. After incubation in $0.1 \%$ picrosirius red in saturated picric acid $(\mathrm{pH} 2.0)$ for 90 minutes and $0.01 \mathrm{~N} \mathrm{HCl}$ for 2 minutes, slides were rinsed in $70 \%$ alcohol for 45 seconds, followed by dehydration, mounting with Entellan and coverslipping. Pictures were taken using a Leica camera connected to a Zeiss microscope. Pictures were analysed using the Leica Qwin pro v3.5.1 software. For each sample, 2 pictures were taken in the infarct zone, the border zone and the septum each. Collagen content is expressed as percentage of the total area within the specified region.

\section{Oxidative stress}

The amount of general ROS production was measured using dihydroethidium (DHE, Sigma Aldrich, D7008, lot no SLBD6013V) dissolved in PBS. Cryosections of $7 \mu \mathrm{m}$ were incubated in $2 \mu \mathrm{M}$ DHE for 5 minutes at room temperature. Fluorescence intensity was then measured using a Zeiss filter (excitation 510nm, emission 590nm) and a fluorescent light.

Three random pictures of each sample were taken using a Leica camera connected to a Zeiss microscope. Mean fluorescence intensity of the nuclei was measured using the Leica Qwin pro v3.5.1 software.

\section{Statistics}

All data are expressed as mean \pm SEM. Using the GraphPad Prism 5.0 software package statistical differences between mean values were determined by Student's two-tailed t-test. For repeated measurements (ultrasound, hemodynamic measurements), a two-way ANOVA was used. Mortality was analysed using the Kaplan Meier Survival curve. A value of $p<0.05$ was considered to be statistically significant. 


\section{Power}

To ensure sufficient statistical power $(>80 \%)$ for our experiments, the number of animals needed per group was calculated for the most relevant, i.e. primary outcome, hemodynamic improvement. Based on an alpha of 0.05, a detectable difference of at least $40 \%$ and a standard deviation of $25 \%$ (based on our previous experience with this model), $\mathrm{n}=7$ animals per group were needed to ensure a power of $80 \%$ according to Sachs' formula (Sachs, Angewandte Statistik). Post hoc analysis showed that the estimated variance was around $25 \%$ for the Ml groups and even much lower (around 12\%) for the IR group, ensuring a power of $>80 \%$ for all groups (Table 6).

Table 6. Power analysis.

\begin{tabular}{|c|c|c|c|c|c|c|c|c|c|c|}
\hline \multirow{3}{*}{$\begin{array}{l}\mathrm{MI} / \\
\mathrm{IR}\end{array}$} & \multirow[t]{3}{*}{ Isoform } & \multicolumn{8}{|c|}{ Maximum contractility (mmHg/s) } & \multirow{3}{*}{$\begin{array}{l}\text { Power for } \\
40 \% \text { effect }\end{array}$} \\
\hline & & \multicolumn{4}{|c|}{ WT } & \multicolumn{4}{|c|}{$\mathrm{KO} / \mathrm{KI}$} & \\
\hline & & $\mathrm{N}$ & Mean & SD & CV & $\mathrm{N}$ & Mean & SD & CV & \\
\hline \multirow[t]{4}{*}{$\mathrm{Ml}$} & NOX1 & 16 & 8334 & 2413 & 0,29 & 22 & 9240 & 2983 & 0,32 & 99,7 \\
\hline & NOX2 & 10 & 9267 & 2552 & 0,28 & 11 & 10497 & 1946 & 0,19 & 93,5 \\
\hline & NOX4 & 20 & 10382 & 2437 & 0,23 & 23 & 10407 & 2289 & 0,22 & 99,9 \\
\hline & NOX5 & 14 & 10489 & 2417 & 0,23 & 16 & 12626 & 2182 & 0,17 & 98,8 \\
\hline \multirow[t]{3}{*}{ IR } & NOX1 & 19 & 12342 & 1763 & 0,14 & 14 & 13381 & 1594 & 0,12 & 100 \\
\hline & NOX4 & 13 & 12487 & 1028 & 0,08 & 16 & 12439 & 1740 & 0,14 & 100 \\
\hline & NOX5 & 18 & 13414 & 2125 & 0,16 & 20 & 13837 & 1378 & 0,10 & 100 \\
\hline
\end{tabular}

To assess the power of our studies, the pooled variance for the $M I$ experiments and the $I R$ experiments were calculated separately. These pooled variances were used to calculate the power for detecting a $40 \%$ difference using the Russ Lenth's power and sample size software (http://homepage.stat.uiowa.edu/ rlenth/Power/index.html). CV, coefficient of variations (=SD/mean); $S D$, standard deviation.

\section{References}

1. World Health Organisation. Global Atlas on cardiovascular disease prevention and control 2011. 1-164 (2011).

2. Hausenloy, D. J. \& Yellon, D. M. Myocardial ischemia-reperfusion injury: a neglected therapeutic target. J. Clin. Invest. 123, 92-100 (2013).

3. Yellon, D. M. \& Hausenloy, D. J. Myocardial reperfusion injury. N. Engl. J. Med. 357, 1121-1135 (2007). 
4. Zhang, Y., Tocchetti, C. G., Krieg, T. \& Moens, A. L. Oxidative and nitrosative stress in the maintenance of myocardial function. Free Radic Biol Med 53, 1531-1540 (2012).

5. Kleinschnitz, C. et al. Post-stroke inhibition of induced NADPH oxidase type 4 prevents oxidative stress and neurodegeneration. PLoS Biol. 8, (2010).

6. Matsushima, S. et al. Broad Suppression of NADPH Oxidase Activity Exacerbates Ischemia/Reperfusion Injury Through Inadvertent Downregulation of HIF-1 and Upregulation of PPARa. Circ Res 112, -1149 (2013).

7. Braunersreuther, V. et al. Role of NADPH oxidase isoforms NOX1, NOX2 and NOX4 in myocardial ischemia/reperfusion injury. J. Mol. Cell. Cardiol. 64, 99107 (2013).

8. Zhang, M. et al. NADPH oxidase-4 mediates protection against chronic loadinduced stress in mouse hearts by enhancing angiogenesis. Proc Natl Acad Sci U S A 107, 18121-18126 (2010).

9. Kuroda, J. et al. NADPH oxidase 4 (Nox4) is a major source of oxidative stress in the failing heart. Proc Natl Acad Sci U S A 107, 15565-15570 (2010).

10. Datla, S. R. et al. Important role of Nox4 type NADPH oxidase in angiogenic responses in human microvascular endothelial cells in vitro. Arterioscler Thromb Vasc Biol 27, 2319-2324 (2007).

11. Peshavariya, $\mathrm{H}$. et al. NADPH oxidase isoform selective regulation of endothelial cell proliferation and survival. Naunyn Schmiedebergs Arch. Pharmacol. 380, 193-204 (2009).

12. Clempus, R. E. et al. Nox4 is required for maintenance of the differentiated vascular smooth muscle cell phenotype. Arterioscler Thromb Vasc Biol 27, 4248 (2007).

13. Sturrock, A. et al. Transforming growth factor-beta1 induces Nox4 NAD(P)H oxidase and reactive oxygen species-dependent proliferation in human pulmonary artery smooth muscle cells. Am. J. Physiol. Lung Cell Mol. Physiol. 290, L661-L673 (2006).

14. Cucoranu, I. et al. $\mathrm{NAD}(\mathrm{P}) \mathrm{H}$ oxidase 4 mediates transforming growth factorbeta1-induced differentiation of cardiac fibroblasts into myofibroblasts. Circ Res 97, 900-907 (2005). 
15. Frantz, S. et al. Left ventricular remodeling after myocardial infarction in mice with targeted deletion of the NADPH oxidase subunit gp91PHOX. Basic Res. Cardiol. 101, 127-132 (2006).

16. Looi, Y. H. et al. Involvement of Nox2 NADPH oxidase in adverse cardiac remodeling after myocardial infarction. Hypertension 51, 319-325 (2008).

17. Zhao, W., Zhao, D., Yan, R. \& Sun, Y. Cardiac oxidative stress and remodeling following infarction: role of NADPH oxidase. Cardiovasc. Pathol. 18, 156-166 (2009).

18. Doerries, C. et al. Critical role of the $\mathrm{NAD}(\mathrm{P}) \mathrm{H}$ oxidase subunit p47phox for left ventricular remodeling/dysfunction and survival after myocardial infarction. Circ Res 100, 894-903 (2007).

19. Hoffmeyer, M. R. et al. Myocardial ischemia/reperfusion injury in NADPH oxidase-deficient mice. Circ Res 87, 812-817 (2000).

20. Guzik, T. J. et al. Calcium-dependent NOX5 nicotinamide adenine dinucleotide phosphate oxidase contributes to vascular oxidative stress in human coronary artery disease. J. Am. Coll. Cardiol. 52, 1803-1809 (2008).

21. Hahn, N. E. et al. NOX5 expression is increased in intramyocardial blood vessels and cardiomyocytes after acute myocardial infarction in humans. Am. J. Pathol. 180, 2222-2229 (2012).

22. van den Borne, S. W. et al. Mouse strain determines the outcome of wound healing after myocardial infarction. Cardiovasc Res 84, 273-282 (2009).

23. Dodge, H. T., SANDLER, H., BALLEW, D. W. \& LORD, J. D. The use of biplane angiocardigraphy for the measurement of left ventricular volume in man. Am. Heart J. 60, 762-776 (1960).

24. Vine, D. L., Dodge, H. T., Frimer, M., Stewart, D. K. \& Caldwell, J. Quantitative measurement of left ventricular volumes in man from radiopaque epicardial markers. Circulation 54, 391-398 (1976).

25. Gibson, D. G. Measurement of left ventricular volumes in man by echocardiography--comparison with biplane angiographs. Br Heart J 33, 614 (1971). 
Chapter 5, Part 3

The hindlimb: neither NOX4, nor NOX1, 2 or 5 contribute to chronic ischemia in the femoral artery ligation model 


\begin{abstract}
Peripheral artery disease is a chronic ischemic condition severely affecting quality of life of patients suffering from it and increasing their risk of other cardiovascular events. Chronic ischemia leads to a pro-angiogenic environment, allowing the tissue to restore the nutrient and oxygen delivery. NADPH oxidases are upregulated via HIF-1 $\alpha$ in ischemic conditions. They also can increase angiogenesis by facilitating the proliferation and differentiation of both endothelial and smooth muscle cells. The roles of the different NOX isoforms in angiogenesis caused by chronic ischemia are not fully elucidated yet, with current data being conflicting.

In this study, using the mouse model of femoral artery ligation, none of the vascular NADPH oxidases seems to play a role in blood flow recovery or angiogenesis in chronic ischemia. NOX4 has a small detrimental role at the short term, but long-term outcome is not affected. These results are conflicting with earlier results showing a beneficial role of NOX2 and NOX4 in angiogenesis. However, differences in methods might account for this discrepancy, since the amount of ischemia and possible interfering inflammation can affect the long-term angiogenesis. Taken together, the data show that NADPH oxidases are no major therapeutic targets in peripheral artery disease. In addition, it is clear that timing, amount and (sub)cellular localisation of ROS formation are important determinants of the final effects of oxidative stress.
\end{abstract}




\section{Introduction}

Peripheral artery disease (PAD) affects up to $30 \%$ of the worldwide population. In the decade from 2000 to 2010, the number of patients increased by $23,5 \%$ to about 200 million ${ }^{1}$. PAD is characterised by a stenosis of the arterial vessels in the legs, leading to walking induced pain and walking impairment, affecting almost all activities in daily life. In addition, patients with PAD (even the asymptomatic ones) are at higher risk of other cardiovascular diseases such as ischemic heart disease and stroke ${ }^{2}$. Atherosclerosis is the common underlying disease mechanism in these three diseases.

Atherosclerosis in the leg vessels leads to impairment in blood flow and thus a state of chronic ischemia in PAD. One of the cornerstones of treatment, next to decreasing risk factors, is to increase blood flow to the legs by surgically preparing bypasses or by stimulating the formation of collateral pathways by walking therapy ${ }^{3}$. Enhancing the formation of new blood vessels in PAD can increase quality of life for PAD patients; however, the precise underlying mechanisms of collateral growth and formation of new collateral vessels are incompletely understood.

In chronic ischemia, the process of angiogenesis is activated by oxygen depletion as a natural reparative mechanism ${ }^{4}$. Reactive oxygen species play a role here, in activating endothelial ${ }^{5,6}$ and smooth muscle cell proliferation ${ }^{7,8}$. NADPH oxidases, with their sole function of producing ROS have firstly been implicated in angiogenesis using in vitro models ${ }^{5,9-12}$. Also NADPH oxidases are involved in the hypoxiainducible-factor (HIF) pathway during ischemic conditions ${ }^{13-15}$. Activated HIF promotes angiogenesis via VEGF and other growth factors ${ }^{16}$.

The mouse model of femoral artery ligation has been used to study the role of NOX2 in chronic ischemia and remodelling with conflicting results ${ }^{17-21}$. NOX4 has been shown to be pro-angiogenic in vitro ${ }^{10,22}$ and in vivo ${ }^{22,23}$.

In this chapter, we for the first time address the role of all 4 vascular NADPH oxidase isoforms NOX1, 2, 4 and 5 in angiogenesis after chronic ischemia, using the in vivo mouse model of 3-vessel femoral artery ligation. 


\section{Results}

NOX4Ma and NOX4Wü mice show comparable results in the hindlimb ischemia model

To investigate if the Maastricht and Würzburg mice would have a different phenotype also in the hindlimb chronic ischemia model, NOX4 KO and WT mice from both colonies were investigated. As can be seen in Fig. 1, the blood flow restoration as measured by laser Doppler, did not differ between the mice from Maastricht and Wurzburg. Therefore, in the rest of this chapter, the data of these two NOX4 mouse groups have been combined.
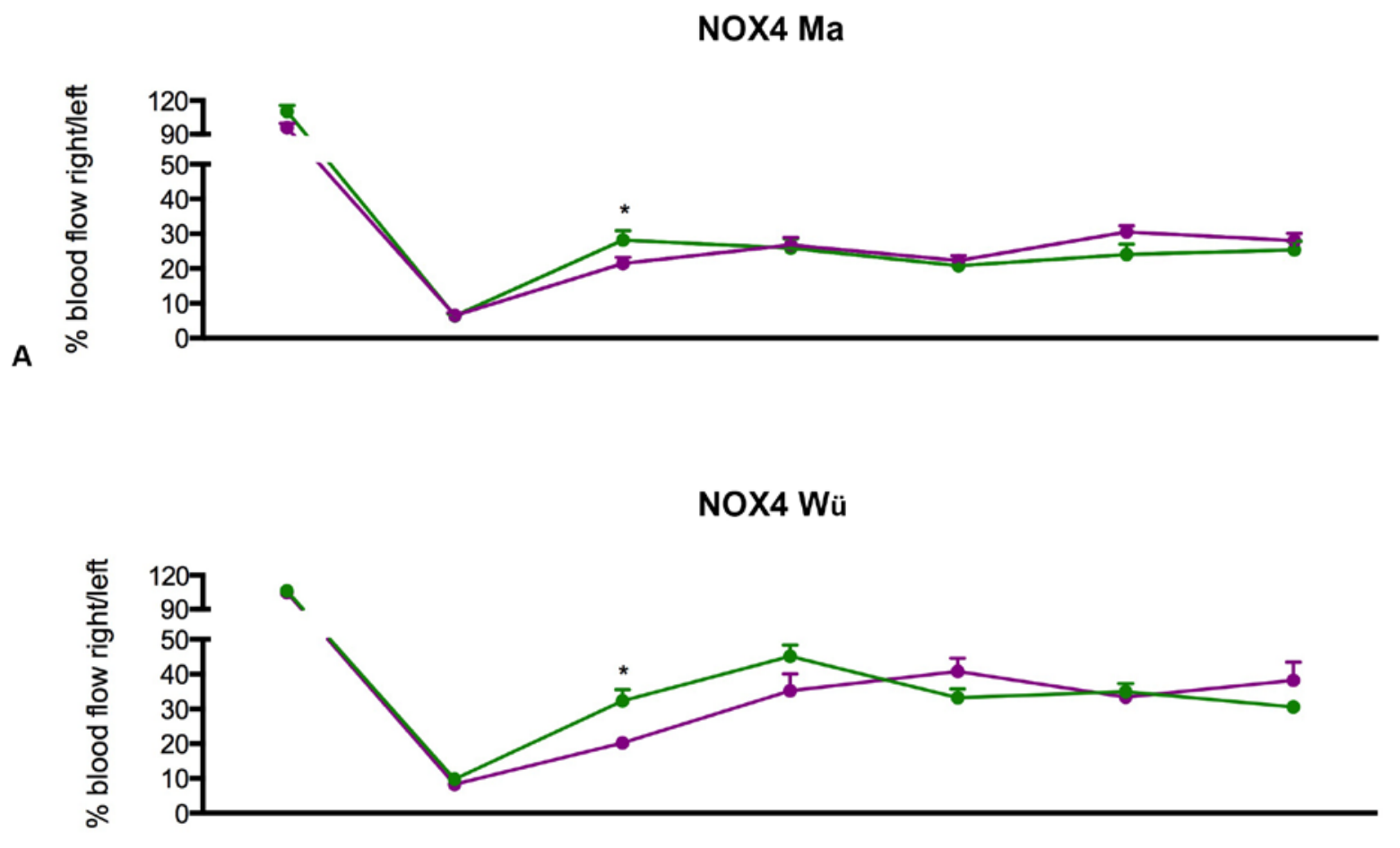

B

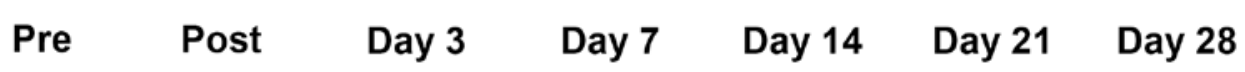

Figure 1. Blood flow restoration over time as measured by laser Doppler did not show any differences between the NOX4Ma and NOX4Wü mice. A) Maastricht NOX4 KO (purple, $n=12$ ) and $W T$ (green, $n=11$ ) mice show comparable blood flow restoration. At day 3 , the KO shows a lower recovery. B) Würzburg NOX4 KO (purple, $n=5$ ) and WT (green, $n=12$ ) mice show comparable blood flow restoration. At day 3 , the $K O$ shows a lower recovery. Data are presented as percentage blood flow of the ischemic paw over the non-ischemic paw (mean \pm SEM). * $p<0.05 \mathrm{KO}$ versus $W T$. 


\section{Blood flow restoration after ligation of the femoral artery is not affected by}

NOX1, 2, 4 and 5

Mice lacking NADPH oxidase 1, 2 or 4 and their respective matched WT's as well as mice expressing the human NOX5 gene (NOX5 KI) and their matched WT's, were subjected to ligation of the femoral artery to mimic peripheral artery disease to study angiogenesis. Baseline characteristics were similar comparing the different groups to their matched WT's (Table 1).

Table 1. Baseline characteristics of the mice subjected to hindlimb ischemia.

\begin{tabular}{lccc}
\hline & Age (weeks) & Weight (gram) & N= \\
\hline NOX1 WT & $10 \pm 0.5$ & $19 \pm 0.4$ & 14 \\
NOX1 KO & $10 \pm 0.4$ & $20 \pm 0.9$ & 11 \\
\hline NOX2 WT & $15 \pm 0.4$ & $23 \pm 1.1$ & 11 \\
NOX2 KO & $14 \pm 0.5$ & $25 \pm 0.9$ & 13 \\
\hline NOX4 WT & $13 \pm 0.5$ & $23 \pm 0.8$ & 17 \\
NOX4 KO & $12 \pm 0.5$ & $24 \pm 0.7$ & 23 \\
\hline NOX5 WT & $13 \pm 0.4$ & $27 \pm 1.4$ & 13 \\
NOX5 KI & $12 \pm 0.3$ & $29 \pm 1.6$ & 13 \\
\hline
\end{tabular}

Laser Doppler was used to follow up the amount of blood flow restoration over time. All groups of mice showed a significant drop in blood flow after surgery (Fig. 2), which then gradually increased again over 4 weeks to reach a maximum of $30,9 \pm 3,3 \%$ and $27,2 \pm 3,7 \%$ in the NOX1 KO and WT; $33,5 \pm 3,6 \%$ and $27,3 \pm 2,6 \%$ for the NOX2 KO and WT; $31,8 \pm 2,5 \%$ and $28,0 \pm 1,6 \%$ for the NOX4 KO and WT; and $42,3 \pm 3,6 \%$ and $42,5 \pm 2,2 \%$ for the NOX5 KI and WT mice respectively, compared to baseline. There were no significant differences in blood flow between any of the KO or KI groups compared to their matched WT at the long term (Fig. 2) In the NOX4 KO mice, recovery was impaired only at day 3 after ligation $(21,0 \pm 1,2 \%, n=17)$ compared to the WT $(30,3 \pm 2,1 \%, n=23)$. At day 7 after ligation, a small difference was still

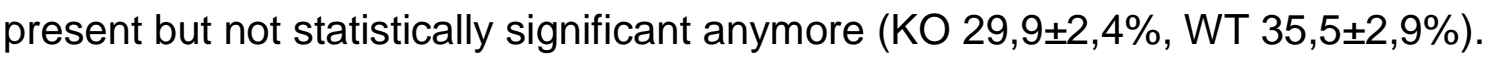

From day 14 onwards, the blood flow in the WT decreased again a little and stayed stable in the KO mice, abolishing the difference between the 2 genotypes at the end measurement. 
NOX1, 2, 4 and 5 do not affect capillary density after 4 weeks of chronic ischemia

As a measure of angiogenesis, capillary density was measured in the thigh and calf muscles using a CD31 immunohistochemical staining (Fig. 3).

NOX1 (Fig. 3A and B) KO mice showed a similar amount of capillaries in the gastrocnemius muscle $(927 \pm 111 \mathrm{cap} / \mathrm{mm}, \mathrm{n}=10)$ compared to the matched WT mice (880 $\pm 88 \mathrm{cap} / \mathrm{mm}, \mathrm{n}=13)$. The amount of capillaries in the adductor thigh muscle was lower than in the gastrocnemius muscle as expected, but again no differences between the 2 genotypes was observed (KO 559 \pm 59 cap/mm, n=11; WT $520 \pm 69 \mathrm{cap} / \mathrm{mm}, \mathrm{n}=12)$.

In the NOX2 (Fig. 3C and D) KO mice a small increase in capillary density was found (812 \pm 12 cap/mm, $n=12)$ compared to the WT $(664 \pm 87 \mathrm{cap} / \mathrm{mm}, \mathrm{n}=11)$ but this difference did not reach significance. Again, the adductor muscle showed less capillary formation than the gastrocnemius muscle with no differences between the genotypes (KO 369 $\pm 37 \mathrm{cap} / \mathrm{mm}, \mathrm{n}=12$; WT 322 $26 \mathrm{cap} / \mathrm{mm}, \mathrm{n}=10$ ).

NOX4 KO mice showed the same amount of capillaries in the gastrocnemius (653 $\pm 61 \mathrm{cap} / \mathrm{mm}, \mathrm{n}=15)$ and adductor (411 $\pm 52 \mathrm{cap} / \mathrm{mm}, \mathrm{n}=15)$ muscle when compared to the WT mice $(700 \pm 73 \mathrm{cap} / \mathrm{mm}, \mathrm{n}=22$ and $359 \pm 31 \mathrm{cap} / \mathrm{mm}, \mathrm{n}=19$, respectively).

The NOX5 (Fig. 3G and H) KI $(660 \pm 89 \mathrm{cap} / \mathrm{mm}, \mathrm{n}=14)$ and the NOX5 WT mice (583 $\pm 110 \mathrm{cap} / \mathrm{mm}, \mathrm{n}=13)$ also showed comparable capillary density in the gastrocnemius muscle. The adductor muscle capillary density did not differ between NOX5 KI (373 $\pm 43, \mathrm{n}=14)$ and NOX5 WT $(404 \pm 47, \mathrm{n}=13)$ mice.

In conclusion, none of the NADPH oxidases affected the amount of capillary formation in the thigh and calf muscles of mice subjected to femoral artery ligation. 


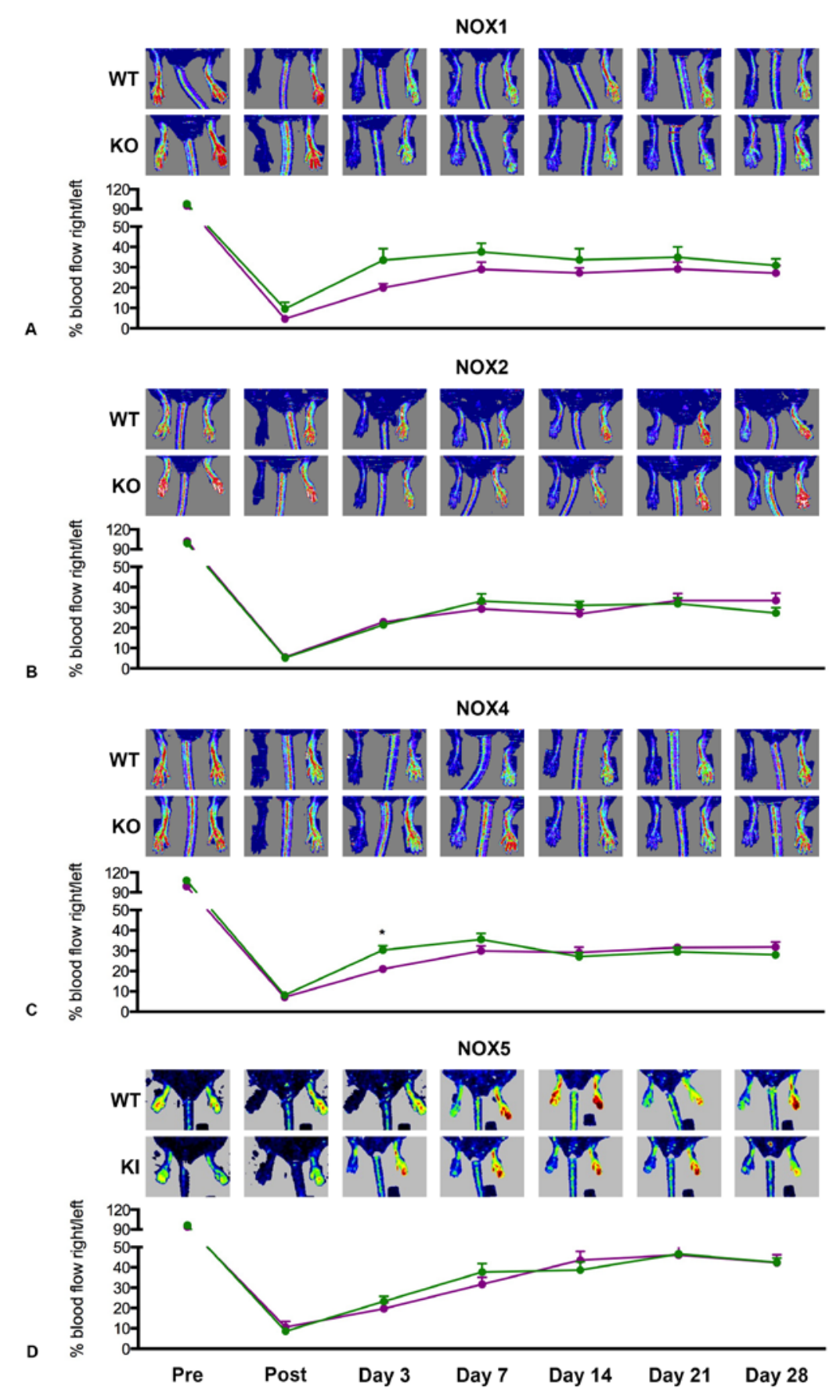

Figure 2. Blood flow restoration as measured by laser Doppler did not show any differences between $\mathrm{KO} / \mathrm{KI}$ and their respective WT mice. Data are presented as percentage blood flow

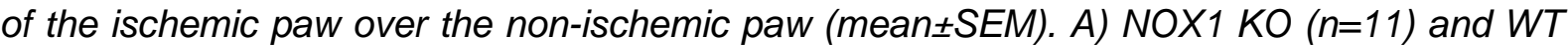
$(n=14)$ mice. B) NOX2 KO (n=11) and WT (n=12) mice. C) NOX4 KO (n=17) and WT $(n=23)$ mice. D) NOX5 KI (n=13) and WT $(n=13)$ mice. Due to technical problems with the Moor LDI Doppler, the NOX5 mice have been scanned using a Perimed laser Doppler system. Green lines represent $W T$, purple lines represent $K O / K I$ mice. 


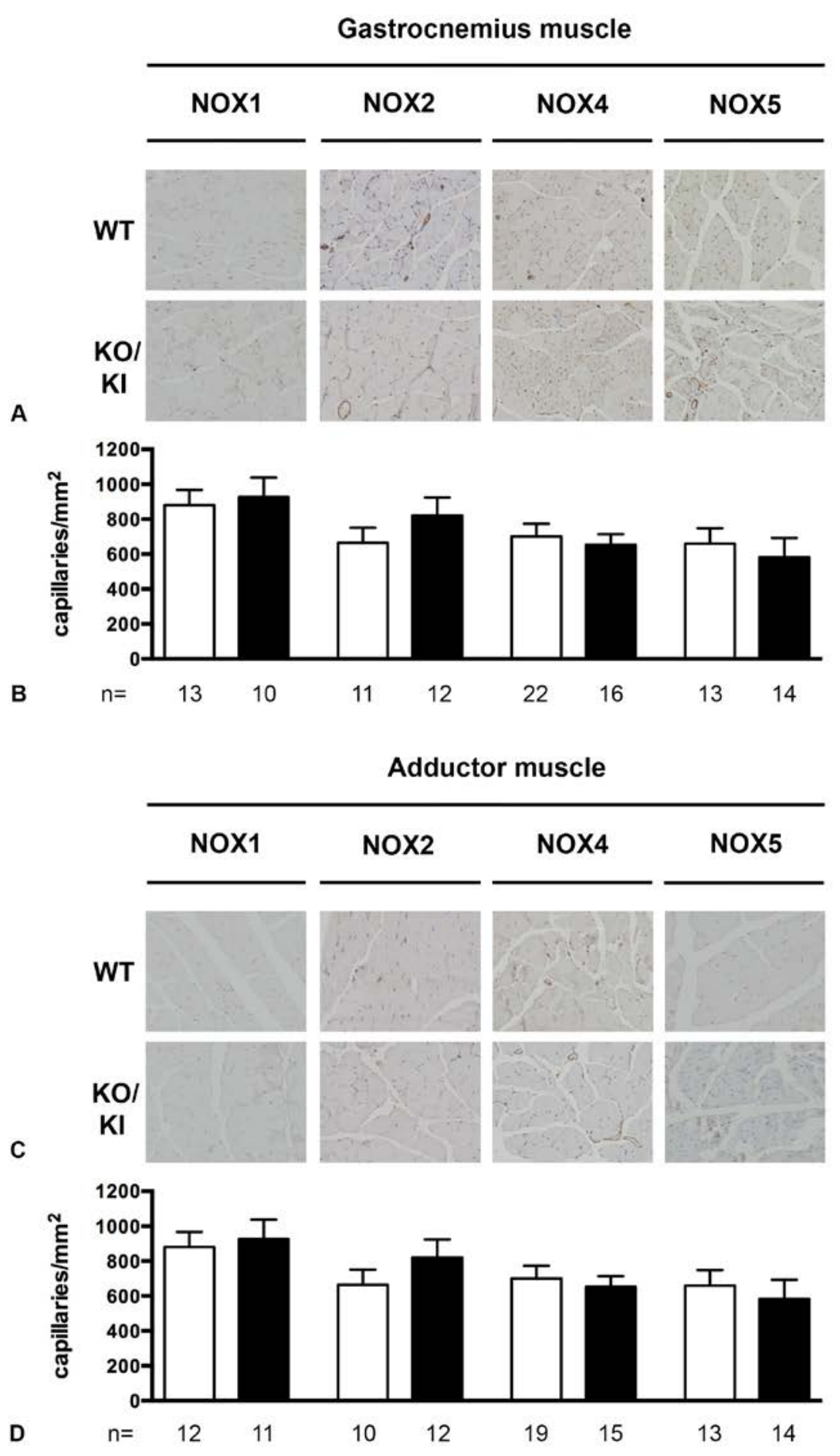

Figure 3. Capillary density as assessed by CD31 immunohistochemical staining did not show any differences between $\mathrm{KO} / \mathrm{KI}$ and their respective WT mice. A) Representative pictures of the CD31 stain in ischemic gastrocnemius muscle. B) Quantified number of capillaries in the ischemic gastrocnemius muscle. C) Representative pictures of the CD31 stain in ischemic adductor muscle. D) Quantified number of capillaries in the ischemic adductor muscle Bars represent mean \pm SEM of $n$ experiments as mentioned below each bar. White bars represent WT, black bars represent KO/KI mice. 


\section{Oxidative stress is not affected by the NADPH oxidases}

Using dihydroethidium staining of cryosections, the total amount of ROS production in ischemic adductor (thigh) and gastrocnemius (calf) muscles was measured (Fig. 4). Fluorescence intensity in the NOX 1 KO calf $(100.8 \pm 8.8, n=10))$ and thigh $(67.8 \pm 4.5, n=10)$ muscle did not differ when compared to their respective WT (85.8 $\pm 8.8, n=12$ and $80.4 \pm 4.6, n=14$ respectively). The group of NOX2 mice showed values in the calf muscle that were in the same range, with no significant effect between genotypes ( $K O$ calf $74.9 \pm 4.6, n=12$, WT calf $86.0 \pm 17.7, n=11$ ). In the thigh muscles of NOX2 KO mice an increased fluorescence level was measured compared to the WT (KO 77.2 $\pm 6.2, n=12$; WT 67.6 $\pm 6.5, n=11$ ), but this did not reach statistical significance. NOX4 KO mice showed higher ROS levels, although not significant in the calf $(73.5 \pm 8.4, n=11)$ and similar levels in the thigh $(84.8 \pm 11.7, n=12)$ compared to their matched WT (calf $69.4 \pm 7.3, n=10$; thigh $100.2 \pm 15.5, n=10$ ). A trend towards more ROS in the thigh (KI 82.1 $\pm 11.8, n=14$, WT 76.3 $\pm 8.6, n=14)$ and calf muscles (KI 95.3 $\pm 11.3, n=14$; WT $72.8 \pm 8.8, n=13$ ), was seen in NOX5 KI mice compared to the WT, but this did not reach significance.

In conclusion, also the ROS production in the ischemic muscles was not affected by any of the NADPH oxidase isoforms.

\section{Discussion}

Our results suggest that NADPH oxidase 4 is not involved in blood flow restoration, angiogenesis or ROS production after chronic ischemia of the hindlimb. These results are conflicting with one other study, stating that NOX4 KO mice (both constitutive and tamoxifen-induced) show a decreased blood flow recovery and lower capillary density compared to the matched WT's. Injecting WT mice locally with adenoviral NOX4 and EC specific NOX4 overexpressing mice were found to have enhanced blood flow recovery and capillary density ${ }^{22}$. These data point towards a protective role of NOX4 in chronic ischemia and angiogenesis. 


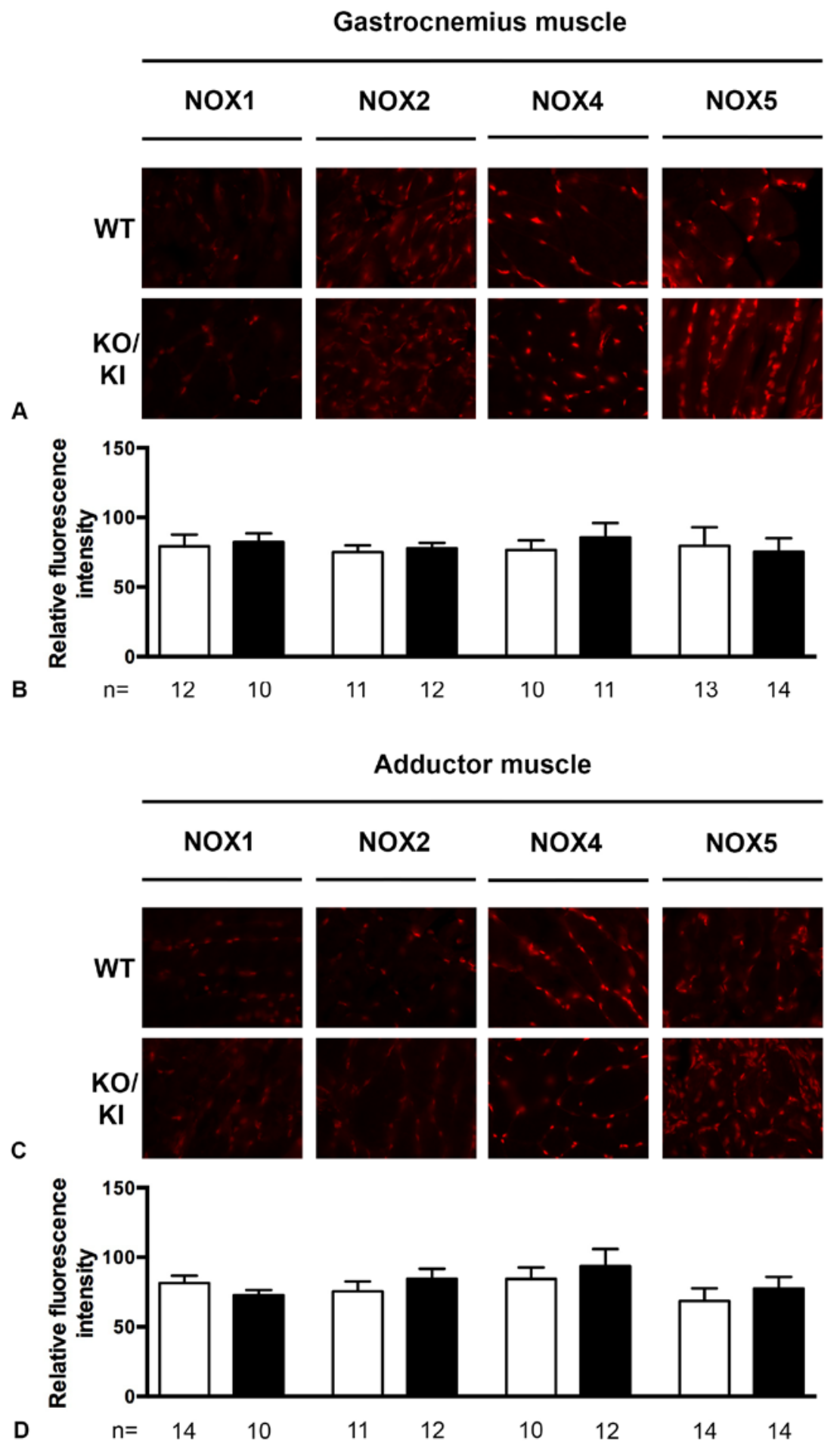

Figure 4. Oxidative stress as assessed by DHE staining did not differ between the KO/KI and their respective WT mice. A,C) Representative pictures of fluorescent DHE staining in the ischemic gastrocnemius ( $A$ ) and adductor muscles (C). $B, D$ ) Average intensity of nuclear staining in gastrocnemius (B) and adductor (D) muscles. Bars represent mean \pm SEM of $n$ experiments as mentioned below each bar. White bars represent WT, black bars represent KO/KI mice. 
However, as already discussed in the heart section of this chapter, different NOX4 $\mathrm{KO}$ strategies have been used and cell specific effects might influence the findings as well. Over the course of 4 weeks, the Doppler flow measurements of blood flow showed a rather low recovery rate when compared to other studies using NOX KO mice ${ }^{20,21,23,24}$, although others have also observed recoveries as low as ours ${ }^{25-27}$. Looking at the 'clinical' picture, our mice did not show any signs of necrosis and after approximately one week, the hindlimb was used in an apparently fully normal manner. In a pilot to our experiments, we attempted to include paw functionality measurements into our experimental protocol by using the CATWALK, a routine motility outcome in different neurological models ${ }^{28,29}$. Mice walk over a glass plate with a fluorescent light in it; a camera underneath can detect the paw prints that appear when the animal walks. These data can then be used to analyse the gait in a quantitative manner. In the first week of our ischemia model, the mice did not use their ligated paw at all, making it impossible to measure time the mice stand on their paw while walking, or the pressure they put on it. From the second week onwards, the mice rapidly started to fully use their paws again, making it impossible to detect any subtle differences between KO and WT mice. Whilst these observations showed that the functionality fully recovers in our model, the CATWALK could not be used, neither in the first nor in the second week. The discrepancy between the substantial functional recovery and the low blood flow restoration may indicate a lack of correlation between either blood flow and function, or between Doppler signal and true blood flow in the paws. It is also known that Doppler measurements are sensitive to small changes in position of the subject, blood pressure, temperature and blood cell concentration, which may further complicate analysis ${ }^{30}$. One method to overcome the latter confounders is by using the non-ischemic paw of the same animal as internal control; however, variations between different measurement days and differences in the methods between labs (especially anaesthetics) are still a source of major discrepancies.

In addition, there is a large variation in mouse hindlimb ischemia methods, either non-invasive ${ }^{31,32}$ or invasive ${ }^{33-35}$. The non-invasive techniques, using tourniquets, are real ischemia-reperfusion experiments and have been criticised because pressure of the tourniquet also damages the muscles and nerves itself ${ }^{31}$. 
Excision of the femoral artery is one method that results in much more damage to surrounding tissues than just ligating the femoral artery ${ }^{33}$. In addition, variations in the way of interrupting blood flow (ligation vs electrocoagulation), the place of ligating and also occluding the vein or not can result in major changes in the response afterwards ${ }^{33,34,36}$. Different mouse strains also show different responses to hindlimb ischemia ${ }^{37,38}$ and even if perfusion is the same, occurrence of necrosis can differ between mouse strains ${ }^{36}$.

Both angiogenesis and arteriogenesis play a role in the processes in chronic ischemia, but to different degrees ${ }^{39,40}$. This could also explain discrepancies between different models. The major stimulus for angiogenesis is ischemia, with a resulting upregulation of VEFG, but arteriogenesis is mainly stimulated via alterations in shear stress ${ }^{41,42}$. The difference at day 3 post-ischemia we saw between the NOX4 WT and KO mice is probably related to an effect on angiogenesis, since this process occurs first ${ }^{43}$ and NOX4 has also been implicated in angiogenesis ${ }^{10,44}$. Within our model, the long-term outcome was not affected, excluding NOX4 as a major target in PAD.

After excluding NOX4 as a therapeutic target, we could also show that neither NOX1 nor NOX2 play a role in the same ischemic hindlimb model. NOX1 has earlier been described to have a detrimental role in hindlimb ischemia, with NOX1 KO mice showing a better blood flow recovery ${ }^{23}$.

NOX2 has been extensively studied before in the hindlimb ischemia model. Four papers describe a beneficial role of NOX2 in the angiogenic response to hindlimb ischemia, with NOX2 KO mice having reduced or delayed blood flow recovery and impaired neovascularisation ${ }^{18-21}$. One of these studies also tested the same hindlimb model in streptozotocin-diabetic mice; here a detrimental role of NOX2 was found with a restoration of the impaired neovascularisation in NOX2 KO diabetic mice ${ }^{19}$. Also in conditions of oxidative stress NOX2 is detrimental in the recovery after hindlimb ischemia, as was shown in a model of chronic smoke exposure ${ }^{17}$. In this same study, also under basal conditions, a detrimental role of NOX2 in hindlimb ischemia was found. These results seem contrasting to the other studies and our own data in addition show no role of NOX2. 
In humans, one study in chronic granulomatous disease patients (which have a NOX2 deficiency) showed a preserved endothelial function after ischemiareperfusion of the arm, while normal controls showed endothelial dysfunction with lower flow-mediated dilation ${ }^{45}$. This would also point towards a detrimental role of NOX2 in ischemia-reperfusion. When taking all these studies together, we can conclude that the effect of NOX2 on PAD would be only minor if an effect would be present at all.

We also tested the role of the fourth vascular NOX isoform, NOX5 in chronic hindlimb ischemia, using our humanised eNOX5 KI mouse. Again, no differences between WT and eNOX5 KI mice could be found in any of the parameters measured, excluding also NOX5 as a target in PAD.

One of the major limitations of all hindlimb ischemic studies and thus also our own study, is that the mouse hindlimb ischemic models do not represent accurately the human peripheral artery disease. In humans, there is mostly a gradual decrease in blood flow with increasing ischemia, leading to a gradual formation of collateral pathways. In the mouse models of hindlimb ischemia, a sudden total blood flow stop occurs that is more artificially and maybe affects shear stress more, leading to more arteriogenic processes. The model we used is a rather mild ischemic model, in which almost no necrosis occurs. Thus, this mimics better the PAD condition in humans when compared to severe ischemic models. However, although the mild ischemia resembles more closely the clinical situation in patients, with less ischemia, also the angiogenic response will be less (since hypoxia is the main trigger). This makes it more difficult to detect small differences in blood flow restoration. One may question whether these very small differences would be relevant in a clinical setting at all. In conclusion, our results show that vascular NADPH oxidases do not play a role in angiogenesis after chronic hindlimb ischemia and are thus not major therapeutic targets for peripheral artery disease. The conflicting results within the hindlimb studies but also between the hindlimb and stroke studies again point towards amount-, timing- and localisation specific effects of ROS in ischemia-reperfusion injury. 


\section{Methods}

\section{Animals}

All animal experiments were approved by the institutional animal experimental committee and performed according to the Dutch law on animal experiments. Animals were housed under controlled conditions $\left(22^{\circ} \mathrm{C}, 55-65 \%\right.$ humidity, $12 \mathrm{~h}$ lightdark cycle), and were allowed free access to water and standard laboratory chow. Male and female animals aged 12-16 weeks were used. The NOX1 KO, NOX2 KO, NOX4 KO and NOX5 KI animals were compared to their respective matched WT's.

\section{Doppler measurements}

After administration of a painkiller (buprenorphine s.c $0.05 \mathrm{mg} / \mathrm{kg}$ ), the mouse was anesthetised with isoflurane $4-5 \%$ and both groins were shaved. The mouse was then placed on the heating plate of the Moor Laser-Doppler, with its paws taped to the underground with double sided tape. Anaesthesia was then maintained with $1,5-2 \%$ isoflurane in air via a vaporizor (Univentor, UNO Roestvaststaal BV) connected to a ventilation mask. The mouse was allowed to heat up for 10 minutes before starting the scan.

Three consecutive scans were made for each mouse. An area of interest was drawn around the paws and the mean color pixel value was calculated per paw and expressed as ratio of the ligated over the non-ligated leg.

\section{Ligation femoral artery}

Directly after the basal Doppler measurement, anaesthesia was maintained with 1,5-2\% isoflurane in air via a vaporizor (Univentor, UNO Roestvaststaal BV) connected to a ventilation mask.

The mouse was placed on the back on a heating pad (UNO temperature control unit, UNO Roestvaststaal BV) and body temperature was monitored using a rectal probe and maintained at $37.0^{\circ} \mathrm{C}$ using a feedback-controlled infrared light. The right groin was disinfected and via a small incision, the femoral artery was dissected free from the femoral vein and nerve. A suture (5-0 silk) was placed around the femoral artery in between the branching of the a. epigastrica and the a. poplitea. 
These last two arteries were also dissected free from their veins and nerves and ligated to prevent collateral flow and backflow respectively (Fig. 5). The wound was then closed with a 4-0 polysorb suture and the mouse was allowed to recover. Discoloration of the paw was visible after the ligating surgery (Fig. 6) and an accompanying drop in blood flow was visible with laser Doppler.

\section{Tissue sampling}

After the last Doppler measurement at day 28, animals were kept under isoflurane anaesthesia and the musculus adductor and musculus gastrocnemius were prepared free, dissected and divided into 2 pieces for formalin fixation and embedding in OCT respectively.

\section{Capillary density}

Formalin-fixed, paraffin embedded sections of $4 \mu \mathrm{m}$ were deparaffinised and quickly rehydrated. Endogenous peroxidase activity was quenched by incubation in 0,3\% $\mathrm{H}_{2} \mathrm{O}_{2}$ in PBS for 30 minutes at room temperature. Antigen retrieval was achieved by heat induced epitope retrieval using a 0.01M citrate buffer $(\mathrm{pH} 6.0)$. Non-specificbinding was blocked by incubating the slides in 5\% normal swine serum in PBS for 30 minutes. Thereafter, slides were incubated at $4^{\circ} \mathrm{C}$ overnight with the primary antibody, monoclonal rat anti-mouse antibody to CD31 (PECAM-1) (HistonovaDianova, Cat. no DIA310) diluted 1:50. As secondary antibody, biotin labelled rabbit anti-rat antibody (dakocytomotion Denmark no. E0468) was used diluted 1:200 (incubation for 30 minutes), followed by the $A B C$-vectastain kit and 3,3diaminobenzidine. Counterstaining with Mayer's haematoxylin was used.

Pictures were taken using a Leica camera connected to a Zeiss microscope. Pictures were analysed using the Leica Qwin pro v3.5.1 software. For each animal, three random pictures were taken per muscle sample and the amount of capillaries is expressed as number per square $\mathrm{mm}$. 


\section{Oxidative stress}

The amount of general ROS production was measured using dihydroethidium (DHE, sigma Aldrich, D7008, lot no SLBD6013V) dissolved in Krebs-HEPES buffer ( $\mathrm{NaCl}$ 99mM, KCl 4.7mM, $\mathrm{MgSO}_{4} 2.5 \mathrm{mM}, \mathrm{CaCl}_{2} 2.5 \mathrm{mM}, \mathrm{K}_{2} \mathrm{HPO}_{4} 1 \mathrm{mM}$, Na-HEPES $20 \mathrm{mM}$, glucose $5.6 \mathrm{mM}$ ). Cryosections of $7 \mu \mathrm{m}$ were incubated in $2 \mu \mathrm{M}$ DHE for 5 minutes at room temperature. Fluorescence intensity was then measured using a Zeiss filter (excitation 510nm, emission 590nm) and a fluorescent light. Three random pictures of each sample were taken using a Leica camera connected to a Zeiss microscope. Mean fluorescence intensity of the nuclei was measured using the Leica Qwin pro v3.5.1 software.

\section{Statistics}

All data are expressed as mean \pm SEM. Using the GraphPad Prism 5.0 software package statistical differences between mean values were determined by Student's two-tailed $t$ test. For repeated measurements (Doppler), a two-way ANOVA was used. A value of $p<0.05$ was considered to be statistically significant.

\section{Power}

To ensure sufficient statistical power $(>80 \%)$ for our experiments, the number of animals needed per group was calculated for the most relevant, i.e. primary outcome, blood flow restoration. Based on an alpha of 0.05 , a detectable difference of at least $25 \%$ and a standard deviation of $15 \%$ (based on literature and previous experience within the university with this model), $n=6$ animals per group were needed to ensure a power of $80 \%$ according to Sachs' formula (Sachs, Angewandte Statistik). Post hoc analysis showed a larger variation than expected, but all groups had a power around 80\% (Table 2). 
Table 2. Power analysis Doppler measurements.

\begin{tabular}{|c|c|c|c|c|c|c|c|c|c|}
\hline \multirow[t]{3}{*}{ Isoform } & \multicolumn{8}{|c|}{ Blood flow restoration day 28} & \multirow{3}{*}{$\begin{array}{c}\text { Power for } \\
40 \% \\
\text { difference }\end{array}$} \\
\hline & \multicolumn{4}{|c|}{ WT } & \multicolumn{4}{|c|}{$\mathrm{KO} / \mathrm{KI}$} & \\
\hline & $\mathrm{N}$ & $\begin{array}{l}\text { Mean (\% } \\
\text { right/left) }\end{array}$ & SD & $\mathrm{CV}$ & $\mathrm{N}$ & $\begin{array}{l}\text { Mean (\% } \\
\text { right/left) }\end{array}$ & SD & CV & \\
\hline NOX1 & 14 & 30,9 & 12,2 & 0,39 & 11 & 27,2 & 12,2 & 0,45 & 79,9 \\
\hline NOX2 & 11 & 27,3 & 8,6 & 0,32 & 13 & 33,5 & 13,0 & 0,39 & 78,4 \\
\hline NOX4 & 22 & 28,0 & 7,6 & 0,27 & 19 & 31,8 & 11,1 & 0,35 & 95,6 \\
\hline NOX5 & 13 & 42,5 & 8,0 & 0,19 & 13 & 42,3 & 14,1 & 0,33 & 82,1 \\
\hline
\end{tabular}

To assess the power of our studies, the pooled variance of all groups was used to calculate the power for detecting a 25\% difference using the Russ Lenth's power and sample size software (http://homepage.stat.uiowa.edu/ rlenth/Power/index.html). CV, coefficient of variations (=SD/mean); $S D$, standard deviation.

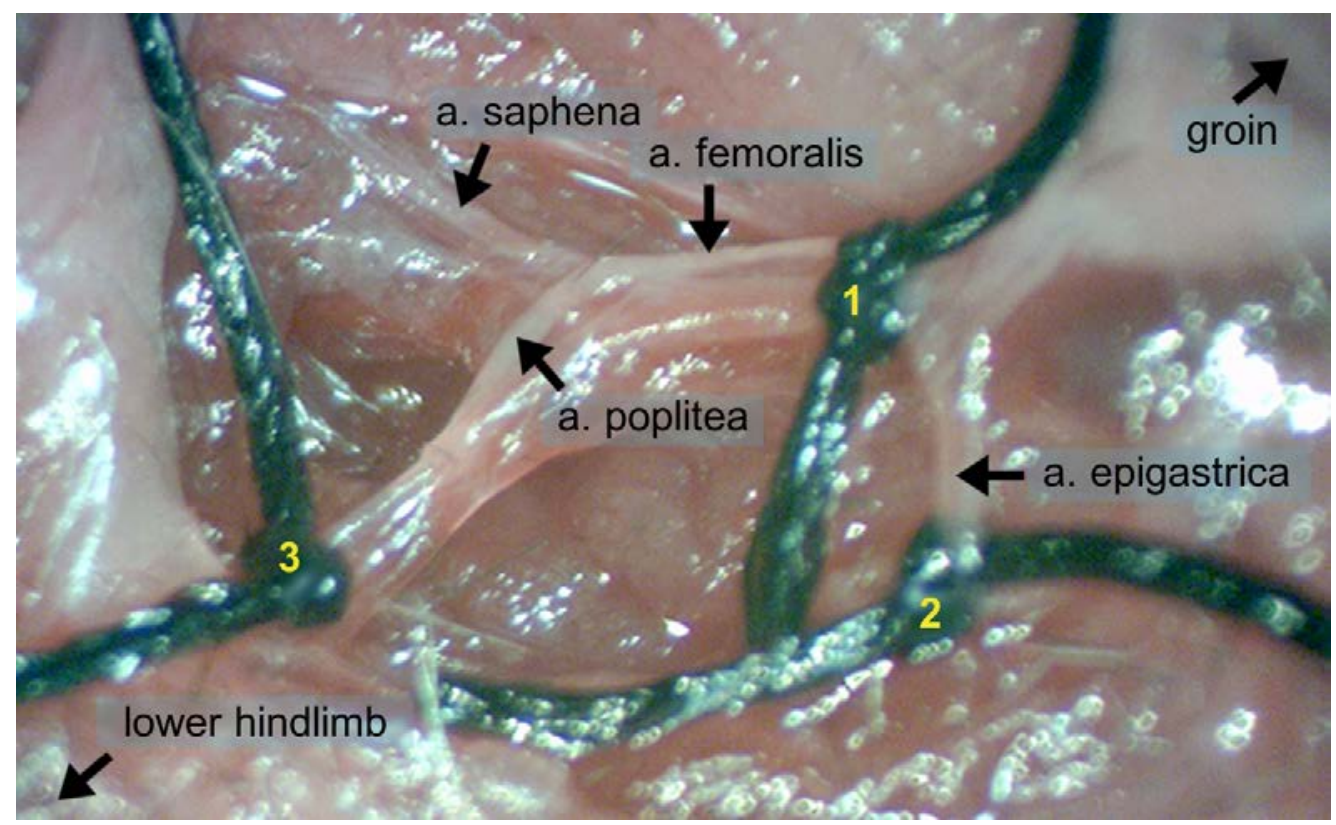

Figure 5. Surgical procedure for producing hindlimb ischemia. The femoral artery is ligated between the branching to the epigastric and popliteal arteries (1). The epigastric artery is ligated (2) to prevent collateral superficial flow, the popliteal artery is ligated (3) to prevent backflow. 

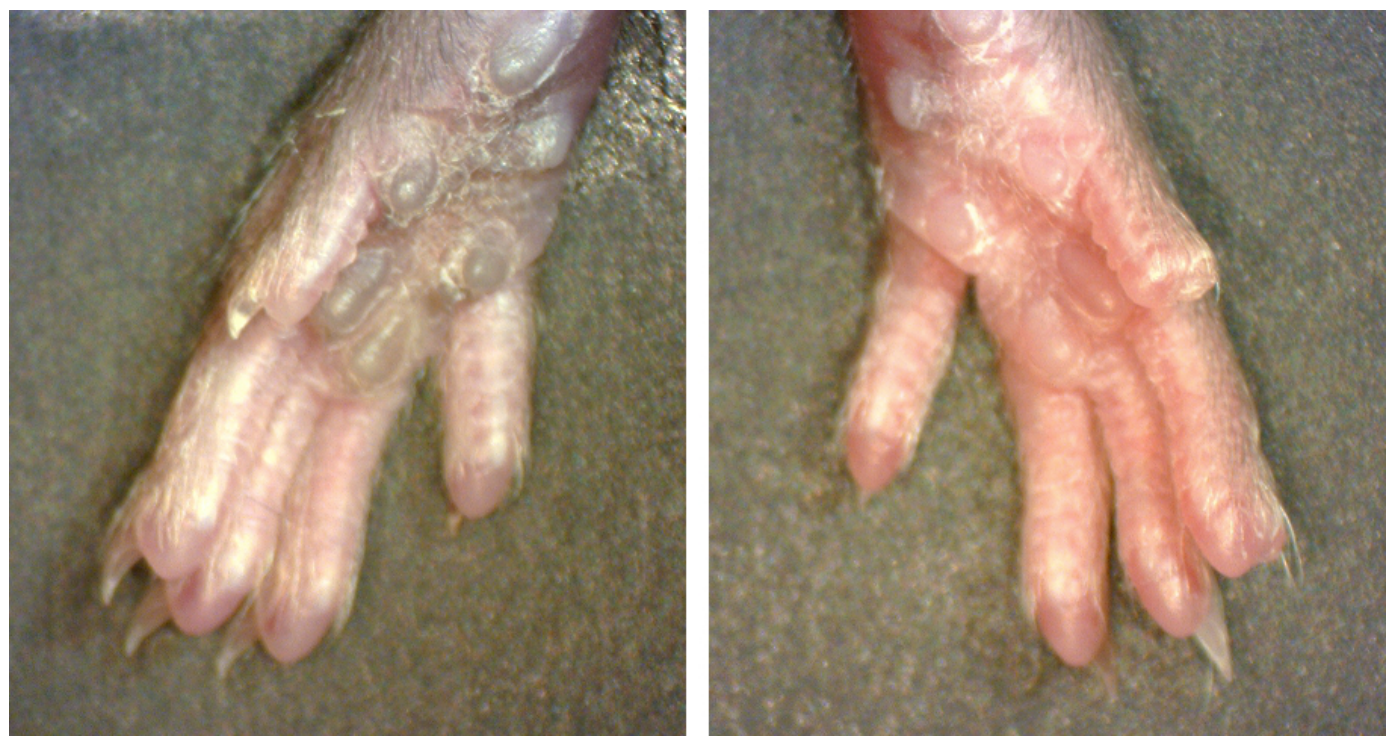

Figure 6. Visual assessment of the drop in blood flow. The ligated right paw (left) shows a greyish colour compared to the pink-red colour of the non-ligated left paw (right).

\section{References}

1. Fowkes, F. G. R. et al. Comparison of global estimates of prevalence and risk factors for peripheral artery disease in 2000 and 2010: a systematic review and analysis. Lancet 382, 1329-1340 (2013).

2. Diehm, C. Mortality and vascular morbidity in older adults with asymptomatic versus symptomatic peripheral artery disease. Circulation 120, 2053-2061 (2009).

3. Anderson, J. L. Management of patients with peripheral artery disease (compilation of 2005 and 2011 ACCF/AHA guideline recommendations): a report of the American College of Cardiology Foundation/American Heart Association Task Force on Practice Guidelines. Circulation 127, 1425-1443 (2013).

4. Maulik, N. \& Das, D. K. Redox signaling in vascular angiogenesis. Free Radic Biol Med 33, 1047-1060 (2002).

5. Abid, M. R., Kachra, Z., Spokes, K. C. \& Aird, W. C. NADPH oxidase activity is required for endothelial cell proliferation and migration. FEBS Lett. 486, 252256 (2000). 
6. Schäfer, M., Schäfer, C., Ewald, N., Piper, H. M. \& Noll, T. Role of redox signaling in the autonomous proliferative response of endothelial cells to hypoxia. Circ Res 92, 1010-1015 (2003).

7. Lyle, A. N. \& Griendling, K. K. Modulation of vascular smooth muscle signaling by reactive oxygen species. Physiology (Bethesda) 21, 269-280 (2006).

8. Papaharalambus, C. A. \& Griendling, K. K. Basic mechanisms of oxidative stress and reactive oxygen species in cardiovascular injury. Trends Cardiovasc. Med. 17, 48-54 (2007).

9. Arbiser, J. L. et al. Reactive oxygen generated by Nox1 triggers the angiogenic switch. Proc Natl Acad Sci U S A 99, 715-720 (2002).

10. Datla, S. R. et al. Important role of Nox4 type NADPH oxidase in angiogenic responses in human microvascular endothelial cells in vitro. Arterioscler Thromb Vasc Biol 27, 2319-2324 (2007).

11. Ushio-Fukai, M. et al. Novel role of gp91(phox)-containing NAD(P)H oxidase in vascular endothelial growth factor-induced signaling and angiogenesis. Circ Res 91, 1160-1167 (2002).

12. Meng, D. et al. NADPH Oxidase 4 Mediates Insulin-Stimulated HIF-1 $\alpha$ and VEGF Expression, and Angiogenesis In Vitro. PLOS ONE 7, e48393 (2012).

13. Diebold, I., Petry, A., Hess, J. \& Gorlach, A. The NADPH oxidase subunit NOX4 is a new target gene of the hypoxia-inducible factor-1. Mol Biol Cell 21, 2087-2096 (2010).

14. Diebold I, P. A. S. K. D. T. H. J. G. The HIF1 target gene NOX2 promotes angiogenesis through urotensin-II. J Cell Sci In press,

15. Block, K. et al. NAD(P)H oxidases regulate HIF-2alpha protein expression. $J$ Biol Chem 282, 8019-8026 (2007).

16. Pugh, C. W. \& Ratcliffe, P. J. Regulation of angiogenesis by hypoxia: role of the HIF system. Nat. Med. 9, 677-684 (2003).

17. Haddad, P. et al. Nox2-containing NADPH oxidase deficiency confers protection from hindlimb ischemia in conditions of increased oxidative stress. Arterioscler Thromb Vasc Biol 29, 1522-1528 (2009).

18. Distasi, M. R. et al. Suppressed hindlimb perfusion in Rac2-/- and Nox2-/- mice does not result from impaired collateral growth. Am. J. Physiol. Heart Circ.

Physiol. 296, H877-86 (2009). 
19. Ebrahimian, T. G. et al. NADPH oxidase-derived overproduction of reactive oxygen species impairs postischemic neovascularization in mice with type 1 diabetes. Am. J. Pathol. 169, 719-728 (2006).

20. Tojo, T. et al. Role of gp91phox (Nox2)-containing $\mathrm{NAD}(\mathrm{P}) \mathrm{H}$ oxidase in angiogenesis in response to hindlimb ischemia. Circulation 111, 2347-2355 (2005).

21. Urao, N. et al. Role of nox2-based NADPH oxidase in bone marrow and progenitor cell function involved in neovascularization induced by hindlimb ischemia. Circ Res 103, 212-220 (2008).

22. Craige, S. M. et al. NADPH oxidase 4 promotes endothelial angiogenesis through endothelial nitric oxide synthase activation. Circulation 124, 731-740 (2011).

23. Schroder, K. et al. Nox4 is a protective reactive oxygen species generating vascular NADPH oxidase. Circ Res 110, 1217-1225 (2012).

24. Haddad, J. J. \& Land, S. C. A non-hypoxic, ROS-sensitive pathway mediates TNF-alpha-dependent regulation of HIF-1alpha. FEBS Lett. 505, 269-274 (2001).

25. Oyama, O. et al. The lysophospholipid mediator sphingosine-1-phosphate promotes angiogenesis in vivo in ischaemic hindlimbs of mice. Cardiovasc Res 78, 301-307 (2008).

26. Kubo, M. et al. Hypoxic preconditioning increases survival and angiogenic potency of peripheral blood mononuclear cells via oxidative stress resistance. Am. J. Physiol. Heart Circ. Physiol. 294, H590-5 (2008).

27. Nakagami, H. et al. Novel autologous cell therapy in ischemic limb disease through growth factor secretion by cultured adipose tissue-derived stromal cells. Arterioscler Thromb Vasc Biol 25, 2542-2547 (2005).

28. Hamers, F. P., Lankhorst, A. J., van Laar, T. J., Veldhuis, W. B. \& Gispen, W. $\mathrm{H}$. Automated quantitative gait analysis during overground locomotion in the rat: its application to spinal cord contusion and transection injuries. J. Neurotrauma 18, 187-201 (2001).

29. Koopmans, G. C. et al. The assessment of locomotor function in spinal cord injured rats: the importance of objective analysis of coordination. $J$. Neurotrauma 22, 214-225 (2005). 
30. Leahy, M. J., de Mul, F. F., Nilsson, G. E. \& Maniewski, R. Principles and practice of the laser-Doppler perfusion technique. Technol Health Care 7, 143162 (1999).

31. Crawford, R. S. et al. A novel model of acute murine hindlimb ischemia. Am. J. Physiol. Heart Circ. Physiol. 292, H830-7 (2007).

32. Bonheur, J. A., Albadawi, H., Patton, G. M. \& Watkins, M. T. A noninvasive murine model of hind limb ischemia-reperfusion injury. J. Surg. Res. 116, 5563 (2004).

33. Goto, T. et al. Search for appropriate experimental methods to create stable hind-limb ischemia in mouse. Tokai J. Exp. Clin. Med. 31, 128-132 (2006).

34. Hellingman, A. A. et al. Variations in surgical procedures for hind limb ischaemia mouse models result in differences in collateral formation. Eur $\mathrm{J}$ Vasc Endovasc Surg 40, 796-803 (2010).

35. Couffinhal, T. et al. Mouse model of angiogenesis. Am. J. Pathol. 152, 16671679 (1998).

36. Shireman, P. K. \& Quinones, M. P. Differential necrosis despite similar perfusion in mouse strains after ischemia. J. Surg. Res. 129, 242-250 (2005).

37. Helisch, A. et al. Impact of mouse strain differences in innate hindlimb collateral vasculature. Arterioscler Thromb Vasc Biol 26, 520-526 (2006).

38. Chalothorn, D., Clayton, J. A., Zhang, H., Pomp, D. \& Faber, J. E. Collateral density, remodeling, and VEGF-A expression differ widely between mouse strains. Physiol. Genomics 30, 179-191 (2007).

39. Scholz, D. et al. Contribution of arteriogenesis and angiogenesis to postocclusive hindlimb perfusion in mice. J. Mol. Cell. Cardiol. 34, 775-787 (2002).

40. Ito, W. D. et al. Angiogenesis but not collateral growth is associated with ischemia after femoral artery occlusion. Am J Physiol 273, H1255-65 (1997).

41. Buschmann, I. \& Schaper, W. Arteriogenesis Versus Angiogenesis: Two Mechanisms of Vessel Growth. News Physiol. Sci. 14, 121-125 (1999).

42. Heil, M., Eitenmüller, I., Schmitz-Rixen, T. \& Schaper, W. Arteriogenesis versus angiogenesis: similarities and differences. J. Cell. Mol. Med. 10, 45-55 (2006). 
43. Hershey, J. C. et al. Revascularization in the rabbit hindlimb: dissociation between capillary sprouting and arteriogenesis. Cardiovasc Res 49, 618-625 (2001).

44. Peshavariya, H. et al. NADPH oxidase isoform selective regulation of endothelial cell proliferation and survival. Naunyn Schmiedebergs Arch. Pharmacol. 380, 193-204 (2009).

45. Loukogeorgakis, S. P. et al. Role of NADPH oxidase in endothelial ischemia/reperfusion injury in humans. Circulation 121, 2310-2316 (2010). 


\section{Chapter 6}

\section{Back to the brain: The role of NOX5 in stroke and hypertension}

To finalise our target validation on the possible role of NADPH oxidase in stroke, we finally investigated the role of NOX 5, the isoform that is not present in rodents but in humans and which has so far been understudied by many investigators. For this it was necessary to generate a 'humanised' mouse expressing the human NOX5 gene. In the course of this, we also identified a possible role for NOX5 in hypertension, which is likely to open up yet another chapter in NOXology. 


\begin{abstract}
After our journey from the brain to the periphery, investigating all vascular NOX isoforms in heart and hindlimb ischemia, we can conclude that so far the role of NOX4 in IRI appears to be specific to the brain, while NOX1 and 2 do not seem to play a role at all. The remaining vascular NOX isoform, NOX5 has been found to be upregulated in hypertension in humans. However, rodents lack this possible key human risk gene for stroke. Thus, in this chapter we investigated the role of this understudied NADPH oxidase isoform in stroke and hypertension, using mice expressing the human NOX5 gene in vascular endothelial cells. Systolic blood pressure and mean arterial pressure were increased in both genders of aged NOX5 KI mice. Surprisingly, only females showed also an increase in diastolic blood pressure. In addition, upon experimental ischemic stroke in young mice, NOX5 KI females had increased infarct sizes, worse neurological outcomes and more blood brain barrier leakage. Blood pressure in the young mice was not found to be elevated in pilot experiments, excluding a direct link between NOX5, hypertension and stroke. Rather, there seems to be a common underlying mechanism, which could be endothelial dysfunction caused by NOX5.
\end{abstract}




\section{Introduction}

Ischemic stroke is the second leading cause of death worldwide. One of the major risk factors is hypertension ${ }^{1}$, with diastolic blood pressure being a major determinant of outcome ${ }^{2}$. There is a gender specificity, with females having lower risk before menopause, but higher risk afterwards and in general a larger total burden in terms of mortality and morbidity due to stroke ${ }^{3,4}$.

Reactive oxygen species are believed to play a role in the pathogenesis of hypertension ${ }^{5-7}$. NADPH oxidases, with their sole function of ROS production and their abundant expression in the cardiovascular system have been implicated in hypertension ${ }^{8-11}$. Recently, a human study showed an upregulation of NOX5 in the kidney of hypertensive patients versus normotensives ${ }^{12}$. Also, NOX5 has been found to be expressed and active in endothelial ${ }^{13}$ and smooth muscle cells ${ }^{14}$ and it is upregulated by pro-hypertensive and pro-proliferative stimuli ${ }^{15,16}$. Rodents do not express NOX5, making it difficult to study the role of this isoform in in vivo animal models. We created a humanised NOX5 KI mouse, expressing the human NOX5 gene in vascular endothelial cells. We could therefore investigate the role of NOX5 in blood pressure regulation using in vivo telemetry and in ischemic stroke using the mouse tMCAO model.

\section{Results}

\section{Aged humanised eNOX5 KI mice show hypertensive phenotype}

Blood pressure was measured during 3 days in aged eNOX5 KI and matched WT mice (18-20 months) using a telemetry system. eNOX5 KI mice showed a higher systolic blood pressure (SBP) and mean arterial pressure (MAP) and a lower heart rate (Fig. 1A, B and D) without an accompanying change in diastolic blood pressure or activity (Fig. 1C and E). Average systolic pressure values over the 3 days were about $10 \mathrm{mmHg}$ higher in eNOX5 KI $(137 \pm 3 \mathrm{mmHg}, \mathrm{n}=33)$ versus WT $(127 \pm 2 \mathrm{mmHg}$, $n=31)(p<0.05)$. Gender specific analysis showed that SBP was increased in both males and females (data not shown), but MAP and diastolic pressure were only significantly elevated in females (Fig. 2). 
A
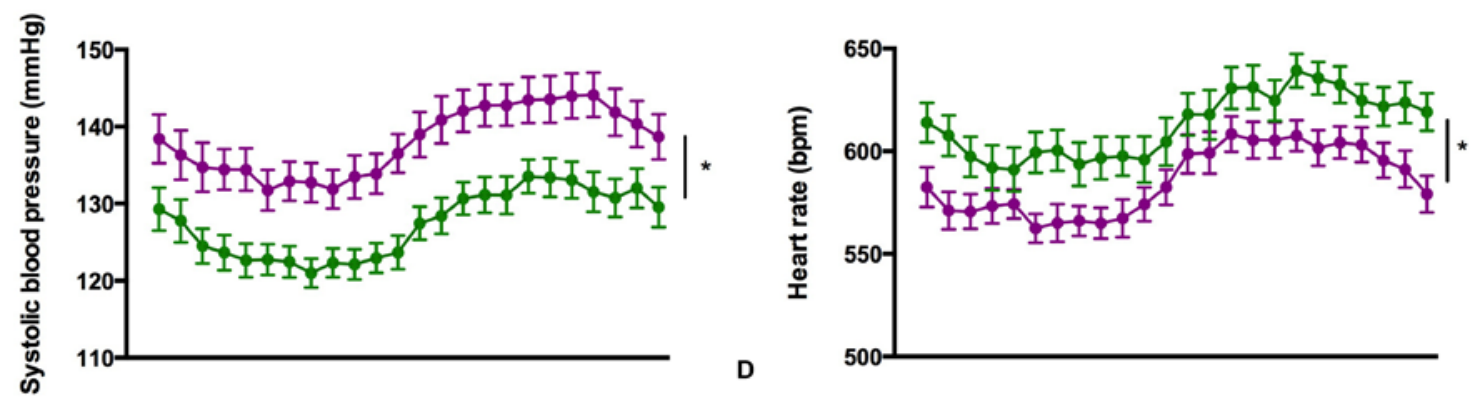

B
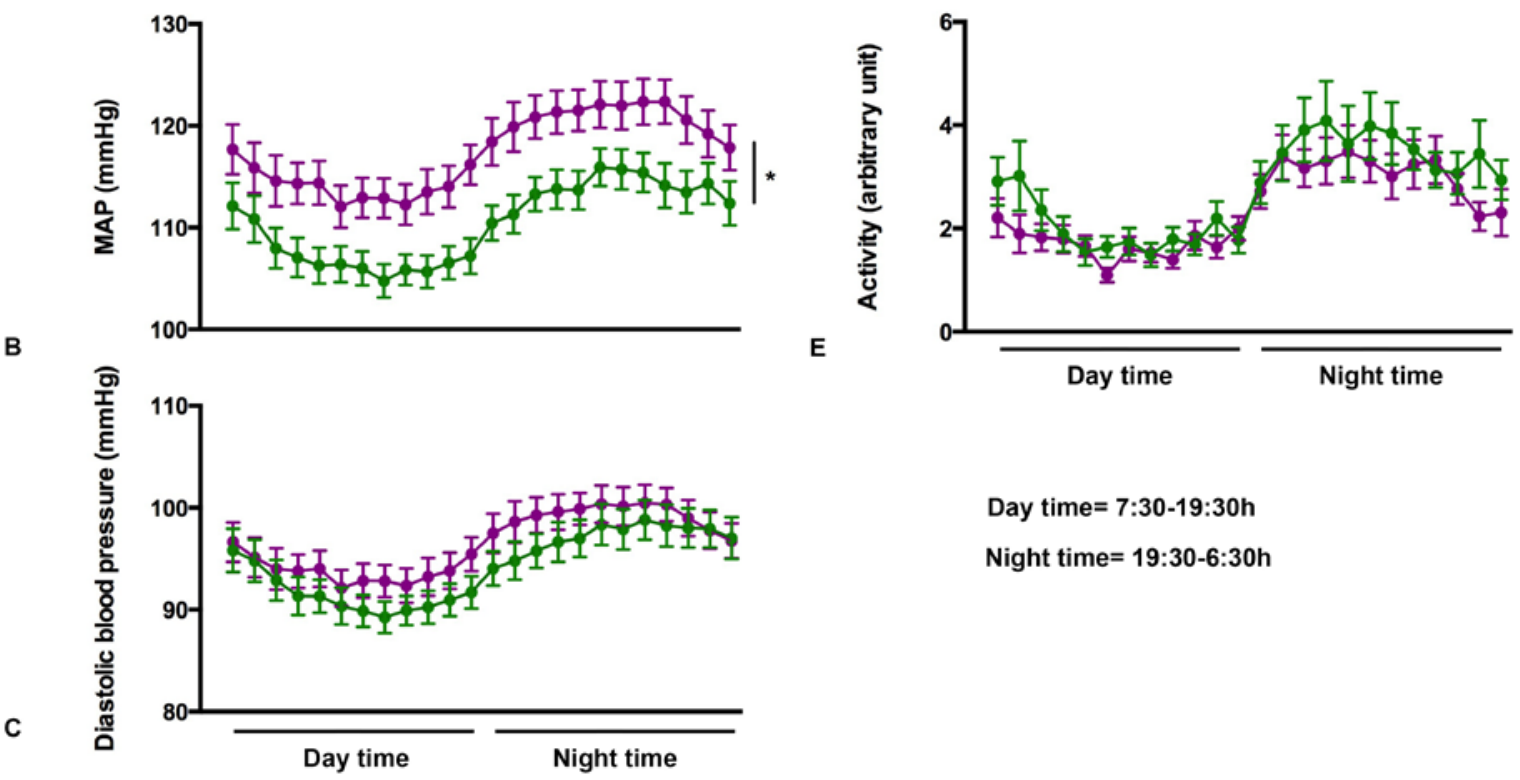

Day time $=7: 30-19: 30 \mathrm{~h}$

Night time $=19: 30-6: 30 \mathrm{~h}$

Figure 1. Aged NOX5 KI mice show a hypertensive phenotype. Blood pressure was measured for 3 consecutive days in aged eNOX5 KI ( $n=33$, purple lines) and WT ( $n=31$, green lines) mice. The values per hour were averaged over the three days. A) Systolic blood pressure was elevated in the eNOX5 KI mice compared to the WT. B) Mean arterial pressure was elevated in eNOX5 KI mice compared to the WT. C) Diastolic blood pressure was not changed in the eNOX5 KI mice. D) Heart rate was decreased in eNOX5 KI mice versus the WT mice. E) Activity levels were comparable between eNOX5 KI and WT mice. Data are expressed as mean $\pm S E M .{ }^{*} p<0.05 K I$ versus $W T$.

First results of blood pressure measurements in young eNOX5 KI and WT mice do not show a hypertensive phenotype (Fig. 3). 

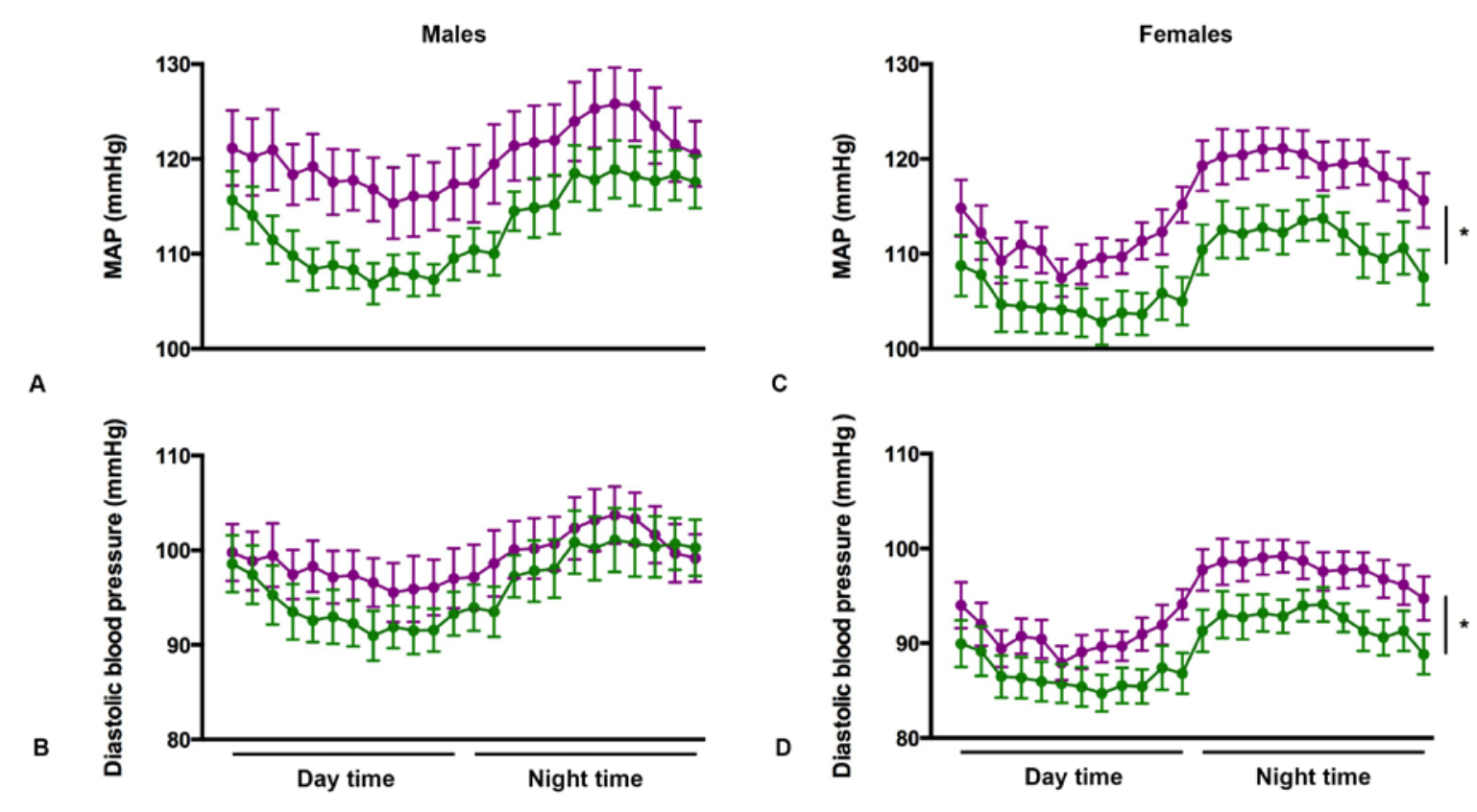

Figure 2. Gender specific increase in mean arterial pressure (MAP) and diastolic pressure in female eNOX5 KI (purple lines) versus female eNOX5 WT (green lines) mice. A and B) Mean arterial pressure $(A)$ and diastolic blood pressure $(B)$ were comparable in male eNOX5 KI $(n=15)$ and eNOX5 WT ( $n=15)$ mice. $C$ en D) Mean arterial pressure (C) and diastolic blood pressure $(D)$ were elevated in female eNOX5 KI $(n=18)$ compared to eNOX5 WT $(n=16)$ mice. Data are expressed as mean \pm SEM. ${ }^{*} p<0.05 \mathrm{KI}$ versus $W T$.

\section{Gender specific increased stroke size in young female NOX5 KI mice}

Eight to ten weeks old eNOX5 KI and WT mice were subjected to 30 minutes of brain ischemia using the tMCAO model. Infarct size as determined by TTC staining after 24 hours of reperfusion (Fig. 4A and B) did not show a difference between male eNOX5 KI $\left(52.5 \pm 7.5 \mathrm{~mm}^{3}, \mathrm{n}=18\right)$ and WT $\left(54.0 \pm 7.0 \mathrm{~mm}^{3}, \mathrm{n}=25\right)$ mice. In females however, infarct size was increased in eNOX5 KI mice $\left(\mathrm{KI} 97.7 \pm 10.9 \mathrm{~mm}^{3}, \mathrm{n}=12\right.$; WT $58.4 \pm 6.6, n=13, p<0.05)$. The increased infarct size was accompanied by a worse grip test score in the eNOX5 $\mathrm{KI}$ females (Fig. 4D). In males, the eNOX5 KI mice showed a worse (higher) Bederson score (Fig. 4C). 
A
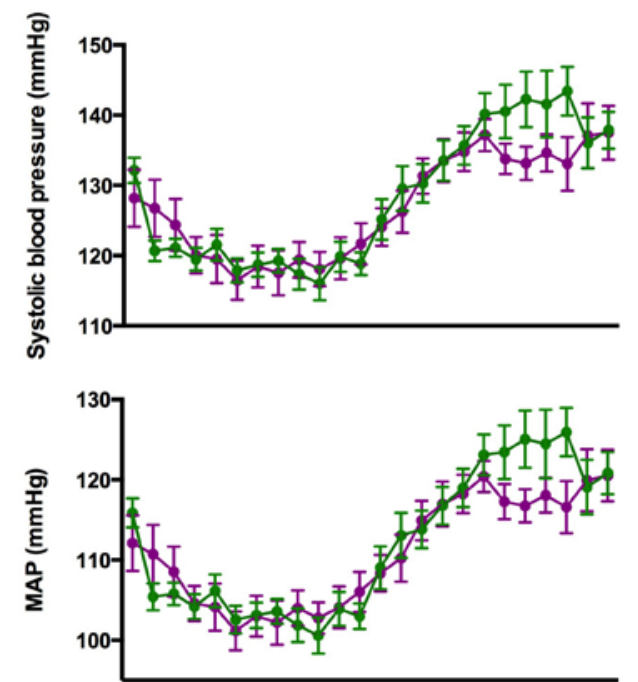

B

C
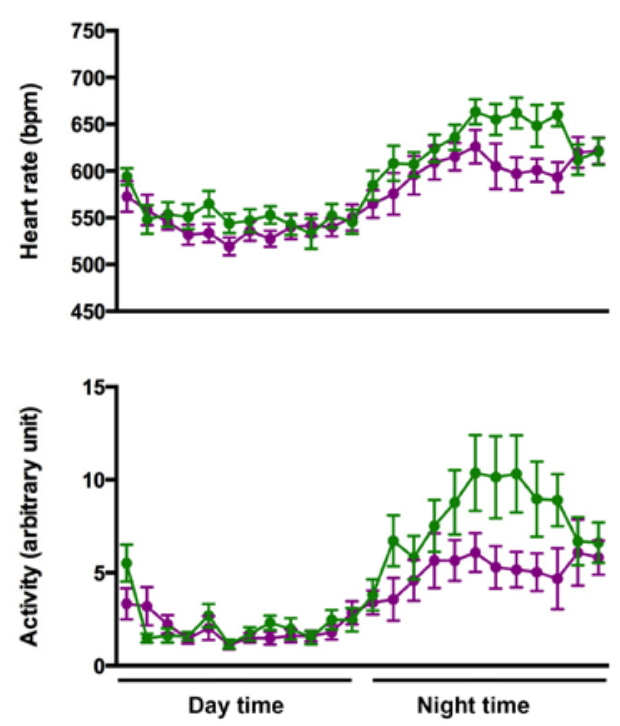

Day time $=7: 00-19: 00 \mathrm{~h}$

Night time=19:00-6:00h

Figure 3. No effect of NOX5 on blood pressure in young mice. Blood pressure was measured for 3 consecutive days in 8-12 weeks old eNOX5 KI ( $n=12$, purple lines) and WT $(n=12$, green lines) mice. The values per hour were averaged over the three days. A) Systolic blood pressure was comparable in the eNOX5 KI mice compared to the WT. B) Mean arterial pressure was comparable in eNOX5 KI mice compared to the WT. C) Diastolic blood pressure was not changed in the eNOX5 KI mice. D) Heart rate was comparable in eNOX5 $\mathrm{KI}$ mice versus the WT mice. E) Activity levels were comparable between eNOX5 KI and WT mice. Data are expressed as mean \pm SEM.

Blood brain barrier leakage was tested using Evans blue extravasation (Fig. 5). In the males, the eNOX5 KI mice $(5.4 \pm 0.8 \mathrm{ng} / \mathrm{mg}, \mathrm{n}=13)$ showed the same amount of Evans blue dye in the ipsilateral side of the brain as the WT $(5.4 \pm 0.6 \mathrm{ng} / \mathrm{mg})$. In the eNOX5 WT mice, more Evans blue extravasated in the ipsilateral (5.4 $\pm 0.6 \mathrm{ng} . \mathrm{mg})$ than in the contralateral side $(3.9 \pm 0.3 \mathrm{ng} / \mathrm{mg})$. In the male eNOX5 $\mathrm{KI}$ mice, this difference was not seen (ipsi 5.4 $\pm 0.8 \mathrm{ng} / \mathrm{mg}$; contra $4.3 \pm 0.4 \mathrm{ng} / \mathrm{mg}$ ). 
Female eNOX5 KI $(5.0 \pm 2.8 \mathrm{ng} / \mathrm{mg}, \mathrm{n}=9)$ mice showed an increased amount of Evans blue in the ipsilateral brain side compared to the WT $(3.4 \pm 0.3 \mathrm{ng} / \mathrm{mg})$ but this did not reach statistical significance $(p=0.057)$. Both the WT and the eNOX5 KI females showed more Evans blue in the ipsilateral versus the contralateral side, but this difference was more pronounced in the eNOX5 KI (WT ipsi 3.4 $\pm 0.3 \mathrm{ng} / \mathrm{mg}$; contra $2.5 \pm 0.1 \mathrm{ng} / \mathrm{mg} \mathrm{KI} \mathrm{ipsi} 5.1 \pm 0.8 \mathrm{ng} / \mathrm{mg}$; contra $2.8 \pm 0.2 \mathrm{ng} / \mathrm{mg}$ ).

A

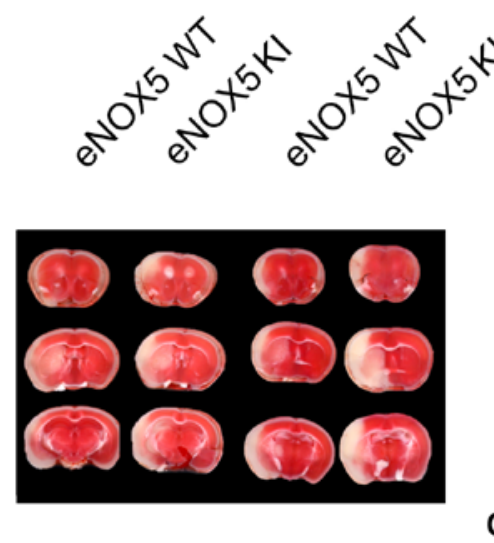

B

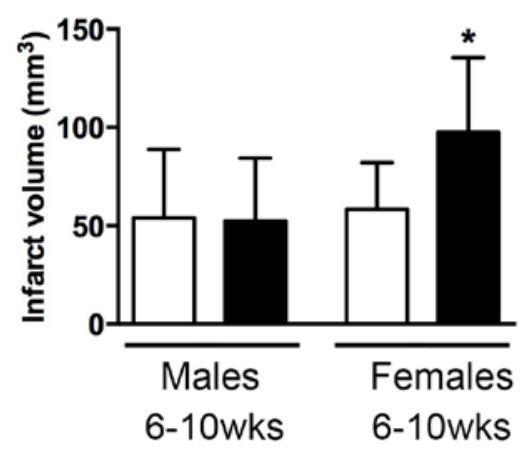

C
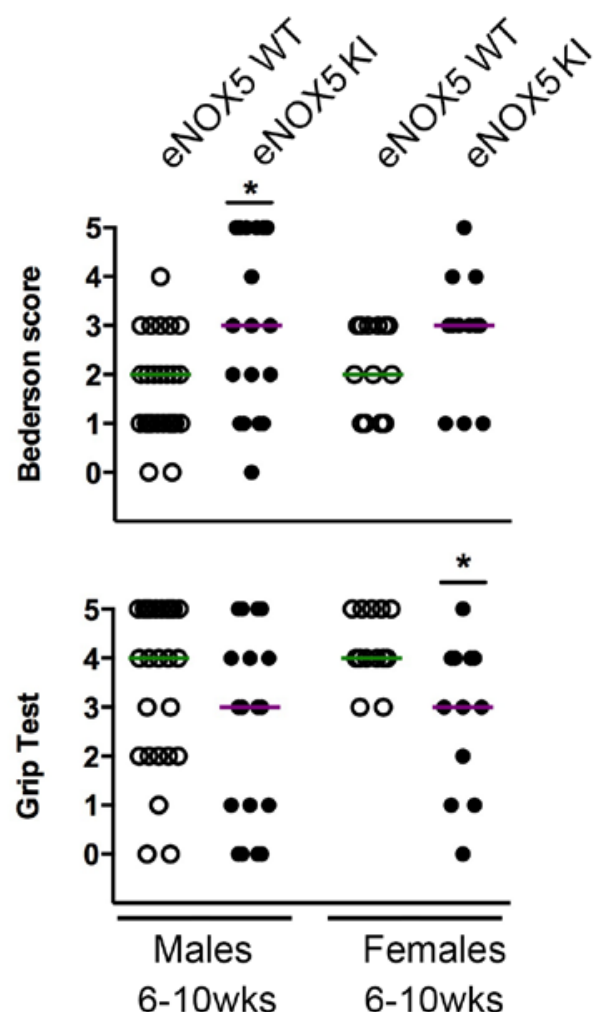

Figure 4. Gender specific effect of NOX5 on ischemic stroke. A) Representative TTC staining of three sequential coronal brain sections on day 1 after tMCAO The TTC staining colours viable tissue red, while infarcted tissue stays white. B) Infarct volumes in female eNOX5 KI $(n=12)$ were increased compared to eNOX5 WT $(n=13)$ females. Male eNOX5 KI $(n=18)$ infarct volumes were comparable to male WT $(n=25)$. Bars represent mean \pm SEM (white bars: eNOX5 WT mice, black bars: eNOX5 KI mice) C) Bederson scores ( $0=$ normal, $5=$ severe deficit) on day 1 after tMCAO were worse in male eNOX5 KI versus male WT mice. D) Grip test scores ( $0=$ severe deficit, $5=$ normal) were worse in eNOX5 KI females versus eNOX5 WT females. ( $C$ and D: open dots and green line: WT mice, closed dots and purple line: $K I$ mice). 
DHE staining showed an increased production of ROS in female eNOX5 KI (44.1 $\pm 0.6, n=4)$ mice when compared to eNOX5 WT females $(30.3 \pm 2.4, n=4)$ $(p<0.01)$ (Fig. 6), as expected when expressing the NOX5 gene that produces ROS.

A
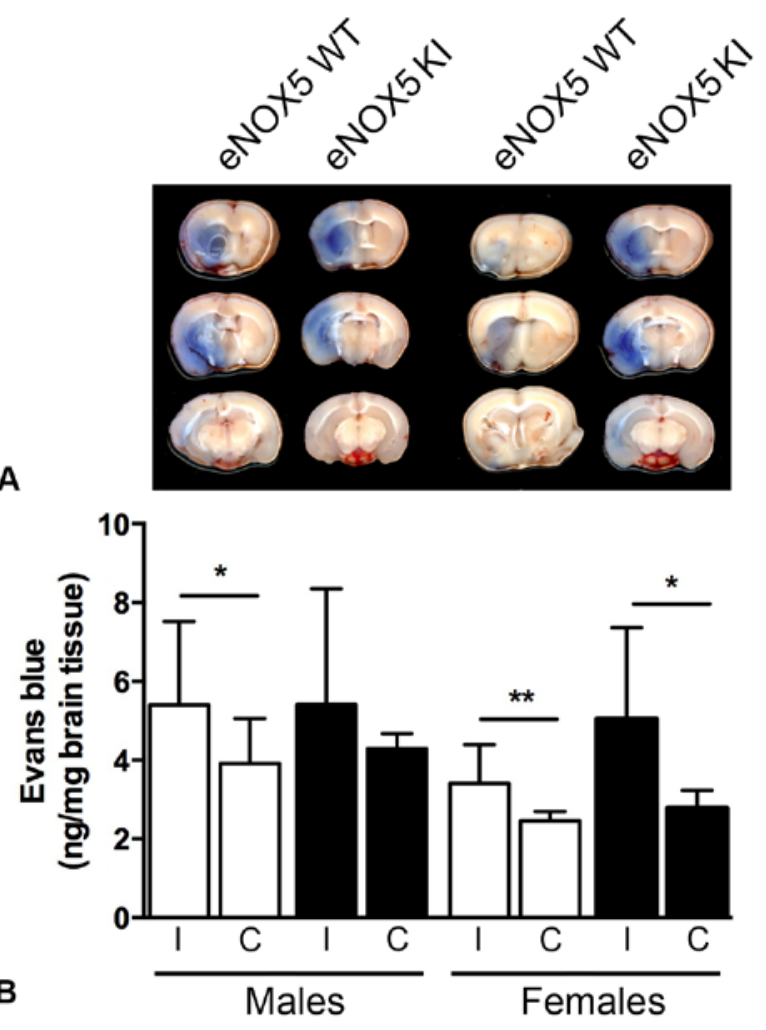

Figure 5. Blood brain barrier disruption in eNOX5 KI (black bars) and eNOX5 WT (white bars) mice. A) Representative pictures showing the extravasation of Evans blue dye into the ipsilateral and contralateral hemispheres of the brain 24h after IMCAO. B) Quantified amount of Evans blue dye extravasation (ng Evans blue/mg brain protein tissue) was increased in the ipsilateral versus contralateral brain side in eNOX5 WT females ( $n=10)$, eNOX5 KI females ( $n=9)$, eNOX5 WT males ( $n=13)$, but not in eNOX5 KI males $(n=14)$. Bars represent mean \pm SEM. $\left({ }^{*} p<0.05,{ }^{* *} p<0.01\right)$

\section{Discussion}

We show in a humanised endothelial cell specific NOX5 KI mouse model that NOX5 is responsible for systolic hypertension. Female eNOX5 $\mathrm{KI}$ mice additionally showed an increased diastolic blood pressure and an increased infarct size with enhanced blood brain barrier dysfunction when subjected to ischemic stroke. These data might point to NOX5 as a common denominator of both diseases. 


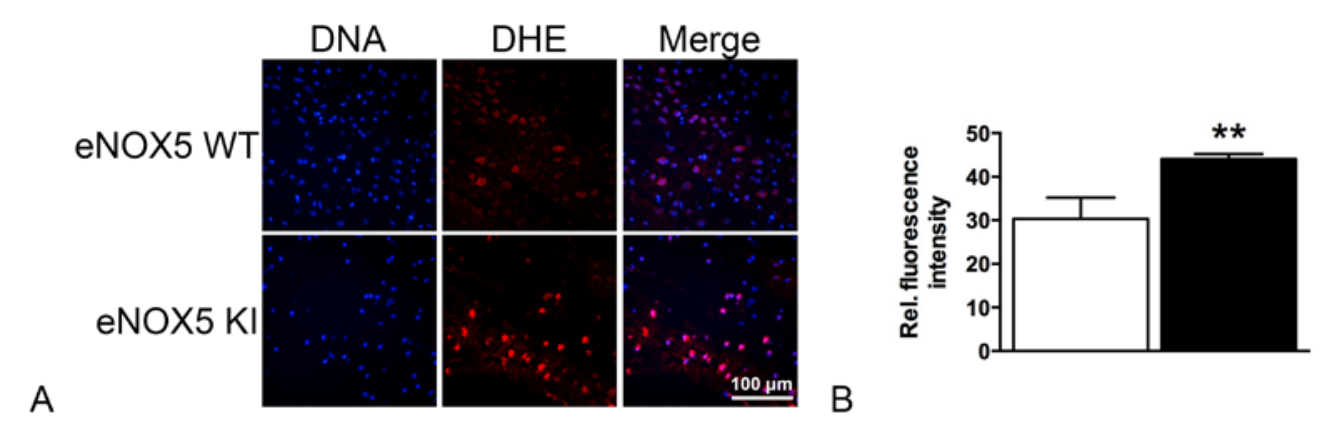

Figure 6. Increased oxidative stress in infarcted brains of the female eNOX5 KI mice.

A) Representative pictures of DHE and nuclear staining of the stroked brains. B) Relative DHE fluorescence intensity was increased in eNOX5 females (black bars, $n=4$ ) compared to eNOX5 WT females (white bars, $n=4$ ). Bars represent mean \pm SEM. ** $p<0.01$

Although females are somehow protected from stroke risk before menopause ${ }^{17}$, they catch up afterwards and the total burden of stroke is even higher in females than males ${ }^{4}$ with females having more severe stroke and higher case fatality rates ${ }^{18}$. Females who suffer from an ischemic stroke are more often diagnosed with having high blood pressure than males, making hypertension even a stronger risk factor in females. In our eNOX5 KI mice, diastolic blood pressure was only increased in the female mice. Diastolic hypertension is a main determinant of in-hospital mortality after stroke ${ }^{2}$. Our results suggest that if NOX5 is indeed a player in hypertension, and in females especially in diastolic hypertension, females would not only have a higher risk of developing stroke when hypertensive, they also would have increased mortality risk when diastolic hypertension occurs. NOX5 has been shown to be upregulated in renal cells of hypertensives ${ }^{12}$ and also in peripheral blood of hypertensive patients with micro-albuminuria (unpublished data). This makes our hypothesis of NOX5 being a key player in hypertension even stronger.

A possible mechanism to explain the hypertensive phenotype in our eNOX5 KI mice has not yet been elucidated. Stroke and hypertension share one common pathogenic mechanism, namely endothelial dysfunction. ROS have been implicated in endothelial dysfunction and thus it seems plausible that NOX5 could contribute to both stroke and hypertension via this mechanism. 
Another possible explanation for the hypertension would be renal dysfunction. NOX5 is expressed in the fetal kidney ${ }^{19}$ and in glomeruli of diabetic kidneys ${ }^{20}$. Podocyte specific NOX5 KI mice had diabetic and non-diabetic renal tubulo-interstitial fibrosis and damage and were hypertensive ${ }^{20}$. In addition, both ROS and NADPH oxidases have been described to be important in (renal) hypertension via various mechanisms (reviewed in ${ }^{5,21,22}$ ).

Thus, a role of NOX5 in the kidney seems very plausible. This possibility of renal involvement in the NOX5 hypertensive phenotype will be investigated in the near future.

\section{Methods}

\section{Animals}

All animal experiments were approved by the local animal experimental committee and performed according to the Dutch law on animal experiments. Animals were housed under controlled conditions $\left(22^{\circ} \mathrm{C}, 55-65 \%\right.$ humidity, $12 \mathrm{~h}$ light-dark cycle), and were allowed free access to water and standard laboratory chow. Male and female animals aged 6-10 weeks for the stroke and 18-22 months for the blood pressure were used. The NOX5 KI mice were compared to their matched WT's. For a description on the generation of the eNOX $5 \mathrm{KI}$ mice, please refer to Chapter 5 of this thesis.

\section{Blood pressure measurements}

Blood pressure was measured using telemetry devices (transmitters TA11PA-C10) of Data Sciences International and their Dataquest A.R.T software.

After administration of a painkiller (buprenorphine s.c. $0,05 \mathrm{mg} / \mathrm{kg}$, repeated every 12 hours), mice were anaesthetized with isoflurane (Abbott forene Isoflurane) $4-5 \%$ in air and then maintained with 2-3\% isoflurane in air via a vaporizor (Univentor, UNO Roestvaststaal BV).

The mouse was placed on a heating pad (UNO temperature control unit, UNO Roestvaststaal BV) and body temperature was monitored using a rectal probe and maintained at $37.0^{\circ} \mathrm{C}$ using a feedback-controlled infrared light. 
An incision in the skin overlying the carotid artery was made. Via this incision, in the subcutaneous space of the flank a pocket was created for inserting the telemetry transmitter (mice weighing $>25 \mathrm{gr}$ left side, $<25 \mathrm{gr}$ right side). The left carotid artery was dissected free and 3 ligatures (5-0, silk) were placed: at the bifurcation of the internal and external carotid to close the vessel, at the heart to temporarily close the vessel and one in between to fixate the catheter. Via a small hole cut in the artery, the catheter was introduced and advanced into the aortic arch. Then, the pocket in the flank was filled with $3 \mathrm{~mL}$ prewarmed saline and the transmitter was placed in the pocket. The wound was then closed using a polysorb 5-0 suture.

Mice were allowed to recover for 7-14 days before starting the measurement. Mice were housed individually in a quiet room. Blood pressure was measured over a $72 \mathrm{~h}$ period, with 10 cycles of $75 \mathrm{sec}$. per hour. The average per hour was calculated for the $72 \mathrm{~h}$ period.

\section{In vivo stroke model}

Please refer to Chapter 3 of this thesis for the description of the stroke model, infarct size and blood brain barrier integrity measurements, evaluation of neurological scoring and assessment of oxidative stress and apoptosis.

\section{References}

1. Lackland, D. T. et al. Factors influencing the decline in stroke mortality: a statement from the American Heart Association/American Stroke Association. Stroke 45, 315-353 (2014).

2. Caso, V. et al. High diastolic blood pressure is a risk factor for in-hospital mortality in complete MCA stroke patients. Neurol. Sci. 33, 545-549 (2012).

3. O'Donnell, M. J. et al. Risk factors for ischaemic and intracerebral haemorrhagic stroke in 22 countries (the INTERSTROKE study): a casecontrol study. Lancet 376, 112-123 (2010).

4. Bushnell, C. et al. Guidelines for the prevention of stroke in women: a statement for healthcare professionals from the American Heart Association/American Stroke Association. Stroke 45, 1545-1588 (2014). 
5. Harrison, D. G., Gongora, M. C., Guzik, T. J. \& Widder, J. Oxidative stress and hypertension. J Am Soc Hypertens 1, 30-44 (2007).

6. Griendling, K. K. \& FitzGerald, G. A. Oxidative stress and cardiovascular injury: Part II: animal and human studies. Circulation 108, 2034-2040 (2003).

7. Elahi, M. M., Kong, Y. X. \& Matata, B. M. Oxidative stress as a mediator of cardiovascular disease. Oxid Med Cell Longev 2, 259-269 (2009).

8. Wind, S. et al. Oxidative stress and endothelial dysfunction in aortas of aged spontaneously hypertensive rats by NOX1/2 is reversed by NADPH oxidase inhibition. Hypertension 56, 490-497 (2010).

9. Ray, R. et al. Endothelial nox4 NADPH oxidase enhances vasodilatation and reduces blood pressure in vivo. Arterioscler Thromb Vasc Biol 31, 1368-1376 (2011).

10. Lassegue, B. \& Griendling, K. K. NADPH oxidases: functions and pathologies in the vasculature. Arterioscler Thromb Vasc Biol 30, 653-661 (2010).

11. Cave, A. C. et al. NADPH oxidases in cardiovascular health and disease. Antioxid Redox Signal 8, 691-728 (2006).

12. Yu, P. et al. Unique role of NADPH oxidase 5 in oxidative stress in human renal proximal tubule cells. Redox Biol 2, 570-579 (2014).

13. BelAiba, R. S. et al. NOX5 variants are functionally active in endothelial cells. Free Radic Biol Med 42, 446-459 (2007).

14. Pandey, D. et al. Expression and functional significance of NADPH oxidase 5 (Nox5) and its splice variants in human blood vessels. Am. J. Physiol. Heart Circ. Physiol. 302, H1919-28 (2012).

15. Jay, D. B. et al. Nox5 mediates PDGF-induced proliferation in human aortic smooth muscle cells. Free Radic Biol Med 45, 329-335 (2008).

16. Montezano, A. C. et al. Nicotinamide adenine dinucleotide phosphate reduced oxidase 5 (Nox5) regulation by angiotensin II and endothelin-1 is mediated via calcium/calmodulin-dependent, rac-1-independent pathways in human endothelial cells. Circ Res 106, 1363-1373 (2010).

17. Murphy, S. J., McCullough, L. D. \& Smith, J. M. Stroke in the female: role of biological sex and estrogen. ILAR J 45, 147-159 (2004).

18. Appelros, P., Stegmayr, B. \& Terént, A. Sex differences in stroke epidemiology: a systematic review. Stroke 40, 1082-1090 (2009). 
19. Cheng, G., Cao, Z., Xu, X., van Meir, E. G. \& Lambeth, J. D. Homologs of gp91phox: cloning and tissue expression of Nox3, Nox4, and Nox5. Gene 269, 131-140 (2001).

20. Holterman, C. E. et al. Nephropathy and Elevated BP in Mice with PodocyteSpecific NADPH Oxidase 5 Expression. J. Am. Soc. Nephrol. (2013). doi:10.1681/ASN.2013040371

21. Montezano, A. C. \& Touyz, R. M. Oxidative stress, Noxs, and hypertension: experimental evidence and clinical controversies. Ann. Med. 44 Suppl 1, S216 (2012).

22. Araujo, M. \& Wilcox, C. S. Oxidative stress in hypertension: role of the kidney. Antioxid Redox Signal 20, 74-101 (2014). 
Chapter 7

General discussion and conclusion 
Reactive oxygen species play a major role in ischemia-reperfusion injury. With respect to a therapeutic exploitation of this, anti-oxidant approaches have been proven to be ineffective for different disease states. In this thesis, we explored a different approach by identifying and targeting disease relevant enzymatic sources of ROS. With respect to such a drugable source, the focus was on NADPH oxidases, the only known dedicated ROS source in the body. Using different models of ischemia-reperfusion injury in several genetically modified mice, we successfully answered all four scientific questions phrased in the introduction:

\section{Regarding the detrimental role of NOX4 in stroke, which vascular cell type that expresses NOX4 is responsible?}

We identified endothelial cell NOX4 as the major vascular cell type responsible for the detrimental ROS production in stroke. eNOX4 KO mice show a decreased infarct size, better neurological scoring and better preserved blood brain barrier integrity as their matched WT's. Smooth muscle cell specific NOX4 KO mice did not show any protection of ischemic stroke, excluding VSMC NOX4 as key player. The effect seen in the mice lacking endothelial NOX4 was smaller than the effect seen in the global NOX4 KO, pointing towards a role of an additional cell type expressing NOX4. In vitro, we could show that neurons treated with the NOX inhibitor GKT or VAS showed reduced apoptosis after oxygen glucose deprivation. These data indicate that neuronal NOX4 could be the additional player in ischemia of the brain. Currently, in vivo experiments using neuronal NOX4 $\mathrm{KO}$ mice are being performed to further elucidate this possibility.

\section{Can quality or power issues explain the disagreement on the role of NOX2 in preclinical stroke research?}

With respect to NADPH oxidases, several papers showed a detrimental role of NOX2 in ischemic stroke, while our own group could not reproduce these findings. A systematic review and meta-analysis now show insufficient reporting, risk of bias and a publication bias in the available literature on NOX2 in ischemic stroke. Moreover, most of the studies were underpowered to detect a (according to us) clinically relevant infarct size reduction of $40 \%$. 
Subsequently, in a randomised, blinded, sufficiently powered, preclinical trial we could show that NOX2 has only a very minor effect on infarct size, for which $>200$ animals per group would have to be included to power this as a significant finding. In addition, no effect on the clinically more relevant neurological scoring was found. Thus, these findings exclude NOX2 as a major target for pharmacological treatment in ischemic stroke.

\section{Does NOX4 play a role in ischemia-reperfusion injury in other organs than the brain?}

Since NOX4 is induced by hypoxia (via HIF-1 alpha) in other organs we were interested whether its role in IRI could be extended to heart and periphery. Using permanent and transient cardiac ischemia, however, no role of NOX4 on the chronic response in the ischemic heart was found, excluding this isoform as possible therapeutic target. Also, NOX1, 2 and 5 were investigated with only minor findings leading to the conclusion that also these isoforms are not relevant in ischemic heart disease. As a second peripheral organ, the hindlimb was subjected to ischemia as a model of peripheral artery disease. Again, no role for any of the NADPH oxidase isoforms could be shown. For NOX4 thus this means that it has a brain specific effect in ischemia-reperfusion injury. We can also conclude that there are time-, cell- and isoform specific effects of the other NADPH oxidases.

\section{Can the role of NOX in stroke be translated into the clinic?}

With respect to IRI and NOX, we therefore returned our focus to the brain. One isoform had been overlooked or under-investigated so far, NOX5. This gene is not expressed in mice and rats and was therefore hardly studied up to now. In human hypertension, NOX5 is upregulated in renal tubular cells ${ }^{1}$. Hypertension is also the major risk factor for stroke. We showed that NOX5 KI mice are hypertensive when aged, with only females also having diastolic hypertension. Additionally, the NOX5 KI females showed larger infarct sizes and blood brain barrier disruption after ischemic stroke when compared to NOX5 WT females. This was not seen in male NOX5 KI mice. 
These results suggest that NOX5 might be a common player in both hypertension and stroke, with endothelial dysfunction as the underlying pathomechanism. The overlooked NOX5 thus seems to be a second NOX-target, besides NOX4, for neuroprotective therapy in stroke. Clearly, the next steps in late-stage pre-clinical development will be to validate NOX4/5 in a second small animal (e.g. rat) and in a large animal model before considering clinical trials with phase I tested NOX inhibitor compounds.

During the course of this thesis, other important issues became apparent, which are very relevant to the problem of translational failures of biomedical research in general.

Many preclinical promising treatments eventually fail in the clinic. Animal studies show major benefit, but in patients eventually, no effect is found. Stroke is a major example of this with more than thousand drug candidates failing in the clinic ${ }^{2}$. Does this make preclinical research unnecessary? No, but several quality points need attention to enhance clinical translational success:

\section{Translational failure: mice are not humans}

External validity, the generalizability of a study, should be considered when doing animal experiments ${ }^{3}$. Straightforward clinical translation of animal experiments is not possible ${ }^{4}$. It is obvious that mice are not humans thus there is already a possible intrinsic error by using animals as models for humans. Also, the animal models used do not always exactly mimic the human clinical situation ${ }^{5-8}$. When designing animal experiments, one should consider carefully the characteristics of the animals/patients. Often, young and healthy male mice are used, while in the clinics, patients are of both genders, are old(er) and have several comorbidities that might influence outcome ${ }^{9}$. Dosing and timing of the treatment is also important. With stroke, for example, there is a huge delay in the clinic between onset of symptoms and treatment, while animal studies often immediately start the treatment ${ }^{10}$. Drug metabolism differs between humans and mice, making it difficult to use a comparable dosage in mice as in humans ${ }^{11}$. Over -or underdosing could lead to better responses or fewer side effects in mice. 
Choosing the right outcome measures is a last point in external validity ${ }^{3,12}$. Measurements in animals should represent the major clinical end points and also the timing of these end-points. With stroke for example, measuring infarct size is maybe the most obvious possibility, but in clinics, neurological outcome and functioning are much more important, independent of the infarct size.

\section{Translational failure: pitfalls in internal validity}

In addition to low external validity, also the internal validity of preclinical studies should be considered to enhance translational success ${ }^{13-15}$ Just as in randomised controlled clinical trials, biases should be prevented. Blinding is a first important step. Of course, blinding of the 'patients', in this case the animals to prevent performance bias is not possible. However, random housing and blinding of the caretakers can help in this. Blinding of all persons involved in the treatment of animals, data gathering and data analysis should be implemented to prevent detection bias ${ }^{16}$. Randomisation of the included animals will create groups with comparable baseline characteristics, thus preventing selection bias. As last, attrition bias can be prevented by reporting the number and reasons of dropouts. Reporting of all the bias-prevention points mentioned above is also one of the quality measures that need to be taken to make animal research more like RCT's ${ }^{17}$. Several initiatives have already been started to increase the quality of animals studies in general ${ }^{18,19}$ and stroke specifically ${ }^{20-22}$. In conclusion, to enhance clinical translatability, RCT's should be taken as an example for preclinical research.

\section{Take home messages}

This thesis firstly identifies the ROS producing enzymes NOX4 and NOX5 as major players in stroke and hypertension. Future research might lead to the first neuroprotective treatment for this devastating disease. In addition, the thesis shows that preclinical research needs better guidelines and higher quality to enhance the translatability of research from bench to bedside. 


\section{References}

1. $\mathrm{Yu}, \mathrm{P}$. et al. Unique role of NADPH oxidase 5 in oxidative stress in human renal proximal tubule cells. Redox Biol 2, 570-579 (2014).

2. O'Collins, V. E. et al. 1,026 experimental treatments in acute stroke. Ann. Neurol. 59, 467-477 (2006).

3. van der Worp, H. B. et al. Can animal models of disease reliably inform human studies? PLoS Med. 7, e1000245 (2010).

4. McGonigle, P. \& Ruggeri, B. Animal models of human disease: challenges in enabling translation. Biochem. Pharmacol. 87, 162-171 (2014).

5. Ruggeri, B. A., Camp, F. \& Miknyoczki, S. Animal models of disease: preclinical animal models of cancer and their applications and utility in drug discovery. Biochem. Pharmacol. 87, 150-161 (2014).

6. McGonigle, P. Animal models of CNS disorders. Biochem. Pharmacol. 87, 140-149 (2014).

7. Mullane, K. \& Williams, M. Animal models of asthma: reprise or reboot? Biochem. Pharmacol. 87, 131-139 (2014).

8. Webb, D. R. Animal models of human disease: inflammation. Biochem. Pharmacol. 87, 121-130 (2014).

9. Sena, E., van der Worp, H. B., Howells, D. \& Macleod, M. How can we improve the pre-clinical development of drugs for stroke? Trends Neurosci. 30, 433-439 (2007).

10. van der Worp, H. B., de Haan, P., Morrema, E. \& Kalkman, C. J. Methodological quality of animal studies on neuroprotection in focal cerebral ischaemia. J. Neurol. 252, 1108-1114 (2005).

11. Wagner, D.-C. et al. Allometric dose retranslation unveiled substantial immunological side effects of granulocyte colony-stimulating factor after stroke. Stroke 45, 623-626 (2014).

12. Gladstone, D. J., Black, S. E., Hakim, A. M.Heart and Stroke Foundation of Ontario Centre of Excellence in Stroke Recovery. Toward wisdom from failure: lessons from neuroprotective stroke trials and new therapeutic directions. Stroke 33, 2123-2136 (2002). 
13. Kilkenny, C. et al. Survey of the quality of experimental design, statistical analysis and reporting of research using animals. PLOS ONE 4, e7824 (2009).

14. Dirnagl, U. Bench to bedside: the quest for quality in experimental stroke research. J Cereb Blood Flow Metab 26, 1465-1478 (2006).

15. Braeuninger, S. \& Kleinschnitz, C. Rodent models of focal cerebral ischemia: procedural pitfalls and translational problems. Exp Transl Stroke Med 1, 8 (2009).

16. Crossley, N. A. et al. Empirical evidence of bias in the design of experimental stroke studies: a metaepidemiologic approach. Stroke 39, 929-934 (2008).

17. Landis, S. C. et al. A call for transparent reporting to optimize the predictive value of preclinical research. Nature 490, 187-191 (2012).

18. Hooijmans, C. R., Leenaars, M. \& Ritskes-Hoitinga, M. A gold standard publication checklist to improve the quality of animal studies, to fully integrate the Three Rs, and to make systematic reviews more feasible. Altern Lab Anim 38, 167-182 (2010).

19. Kilkenny, C., Browne, W. J., Cuthill, I. C., Emerson, M. \& Altman, D. G. Improving bioscience research reporting: the ARRIVE guidelines for reporting animal research. PLoS Biol. 8, e1000412 (2010).

20. Boltze, J., Ayata, C., Wagner, D.-C. \& Plesnila, N. Preclinical phase III trials in translational stroke research: call for collective design of framework and guidelines. Stroke 45, 357-357 (2014).

21. Dirnagl, U. \& Fisher, M. International, multicenter randomized preclinical trials in translational stroke research: it's time to act. J Cereb Blood Flow Metab 32, 933-935 (2012).

22. Stroke Therapy Academic Industry Roundtable (STAIR). Recommendations for standards regarding preclinical neuroprotective and restorative drug development. Stroke 30, 2752-2758 (1999). 
Chapter 8

Summary 
Myocardial infarction, stroke and peripheral artery disease present a high mortality, morbidity and disability burden for society. They appear to share a common underlying process in which reactive oxygen species (ROS) are key players.

With respect to utilising this pathomechanistic insight in a therapeutic manner, scavenging ROS with antioxidants did not result in clinical benefit. Most likely this was due to the fact that ROS fulfil also essential signalling roles.

Here a different, more promising approach is pursued: preventing already the formation of damaging ROS by inhibiting the disease-relevant enzyme, NADPH oxidase (NOX), and at the same time leaving physiological ROS formation from other sources untouched.

Several vascular forms of NOX exist $(1,2,4$ and 5). In this thesis NOX4 and 5 are identified and validated as new targets for stroke therapy. Surprisingly, this approach is absolutely specific for the brain. Despite the common underlying oxidative stress, in myocardial infarction and peripheral artery disease, no role of any NOX was found in these conditions. For NOX4, even cell-specificity could be established, suggesting a predominant role in endothelial apoptosis and blood-brain-barrier breakdown.

In the course of these studies we found discrepancies between our own findings and the literature. We discovered a publication bias leading to an over-representation of positive, often statistically underpowered studies. Thus research quality became another aspect of this thesis and concludes in a call for randomised controlled trials also at the pre-clinical level (pRCT). Such an approach will enhance the translatability of preclinical research in general.

Collectively, NADPH oxidases have cell- and disease-specific effects. NOX4 and 5 were identified as novel targets in stroke. Further development may now lead to the first ever neuroprotective therapy for this devastating condition. 
Chapter 9

Samenvatting 
Hartinfarct, beroerte en perifeer vaatlijden leggen een hoge last op de maatschappij in termen van mortaliteit, morbiditeit en invaliditeitslast. Ze lijken eenzelfde onderliggend proces te delen, waarin reactieve zuurstof deeltjes (ROS) een sleutelrol spelen.

Met betrekking tot het gebruik van dit pathomechanistische inzicht leidde het wegvangen van ROS door antioxidanten, niet tot klinische verbetering. Dit is waarschijnlijk te wijten aan het feit dat ROS ook essentiële signaleringsrollen vervullen.

Hier wordt een andere veelbelovende benadering nagestreefd: de vorming van schadelijke ROS tegengaan door het remmen van het ziekte-relevante enzym NADPH oxidase (NOX), waarbij tegelijkertijd de fysiologische ROS vorming uit andere bronnen onaangeroerd blijft.

Er bestaan verschillende vasculaire vormen van $\operatorname{NOX}(1,2,4$ en 5). In dit proefschrift worden NOX4 en 5 geïdentificeerd en gevalideerd als nieuwe aangrijpingspunten voor de behandeling van een beroerte. Verrassenderwijs blijkt deze benadering specifiek voor de hersenen te zijn. Ondanks de overeenkomstige onderliggende oxidatieve stress bij een myocardinfarct en perifeer vaatlijden, werd onder deze omstandigheden géén rol van NOX aangetoond. Voor NOX4 kon zelfs celspecificiteit worden vastgesteld, hetgeen duidt op een overheersende rol in endotheliale apoptose en beschadiging van de bloed-hersen-barrière.

Tijdens deze studies kwamen verschillen tussen onze bevindingen en de literatuur aan het licht. We ontdekten een publicatiebias die leidt tot een oververtegenwoordiging van positieve studies met vaak een te lage statistische power. Kwaliteit van onderzoek werd zo een ander aspect van dit proefschrift en eindigt in een oproep voor gerandomiseerde gecontroleerde studies ook op preklinisch niveau (pRCT). Een dergelijke aanpak zal de vertaalbaarheid van preklinisch onderzoek in het algemeen verbeteren. 
In conclusie worden cel- en ziekte-specifieke effecten van NADPH oxidasen beschreven. NOX4 en 5 worden geïdentificeerd als nieuwe aangrijpingspunten bij een beroerte. Verdere ontwikkeling zou vervolgens kunnen leiden tot de eerste neuroprotectieve behandeling ooit voor deze verwoestende aandoening. 
Chapter 10

Valorisation 
Cardiovascular diseases (CVD) are the leading cause of mortality worldwide (17 million deaths per year) with an increasing incidence during the last decades. In addition, many patients suffer daily from the consequences of cardiovascular diseases.

Stroke and myocardial infarction are two major entities within the total group of CVD, with many patients being disabled after such an event. Treatment of these events is suboptimal, only symptomatic treatment is possible, trying to reduce the damage that is already there. For stroke, there is even only 1 drug approved, with so many contraindications that $>85 \%$ of patients is left untreated. New treatments are thus necessary to reduce the total burden of CVD on individual patients and society.

In recent years, antioxidants have been described as being good for your health and antioxidants are being sold as preventative food supplements. In cardiovascular research, antioxidants have been given a large amount of attention also as possible drug treatments for stroke, myocardial infarction and other cardiovascular diseases. The formation of damaging oxygen radicals (reactive oxygen species (ROS)) is the basis of almost all cardiovascular disease and antioxidants have been used as an approach to scavenge these ROS and thus reduce damage. However, in large clinical trials, the effects of antioxidants could not be proven; on the contrary, they even can be harmful! Thus, new approaches are needed to find a breakthrough in the treatment of these devastating diseases.

In this thesis, one of these new approaches is addressed. Instead of scavenging the ROS after they have been formed, we try to identify the sources that produce the ROS and try to inhibit them. NADPH oxidases are proteins that are the major source of ROS production within the cardiovascular system and these proteins are thus the focus of our current research. Several different forms of these proteins exist (NOX1-5) which differ in the location in the body where they are expressed, their activation and the sort of ROS they produce. Using animal models, where we specifically delete one of these isoforms in the genome, we could show that NOX4, but not NOX1 and 2 are important for the damage to the brain during a stroke. We could even identify one specific cell type expressing this NOX4 that is responsible for the damaging ROS. 
In a model of myocardial infarction and also a model of peripheral artery disease, no role of these NADPH oxidases was found. So, apparently there is a brain specific effect. Also, NOX5 was found to be related to high blood pressure in general and stroke in females, making this form of NOX also a possible therapeutic target.

These findings open up new doors for treatment of stroke and possibly other cardiovascular disease. Unravelling underlying pathways and problems is the first step in the process of new drug development. However, before the step to clinics can be made, far more preclinical research is needed. Extending our findings to a second small animal species (rats) and later larger animals is a first step. In addition we need to keep searching for a compound that has a good tolerability and safety profile to be used in clinical phase II trials.

Using the new approach of tackling the root of a pathophysiological process can also be generalised to other fields of research. A lot of diseases seem to be interconnected. The term 'diseasome' (see Fig. 1, from ${ }^{1}$ ) has been introduced recently, showing the possible mechanistic links between different disease entities. Some drugs used already in clinic for one condition, might thus also be useful in treating other, connected diseases. Finding out more about the specific underlying disease mechanisms can ultimately lead to personalised medicine, treating a patient, not a disease. Preventing diseases is another possibility with tackling the root of the problem. NADPH oxidases, with their specific function of ROS production that lead to several diseases, seem to be an ideal target for this, at least in stroke. In addition, the specific findings on the NADPH oxidases can also be extended to other diseases. Perinatal hypoxia, for example, is an ischemic condition of the newborn brain which has a lot of resemblance with adult stroke. Investigating the role of NADPH oxidases in this condition might also there lead to new therapeutic targets. Extending the knowledge to other neurodegenerative disease, such as neurotrauma may even make more patients benefit from this approach. For this, collaboration between researchers from different fields is stimulated, leading to more knowledge and technique transfer, also with respect to this growing diseasome. Every research field can contribute then its own small part of the very large puzzle. 


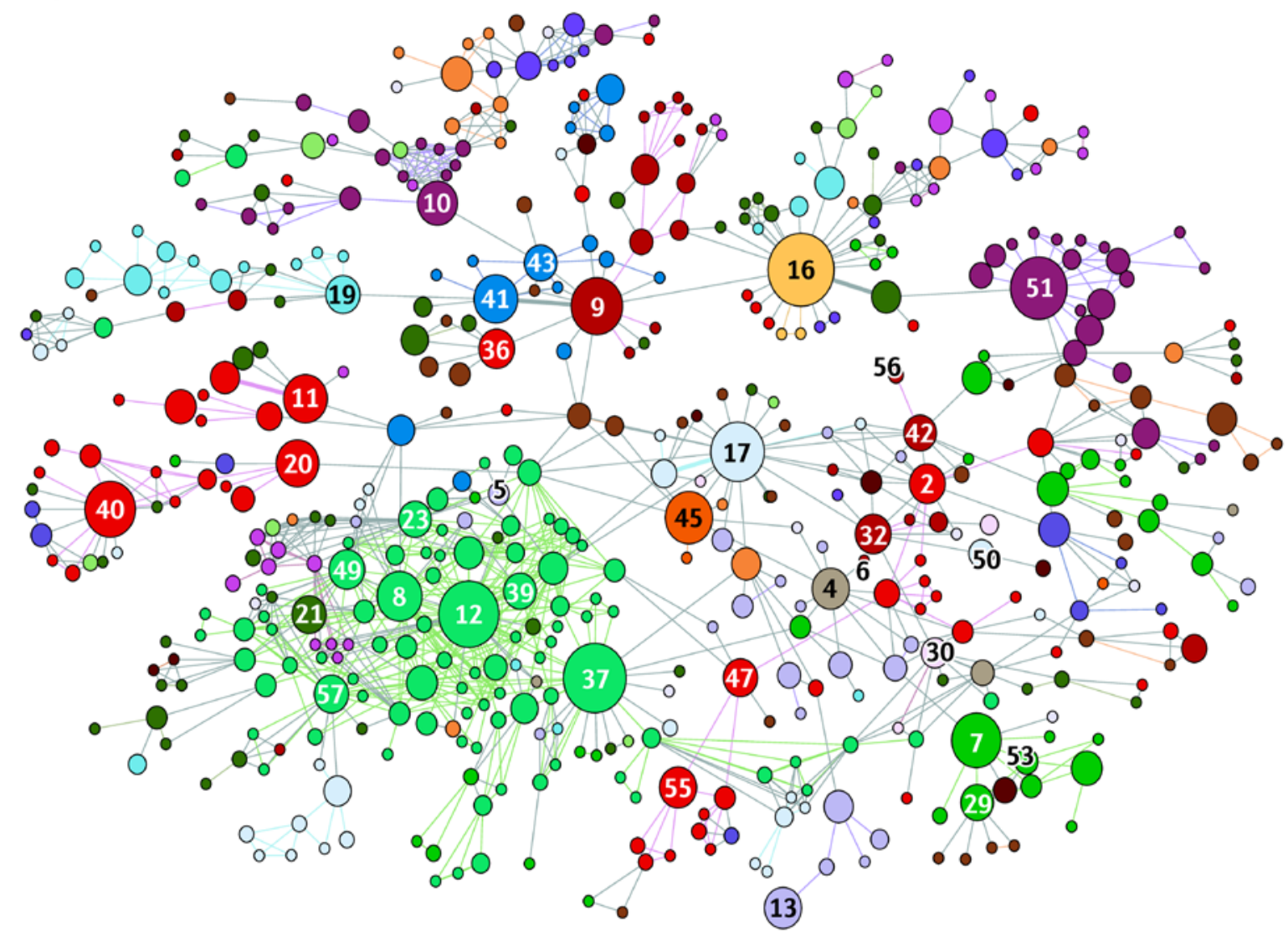

\begin{tabular}{|c|c|c|}
\hline (1) Aldosteronism & (20) Epilepsy & (42) Myocardial infarction \\
\hline (2) Alzheimer's disease & (21) Fanconi's anaemia & (43) Myopathy \\
\hline (3) Anaemia, congenital & $\begin{array}{l}\text { (22) Fatty liver } \\
\text { (23) Gastric cancer }\end{array}$ & (44) Nucleoside phosphorylase \\
\hline (4) Asthma & (24) Gilbert's syndrome & (45) Obesity \\
\hline (5) Ataxia-telangiectasia & (25) Glaucoma 1A & (46) Paraganglioma \\
\hline (6) Atherosclerosis & (26) Goitre congenital & (47) Parkinson's disease \\
\hline (7) Blood group & (27) HARP syndrome & (48) Pheochromocytoma \\
\hline (8) Breast cancer & (28) HELLP syndrome & (49) Prostate cancer \\
\hline (9) Cardiomyopathy & (29) Haemolytic anaemia & (50) Pseudohypoaldosteronism \\
\hline (10) Cataract & (30) Hirschprung disease & (51) Retinitis pigmentosa \\
\hline (11) Charcot-Marie-Tooth & (31) Hyperbilirubinaemia & (52) Schizoaffective disorder \\
\hline disease & (32) Hypertension & (53) Spherocytosis \\
\hline (12) Colon cancer & (33) Hypertension diastolic & (54) Spina bifida \\
\hline (13) Complement component & (34) Hyperthyroidism & (55) Spinocerebellar ataxia \\
\hline deficiency & (35) Hypoaldosteronism & (56) Stroke \\
\hline (14) Coronary artery disease & (36) Leigh syndrome & (57) Thyroid carcinoma \\
\hline (15) Coronary spasm & (37) Leukaemia & (58) Total iodide organification \\
\hline (16) Deafness & (38) Low renin hypertension & defect \\
\hline (17) Diabetes mellitus & (39) Lymphoma & (59) Trifunctional protein \\
\hline (18) Enolase- $\beta$ deficiency & (40) Mental retardation & deficiency \\
\hline (19) Epidermolysis bullosa & (41) Muscular dystrophy & (60) Unipolar depression \\
\hline
\end{tabular}

Figure 1. The diseasome (from Barabasi et al ${ }^{1}$ ) 
One pitfall of the approach of targeting NADPH oxidases, is that they also produce ROS that are needed by the body for its normal functioning. ROS are for example needed for the immune response and for the formation of blood vessels. This is also one of the reasons why unspecific scavenging of ROS by anti-oxidants might have failed. Moreover, as we have shown, not every form of the NADPH oxidases plays the same role in the same disease. The need for specific NADPH inhibitors provides possibilities for industry. Small spin-off companies and larger industry partners can use the new mechanistic target(s) for developing specific drugs. If the underlying mechanisms are really known, this will automatically also lead to less side effects which is again beneficial for the patient. The inhibitors that are produced in industry then, and also the knowledge that is gained can be shared with researchers.

In this way, also cooperations between academia and industry can be initiated and enhanced, working together towards a personalised medicine approach.

Another major pitfall of the approach that we adhere to, is the poor translatability from preclinical animal models to the human clinical situation. These issues are also addressed in this thesis, since the search for new stroke drugs is a major example of failed translation. More than 1000 candidates that seemed promising in animal studies, failed eventually in the clinic. Although clinical trials have to adhere to strict guidelines, rules for preclinical studies are not so binding. It has become more and more clear recently that also in these, mostly animal, studies, high quality of the study is necessary. Experiments have to be designed properly, sufficient numbers of animals should be calculated to be able to make a correct statement and doing experiments at several centres (multicentre study) will all increase the quality of the research. This will not only lead to better translatability to the clinic but also reduces the total amount of animals needed, since 1 medium sized study immediately gives a clear result, instead of all small studies with conflicting findings. This reduction of the amount of animals addresses nicely the 3R's of animal experimentation: reduction, refinement, and replacement. In addition, multi-centre trials will enhance cooperations and increase again knowledge and technique transfer. 
Validating good disease targets early in the process of drug development, together with good preclinical study practice, will in the end speed up the process of finding new treatments and bringing them to the clinic. Clinicians are then involved in clinical trials. However, also early in the process they can provide their expertise for example on individual variations in diseases. This is important for finding the right specific treatment to really treat a patient and not the disease. Also, clinicians can be involved in getting possible preventive measures into the clinic. Ultimately patients will then benefit from new treatments, increasing their quality of life. In addition, society will benefit both from the new treatments and from possible preventive measures, reducing mortality and morbidity and thus decreasing the total burden of health care.

Taken together, the new approach of finding specific mechanistic targets for cardiovascular disease using high quality preclinical research will lead to faster development of new treatments that can be taken into clinic. Patients will benefit from personalised medicine increasing their quality of life. In the same time, different research fields and industry will work together to entangle piece by piece the difficult puzzle of the diseasome.

\section{References}

1. Barabási, A.-L., Gulbahce, N. \& Loscalzo, J. Network medicine: a network-based approach to human disease. Nat. Rev. Genet. 12, 56-68 (2011). 


\section{Appendices}

\section{Dankwoord}

List of publications

About the author 


\section{Dankwoord}

Toen ik vier jaar geleden de stap van de kliniek naar basale wetenschap maakte, had ik nog geen idee wat het doen van een PhD inhield. Gedurende de jaren is me duidelijk geworden dat planning, creativiteit en een flinke portie doorzettingsvermogen onontbeerlijk zijn. Maar een promotieonderzoek en het schrijven van een proefschrift doe je niet alleen. Bij deze een woord van dank aan iedereen die een bijdrage heeft geleverd.

Allereerst Harald, mijn promotor; jij hebt me de kans gegeven om de overstap van de kliniek naar het onderzoek te zetten. Door jouw hulp heb ik me kunnen ontplooien van arts tot wetenschapper. Daarnaast hebben onze discussies, je schrijfvaardigheden en het kunnen bezoeken van congressen en cursussen mijn enthousiasme voor NOX en de wetenschap vergroot. Dit alles werd aangevuld door Harry, mijn tweede promotor, die meer op de achtergrond een adviserende rol heeft gehad.

Ben, als mijn co-promotor en hoofd van het in vivo lab was jouw hulp in het bedenken, opzetten en uitvoeren van de dierstudies en het analyseren van de resultaten ervan onmisbaar.

Agnieszka, Helma, Jacques en Peter: zonder jullie expertise in de in vivo experimenten en jullie enorme inzet was het onmogelijk geweest in de afgelopen korte periode van vier jaar alle experimenten uit te voeren en was dit proefschrift er nu niet geweest. Ook de expertise en inzet van Ger, Gregorio, Jet en Peter voor het non-in-vivo werk was onmisbaar. Een speciaal dankwoord ook voor de mensen van het lab van Christoph in Würzburg die eveneens een groot deel van de data gegenereerd hebben.

Dan natuurlijk mijn andere directe collega's in Maastricht: Kirstin, Rob, Kim en Sebastian, die mij met name in de eerste helft van het traject hebben bijgestaan met raad en daad. In de tweede helft van het traject kwamen daar nog Tessa en Thao-Vi bij. 
Uiteraard ook een woord van dank aan alle andere collega's van de afdeling Farmacologie voor de prettige jaren van samenwerking en de gezellige gesprekken bij de koffieautomaat.

Tijdens het promotietraject was natuurlijk ook 'het leven buiten de universiteit' een belangrijke factor om deze PhD tot een goed einde te brengen.

Pap en mam, jullie hebben de basis gelegd voor mijn wetenschappelijke carrière door mij de kans te geven te gaan studeren. Daarnaast hebben jullie mij altijd gesteund en gemotiveerd om dat te doen wat ik wilde en zo mijn dromen waar te maken.

Sylvia, mijn zus, is altijd mijn grote voorbeeld geweest in wier voetsporen ik ben getreden door geneeskunde te gaan studeren. Ook na mijn overstap na de wetenschap kon ik rekenen op jouw steun. Samen ook met Rob, mijn zwager, hebben we menig medisch en wetenschappelijk getint gesprek gevoerd aan tafel.

Lisa en Sanne, mijn twee petekindjes, jullie vrolijke gezichtjes en altijd aanwezige lach vormden een welkome afwisseling met de drukte en experimenten op het werk. Als laatste wil ik nog Wim bedanken, mijn partner. Alhoewel je alleen de laatste anderhalf jaar van mijn $\mathrm{PhD}$ hebt meegemaakt heb je juist in die periode een essentiële bijdrage geleverd. Je stond altijd voor me klaar, was het niet voor technische hulp met Photoshop of een eigenwijs Word-document, dan wel met een ontspannen weekend weg van het werk en de stress.

Rest mij niets meer dan twee woorden aan jullie allemaal en iedereen die ik vergeten ben bij naam te noemen: ontzettend bedankt! 


\section{List of publications}

Kleikers, P. W. M., Wingler, K., Hermans, J.J.R., Diebold, I., Altenhöfer, S., Radermacher, K.A., Janssen, B., Görlach, A., Schmidt, H.H.H.W. NADPH oxidases as a source of oxidative stress and molecular target in ischemia/reperfusion injury. J. Mol. Med. 90, 1391-1406 (2012).

van der Sluijs-Bens, J. P. F. \& Kleikers, P. W. M. [A premature with nail defects]. Ned Tijdschr Geneeskd 154, A205 (2010).

van der Sterren, S., Kleikers, P., Zimmermann, L. J. I. \& Villamor, E. Vasoactivity of the gasotransmitters hydrogen sulfide and carbon monoxide in the chicken ductus arteriosus. Am. J. Physiol. Regul. Integr. Comp. Physiol. 301, R1186-98 (2011).

Radermacher, K. A., Wingler, K., Kleikers, P.W.M., Altenhöfer, S., Hermans, J.J.R., Kleinschnitz, C., Schmidt, H.H.H.W., The 1027th target candidate in stroke: Will NADPH oxidase hold up? Exp Transl Stroke Med 4, 11 (2012).

Altenhofer, S., Kleikers, P.W.M., Radermacher, K.A., Scheurer, P., Hermans, J.J.R., Schiffers, P., Ho, H., Wingler, K., Schmidt, H.H.H.W., The NOX toolbox: validating the role of NADPH oxidases in physiology and disease. Cell Mol Life Sci 69, 2327-2343 (2012).

Wingler, K., Altenhoefer, S.A., Kleikers, P.W.M., Radermacher, K.A., Kleinschnitz, C., Schmidt, H.H.H.W., VAS2870 is a pan-NADPH oxidase inhibitor. Cell Mol Life Sci 69, 3159-3160 (2012).

Radermacher, K. A., Wingler, K., Langhauser, F., Altenhöfer, S., Kleikers, P., Hermans, J.J.R., Hrabe De Angelis, M., Kleinschnitz, C., Schmidt, H.H.H.W., Neuroprotection after stroke by targeting NOX4 as a source of oxidative stress. Antioxid Redox Signal 18, 1418-1427 (2013).

Altenhöfer, S., Radermacher, K. A., Kleikers, P., Wingler, K. \& Schmidt, H. H. Evolution of NADPH oxidase inhibitors: Selectivity and mechanisms for target engagement. Antioxid Redox Signal (2014). doi:10.1089/ars.2013.5814 


\section{About the author}

Pamela Kleikers was born on September 21, 1985, in Heerlen, the Netherlands. After spending her childhood in Waubach, she graduated from College Rolduc in Kerkrade in 2003 with a Natural Sciences profile (Natuur en Techniek Profiel) rounded off with Latin and Music. She then enrolled in Maastricht University's Medicine School, where in her final year she made a first and stimulating contact with research as part of the WESP (wetenschapsparticipatie) programme. In the laboratories of the Department of Paediatrics, Pamela investigated the vascular reactivity of the chicken ductus arteriosus and presented here thesis at the Maastricht Medical Student Research Conference at 2009. After graduating as a Medical Doctor (basisarts) in July 2009, she worked for one year at the Atrium Medisch Centrum in Heerlen as a resident at the paediatric ward before making the transition to basic medical research. For this she returned to Maastricht, now to the Department of Pharmacology to start her PhD in Prof. Harald Schmidt's Vascular Drug Discovery Group. During her PhD trajectory she presented her work as poster at several national and international conferences and gave invited oral presentations at the Society for Free Radical Research International in London in 2012 and the 2014 Gordon Research Conference on NADPH oxidases. In 2014 she also received the first price for her oral presentation at the PhysPharm Benelux Meeting in Maastricht and a Young Investigator Award from the Society for Free Radical Research Europe. In the first year of her PhD she successfully followed the Summer School on Cardiovascular Sciences 'From basic mechanisms to clinical application' organised by the Council on Basic Cardiovascular Science of the European Society of Cardiology. In the following years she also completed all three PhD courses organised by the Dutch Heart Foundation on 'Cardiac function', 'Vascular biology' and 'Thrombosis' and several PhD Summer Courses organised by the Cardiovascular Research Institute Maastricht. Next to her research activities, Pamela was involved as a tutor in the PGO programme of the first year medical students and as a Medical Student Examiner for both practical (stationstoets) and written (persoonlijk formularia) tests. She also supervised Bachelor and one Master intern students from the Biomedical Sciences track. After completing her PhD, Pamela's desire is to stay in academia and combine research with (medical) teaching and possibly some clinical work. 